\title{
IN SITU ANALYSIS OF THE MU- OPIOID RECEPTOR SPLICE VARIANTS IN ADULT RAT BRAIN
}

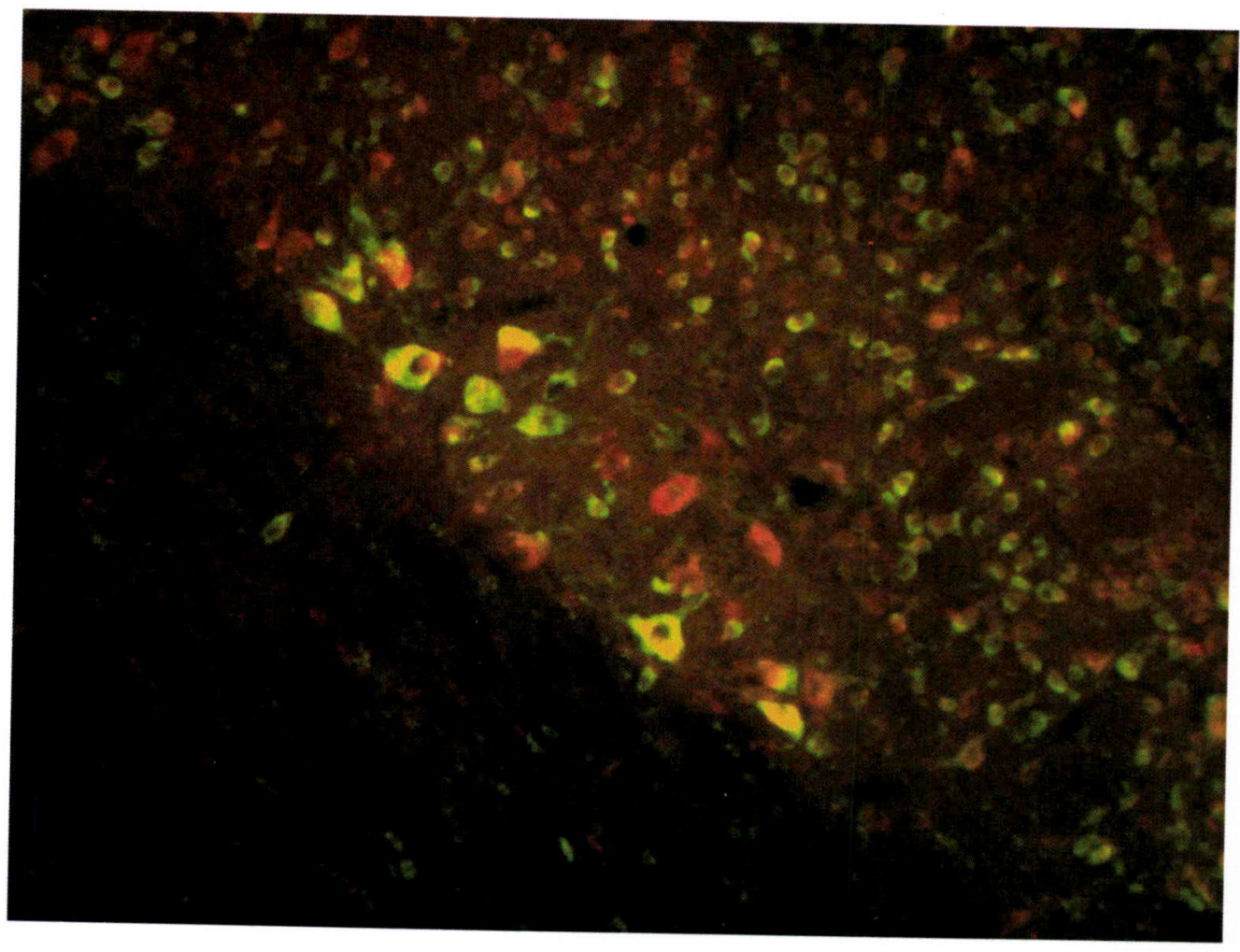

\section{Kylie M. Price}

Submitted in partial fulfilment of the requirements for the degree of Master of Science in Cell and Molecular Bioscience

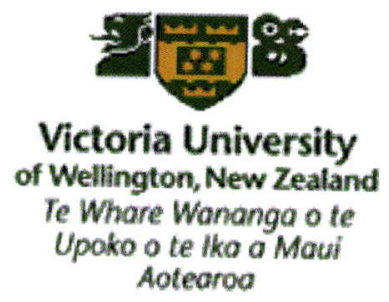

2003 


\begin{abstract}
The original intention of this study was to exploit the specificity of circularisable ligation probes (CLiPs) in a unique approach of in situ genotyping the mu-opioid receptor (MOR) splice variants. CLiPs were designed to target a PCR generated MOR-1 template in vivo. The ligation results were consistent with circularised CLiPs, however due to the inherent limitations of this method the more conventional technique of fluorescent in situ hybridisation (FISH) was substituted for CLiPs to analyse to distribution of MOR splice variants in rat brain.
\end{abstract}

Utilising FISH, the aim was to produce RNA probes (riboprobes) approximately the same size as the target specific region of CLiPs ( $60-70 \mathrm{nt})$ to analyse the distribution patterns of MOR splice variants in rat brain.

Five short (70-222 nt) riboprobes were generated to exons 1, 3, 4 and 9, and the 5' $\mathrm{UTR}+$ exon 1 of the Rattus norvegicus MOR gene (Oprm) to be utilised in FISH. The exon 1, 4 and 5' UTR + exon 1 riboprobes were shown to localise to MOR mRNA in brain structures previously reported to express MORs. These riboprobes also localised to mRNA within the Purkinje cells of the adult rat cerebellum, where it is generally accepted that only DOR is expressed in the rat cerebellum. MOR mRNA was visualised in many structures in the rat brain, including the dendate gyrus, inferior olive and spinal trigeminal nucleus. Riboprobes generated to the 5' UTR + exon 1 and exon 4 showed differential distribution patterns, the functional significance of this discovery is unknown, however these results implicate a role for FISH in tracking the distribution patterns of untranslated and translated mRNA.

The use of novel new short riboprobes represents a technically difficult yet feasible technique for mapping MOR mRNA distribution in adult rat brain. 


\section{Contents}

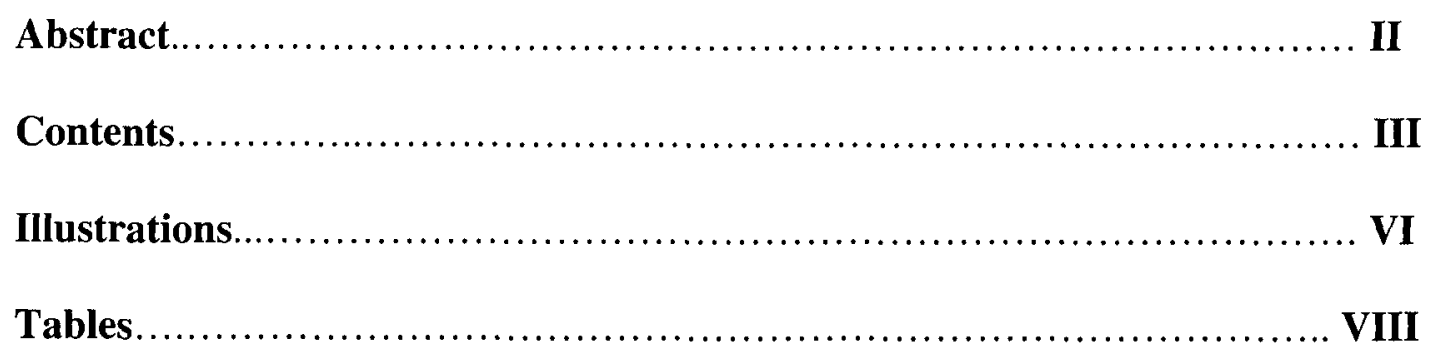

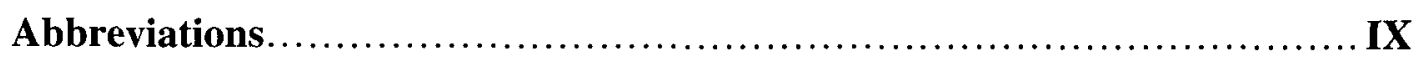

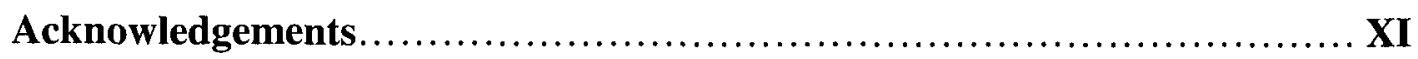

\section{Chapter 1. The Opioid System}

$1.0 \quad$ Introduction......................................................... 1

1.1 Opioid Receptors................................................... 3

1.2 Biological Effects of the Opioid Receptors........................ 7

1.2.1 Analgesia/Nociception......................................... 8

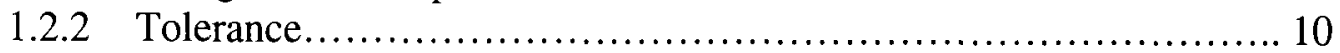

1.2.3 Respiration................................................... 11

1.3 Important Sites in the CNS Supporting the Opioid System............ 12

1.4 Mu-Opioid Receptor (MOR) Splice Variants......................... 15

1.4.1 Pharmacological Characterisation of MORs......................... 16

1.4.2 MOR Gene Structure and Splice Variants............................... 18

1.4.3 MOR Splice Variant Distribution.................................. 21

1.5 Techniques used to Map Opioid Receptor Distribution............... 22

1.5.1 Radiolabelled Binding Studies and Autoradiography................. 24

1.5.2 Immunohistochemistry............................................... 25

1.5.3 Reverse Transcriptase-Polymerase Chain Reaction (RT-PCR) ....... 27

1.5.4 Fluorescent in situ Hybridisation (FISH) .............................. 29

1.6 Aims of Thesis........................................................ 30

\section{Chapter 2. Analysis of MOR Splice Variants using} Circularisable Ligation Probes (CLiPs)

2.0 Introduction.......................................................... 32

2.1 Experimental Design............................................. 38

2.2 CLiP Design ..................................................... 41

2.3 Single-stranded CLiP Synthesis..................................... 43

2.3.1 Primer Extension................................................. 43

2.3.2 Affinity Capture ................................................. 45

2.4 Ligation of CLiPs................................................ 46

2.5 Results and Discussion............................................ 46 


\section{Chapter 3. Analysis of MOR Spice Variant Distribution Using Fluorescent in situ Hybridisation (FISH)}

3.0 Introduction................................................. 58

3.1 Fluorescent in situ Hybridisation................................... 59

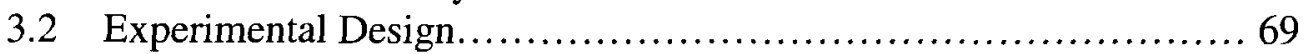

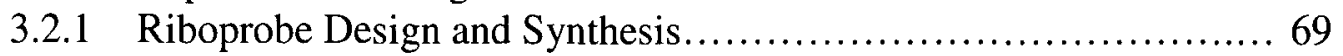

3.3 Riboprobe Purification........................................ 78

3.4 Results and Discussion............................................. 79

3.4.1 Riboprobe Synthesis............................................. 79

3.4.2 Analysis of Synthesised Riboprobes........................... 82

3.4.3 Interpreting FISH Results................................... 85

3.4.4 Image Adjustment............................................... 89

3.4.5 Exon 3 riboprobe............................................. 90

3.4.6 Exon 4 riboprobe................................................ 93

3.4.7 Exon 9 riboprobe................................................ 98

3.4.8 Hae III generated exon 1 riboprobe.............................. 101

3.4.9 Pvu II generated 5' UTR + exon 1 riboprobe...................... 103

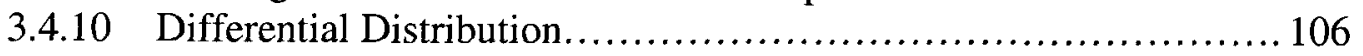

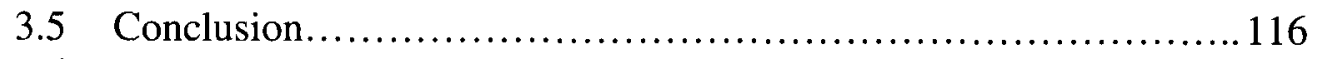

3.6 Future Work ................................................. 117

\section{Chapter 4. Experimental Procedures}

4.0 Primer synthesis.................................................. 119

4.1 Phosphorylation of primers................................... 120

4.2 CLiP synthesis.............................................. 121

4.2.1 Restriction digestion of $\mathrm{pBSSK}^{-}$containing the 5' UTR and exon 1 of Oprm............................................... 121

4.2.2 PCR synthesis of CLiP template................................ 121

4.2.3 PCR synthesis of CLiPs.................................... 122

4.3 PCR product analysis by submarine agarose gel electrophoresis.... 122

4.4 Purification of PCR products..................................... 123

4.4.1 Agarose gel extraction......................................... 123

4.4.2 PCR purification kit............................................ 123

4.5 Single-stranded CLiP synthesis................................. 124

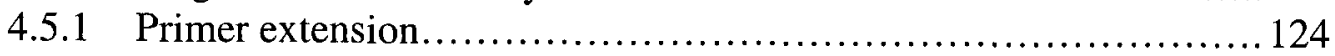

4.5.2 Affinity capture................................................. 125

4.6 PCR amplification of target for CLiP ligation....................... 125

4.7 CLiP ligation using Thermus thermophilus (Tth) ligase............. 126

4.8 Analysis of labelled PCR products and CLiPs using the ABI PRISM ${ }^{\circledR}$

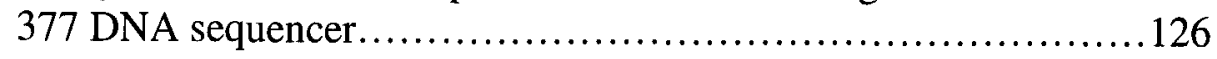

4.9 DNA quantification............................................. 127

4.10 Bacterial cultures............................................. 127

4.10.1 Maintenance of bacterial cultures............................. 127 
4.10.2 Purification of plasmids............................................. 128

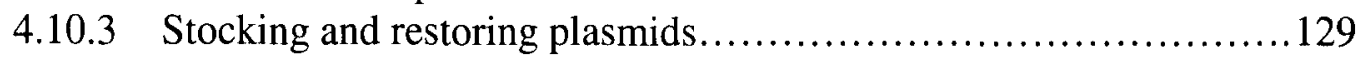

4.11 Sephadex G-25 spin columns..................................... 129

4.12 Generation of riboprobe template using overlapping oligonucleotides (oligos) ....................................................... 130

4.12.1 Generation of ds DNA template for exon 4 and 9 riboprobe synthesis using overlapping oligos................................. 130

4.12.2 Generation of exon 4 and 9 riboprobe templates using second round PCR fill-in.................................................... 130

4.12.3 Generation of ds DNA template for riboprobe synthesis using the Klenow fragment................................................ 131

4.12.4 Polishing of 3'-ends using T4 DNA polymerase......................131

4.13 Generation of riboprobe template using PCR and restriction digestion.

4.13.1 Restriction digestion of the mammalian GATEWAY pDEST ${ }^{\mathrm{TM}} 12.2$

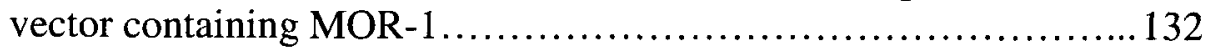

4.13.2 PCR synthesis of exon 3 riboprobe template.........................132

4.13.3 PCR synthesis of the MOR 287 fragment...........................133

4.13.4 Restriction digestion of MOR 287 fragment to generate the template for exon 1 and $P v u I I$ riboprobe synthesis...........................133

4.14 Riboprobe synthesis via in vitro transcription using T7 RNA polymerase......................................................... 134

4.15 Desalting riboprobes and purifying small PCR products by ethanol precipitation....................................................... 135

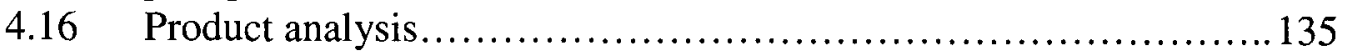

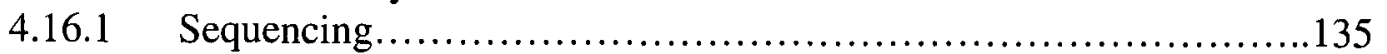

4.16.2 Size and quality analysis of fluorescently labelled riboprobes.........136

4.16.3 Product quantification using a SYBR Green II assay ..................136

4.17 Tissue preparation..................................................139

4.17.1 Perfusion protocol and tissue fixation............................... 139

4.17.2 Cutting and mounting sections.................................... 140

$4.18 \quad$ FISH.......................................................... 140

4.18.1 Prehybridisation................................................... 140

4.18.2 In situ hybridisation.................................................. 141

4.18.3 Posthybridisation................................................. 141

4.19 Analysis of FISH experiments........................................... 142

4.19.1 Fluorescent microscopy............................................... 142

4.19.2 FISH images.................................................. 142

Appendix A Size Standards and Markers....................................... 143

Appendix B Chromatogram of sequence MOR exon 3 riboprobe.................144

Appendix C Design of MOR primers for riboprobe synthesis..................... 146

Appendix D Staining of different structures in adult rat brain using riboprobes...148

Appendix E Raw data of signal and background intensities.....................150

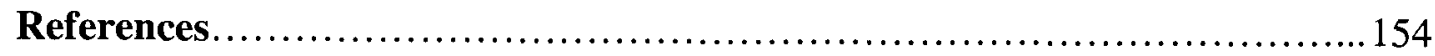




\section{Illustrations}

1.0 Schematic representation of opioid receptor structure, ligand binding sites and regulation sites.

1.1 An overview of the role endogenous opioids and their receptors play in nociception........................................................ 13

1.2 Schematic representation of the three major brain structures involved in the reward pathway................................................. 14

1.3 Schematic representation of MOR-1 gene (Oprm) and splice variants...... 20

2.0 Schematic representation of CLiP structure hybridised and ready for ligation....................................................... 32

2.1 Schematic representation of the primer structure designed for CLiP synthesis......................................................... 33

2.2 Schematic representation of ds CLiP template synthesis................. 34

2.3 Schematic representation of enzymatic CLiP synthesis and ss CLiP purification using affinity capture.

2.4 Flowchart depicting experimental design of labelled ss CLiPs and the eventual progression to FISH using small riboprobes.....................40

2.5 Analysis of CLiP target sequence to region of MOR-1 gene................ 42

2.6 Schematic representation of primer extension........................... 44

2.7 Synthesis of ds CLiP template............................................ 47

2.8 Synthesis of ss CLiPs using primer extension PCR .......................... 48

2.9 Optimising primer extension PCR ................................... 49

2.10 Labelled primer extension PCR, producing F-dUTP labelled ss CLiPs........ 51

2.11 Second round PCR producing labelled ds CLiPs..........................53

2.12 Eluate from affinity capture containing ss CLiPs......................... 55

2.13 Analysis of ligation reactions on the ABI PRISM ${ }^{\circledR} 377$ DNA Sequencer using GeneScan ${ }^{\circledR}$ Analysis Software version $3.7 \ldots \ldots \ldots \ldots \ldots \ldots \ldots \ldots \ldots \ldots . . \ldots 6$

3.0 Positive control experiment using $\mathrm{NaOH}$-treated tissue in a FISH............67 67

3.1 Schematic overview of exon 3 riboprobe synthesis........................ 71

3.2 Schematic overview of exon 4 riboprobe synthesis......................... 73

3.3 Schematic overview of exon 9 riboprobe synthesis...................... 75

3.4 Schematic overview of the 5' UTR + exon 1, and exon 1 riboprobe synthesis......................................................... 77

3.5 Synthesis of the T7-tailed exon 4 riboprobe template by PCR ............. 80

3.6 In vivo T7-RNA transcription of exon 4 riboprobes....................... 80

3.7 Synthesis of the MOR 287 fragment by PCR, including unlabelled exon 4 and exon 9 riboprobes.

3.8 Restriction digestion of the MOR $287 \mathrm{bp}$ fragment to generate the exon 1 and $P v u I I$ riboprobe templates............................... 81

3.9 Labelled riboprobes synthesised to exon 1 and the 5' UTR + exon 1........ 82

3.10 Size determination of a FITC-labelled exon 9 riboprobe................... 83

3.11 Top two BLAST search results using exon 4 sequence...................... 84

3.12 Saggital section 1.2 of adult rat brain near midline......................... 89

3.13 Image manipulations............................................... 90

3.14 CY3-labelled exon 3 riboprobes........................................ 91 
3.15 FITC-labelled exon 4 riboprobes........................................... 96

3.16 Exon 9 riboprobe localisation in adult rat brain........................... 89

3.17 FITC-labelled exon 1 riboprobes...................................... 102

3.18 FITC-labelled $P v u$ II generated riboprobes............................ 105

3.19a Differential staining of CY3-labelled exon 4 riboprobes and FITC-labelled riboprobes............................................. 107

3.19b Differential staining of CY3-labelled exon 4 riboprobes and FITC-labelled 287 fragment riboprobes............................... 108

3.20a FISH images of CY3-labelled exon 4 riboprobes and FITClabelled exon 1 and $P v u$ II generated riboprobes..........................111

3.20b Differential staining of CY3-labelled exon 4 riboprobes and FITClabelled MOR 287 fragment riboprobes in the inferior olive............... 112

3.21 Differential staining of CY3-labelled exon 4 riboprobes and FITClabelled exon 1 and MOR 287 fragment riboprobes in the inferior olive...115

$4.0 \mathrm{a}$ Plate reading from a SYBR Green II assay ............................137

4.0b Microtitre plate chart.............................................. 138

4.1 Standard curve from a SYBR Green II assay generated by diluting

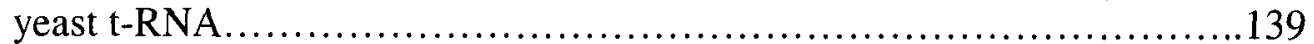

A1 $\quad 1 \mathrm{~Kb}^{+}$DNA ladder size standard ............................................. 143

A2 The ABI PRISM ${ }^{\circledR}$ GeneScan $^{\text {TM }}$-350 ROX size standard.................. 143

B1a Bascaller-377 version 3.7 analysis of ds DNA template for exon 3 riboprobe synthesis using the exon 3 forward primer for sequencing....... 144

B1b Comparison of exon 3 sequence generated using the exon 3 forward primer and the exon 3 reverse sequence.................................. 144

B2 Basecaller-377 version 3.7 analysis of ds DNA template for exon 3 riboprobe synthesis....................................................... 145

C1 Exon 3 riboprobe designed to MOR-1 mRNA............................. 146

C2 Overlapping oligonucleotides designed to exon 4 of MOR-1 mRNA..... 146

C3a MOR 287 fragment designed to MOR-1 mRNA.......................... 146

C3b Primer sequences used to generate the MOR 287 fragment.................. 147

C4 The 3' RACE sequence of MOR-1C mRNA used to design the exon 9 overlapping oligonucleotides........................................... 147

C5 Target region of CLiP designed to Mus musculus MOR-1 mRNA.......... 147

D1 FITC-labelled exon 4 riboprobes staining in the dendate gyrus.............. 148

D2 FITC-labelled $P v u$ II generated riboprobes staining the endopiriform nuclei................................................................. 148

D3 FITC-labelled $P v u$ II generated riboprobes staining the anterior and posterior interposed cerebellar nuclei...................................... 149

D4 Exon 9 riboprobes staining in the parvocellular lateral reticular nucleus... 149

E1 Pixel intensity data obtained from histogram analysis using Adobe Photoshop version 5.5 ..................................................... 151 


\section{Tables}

\section{Table Number}

\section{Page Number}

1.0 Mammalian endogenous opioid peptides and their precursor proteins........ 2

1.1 Structures in the brain involved in producing a physiological state via the opioid receptors....................................................... 8

1.2 Opioid receptor selective agonists and antagonists...................... 17

1.3 Comparison of MOR splice variant distribution in structures of the central nervous system..................................................... 21

3.0 A list of the main advantages and disadvantages associated with each

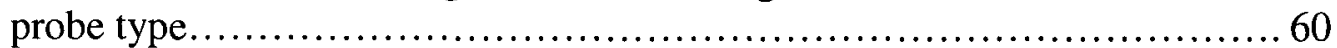

3.1 The critical parameters used to design PCR primers for riboprobe synthesis.. 69

3.2 Table of confidence for signal intensities............................... 88

4.0 Primer sequences used to generate CLiPs and MOR-1 target template.......119

4.1 Sequences used to generate riboprobes to specific exons of MOR...........120 


\section{Abbreviations}

\begin{tabular}{|c|c|}
\hline ASPCR & Allele specific polymerase chain reaction \\
\hline bp & Base pairs \\
\hline cAMP & Cyclic adenine monophosphate \\
\hline CLiP & Circularisable ligation probe \\
\hline CNS & Central nervous system \\
\hline cRNA & Copy ribonucleic acid \\
\hline CY3 & Cyanine-3 \\
\hline DADLE & {$\left[\mathrm{D}-\mathrm{Ala}^{2}, \mathrm{D}-\mathrm{Leu}^{5}\right]$ enkephalin } \\
\hline DAMGO & {$\left[\mathrm{D}-\mathrm{Ala}^{2}, \mathrm{~N}-\mathrm{methyl}-\mathrm{Phe}^{4}, \mathrm{Gly}^{5}-\mathrm{ol}\right]$ enkephalin } \\
\hline DAPI & 4',6'-Diamidine-2'-phenylindole dihydrochloride \\
\hline DEPC & Diethyl-pyro carbonate \\
\hline DIG & Digoxigenin \\
\hline DOR & Delta opioid receptor \\
\hline DPDPE & {$\left[D-P^{\frac{1}{2}, 5}\right]$ enkephalin } \\
\hline ds & Double stranded \\
\hline $\mathrm{EtBr}$ & Ethidium bromide \\
\hline FISH & Fluorescent in situ hybridisation \\
\hline F-12-dUTP (F-dUTP) & Fluorescein-12-2'deoxy-uradine-5' -triphosphate \\
\hline FITC & Fluorescein isothiocyaniate \\
\hline G-protein & Guanine nucleotide binding-protein \\
\hline GPCR & G-protein coupled receptor \\
\hline HRP & Horseradish peroxidase \\
\hline ICC & Immunocytochemistry \\
\hline IgG & Immunoglobulin \\
\hline IHC & Immunohistochemistry \\
\hline IO & Inferior olive \\
\hline ip & Intraperitoneal \\
\hline ISH & In situ hybridisation \\
\hline IS-RT-PCR & In situ-reverse transcriptase-polymerase chain reaction \\
\hline KOR & Kappa opioid receptor \\
\hline LCR & Ligase chain reaction \\
\hline LDR & Ligase detection reaction \\
\hline MCS & Multiple cloning site \\
\hline M6G & Morphine- $6 \beta$-glucuronide \\
\hline MOR & Mu opioid receptor \\
\hline $\mathrm{NAb}$ & Nucleus ambiguus \\
\hline NMDA & N-methyl D-aspartate \\
\hline $\mathrm{nt}$ & Nucleotide(s) \\
\hline NTPs & Nucleotide triphosphates \\
\hline Oligos & Oligonucleotides \\
\hline OR & Opioid receptor \\
\hline ORL-1 & Opioid receptor-like receptor \\
\hline Oprm & Mu opioid receptor gene \\
\hline PAG & Periaqueductal grey \\
\hline PAGE & Polyacrylamide gel electrophoresis \\
\hline PB & Parabrachial nucleus \\
\hline
\end{tabular}


PCR

PNS

POMC

RVM

SA-beads

SDS

SNP

SS

TB

TSA

Tth

UTP

UTR

VTA
Polymerase chain reaction

Peripheral nervous system

Pro-opiomelanocortin

Rostral ventromedial medulla

Streptavidin coated paramagnetic beads

Sodium dodecyl sulphate

Single nucleotide polymorphism

Single stranded

Transcription buffer

Tyramide signal amplification

Thermus thermophilus

Uridine triphosphate

Untranslated region

Ventral tegmental area 


\section{Acknowledgements}

Thanks to Darren Day for teaching me so many things about both life and science.

Thanks to Eli for the long and interesting conversations about politics and the corrupt world at large! You've been a good friend and have definitely tickled the grey matter over the past few years and it has been a lot of fun.

Danke to Lornie for always being there for me no matter what, you've been an inspiration to me for a long time and words could never express what a difference you have made to my life. I would like to thank Mum for being proud of me, and setting the standards for what women can achieve, you've helped me more than you may know.

Thanks to Uncle Doug and Aunty Gill for always being interested and supportive, I've appreciated all you've done for me over the years, from Germany and beyond.

Thanks to the connoisseur of life Charles for all your help, support, laughter and wine! You're a friend for life mate. Thanks also to Langers for just being you.

JU! What to say, I would never have finished without you. I think the sounding board may need a bit of polishing after the pounding it took for this!! Thank you for your patience and understanding (you are a wise one you know) and for always letting me be me!! The music, laughter and relaxing time we've had are in my memory banks forever, thanks Sam.

And last but by no means least, danke mein Schatz. Dams you are everything to me: best friend, biggest fan, source of laughter and the most supportive partner one could ever wish for. Thank you so much for believing in me even when the times got rough, you know when to push me and when to leave me be and that balance enabled me to be writing this page! Danke again, and into the future we've been dreaming of babe.

\section{Perseverance prevails when all else fails}




\section{Chapter 1}

\section{The Opioid System}

\subsection{Introduction}

The opioid receptors are the primary targets of opiates and endogenous opioid peptides. The opium poppy papaver somniferum has been used extensively throughout history to relieve pain. In 1803, a crystalline sample of the principle alkaloid, morphine, was isolated from papaver somniferum. Later this was shown to be almost entirely responsible for the analgesic activity of unprocessed opium (Corbett et al.).

A key side effect of morphine use is respiratory depression, in 1942 the first opiate antagonist, nalorphine, was produced. This antagonist was capable of reversing the respiratory depression caused by morphine administration (Weiljlard and Erikson 1942). Even though nalorphine could counter the actions of morphine, it could still be used as an analgesic agent, meaning that this drug was a mixed agonist-antagonist (Brownstein 1993). An explanation of how mixed agonists and antagonists might work, led to the general consensus of multiple opioid receptors. Radiolabelled drugs were used to try and characterise the multiple opioid receptors (Goldstein et al. 1971). These binding experiments showed that in the central nervous system there were stereospecific opiate binding sites (Pert and Snyder 1973, Simon et al. 1973, Terenius 1973). 
The first endogenous opioid ligands to be successfully isolated were from porcine brain. These were the two pentapeptides Tyr-Gly-Gly-Phe-Leu (Leu-enkephalin) and Tyr-Gly-Gly-Phe-Met (Met-enkephalin) (Hughes et al. 1975). Later it was observed that the N-terminus of another molecule, $\beta$-endorphin, shared the same Met-enkephalin sequence and also had a high affinity for brain opioid receptors (van Ree et al. 1976). In 1981 the dynorphins were discovered due to their structural similarity to enkephalins (Goldstein et al. 1981). Three different precursor proteins give rise to the enkephalins, endorphins and dynorphins. These precursors are known as pro-enkephalin (Noda et al. 1982), pro-opiomelanocortin (POMC) (Nakanishi et al. 1979) and pro-dynorphin (Kakidani et al. 1982), respectively. Two new classes of endogenous opioid peptides called endomorphins (Zadina et al. 1997) and nociceptin/orphanin FQ (Butour et al. 1997) have been recently discovered. These peptides are generated from proteolysis of the precursor proteins proendomorphin and pro-nociceptin/OFQ, respectively (Table 1.0).

\begin{tabular}{|c|c|}
\hline Precursor Protein & Endogenous Opioid Peptide \\
\hline Pro-opiomelanocortin & $\beta$-endorphin \\
\hline Pro-enkephalin & $\begin{array}{l}\text { [Met]enkephalin } \\
\text { [Leu]enkephalin }\end{array}$ \\
\hline Pro-dynorphin & $\begin{array}{l}\text { Dynorphin A } \\
\text { Dynorphin A(1-8) } \\
\text { Dynorphin B } \\
\alpha \text {-neoendorphin } \\
\beta \text {-neoendorphin }\end{array}$ \\
\hline Pro-nociceptin/OFQ & Nociceptin \\
\hline Pro-endomorphin & $\begin{array}{l}\text { Endomorphin-1 } \\
\text { Endomorphin-2 }\end{array}$ \\
\hline
\end{tabular}

Table 1.0 Mammalian endogenous opioid peptides and their precursor proteins. 
Opioid receptors and endogenous opioid peptides are distributed widely throughout the mammalian central, peripheral and autonomic nervous systems, as well as in most endocrine tissues and organs (Kornblum et al. 1987, Mansour et al. 1987, Smith et al. 1993). They have a wide range of pharmacological and physiological effects including: tolerance and dependence; cardiovascular responses; respiration and thermoregulation; gastrointestinal, renal, and hepatic function; mental illness and mood; learning, memory and reward; seizures and other neurological disorders (Vaccarino et al. 1999).

\subsection{Opioid Receptors}

Three classes of opioid receptors have been identified. The first convincing evidence of multiple opioid receptors came from studies using congeners of morphine to produce three different syndromes in the nondependent chronic spinal dog (Martin et al. 1976). These syndromes were attributed to the presence of three distinct receptors: the morphine $(\mu)$ opioid receptor (MOR), ketocyclazocine $(\kappa)$ opioid receptor $(\mathrm{KOR})$ and the $\mathrm{SKF}-10,047(\sigma)$ opioid receptor. The sigma receptor is generally considered not to be an opioid receptor.

After the enkephalins were discovered, research began into which receptors these opioid peptides interact with. One of the studies involved inducing contractions of guinea pig ileum using electrical currents (Lord et al. 1977). It was noted that morphine was more effective than enkephalins in inhibiting contractions in the ileum, but surprisingly that enkephalins were more active in inhibiting contractions in the vas deferens. Furthermore, naloxone (a potent opiate antagonist) had little effect on the action of enkephalins at the vas deferens. It was based on these findings 
that a fourth receptor called the delta $(\delta)$ opioid receptor (DOR) was postulated to be present in the vas deferens (Lord et al. 1977).

It is currently accepted that the three main classes of opioid receptors ( $\mu, \delta$ and $\kappa$ ) can be further subdivided. Based on clinical observations and pharmacological studies opioid receptor splice variants have been identified for MOR (Wolozin and Pasternak 1981, Pasternak and Wood 1986), DOR (Jiang et al. 1991, Mattia et al. 1991b, Mattia et al. 1991a, Sofuoglu et al. 1991) and KOR (Zukin et al. 1988, Cheng et al. 1992) mRNAs. There is also research to support the existence of two other opioid receptors namely the zeta receptor (Zagon et al. 1991) and more recently the opioid receptor-like receptor (ORL-1) (Butour et al. 1997).

The three most well defined receptors are MOR, KOR and DOR. Using expression cloning in mammalian cells the first cDNA encoding murine DOR (mDOR) was isolated in 1992 (Evans et al. 1992, Kieffer et al. 1992). The MOR and KOR cDNAs were identified by homology cloning (Gaveriaux et al. 1995, Kieffer 1995). Hydrophobic analyses of mDOR cDNA revealed that this receptor had seven putative transmembrane helices which is a trait of the guanine nucleotide binding (G)-protein-coupled receptor family (Kenakin 1996).

Overall, MOR, DOR and KOR are $60 \%$ identical to each other. The transmembrane regions share the greatest homology with $73-76 \%$ identity. The extracellular and intracellular regions differ significantly, sharing only $34-40 \%$ identity (Satoh and Minami 1995). The most divergent of these regions are the second and third extracellular loops, as well as the $\mathrm{N}$ - and C-termini (Jordan et al. 2000) (Figure 1.0). 
Selective binding of DAMGO,

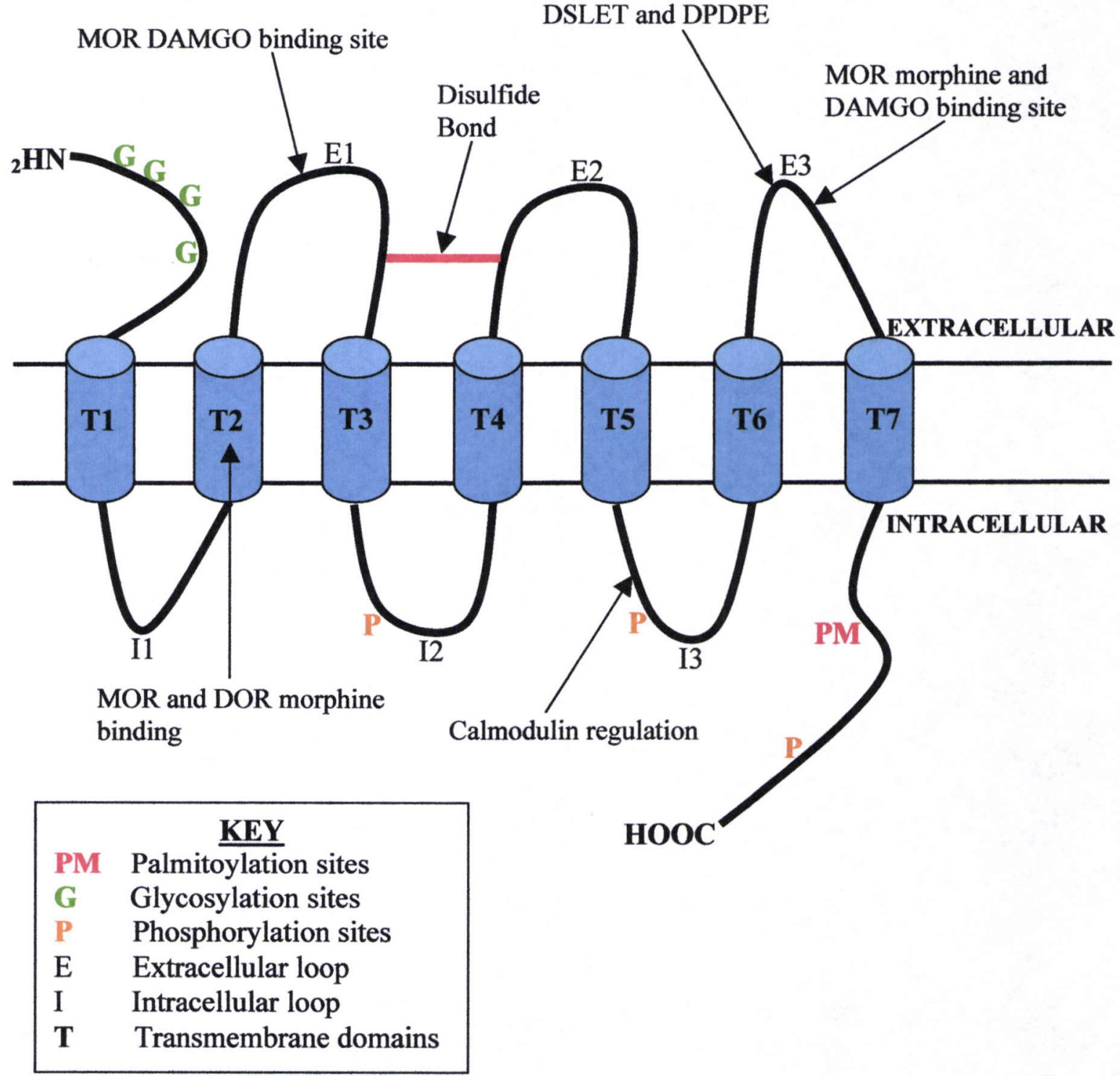

Figure 1.0 Schematic representation of opioid receptor structure, ligand binding sites and regulation sites, adapted from Kivell. 2003.

The opioid receptors (ORs) are accepted as being G-protein coupled receptors, belonging to the rhodopsin receptor superfamily. The opioid receptors are not coupled directly with stimulatory G-proteins, $\mathrm{G}_{\mathrm{s}}$ or $\mathrm{G}_{\mathrm{q}}$ (Corbett et al.), but as members of the rhodopsin superfamily they are coupled to the inhibitory G-protein $\mathrm{G} \alpha_{\mathrm{i}}$. This protein inhibits the activation of adenylate cyclase therefore blocking the 
production of the secondary messenger cyclic AMP (cAMP). Stimulation of the opioid receptors also activates inwardly rectifying $\mathrm{K}^{+}$channels and decreases the conductance of voltage operated $\mathrm{Ca}^{2+}$ channels (North et al. 1987).

Cellular responses to opiate stimulation are mediated by receptor phosphorylation, either by endogenous specific G-protein coupled receptor kinases (GRKs) or nonspecific protein kinases (protein kinase $\mathrm{A}$ or $\mathrm{C}$, and $\mathrm{Ca} 2+/$ calmodulin-dependent protein kinase II). Currently there are six known isoenzymes of the GRKs (GRK16) that transfer phosphate groups onto the serine or threonine residues of the Cterminus of the ORs.

Receptor desensitisation is defined as "a rapid loss of receptor function upon sustained exposure to an agonist" (Higgins 1998), and it has been postulated that modulation of the GRKs may be one of the molecular mechanisms involved in receptor desensitisation (Mestek et al. 1995). Other molecular mechanisms have also been proposed to explain receptor desensitisation, for example opioid receptor dimerisation. Studies have shown that DOR exists as a dimer when expressed in heterologous cells (Cvejic and Devi 1997), and that KOR also exists as a dimer that is stable in sodium dodecyl sulphate (SDS) (Jordan et al. 2000). It has also been shown that kappa receptors can heterodimerise with delta, but not mu receptors (Jordan et al. 2000). This may represent a novel regulatory mechanism that could either restrict or enhance phosphorylation and desensitization of the $G$ proteincoupled opioid receptors (Pfeiffer et al. 2002). 


\subsection{Biological Effects of the Opioid Receptors}

ORs are widely distributed throughout the mammalian central and peripheral nervous systems (CNS and PNS respectively), and have long been implicated in the control of various physiological functions (Atweh and Kuhar 1983). Although the elucidation of OR function is far from complete, they are known to play a role in many biological activities such as: long term potentiation, locomotion, pain perception (nociception), respiration, immunity, stress response and many other biological behaviours (Vaccarino et al. 1999).

The transient appearance of ORs and their endogenous peptides (Bayon et al. 1979) in certain brain and spinal regions during development has led to the hypothesis that endogenous opioids may serve a functional role in development that is quite distinct from their role in the adult.

ORs appear early in the ontogeny of the CNS with each receptor type having unique developmental expression patterns. Radioligand binding assays, autoradiography and in situ hybridisation (ISH) experiments have shown that MOR and KOR expression is first detectable within the embryonic rat brain by the twelfth to fourteenth day (E12-E14) of gestation (Attali and Vogel 1990, Georges et al. 1998, Tong et al. 2000). DOR is almost undetectable in embryonic rat brain, but has been shown to appear at, or shortly after birth (Georges et al. 1998).

MOR densities are particularly high throughout development in regions of the brain involved in respiratory function, such as the nucleus tractus solitarius (NTS) and the nucleus ambiguous (Xia and Haddad 1991). MOR expression levels continue to rise 
until two weeks after birth, when they reach their peak and decrease to adult levels (Tong et al. 2000). The exact role of the opioid receptors during development is not known, however from recent OR knockout studies it has been noted that these receptors play vital roles in haematopoiesis, reproductive physiology (Tian et al. 1997) and modulating locomotion (Kieffer and Gaveriaux-Ruff 2002). Table 1.1 shows brain structures involved in specific physiological effects, characterised by the ORs present.

\begin{tabular}{|r|l|}
\hline $\begin{array}{l}\text { Pharmacological or } \\
\text { Physiological Function }\end{array}$ & \multicolumn{1}{|c|}{ Region of Brain } \\
\hline Nociception/Analgesia & $\begin{array}{l}\text { Pontine parabrachial nucleus (PB), Periaqueductal grey } \\
\text { matter (PAG), the rostral ventromedial medulla, the } \\
\text { dorsal horn of the spinal cord and trigeminal ganglion } \\
\text { sensory neurons, spinal trigeminal nucleus, cuneate, } \\
\text { gracile nuclei, and thalamus regions }\end{array}$ \\
\hline $\begin{array}{r}\text { Motivation/Reward/ } \\
\text { Reinforcement }\end{array}$ & $\begin{array}{l}\text { Ventral tegmental area (VTA) } \\
\text { Nucleus accumbens (NAc) }\end{array}$ \\
\hline Respiration/Hypoxia/ & $\begin{array}{l}\text { Preoptic area } \\
\text { Hypothermia } \\
\text { Morphine-controlled respiration: Nucleus of solitary } \\
\text { tract, nucleus ambiguous, parabrachial nucleus }\end{array}$ \\
\hline Addiction & Nucleus accumbens \\
\hline Sedation & Mesencephalic reticular formation \\
\hline Nausea/Vomiting & Neurons of the postrema \\
\hline
\end{tabular}

Table 1.1 Structures in the brain involved in producing a physiological state via the opioid receptors.

\subsubsection{Analgesia/Nociception}

One important function of the opioid system is the role it plays in modulating nociception. Exogenous $\mu, \kappa$ and $\delta$ opioid agonists have been used throughout history to produce analgesia. The neurotransmitter substance $\mathrm{P}(\mathrm{SP})$ and endogenous opioid ligands are intimately involved in the regulation of acute and chronic pain transmission (Go and Yaksh 1987, Kar and Quirion 1995, Minami et al. 1995). Double in situ hybridisation studies and northern blot analyses have been used to show that the precursor protein to SP and the mRNA of MORs and KORs, co- 
localise at the superficial laminae of the dorsal horn and in dorsal root ganglia of rat spinal cord (Kar and Quirion 1995, Minami et al. 1995). The superficial lamina of the dorsal horn is an important site of functional integration and transmission of nociceptive inputs. It has been strongly suggested from pharmacological data that SP released in the dorsal horn plays an important role in antinociception by regulating opioid analgesic activity and maintaining opioid responsiveness (Kream et al. 1993, Foran et al. 2000).

The dorsal horn receives nociceptive input from the PNS and these signals are then sent to the pontine parabrachial nucleus $(\mathrm{PB})$ in the brain. The $\mathrm{PB}$ is a major recipient of fibres from nociceptive spinal and trigeminal dorsal horn neurons. The ventrolateral periaqueductal gray (PAG) plays an important role in antinociception. Endogenous opioids or excitatory amino acids such as $\mathrm{N}$-methyl D-aspartate (NMDA) can activate their respective receptors to dampen pain transmission in the PAG (Commons et al. 1999). The PAG also contains a high density of MORs, which are involved in antinociception.

The analysis of MOR-, KOR- and DOR-deficient mice has demonstrated the importance of the opioid system in pain perception and has given positive results regarding targets for the treatment of pain. In models of acute pain the opioid receptor or peptide knockout mice generally showed an increased sensitivity to acute noxious stimuli, these results are in keeping with the pharmacology of the opioid system (Kieffer and Gaveriaux-Ruff 2002). 


\subsubsection{Tolerance}

Tolerance to a drug is defined as the failure of a steady dose of the drug over time to sustain the desired pharmacological effect (i.e. the need to increase the drug dosage to maintain the original pharmacological effect). Tolerance can occur with a wide variety of drugs, including opioids. There are three main regulatory mechanisms thought to contribute to opioid tolerance. These are receptor desensitisation (Borgland 2001, Alvarez et al. 2002), receptor internalisation (Gastard 2000, Eisinger et al. 2002), and alterations in second messenger systems (Sharma et al. 1975, Ferguson 2001).

Morphine is well known to produce tolerance, even though morphine administration does not promote rapid desensitisation or receptor endocytosis when studied in heterologous expression systems (Keith et al. 1996, Sternini et al. 1996). Comparatively, the endogenous opioids (e.g. endomorphins and etorphine) all seem to cause both rapid desensitisation and receptor internalisation. It has been reported that the ability of various agonists to differentially regulate MOR internalisation and desensitisation, is based on their capacity to promote $G$ protein-coupled receptor kinase (GRK)-dependent MOR phosphorylation (Zhang et al. 1998b).

$\beta$-arrestins come from a family of regulatory scaffold proteins that function in signal transduction at phosphorylated $G$ protein-coupled receptors, including ORs. As $\beta$ arrestins form large complexes on the intracellular C-termini of the receptors, they prevent G-proteins and other downstream activators from interacting with these receptors, resulting in desensitisation (Ferguson 2001). These receptor complexes are endocytosed at clathrin-coated pits and gradually recycled. Even though both 
etorphine and morphine activate MORs, only etorphine elicits robust MOR phosphorylation, followed by plasma membrane translocation of $\beta$-arrestin and receptor internalisation. In contrast, morphine is unable to elicit MOR phosphorylation or to stimulate $\beta$-arrestin translocation (Fan et al. 2003).

Therefore, the differing ability of agonists to produce receptor desensitisation and internalisation may provide the molecular basis underlying differences between the analgesic properties of various opioid agonists. Further investigations into this area may allow for the discovery of opioid analgesics that do not cause tolerance or crosstolerance. This would be of great benefit to patients with acute persistent pain e.g. terminal cancer patients.

\subsubsection{Respiration}

One major side effect of exogenously administered opioid analgesics is respiratory depression. Respiratory depression is produced by opiate agonists of the $\mu, \kappa$ and $\delta$ ORs and is mainly due to the inhibition of bulbar respiratory neurons (Morin-Surun et al. 1984a). Activation of MOR and DOR decreases the firing of these neurons with a subsequent reduction in respiratory rhythm and tidal volume (Morin-Surun et al. 1984b). One mechanism proposed to cause respiratory depression has been outlined in a recent study. Immunohistochemical techniques have revealed the presence of MOR splice variants in the pre-Bötzinger complex, a region of the brain hypothesised to generate respiratory rhythm (Gray et al. 1999). Agonists of MOR and KOR have also been shown to cause a reduction in final motor outputs, by both pre- and postsynaptic inhibition of the medullary inspiratory neural networks (Takeda et al. 2001). Research in this field is currently focusing on the addition of 
new reagents (e.g. NMDA receptor antagonists) to diminish the side effect of respiratory depression that is encountered using opioid analgesics such as anaesthetic.

When an organism is faced with low levels of oxygen, hypoxia is the result. Hypoxia causes hypothermia in most mammals due to excessive heat loss. The opioid system is believed to be involved in adaptive responses that occur during hypoxia to facilitate survival. Many experiments have been able to show a significant increase of endogenous opioids in animal models of hypoxia (GonzalezGuerrero et al. 1993, Kraczkowski and Semczuk 2000). Activation of DORs has been demonstrated to protect cortical neurons from glutamate-induced injury during hypoxia. Because glutamate mediates the hypoxic injury in neurons, it has been suggested that DORs play a crucial role in neuroprotection during hypoxia (Zhang et al. 2002).

\subsection{Important Sites in the CNS Supporting the Opioid System}

The opioid receptors are spread throughout the entire CNS and many studies have been undertaken to elucidate the precise location of the MOR, DOR and KOR splice variants. Techniques such as immunohistochemistry, autoradiography, microinjection mapping studies and in situ hybridisation studies have been applied to discrete brain regions, in order to identify sites and structures involved in the biological effects of the ORs. These studies have revealed a number of sites that appear to be very important for OR actions, such as analgesia, motivation and reward. 


\section{Periaqueductal Gray and Rostral Ventromedial Medulla}

The two most studied areas of the brain in relation to the opioid system and analgesia are the periaqueductal gray (PAG) and the rostral ventromedial medulla (RVM) (Mayer and Price 1976, Yaksh 1988). The PAG and RVM are the two main central nociceptive modulatory networks, they receive input from the dorsal horn nociceptive processes of the spinal cord and each area contains within it the neural machinery to induce analgesia (Stein 1999). Only the RVM send substantial descending projections to the dorsal horn. The PAG does not project to the dorsal horn, instead its sends large projections to the RVM. Figure 1.1 represents a model of descending nociceptive pathways.

\section{Descending Pain Control Pathways}

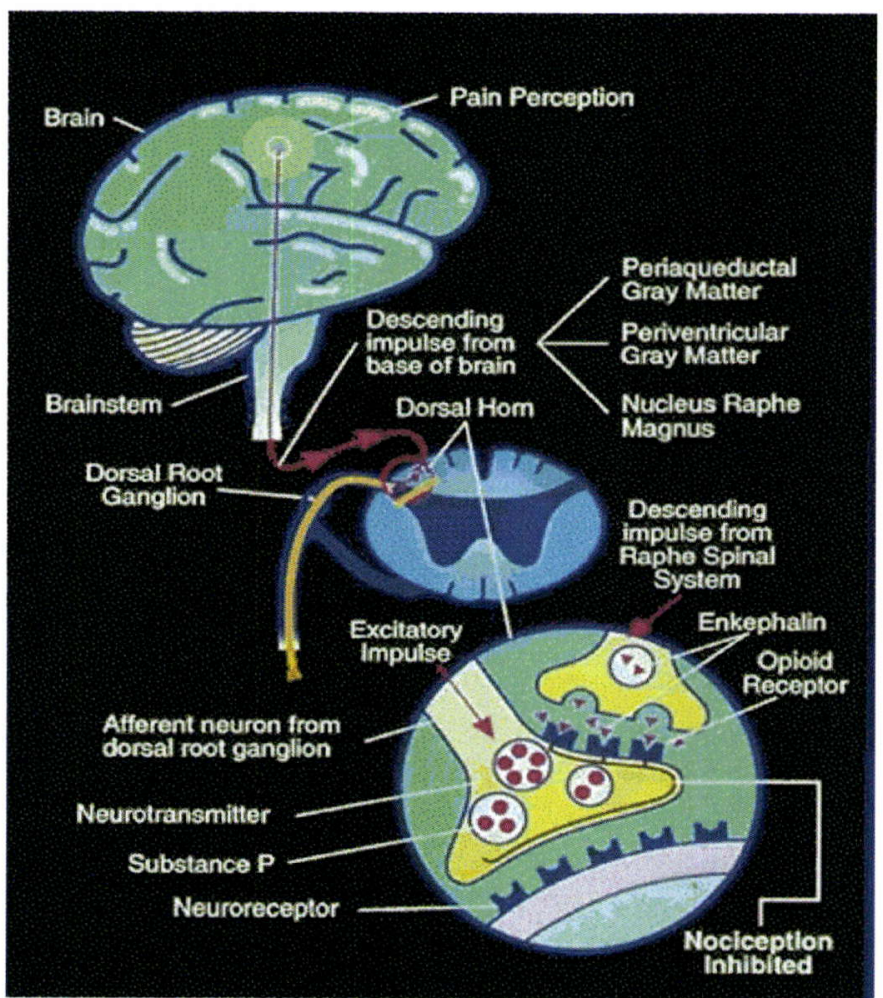

Figure 1.1 An overview of the role endogenous opioids and their receptors play in nociception. The periaqueductal grey (PAG) and the nucleus raphe magnus [located within the rostral ventromedial medulla (RVM)] are depicted above. Figure 1.1 shows the antinociceptive pathway going from an afferent dorsal root ganglion neuron to the PAG and RVM of the brain. This image is courtesy of the Roxane Pain Institute used with permission http://pain.roxane.com/sitemap.html 


\section{Ventral Tegmental Area, Nucleus Accumbens and Prefrontal Cortex}

The ventral tegmental area (VTA), the nucleus accumbens and the prefrontal cortex are three major structures thought to play an important role in reinforcement and reward pathways (Koob and Bloom 1988). The VTA is connected to both the nucleus accumbens and the prefrontal cortex and it sends information to these structures (Figure 1.2) via its neurons. When neurons of the VTA are activated by a stimulus, endogenous opioids are released in the nucleus accumbens and in the prefrontal cortex (You et al. 1998, Zangen and Shalev 2003). It has been shown that drug abuse causes a down-regulation of the inhibitory G-proteins $\left(G_{i} \alpha 1\right.$ and $\left.G_{i} \alpha 2\right)$ in the nucleus accumbens. McLeman et al found that these G-proteins were reduced by $32-49 \%$ in heroin and methamphetamine users (McLeman et al. 2000). This selective response suggests that the nucleus accumbens may also have a special role in drug reinforcement and drug dependent states such as tolerance, which are likely to be related to the inhibition of $\mathrm{G}_{\mathrm{i}} \alpha$-linked receptor activity.

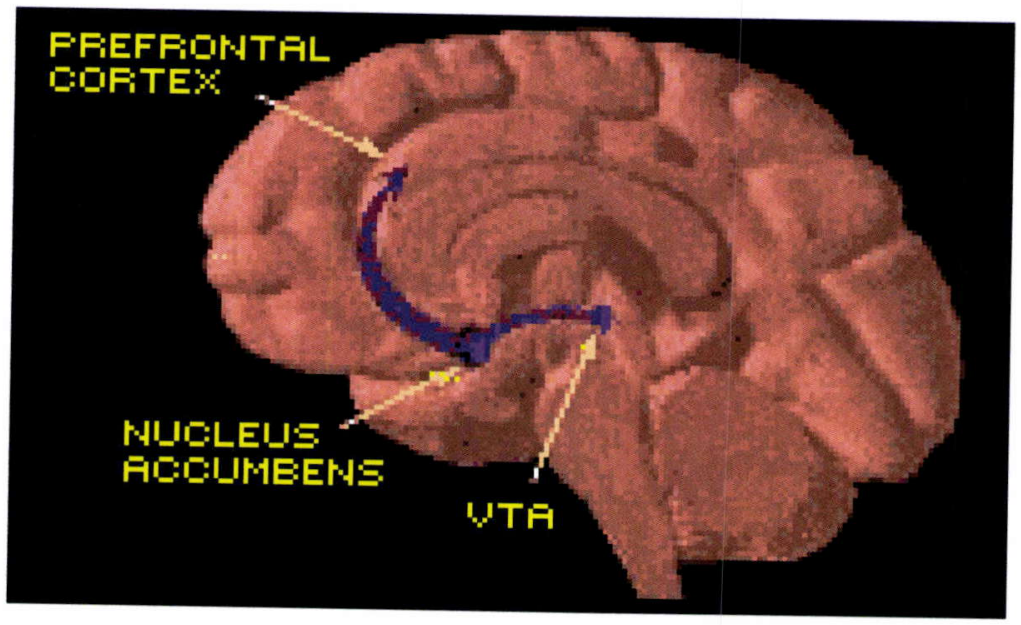

Figure 1.2 Schematic representation of the three major brain structures involved in the reward pathway. The image shows a human brain that has been cut in the sagittal plan. Image courtesy of NIDA (http://www.nida.nih.gov/). 


\section{Cerebellum and Inferior Olivary Nucleus (Inferior Olive)}

The cerebellum is an integral part of the body's motor system because it regulates posture, gait and voluntary movements. It coordinates the action of a series of participating muscle groups in order to achieve a specific movement by sending excitatory inputs to the cerebral cortex via the thalamus. The basal ganglia works opposite to the cerebellum, sending inhibitory signals via the thalamus to the cerebral cortex (Hendelman 1994). All climbing fibres, which are motor afferents coming from the spinal cord, must cross the midline of the inferior olive, before travelling onto the cerebellum. The inferior olive is located lateral to the pyramidal tract on the medulla and receives sensory and motor information, via numerous tracts (e.g. PAG), about the movements the cerebellum has just performed. This information is processed by the nuclear olive and sent back to the cerebellum via the inferior cerebellar peduncle to inform the cerebellum how well the movement was performed (Hendelman 1994).

\subsection{Mu-Opioid Receptors (MOR) Splice Variants}

Traditional opioid analgesics, such as morphine, exert their effects via MORs. The discovery of MOR splice variants has enabled scientists to better understand the complex pharmacology of mu opioid analgesics. The functional significance of the unique regional distribution and pharmacology of the MOR splice variants is not yet known. However, the differential expression of MOR splice variants in specific tissues could be a mechanism of control, which may explain such events as incomplete cross-tolerance. 


\subsubsection{Pharmacological characterisation of MORs}

In 1964 several types of ORs were postulated after the observation that nalorphine did not antagonise different narcotic analgesics to the same degree (Cox and Weinstock 1964, Veatch et al, 1964).

Radiolabelled drugs were initially used to characterise these ORs (Goldstein et al. 1971). The first definitive studies to prove the existence of the postulated three types of ORs ( $\mu, \kappa$ and $\delta$ ) were based on the pharmacological actions of various opioid alkaloids and their derivatives (Martin et al. 1976). Each type of OR exhibits distinct yet overlapping ligand-binding properties and functional characteristics, for example MOR and DOR bind enkephalins and endorphins, while KOR binds dynorphins. The invention of opioid receptor-specific antagonists has aided the pharmacological understanding of each receptor type.

DAMGO (Tyr-D-Ala-Gly-MePhe-NH( $\left(\mathrm{CH}_{2}\right) 2 \mathrm{OH}$ ) (Kosterlitz and Paterson 1981) is a highly selective MOR ligand and studies have shown that it is the first extracellular loop of the MOR that is critical for DAMGO binding (Surratt et al. 1994, Onogi et al. 1995) (Figure 1.0). MOR and DOR only differ in this region by seven amino acid residues, yet DOR has a very low affinity for DAMGO $\left(\mathrm{K}_{\mathrm{i}}=>1000 \mathrm{nM}\right)$ (Satoh and Minami 1995).

The DOR selective ligand is DPDPE ([D-Pen2, D-Pen5]-enkephalin) (Mosberg et al. 1983 ) and the KOR selective ligand is U-50,488 (trans-3,4-dichloro-N-methyl-N-[2- 
(1-pyrrolidinyl)cyclohexyl]-benzeneacetamide (von Voigtlander et al. 1983). These agonists have been used along with OR antagonists (e.g. naloxone and nalorphine) to characterise the pharmacological properties of the ORs (Table 1.2). There is a degree of cross-reactivity between the agonists and antagonists for all the ORs, due to their high levels of homology.

\begin{tabular}{|c|c|c|c|c|}
\hline $\begin{array}{l}\text { Receptor } \\
\text { Type }\end{array}$ & MOR & DOR & KOR & ORL-1 \\
\hline $\begin{array}{l}\text { Selective } \\
\text { Agonists }\end{array}$ & $\begin{array}{l}\text { *DAMGO } \\
\text { *Endomorphin-1 } \\
\text { *Endomorphin-2 } \\
\text { *DADLE }\end{array}$ & $\begin{array}{l}* \text { *DPDPE } \\
*\left[\text { D-Ala }{ }^{2}\right]- \\
\text { deltorphin I } \\
*[\text { D-Ala }]- \\
\text { deltorphin II } \\
\text { *DADLE }\end{array}$ & $\begin{array}{l}* \text { *U-50 488 } \\
* \text { U-69 } 593 \\
\text { *Enadoline } \\
\text { *Dynorphin A } \\
(1-17)\end{array}$ & $\begin{array}{l}\text { *Nociceptin/Orphanin } \\
\text { FQ }\end{array}$ \\
\hline $\begin{array}{l}\text { Selective } \\
\text { Antagonists }\end{array}$ & $\begin{array}{l}\text { *Naloxone } \\
\text { *CTAP }\end{array}$ & $\begin{array}{l}\text { *Naltrindole } \\
{ }_{\text {*TIPP }-\psi}\end{array}$ & $\begin{array}{l}\text { *Norbinaltorphimine } \\
* \text { Naloxone }\end{array}$ & *JTC-801 \\
\hline
\end{tabular}

Table 1.2 Opioid receptor selective agonists and antagonists. Ligands with the highest selectivity for each receptor type are represented in bold type.

The possibility of multiple MORs was considered after radioligand binding assays (using $\left[{ }^{3} \mathrm{H}\right]$-labelled $\mu$-ligands) displayed biphasic binding characteristics (Wolozin and Pasternak 1981).

Furthermore, several observations showed that analogues of morphine with

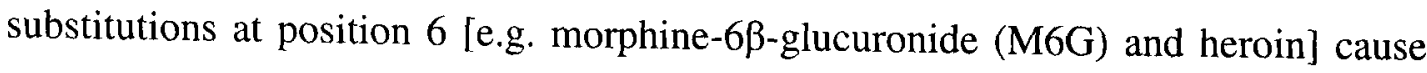
potent antinociception in the $\mu$-receptor deficient CXBX strain of mice, which is 
insensitive to morphine. It was determined that the 6-substituted morphine analogues do not act through DOR or KOR, as $\kappa$ - or $\delta$-receptor selective antagonists do not block the antinociception (Rossi et al. 1996, Brown et al. 1997).

After the discovery of the mu opioid receptor gene (Oprm) (Chen et al. 1993, Wang et al. 1993), MOR-1 knockout mice were engineered by disrupting the Oprm gene in exon 1. Interestingly, M6G and heroin were still able to produce antinociception in these mice, where morphine had no effect. It was therefore concluded that the antinociceptive actions of $\mathrm{M} 6 \mathrm{G}$ and heroin must be mediated through a MOR, which was produced by alternative transcription of the Oprm, and differed from the MOR-1 gene product in the region of exon 1.

\subsubsection{MOR Gene Structure and Splice Variants}

Early clinical and subsequent molecular studies supported the existence of two MOR isoforms with differing pharmacological properties (Pasternak and Snyder 1975, Pasternak et al. 1980a, b). Shortly after the cloning of MOR-1 cDNA (Chen et al. 1993, Wang et al. 1993), two groups reported that the 3 '-end of exon 3 is involved in alternative splicing. The structure of the Oprm gene was originally determined by analysis of a genomic P1 phage clone and the putative promoter region was revealed using RNase protection.

Because the ORs are members of the G-protein coupled receptor (GPCR) family, the discovery that their genes were alternatively spliced did not come as a surprise, as 
alternative splicing has been observed for a number of other GPCRs, including prostaglandin EP3 (Namba et al. 1993) and dopamine $D_{2}$ (Guiramand et al. 1995).

PCR cloning and 5' rapid amplification of cDNA ends (5' RACE) were used to identify previously unknown exons upstream from exon 1 (Pan et al. 2001). There are currently 14 known exons of the Oprm gene, spanning $250 \mathrm{~kb}$ and generating 15 MOR splice variants (Figure 1.3). Exons 11 and 12 are spliced onto the 5'-terminus of Oprm (Pan et al. 2001), and are therefore upstream of exon 1. Exons 13 and 14 are situated between exons 1 and 2 and all splice variants contain exons 2 and 3 (Figure 1.3). MOR-1 - MOR-1F all contain exon 1, MOR-1A lacks exon 4 and MOR-1B is alternatively spliced to exon 5, instead of exon 4 as with MOR-1 (Bare et al. 1994, Zimprich et al. 1995).

In 2001 Pan et al. identified a previously unknown promoter $10 \mathrm{~kb}$ upstream from exon 1 that controls exon 11 , meaning the three splice variants MOR-1H, MOR-1I and MOR-1 J contain two potential translational start points (Figure 1.3). The exon 1 start point generates three proteins that are indistinguishable from the original MOR1 protein.

Therefore MOR- 1 can be generated from four different alternative splicing events of Oprm, under the control of two distinct promoters (Pan et al. 2001). The splice variants under the control of the exon 11 promoter (MOR-1G - MOR-1N) are all truncated proteins, but still show unique regional distribution throughout a rat brain. 
A.

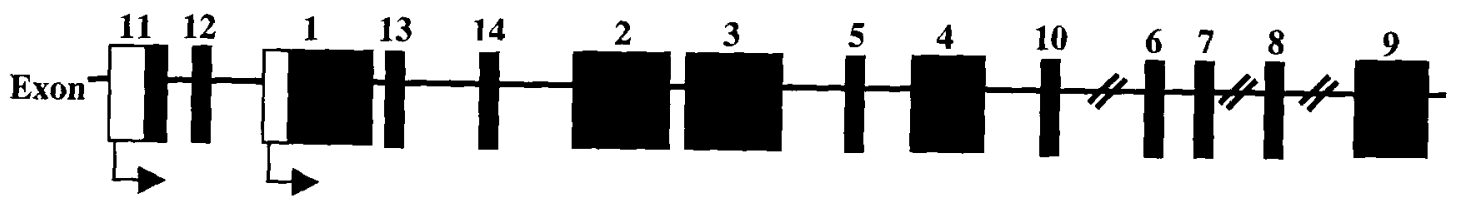

B.

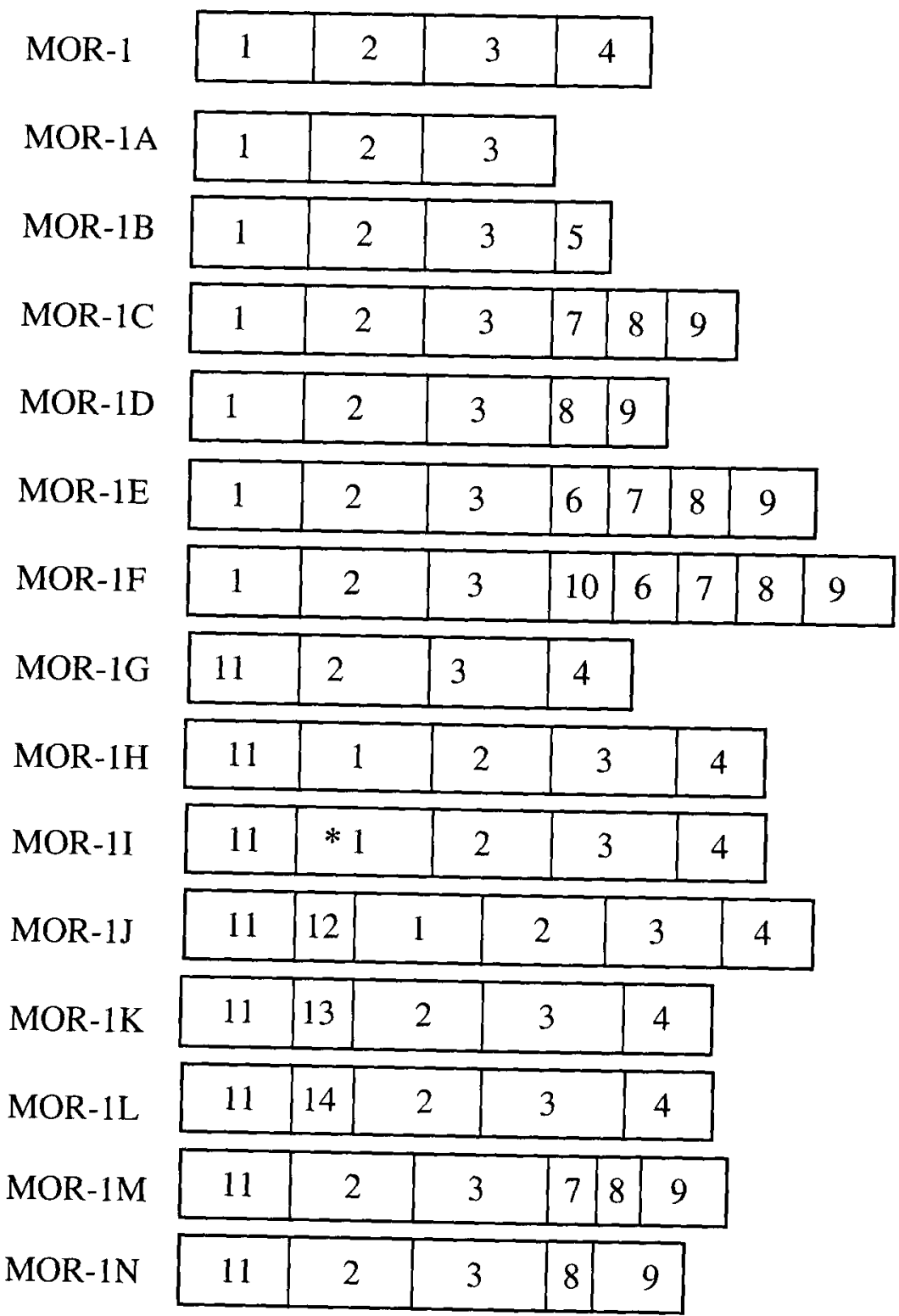

Figure 1.3 Schematic representation of MOR-1 gene (Oprm) and splice variants. A. Coding exons are represented by black boxes and 5' flanking regions by blank boxes. The arrows depict physically distinct promoters of Oprm. The exons are numbered in the order they were discovered (adapted from (Pan et al. 2001). B. Depicts the exon composition of all 15 MOR-1 splice variants, the * in MOR-1I depicts the transcription start site, as this splice variant contains both promoters but is regulated by the promoter in exon 1 . 


\subsubsection{MOR Splice Variant Distribution}

MOR splice variants are localised both pre- and post-synaptically but are expressed almost exclusively on GABAergic interneurons (Ding et al. 1996) and are widely distributed throughout the mammalian adult central and peripheral nervous systems (CNS and PNS, respectively). Table 1.3 demonstrates the vast distribution of MOR splice variants. This table was compiled from multiple sources and many different localisation techniques, therefore is best used as a general guide to distribution patterns.

\begin{tabular}{|c|c|}
\hline $\begin{array}{l}\text { Splice } \\
\text { Variant }\end{array}$ & Region of Brain \\
\hline MOR-1 & $\begin{array}{l}\text { Perikarya of mitral cells and distinct juxtaglomerular cells. Sensory trigeminal complex, } \\
\text { median raphe nuclei and periaqueductal grey matter (PAG) (Schulz et al. 1998). Brain stem, } \\
\text { hypothalamus and thalamus (Pan et al. 2001). Presubiculum and parasubiculum, amygdaloid } \\
\text { nuclei, hippocampal formation, thalamic nuclei, and nucleus ambiguous (Abbadie et al. } \\
\text { 2000a). Subcallosal streak in the striatum, medial habenular nucleus, medial terminal } \\
\text { nucleus of the accessory optic tract, interpeduncular nucleus, parabrachial nuclei, locus } \\
\text { coeruleus, nucleus of the solitary tract, and laminae I and II of the medullary and spinal } \\
\text { dorsal horns (Ding et al. 1996). }\end{array}$ \\
\hline MOR-1B & $\begin{array}{l}\text { Subcallosal streak in the striatum, medial habenular nucleus, medial terminal nucleus of the } \\
\text { accessory optic tract, interpeduncular nucleus, median raphe nucleus, parabrachial nuclei, } \\
\text { locus coeruleus, ambiguous nucleus, nucleus of the solitary tract, and laminae I and II of the } \\
\text { medullary and spinal dorsal horns (Ding et al. 1996). External plexiform layer of the main } \\
\text { olfactory bulb localized to a dense plexus of dendrites mostly originating from mitral cells } \\
\text { and extending into the glomerular layer (Schulz et al. 1998). }\end{array}$ \\
\hline MOR-1C & $\begin{array}{l}\text { Lateral parabrachial nucleus, the periaqueductal grey, and laminae I-II of the spinal } \\
\text { trigeminal nuclei and the spinal cord. Lateral septum, and most hypothalamic nuclei such as } \\
\text { the median eminence, periventricular, suprachiasmatic, supraoptic, arcuate, paraventricular, } \\
\text { ventromedial, and dorsomedial nuclei (Abbadie et al. 2000a). }\end{array}$ \\
\hline MOR-1D & $\begin{array}{l}\text { Dendate gyrus and in the mossy fibres of the hippocampal formation, the nucleus of the } \\
\text { solitary tract and the area postrema, the inferior olivary nucleus, the nucleus ambiguous, the } \\
\text { spinal trigeminal nucleus and the spinal cord (Abbadie et al. 2000b). }\end{array}$ \\
\hline MOR-1E & PAG, brain stem, hypothalamus and cortex (Pan et al. 1999). \\
\hline MOR-1G & PAG, spinal cord, hypothalamus and cortex (Pan et al. 2001). \\
\hline MOR-1H & Thalamus and striatum (Pan et al. 2001). \\
\hline MOR-1I & Hypothalamus and spinal cord (Pan et al. 2001). \\
\hline MOR-1J & Thalamus, spinal cord, PAG and hypothalamus (Pan et al. 2001). \\
\hline MOR-1K & a cord, hypothalamus (Pan et al. 2001). \\
\hline MOR-1L & Spinal cord (Pan et al. 200I). \\
\hline MOR-1M & Spinal cord (Pan et al. 2001). \\
\hline MOR-1N & Spinal cord (Pan et al. 2001). \\
\hline
\end{tabular}

Table 1.3 Comparison of MOR splice variant distribution in structures of the central nervous system. These results have all been obtained using different localisation techniques. NB. Currently there is no data about the regional distribution of MOR-1A and MOR-1F splice variants. 
${ }^{32} \mathrm{P}$-labelled probes were generated by PCR to specific MOR exons and used in northern blot analysis on total mRNA from mouse brain. This technique determined the lengths of the transcripts encoding each new variant discovered (Pan et al. 1999, Pan et al. 2001). Rudimentary experiments were initially used to examine the general distribution of MOR splice variant mRNA. Experiments such as reversetranscription polymerase chain reaction (RT-PCR), were used on total RNA isolated from different regions of mouse brain (Pan et al. 1999, Pan et al. 2001). Using primers designed from the sequences of MOR exons 3, 4, 9 and 11, it was possible to obtain data relating to the general distribution of 13 MOR splice variants (Pan et al. 1999, Pan et al. 2001).

Radioligand binding studies (Mansour et al. 1987), immunohistochemistry (Mansour et al. 1995) and in situ hybridisation (ISH) (Mansour et al. 1994) techniques have been used to analyse MOR splice variant protein and mRNA distribution throughout the CNS and peripheral tissues. Comparing results obtained from these various techniques, it has been noted that there is a good correlation between protein and mRNA expression patterns, with only slight differences being observed. These differences can be accounted for by the inherent advantages and disadvantages of each technique, which are discussed in more detail in section 1.5.

\subsection{Techniques used to Map Opioid Receptor Distribution}

A vast array of techniques has been used to detect and characterise the ORs. Radiolabelled drugs were initially used to identify these hypothesised receptors and after the discovery of the endogenous opioids, radiolabelled binding studies were 
used to elucidate the pharmacology and molecular biology of the ORs. Localisation studies began shortly after the discovery of the ORs.

Northern blot analysis is commonly used to identify the presence of ORs and their expression patterns in specific brain structures. This is one method that does not involve ISH, but has been frequently used to determine MOR splice variant distribution. Here total RNA is extracted from a specified brain region and a complementary labelled probe is allowed to hybridise. Sizes and relative abundance of mRNA transcripts can be determined by northern blot analysis. However the relative abundance of a specific RNA does not normally represent the actual amount of mRNA transcripts, as the probe is unable to distinguish between transcripts destined for degradation or translation. Another disadvantage of northern blot analysis is that specific morphological localisation data cannot be obtained, as total RNA is extracted from brain regions and analysed, without the benefits of ISH.

ISH methods were developed due to the need to identify nucleic acid sequences within morphologically preserved cells, chromosomes or tissue sections. ISH techniques have been significantly refined since their introduction in the late $1960 \mathrm{~s}$ (Gall and Pardue 1969b, John et al. 1969). They now give investigators the ability to define which cells within a mixed population express the mRNA or contain the gene of interest. ISH allows the relation of gene expression to the topology of the sample, (e.g. expression patterns of MORs within brain tissue). The requirements for ISH are adequate probe labelling and specificity, with ISH techniques mainly varying by the type of label used (e.g. isotopic, non-isotopic fluorescent or non-fluorescent). ISH is currently the technique of choice in a wide range of molecular biological fields, e.g. 
cell-cell interactions, RNA/DNA viruses or cellular localisations of endogenous receptors, due to the resolution of signal provided by the new probe labelling molecules and the topological data one can receive using this technique.

\subsubsection{Radiolabelled Binding Studies and Autoradiography}

In vitro studies used radioactive isotopes, such as ${ }^{3} \mathrm{H}$, to label OR-selective ligands in order to confirm the existence of multiple ORs. 'This method allowed the distribution patterns of ORs in brain sections to be examined (Mansour et al. 1987), with radiolabelled ligand procedures producing more pharmacologically relevant data than ISH. Radiolabelled binding methods are not an in situ hybridisation (ISH) technique, as the ligands do not complementarily hybridise to nucleic acid, rather this technique exploits binding affinities of ligands to identify receptor distribution. Therefore some topological data can be obtained, however there is an increased probability of ligands binding non-specifically to other receptors, especially the other opioid receptor subtypes, as they have such a high percentage of sequence identity.

Autoradiographic ISH is a technique whereby a DNA or cRNA probe is used for hybridisation and is labelled with a radioactive isotope. An advantage of autoradiography is the possibility of quantitation by grain counting (Lewis et al. 1989). Radioactive labels have significant disadvantages, such as high cost, safety considerations, poor probe stability (e.g. ${ }^{32} \mathrm{P}$ has a very short half-life of 14 days), long exposures being required (e.g. longer than 1 month), poor resolution due to grain size and no double labelling being possible. However radiolabelled probes are still frequently used because of their high sensitivity, which is necessary for detecting low copy number mRNA transcripts. Autoradiography has largely been superseded 
by safer non-isotopic labelling methods, that use haptens such as biotin and digoxigenin (DIG) to label DNA or RNA probes for ISH experiments.

A new technique called 'functional autoradiography' has been successfully combined with other in vitro assays, like receptor autoradiography and in situ hybridization histochemistry to localise ligand-binding sites. It is a novel method unifying the advantages of receptor-autoradiography and $\left[{ }^{35} \mathrm{~S}\right]$ GTPgammaS binding. Agonist binding and subsequent G-protein coupled receptor activation are detected simultaneously giving the exact location of the activated receptor (Sovago et al. 2001). This method still has the disadvantages associated with radioactivity.

\subsubsection{Immunohistochemistry}

Immunohistochemistry (IHC) and immunocytochemistry (ICC) both utilise antibodies to localise proteins. The only difference being that IHC localises proteins in whole tissue sections (e.g. MOR distribution in specific brain structures), whereas ICC pinpoints intracellular proteins (e.g. MORs at axon terminals) typically in cultured cells.

The primary antibody can be directly labelled with an enzyme (such as horseradish peroxidase, HRP), an isotope or a fluorophore (such as fluorescein isothiocyaniate, FITC) or remain unlabelled, with detection via a labelled secondary antibody. If a secondary antibody is used it must be generated against the immunoglobulins (IgGs) of the primary antibody source. There are several different methods used to detect bound antibodies, but the method of choice is mainly determined by whether the antibody is labelled with an enzyme or a fluorophore. Labelled primary antibodies 
enable direct signal detection, whereas unlabelled primary antibodies require labelled secondary antibodies and a signal amplification cascade. Therefore indirect signal detection methods can create problems in the interpretation of results, as non-specific signals are amplified and may give a high ratio of false positives.

Immunohistochemistry has, to date, provided the majority of data about the regional distribution of the ORs and their splice variants. Many studies have examined the differential distribution of MOR splice variants using antibodies directed to unique amino acid sequences within each splice variant, typically the $\mathrm{N}$ - or C-terminus (Mansour et al. 1995, Abbadie et al. 2000a). Advantages of immunohistochemistry include the wide availability of reagents and equipment required (e.g. light microscope) and the comparative rapidity of the procedure.

The MOR splice variant protein distribution results obtained using immunohistochemistry are directly comparable to results obtained using autoradiography, RT-PCR, ISH and FISH. One consideration is that the MOR splice variant specific antibodies also bind degraded and inactive receptors, thus giving an indication only of the total amount of MOR splice variant protein present. This technique gives no indications about the specific activity of the receptors.

There are many disadvantages associated with immunohistochemistry. There is no standard scoring system with which one can quantitatively compare results, even when standard quantitative microscopy methods are used. There is no uniformly accepted intensity threshold, so a scale involving the intensity of immunoreactivity (e.g. high, moderate, low or negative) is typically used. The generation and 
purification of either monoclonal or polyclonal antibodies to the specific target protein is time consuming and can be very expensive.

A major disadvantage is the specificity and cross-reactivity of the antisera used, especially when trying to identify regional distribution patterns of MOR splice variants, which have high levels of similarity at the amino acid level. Specific antisera have been produced to MOR splice variants, however time consuming assays (e.g. antisense treatment) must be performed in order to confirm their specificity and selectivity, before they can be used to produce raw data.

\subsubsection{Reverse Transcriptase-Polymerase Chain Reaction (RT-PCR)}

PCR was formulated to mimic the features of naturally occurring DNA replication (Mullis and Faloona 1987) such as the use of DNA polymerases that synthesise new complementary strands under the direction of two deoxyoligonucleotide primers. These primers largely govern the specificity for PCR and their sequences flank the DNA segment of interest. PCR involves the use of a thermostable DNA polymerase in a thermal cycle of heating and cooling, which controls DNA strand dissociation, hybridisation and replication.

Sensitive methods are required for the detection and analysis of low copy number mRNA transcripts, therefore amplification of mRNA is sometimes necessary, which is where the PCR methodology has been adapted to incorporate RNA. As RNA itself cannot act as a template in PCR it is first reverse transcribed to cDNA, using a 
reverse transcriptase, and then PCR-amplified. These two procedures have been united into one technique known as RT-PCR (Israeli et al. 1994).

RT-PCR has also been used to determine the distribution patterns of MOR splice variants in rat brain (Zimprich et al. 1995) and lymphoid tissue (Pampusch et al. 1998). Primers are designed to unique sequences within each splice variant and RTPCR is performed on isolated sections of total RNA (e.g. RNA isolated and extracted from the frontal cortex or cerebellum etc). This technique is unable to provide morphological localisation data and does not entirely represent the amount of mRNA to be transcribed to the active protein.

RT-PCR has been used to identify variant mRNA isoforms of human KOR (hKOR) and murine DOR (mDOR). The RT-PCR products generated from the KOR and DOR mRNAs were of a larger size than was expected, thus prompting DNA sequencing of the RT-PCR products, revealing new putative splice variants of hKOR and mDOR (Gaveriaux-Ruff et al. 1997).

The main advantages of RT-PCR are the efficiency and speed with which one can achieve results. Primers are designed specifically to the sequence of interest and are thus very specific. RT-PCR is a sensitive technique that is capable of amplifying rare and low frequency mRNA transcripts.

The major disadvantage of RT-PCR is non-specific amplification, which occurs as a result of either mispriming or amplification from partially digested or undigested genomic DNA (Komminoth et al. 1994). Modifications have been made to try and 
overcome these problems, such as hot-start PCR and DNase pre-treatment of the sample (Nuovo 1994) but these new applications have failed to completely overcome the non-specific amplification produced by RT-PCR.

RT-PCR has been used to identify mRNA distribution patterns in situ, where RTPCR is performed followed by a separate ISH step (Ertsey and Scavo 1998), which has the disadvantage of being extremely time consuming.

More recently a direct in situ RT-PCR (IS-RT-PCR) method has been described with an enhanced sensitivity, which boasts all the benefits of RT-PCR with new methods to overcome the disadvantages (Kher and Bacallao 2001). By using fluorescently labelled primers in the in situ PCR mix one is able to directly detect the cellular localisation of the mRNA of interest, thus eliminating the lengthy ISH step. A specific restriction enzyme (designed to cut genomic DNA and not primer sequence) is used in conjunction with the DNase digestion step before RT-PCR to reduce the amount of non-specific amplification. One disadvantage of this technique has been the inefficiency of signal amplification and therefore low level mRNA detection (Mee et al. 1997).

\subsubsection{Fluorescent in situ Hybridisation (FISH)}

In situ hybridisations were initially performed using radioactive labelled probes and therefore autoradiography to detect the localisation of the hybrids. The transition to using fluorescently labelled probes has vastly increased the variety of applications of ISH technology. The use of these fluorescently labelled probes for in situ hybridisation is termed FISH. The use of FISH is growing rapidly in genomics, 
cytogenetics, gene mapping and biomedical research. This technique has major advantages over the aforementioned methods, including increased spatial resolution, reduced turn around time for results, and the capability of simultaneous detection of multiple transcripts using differentially labelled probes. To date there are no publications reporting the use of FISH to determine the distribution patterns of the MOR splice variants. FISH was the main technique used in the present study and is therefore covered in more detail in Chapter 3.

\subsection{Aims of Thesis}

The initial aim of the present study was to exploit the robustness and specificity of circularisable ligation probes (CLiPs) in a unique approach of in situ genotyping the MOR splice variants (refer Chapter 2). CLiPs were designed to target the exon boundaries of MOR splice variants. They were labelled with the fluorophore Fluorescein-dUTP (F-dUTP) in order to enable direct visualisation of the morphological localisation of MOR splice variants in rat brain, using ISH. Ultimately it was hoped that CLiPs would be able to be utilised for genotyping MOR splice variant mRNA in situ.

However, CLiPs were moved away from in this project due to time restraints and inherent limitations (discussed in Chapter 2). A more conventional technique fluorescent in situ hybridisation (FISH) was used to analyse MOR splice variant distribution, as FISH was an established technique in the laboratory. The use of FISH to determine MOR splice variant distribution, not only set up an alternative experimental approach, but also enabled one to test whether the levels of MOR mRNA were sufficient for visualisation. If FISH was successful and the 
hybridisation conditions for short labelled-riboprobes could be elucidated, then it was considered feasible for CLiPs to be able to hybridise and ligate to MOR splice variants in situ.

Therefore the aim became to produce RNA probes (riboprobes) approximately the same size as the target specific region of CLiPs $(\sim 60-70 \mathrm{nt})$ to analyse the distribution patterns of MOR splice variants in rat brain. Four short $(\sim 70-95 \mathrm{nt})$ riboprobes were generated to exons 1,3,4 and 9, and the 5' UTR + exon 1 of the Rattus norvegicus Oprm gene. The short length of each riboprobe represented a unique parameter for this project and is discussed in detail in Chapter 3. The riboprobes were directly labelled with fluorescent-NTPs to allow for the direct visualisation of MOR splice variants in rat brain tissue sections using FISH. 


\section{Chapter 2}

\section{Analysis of MOR Splice Variants using Circularisable Ligation Probes (CLiPs)}

\section{$\underline{2.0 \text { Introduction }}$}

Circularisable Ligation Probes (CLiPs), also known as Padlock Probes (Wu and Wallace 1989, Nilsson et al. 1994, Nilsson et al. 1997, Baner et al. 1998) or C-probes (Zhang et al. 1998a) are single-stranded oligodeoxyribonucleotides (oligos) that contain target specific regions of 15-30 nucleotides (nt) at both ends, separated by a non-homologous linker region of $>50$ nts. Once a CLiP is bound to its target, the 5', and 3' ends are juxtaposed forming a nick that may be ligated by the action of DNA ligase, causing the CLiP to become topologically locked (Figure 2.0).

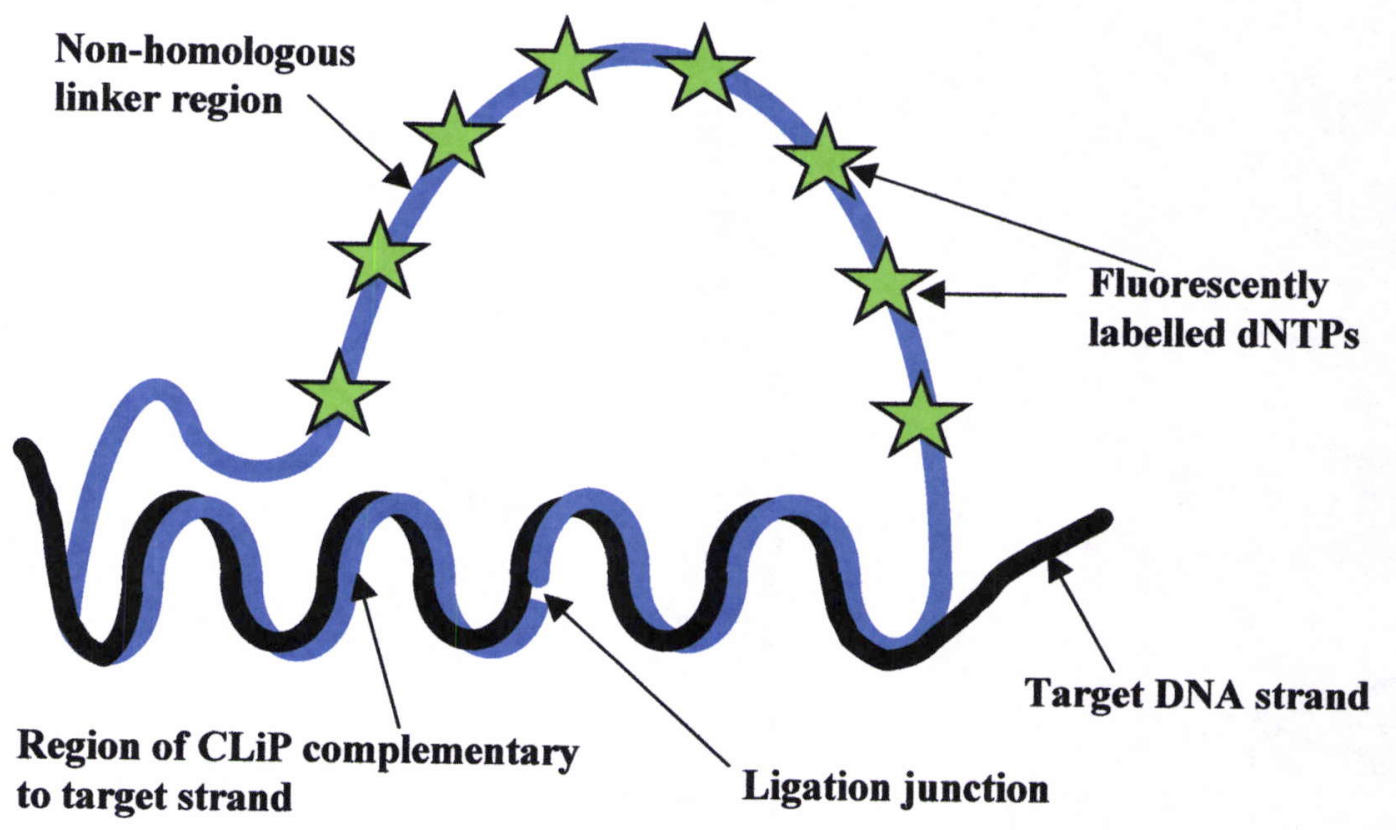

Figure 2.0 Schematic representation of CLiP structure hybridised and ready for ligation (adapted from Landegren et al. 1996). Due to the helical nature of DNA CLiPs are wound around the target strand causing them to be topologically locked to their target via catenation. The incorporation of fluorescently labelled dNTPs allows for direct visualisation. 
Traditionally CLiP production has been achieved using chemical synthesis techniques. One of the many limitations of chemical synthesis is the difficulty faced in generating CLiPs of $>100$ nts. This is due to the proportion of incorrect nucleotides incorporated with increasing length. Recently a PCR-based approach has been utilised for enzymatic CLiP synthesis (Myer and Day 2001).

In 1994 Newton et al. showed that any short sequence of DNA could be incorporated into a PCR product by the addition of this sequence as a 'tail' to the 5 ' end of a primer. As the additional sequence has little effect on specificity or efficiency of hybridisation, it is merely incorporated into the product and amplified by PCR (Newton and Graham 1994). This technique has been exploited in cloning technologies, whereby a primer is tailed with restriction endonuclease sites to allow for digestion, producing 'sticky ends' which increase the efficiency of cloning (Scharf et al. 1986).

CLiPs were produced in this project using enzymatic CLiP synthesis, whereby the primers used contained a $5^{\prime}$ tail complementary to the target gene (Figure 2.1).

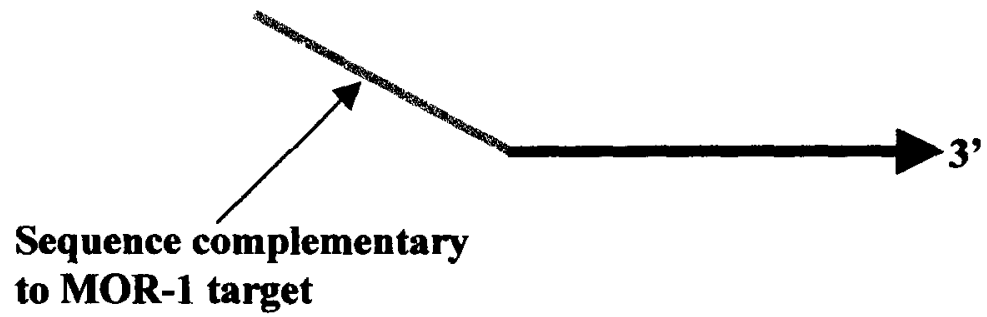

Figure 2.1 Schematic representation of the primer structure designed for CLiP synthesis. The $5^{\prime}$ end of the primer (grey) is complementary to the MOR-1 target sequence while the $3^{\prime}$ end (black) is complementary to the non-homologous pBSSK DNA used to form the linker region. During PCR the $5^{\prime}$ target sequence is incorporated into the PCR products. 
The primer itself (i.e. minus the tail) amplifies off a non-homologous template to form the linker region of the CLiP. The first round PCR results in a product that contains both the primer sequence and the sequence of the 5' 'tail' (Figure 2.2).

A.

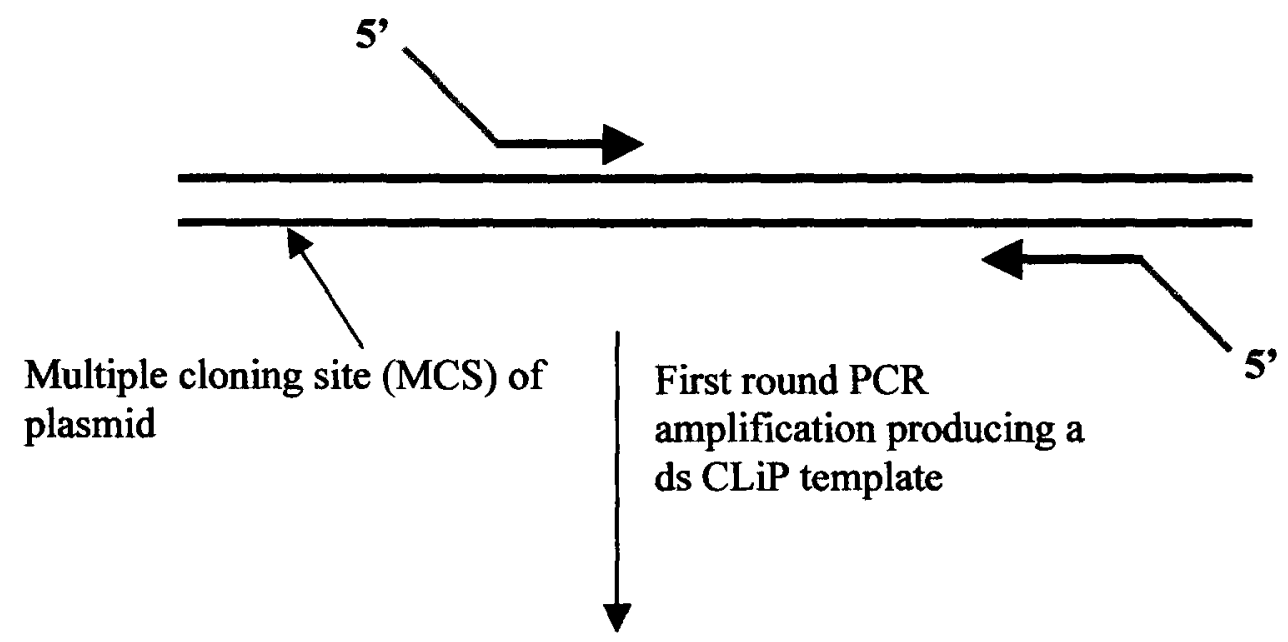

B.

Figure 2.2 Schematic representation of ds CLiP template synthesis. A. Primers containing the 5' MOR-1 target sequence tail are used to amplify off a linearised plasmid generating the non-homologous region of the CLiP. B. The resulting product incorporates both primers and their complementary sequences (represented by dashed orange line).

This product (hereafter referred to as ds CLiP template) is purified and used in the second round CLiP-forming PCR. CLiPs require a 5' phosphorylated end at the nick junction in order for ligation to occur, this is achieved by phosphorylation of the forward primer that is used in second round PCR.

In this project ss CLiPs were captured from the second round PCR product using streptavidin-coated paramagnetic beads (SA-beads). Affinity capture using SAbeads requires the addition of a biotin molecule to one strand of the PCR product. This was achieved by 5 ' biotinylation of the reverse primer, which was used in 
second round PCR. After PCR amplification the resultant products contained both a 'top' 5'phosphorylated strand and a 'bottom' 5' biotinylated strand, hereafter this product is referred to as a ds CLiP (Figure 2.3).

A.

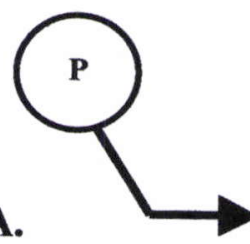

B.

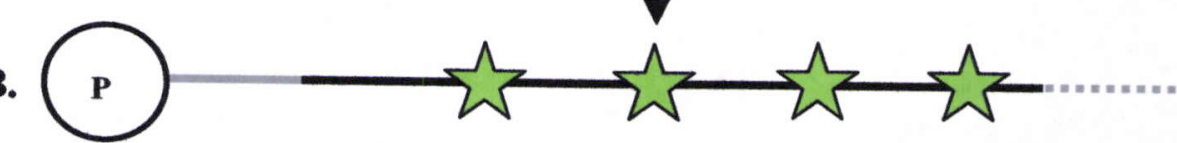

Exponential amplification of $\mathrm{ds}$ CLiP by PCR with F-dUTPs

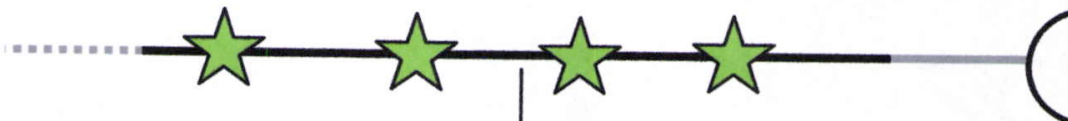

B

The ds CLiP is immobilised on SA-beads via the 5'-biotin and the complementary strand is released by alkali elution.

C.

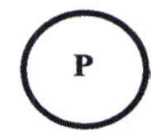

Figure 2.3 Schematic representations of enzymatic CLiP synthesis and ss CLiP purification using affinity capture. A. Two primers are used to amplify a ds CLiP template by PCR. The 5' phosphorylated (P) forward primer defines the 5' end of the ss CLiP. The reverse primer is $5^{\prime}$ biotinylated $(B)$ for affinity capture. $B$. The ds PCR product is labelled with F-dUTPs $(\vec{\xi})$, this product is immobilised on SAbeads via the 5 '-biotin. C. The complementary strand forming the ss CLiP is released by denaturation with $\mathrm{NaOH}$. 
The second round PCR is performed in the presence of fluorophore-labelled dNTPs. Fluorescein-modified dUTP (F-dUTP) was used in this experiment, as this fluorophore is capable of being directly detected by an automated DNA sequencer and a fluorescent microscope using the correct filter set.

CLiPs are typically 70-100 nt in length and are therefore expensive to produce using chemical synthesis. Producing larger (>100 nt) CLiPs using chemical synthesis results in the production of many truncated sequences, which are difficult to purify from the full-length oligonucleotides (Antson et al. 2000).

The method of enzymatic CLiP synthesis used in this project has several advantages over chemical synthesis. CLiPs larger than 100 nt can be enzymatically synthesised with the well-defined 5' and 3' ends needed for ligation (Myer and Day 2001). Enzymatic CLiP synthesis also allows for the construction of more densely labelled CLiPs at a greatly reduced cost to its chemical counterpart.

Biologically, DNA ligases play an important role in DNA replication, repair and recombination in both prokaryotes and eukaryotes. There are two classes of DNA ligases: $\mathrm{NAD}^{+}$-dependent DNA ligases (found in bacteria), and ATP-dependent ligases (found in eukaryotes, viruses and bacteriophages). Early studies showed that two adjacent synthetic oligos could be joined by the action of a DNA ligase, if guided by a complementary sequence (Wu and Wallace 1989). 
DNA ligation is strongly inhibited by single bp mismatches at either side of the ligation junction (Wu and Wallace 1989) and due to DNA ligases' requirement for perfect hybridisation, CLiPs are able to distinguish target sequence variants, such as single nucleotide polymorphisms (SNPs). SNPs are the most widely distributed genetic markers in the human genome; a polymorphism is defined as a variation of the minor allele at greater than $2 \%$ in any given population (http://www.stats.ox.ac.uk).

In this project ligation was performed using the $\mathrm{NAD}^{+}$-dependent thermostable Thermus thermophilus (Tth) DNA ligase. The mechanism of Tth DNA ligation occurs in three steps: adenylation of the DNA ligase in the presence of $\mathrm{NAD}^{+}$, transfer of the adenylate moiety to the 5'-phosphate of the nicked DNA strand, and attack on the adenylate-DNA bond by the 3'OH of the nicked DNA, sealing the phosphate backbone by the formation of a phosphodiester bond (Luo and Barany 1996). Interestingly, experiments have shown that the error of mismatch ligation increases when the mismatch occurs at the $3^{\prime}$ side of the ligation junction (Wu and Wallace 1989, Luo et al. 1996), this factor was taken into account when designing the CLiPs used in this project.

CLiPs become circularised once they hybridise to their target and if there are no mismatches at the nick junction, CLiPs become topologically "locked" to their target by catenation after ligation. 
The in situ application of CLiPs provides many unique advantages over other localisation techniques currently used (e.g. IHC and FISH). Common problems with these techniques are probe specificity and high signal to noise ratios.

CLiPs have the unique advantage of being topologically locked to their targets, enabling high stringency denaturing washes and exonuclease digestions to be applied, lowering the signal to noise ratio. Exonuclease digestion does not remove ligated CLiPs as they are circularised leaving no free 5' and $3^{\prime}$ ' ends for the exonuclease to digest.

Several other methods are currently used for SNP detection, for example Allele Specific PCR (ASPCR) (Wu et al. 1989) and Ligation Detection Reactions (LDRs) (Barany 1991). CLiPs can be used to genotype SNPs and the requirement for both hybridisation and ligation gives better discrimination of alleles over such techniques as ASPCR. CLiPs can also be utilised for highly multiplex in situ analyses of total genomic DNA or RNA molecules (Landegren and Nilsson 1997).

\subsection{Experimental Design}

The aim of this experiment was to design CLiPs to target exon 1 and 2 boundaries of MOR splice variants (Appendix C, Figure C5). These CLiPs were labelled with the fluorophore F-dUTP in order to enable direct visualisation of the morphological localisation of the MOR splice variant MOR-1 in rat brain, using ISH.

After CLiP production several intermediate experiments were performed, to aid the success of ISH and ligation using CLiPs. Firstly, the CLiPs were ligated in solution 
with a PCR produced MOR-1 template. CLiPs are ss DNA sequences that ligate more efficiently to DNA than mRNA. Therefore the MOR-1 mRNA was to be reverse transcribed to cDNA, to determine whether short (CLiP-length) DNA probes designed to MOR-1 could hybridise in situ. Yet reverse transcription of the MOR-1 mRNA required increased tissue permeability by proteinase $\mathrm{K}$ digestion, resulting in degradation of tissue morphology. Consequently, short DNA probes were to be designed directly to MOR-1 mRNA.

However, because RNA/RNA hybrids are more stable than DNA/RNA hybrids and FISH using riboprobes was an established technique in the laboratory, short fluorescently labelled riboprobes were designed to MOR splice variant sequences to substitute CLiPs.

The use of FISH to determine MOR splice variant distribution, not only set up an alternative experimental approach, but also enabled one to test whether the levels of MOR mRNA were sufficient for visualisation. If FISH was successful and the hybridisation conditions for short labelled-riboprobes could be elucidated, then it was considered feasible for CLiPs to be able to hybridise and ligate to MOR splice variants in situ. Figure 2.4 represents an overview of the experimental design.

Ultimately it was hoped that CLiPs would be able to be utilised for genotyping MOR splice variant mRNA in situ. However, due to time restraints and the inherent limitations of using CLiPs, the more conventional method of FISH was chosen to analyse MOR splice variant distribution. 


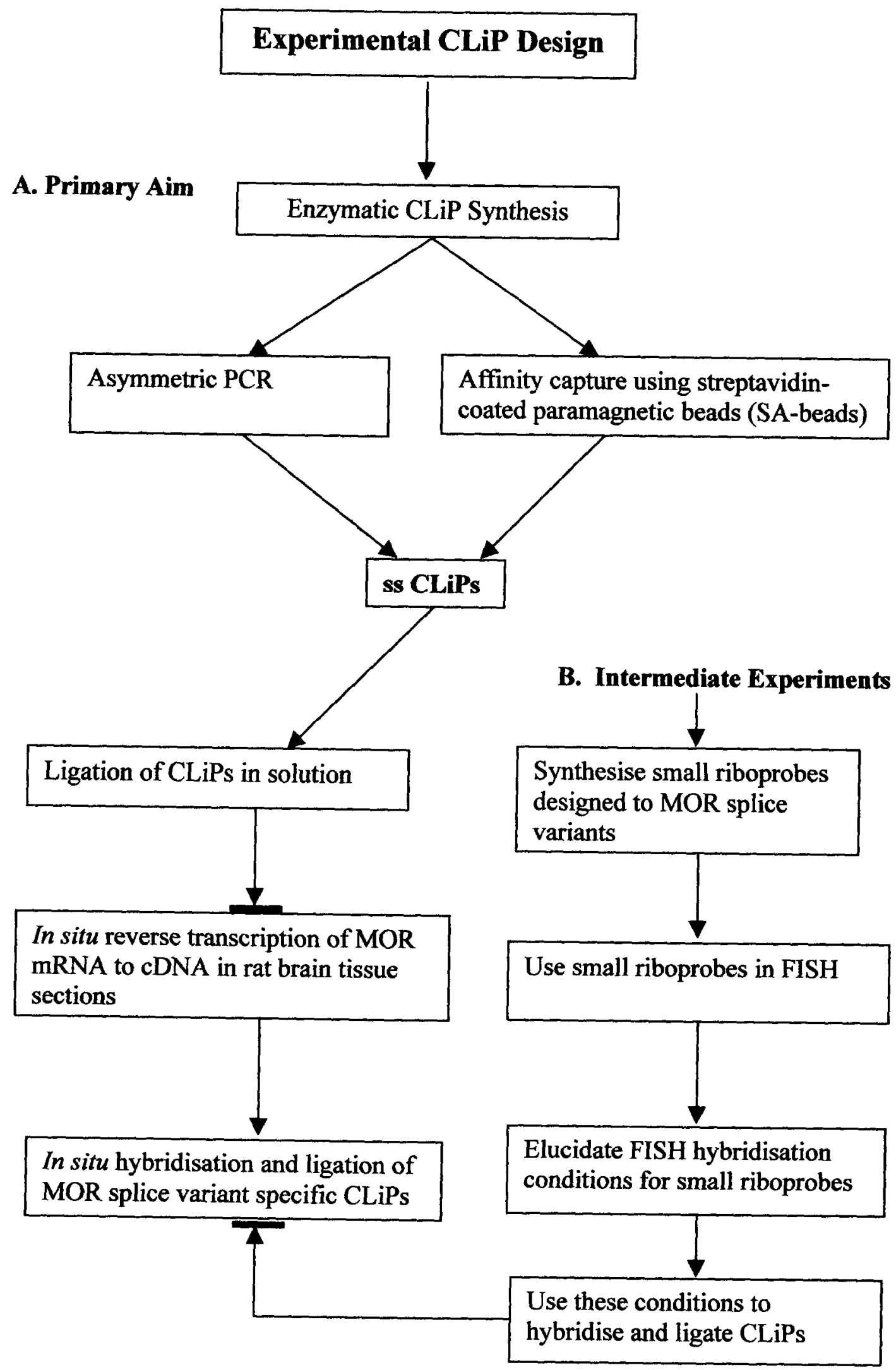

Figure 2.4 Flowchart depicting experimental design of labelled ss CLiPs and the eventual progression to FISH using small riboprobes. Red bars illustrate where experiment was halted due to time constraint. 


\subsection{CLiP Design}

This section presents the design of a CLiP complementary to the Rattus norvegicus MOR-1 splice variant cDNA using two PCR-based methods of ss CLiP synthesis. In designing these CLiPs many factors had to be taken into account. The most critical parameter for successful CLiP synthesis was the design of the primers used to generate CLiPs by the PCR-based method. Several variables were taken into account for primer design, these were: primer length, melting temperature $\left(T_{m}\right), 3$ '-end sequence, GC content and secondary structure formation.

In general oligonucleotides between 18 and 24 bases are extremely sequence specific, provided that the annealing temperature is optimal. The primers used in the CLiP-forming (second round) PCR were both 23 bases in length.

The goal annealing temperature in primer design should be at least $50^{\circ} \mathrm{C}$, as reduced $T_{m}$ generally results in extremely low efficiency, increasing the event of mispriming. In this study the MOR.Fwd primer had a $T_{m}$ of $68.3^{\circ} \mathrm{C}$, and the MOR.Rev primer had a $\mathrm{T}_{\mathrm{m}}$ of $69.1^{\circ} \mathrm{C}$.

It is well established that the 3' terminal position in PCR primers controls the extent of mispriming experienced during PCR (Kwok et al. 1990). Therefore, 3'-end specificity plays an important role in PCR success. In this project it was essential that the 3'-end nucleic acid of the primer was not an adenosine (A), as this would have resulted in the incorporation of a fluorescein-labelled dUTP. F-dUTP is large in size, therefore if it were incorporated on the 3'-end of the CLiP it would have resulted in a large decrease in ligation efficiency due to steric hindrance. 
The recommended base composition of primers is $45 \%-50 \% \mathrm{GC}$, and a $\mathrm{GC}$ content of $>50 \%$ typically results in the formation of stable secondary structures. Additionally a GC content of $\sim 50 \%$ puts the $\mathrm{T}_{\mathrm{m}}$ in an optimal range of around $56^{\circ} \mathrm{C}$ $62^{\circ} \mathrm{C}$ (Dieffenbach et al. 1995). In this study the GC content was $50 \%$ allowing for $50 \%$ AT content, which is sufficient for a significant proportion of the final PCR product (CLiP) to be fluorescently labelled for visualisation. For ease of analysis the forward and reverse primer sequences were combined to form a 46-mer and analysed for $\mathrm{T}_{\mathrm{m}}$ and GC composition. Figure 2.5 summarises the parameters used in CLiP design. The target region of MOR-1 was chosen for two main reasons, firstly the GC composition was $50 \%$ and secondly the sequence lies across the boundary of exons 1 and 2 (see Appendix C for mRNA sequence of MOR-1).

A.

MOR-1 target sequence:

5' AAATCGTCAACGTCTGCAACTGG ATCCTCTCTTCTGCCATCGGTCT 3'

Complementary CLiP sequence:

LR-TTTAGCAGTTGCAGACGTTGACC 5’ 3'TAGGAGAGAAGACGGTAGCCAGA-LR

B.

\begin{tabular}{|c|c|}
\hline Base & Number and Percentage (\%) \\
\hline $\mathrm{A}$ & $14(30.4)$ \\
\hline $\mathrm{C}$ & $8(17.4)$ \\
\hline $\mathrm{G}$ & $15(32.6)$ \\
\hline $\mathrm{T}$ & $9(19.6)$ \\
\hline $\mathrm{A}+\mathrm{T}$ & $23(50.0)$ \\
\hline $\mathrm{G}+\mathrm{C}$ & $23(50.0)$ \\
\hline
\end{tabular}

c.

$\mathrm{T}_{\mathrm{d}}=93.3^{\circ} \mathrm{C}$ (nearest neighbour method)

$\mathrm{T}_{\mathrm{m}}=87.3^{\circ} \mathrm{C}(\% \mathrm{GC}$ method $)$

Figure 2.5 Analysis of CLiP target sequence to region of MOR-1 gene. A. Depicts the MOR-1 sequence and the complementary CLiP sequence. The vertical bar shows where the two ends of the CLiP meet to form the nick junction. LR = linker region. B. This table shows the base composition data of the MOR-1 CLiP, including the \%GC. C. Represents the two optimal temperatures produced by two varying methods. The computer software programme OLIGO6 (Molecular Biology Insights, Inc.) generated data from B and C. 
As the CLiP target gene was mammalian (eukaryotic) in origin (rat Oprm) the first round PCR primers (containing the 5' MOR-1 target sequence tail) amplified off a linearised plasmid (prokaryotic), to form the non-homologous linker region of the CLiP (Figure 2.2). The length of the linker region was varied generating two different sized CLiPs (139nt and $352 \mathrm{nt}$ ); this was achieved by the use of different forward primers. The linker region sequence of each different sized CLiP was analysed for GC content and stable secondary structures (e.g. hairpin formation), to increase the specificity of the CLiPs for the MOR-1 target template.

\section{$\underline{2.3 \text { Single-stranded CLiP Synthesis }}$}

For experiments requiring DNA:DNA hybridisation ss probes are more efficient than ds probes as in solution, probes with complementary sequences (ds probes) are capable of reannealing to themselves. This phenomenon is thermodynamically more favourable than the hybridisation of a CLiP to its target, as the majority of a CLiP (the non-homologous linker region) shares little sequence homology with the target. Therefore attempts to synthesise ss CLiPs were made using primer extension and affinity capture techniques.

\subsubsection{Primer Extension}

Primer extension reactions are the basis of numerous DNA sequencing protocols (Gyllensten and Erlich 1988) and ss probe synthesis techniques (Liu et al. 1986, Konat 1996, Millican and Bird 1997). Primer extension is almost identical to a standard PCR, except the primers are not present in equimolar amounts, the presence of essentially one primer generates the PCR product in a linear rather than exponential fashion. 
The template used for the second round primer extension PCR is depicted in Figure 2.2, B. In the primer extension PCR a 100:1 molar ratio of the forward: reverse primer was used, which resulted predominantly in the amplification of the CLiP forming top strand (Figure 2.6). The small molar amount of the reverse primer was achieved by dilution of the template and therefore carryover into the primer extension reaction. The phosphorylated forward primer (MOR.B1.Fwd, refer Table 2.0) hybridised to the template and extension was achieved using a DNA polymerase and thermal cycling (Figure 2.6).

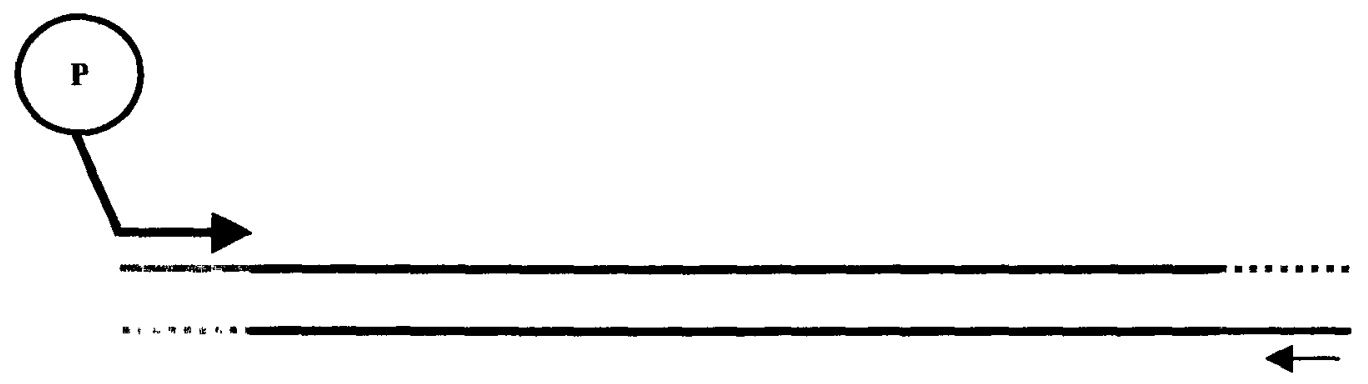

Figure 2.6 Schematic representation of primer extension. Large arrow depicts the molar excess of the phosphorylated (P) forward primer and the small arrow depicts the carry-over of reverse primer with the template from first round PCR. The solid and dashed orange lines depict incorporation of the MOR-1 target sequence and its complementary sequence respectively.

The amount of PCR product produced by primer extension is reliant mainly on the number of cycles and the amount of template used. Ss CLiPs were generated using 35 thermal cycles and approximately $4 \mathrm{ng}$ of the CLiP template (refer Experimental Procedures). The approximate final yield per reaction was $50 \mathrm{ng}(1 \mathrm{ng} / \mu \mathrm{L})$. While ss CLiPs were successfully produced by this method, ligation attempts in solution were unsuccessful. This experiment was run briefly and in conjunction with the more successful affinity capture technique. 


\subsubsection{Affinity Capture}

Affinity capture is a technique frequently used for the production of ss DNA, which can be used in DNA sequencing (Hultman et al. 1989), This method typically exploits the strong interactions between streptavidin and biotin (Chaiet and Wolf 1964). Streptavidin is a biotin-binding protein found in the culture broth of Streptomyces avidinii. Streptavidin binds 4 molecules of biotin per tetrameric protein molecule with extremely high affinity $\left(\mathrm{K}_{\mathrm{d}} \sim 10^{-15}\right)$.

In this project, the reverse primer used in second round PCR was biotinylated. PCR amplification using the biotinylated reverse primer resulted in a ds PCR product (ds CLiP) with one strand end-labelled with biotin (Figure 2.3). Purification of this product was necessary prior to affinity capture with the streptavidin-coated paramagnetic beads (SA-beads), as an excess of unincorporated biotin-labelled primers increases SA-bead saturation.

The ds CLiPs were purified by gel excision after agarose gel electrophoresis, to remove the unincorporated primers as well as other spurious bands. The ds CLiP product of correct size was gel excised and solubilised with the oxidising agent sodium perchlorate and added directly into washed SA-beads (refer Experimental Procedures, Section 4.4.1). The purified ds CLiPs were immobilised using SA-beads via the 5'-biotin and the complementary strand (ss CLiP) was released by alkali elution (Figure 2.3). 


\subsection{Ligation of CLiP}

Ligation of MOR-1 specific CLiPs was achieved by hybridisation and ligation to a MOR-1 target template in solution. This target template was a $794 \mathrm{bp}$ fragment of the MOR-1 gene generated by PCR (refer Experimental Procedures).

CLiP ligation was achieved by thermal cycling with 40 Units of Tth DNA ligase, where each ligation reaction contained approximately $8 \mathrm{ng}$ of the labelled CLiP and 0.5 pmol of the MOR-1 target.

The ligation reactions were analysed using $4 \%$ polyacrylamide gel electrophoresis (PAGE) on an ABI PRISM ${ }^{\circledR} 377$ DNA Sequencer using GeneScan ${ }^{\circledR}$ Analysis Software. Ligated products were differentiated from linear unligated probes due to the altered mobility of circularised CLiPs.

\subsection{Results and Discussion}

\section{Generation and ligation of ss CLiPs using primer extension}

Primer extension reactions were used to produce ss CLiPs. Figure 2.7 shows 2 bands representing the ds CLiP templates used in the primer extension reactions. The two ds CLiP templates are shown in Figure 2.7, lane 1 (139 bp) and lane 2 (352 bp).

An approximate final yield per reaction tube was 1-2 $\mu \mathrm{g}(20-40 \mathrm{ng} / \mu \mathrm{L})$; the amount of PCR product was estimated from a known standard using agarose gel electrophoresis (Figure 2.7). 


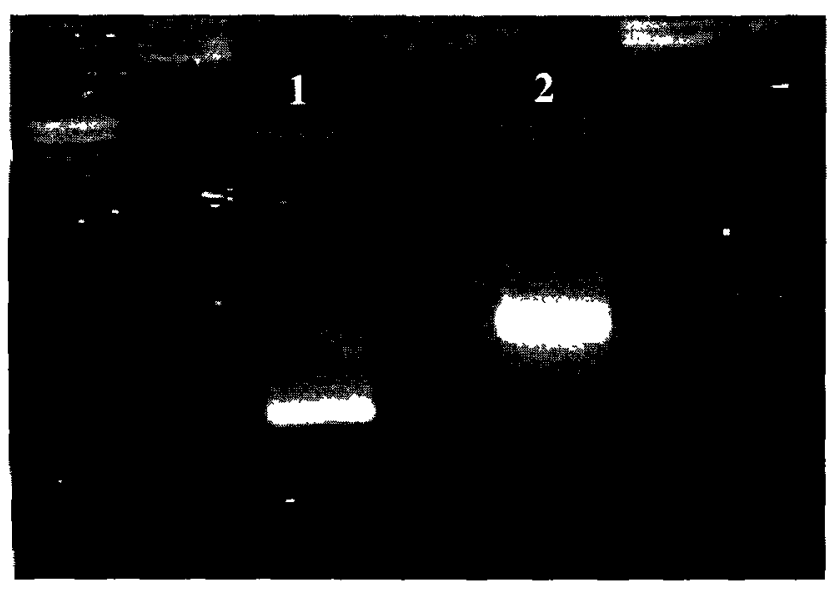

Figure 2.7 Synthesis of ds CLiP template. Varying forward primers were used to generate different size linker regions with the same MOR-1 target sequence on both products. 1. Shows the ds CLiP product that is $139 \mathrm{bp}$ in size. 2. Shows the ds CLiP product that is $352 \mathrm{bp}$ in size. Faint lower band is unincorporated primers. $1 \mathrm{~kb}+$ ladder (Invitrogen ${ }^{\mathrm{TM}}$ Iffe technologies) was included alongside samples to allow size determination and product yield estimation. Amplifications were performed as detailed in Chapter 4, Experimental Procedures. Analysis of products was performed by agarose gel electrophoresis using a $2.5 \%$ agarose gel. Visualisation was achieved using a UV transilluminator after staining the product with $320 \mathrm{ng} / \mathrm{mL}$ ethidium bromide (Chapter 4, Experimental Procedures).

PCR products generated from first round PCR (Figure 2.7) were purified by gel extraction (refer Experimental Procedures). The approximate yield of each purified PCR product was $500 \mathrm{ng}(10 \mathrm{ng} / \mu \mathrm{L})$; these products were used as template for ss CLiP synthesis by primer extension.

Primer extension PCRs were set up using two different concentrations of the phosphorylated forward primer $(200 \mathrm{nM}$ and $400 \mathrm{nM})$ to determine if various concentrations could produce more ss CLiPs (Figure 2.8). 


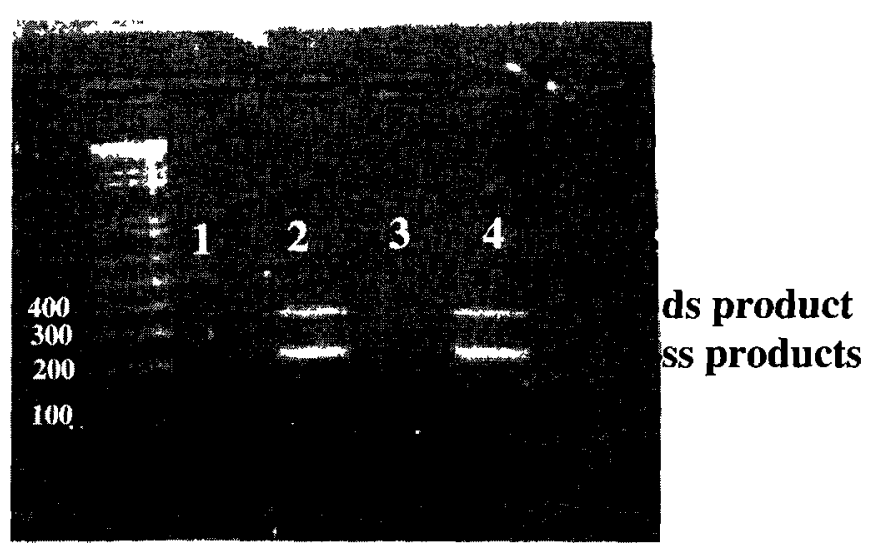

Figure 2.8 Synthesis of ss CLiPs using primer extension PCR. Primer extension was carried out using $\sim 10 \mathrm{ng}$ of the correct purified ds CLiP template (i.e. ds CLiP $139 \mathrm{bp}$ or ds CLiP $352 \mathrm{bp}$ ) and varying concentrations of the forward primer. 1. $200 \mathrm{nM}$ MOR.B1.Fwd + ds CLiP template 139. 2. $200 \mathrm{nM}$ MOR.B1. Fwd + ds CLiP template 352. 3. $400 \mathrm{nM}$ MOR.B1.Fwd + ds CLiP template 139. 4. 400 $\mathrm{nM}$ MOR.B1.Fwd + ds CLiP template 352. $1 \mathrm{~kb}+$ ladder (Invitrogen ${ }^{T M}{ }_{\text {life technologies }}$ ) was included in the far left lane to allow size determination in bp. Amplifications were performed as detailed in Chapter 4. Analysis of PCR products was performed by agarose gel electrophoresis using a $2.5 \%$ agarose gel. Visualisation was achieved using a UV transilluminator after staining the product with $320 \mathrm{ng} / \mathrm{mL}$ ethidium bromide (Chapter 4, Experimental Procedures).

Figure 2.8 shows the production of two bands following primer extension and that varying the forward primer concentrations had little effect on the amount of product produced. It was determined that the smallest bands were the ss CLiP products of interest, as ss DNA is smaller (e.g. 139 nts instead of 139 bp). The largest bands however could have been a number of different products. It is most likely that these bands were the template used in the primer extension reactions, however the largest bands could have been new ds CLiP products caused by a large carryover of the reverse primer with the template. This is unlikely as the template (shown in Figure 2.7) was gel excised, in order to rid the template of unincorporated primers and dNTPs (seen as faint bands at the bottom of the gel, Figure 2.7).

An experiment was set up to determine the origin of the top band. Assuming it was carryover of the template, the PCR was set up with and without the DNA polymerase 
Platinum $^{\circledR}$ Taq. This PCR experiment also investigated varying the amounts of dNTPs (using either $50 \mu \mathrm{M}$ or $200 \mu \mathrm{M}$ dNTPs in each PCR) to investigate whether altering the amount of nucleotides would increase the amount of ss CLiP produced (Figure 2.9).

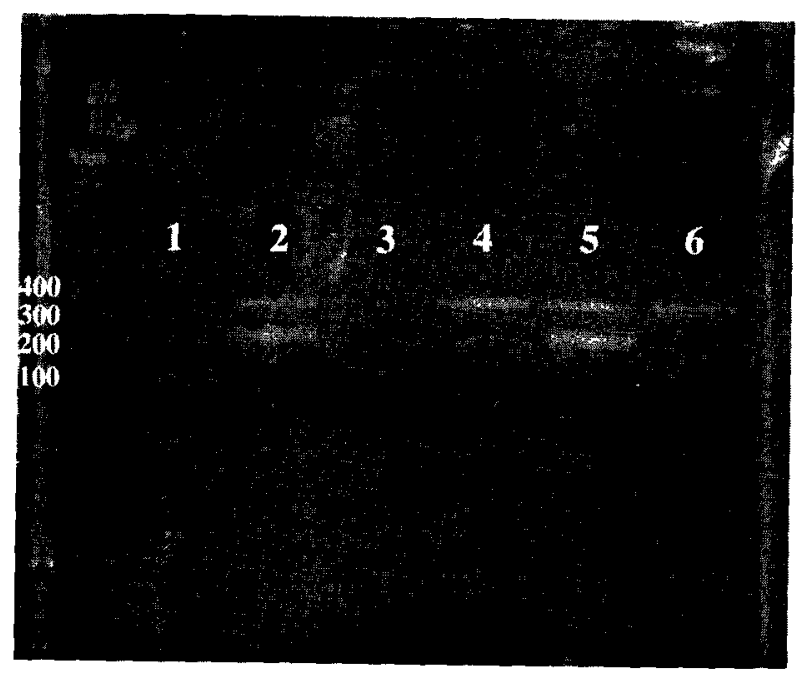
Lane
1. + P.Taq, $50 \mu \mathrm{M}$ dNTPs
2. +P.Taq, $50 \mu \mathrm{M}$ dNTPs
3. - P.Taq, $50 \mu \mathrm{M}$ dNTPs
4. - P.Taq, $50 \mu \mathrm{M}$ dNTPs
5. + P.Taq, $200 \mu \mathrm{M}$ dNTPs
6. - P.Taq, $200 \mu \mathrm{M}$ dNTPs

Figure 2.9 Optimising primer extension PCR. This involved the presence $(+)$ or absence (-) of Platinum ${ }^{\circledR}$ Taq (P.Taq) DNA polymerase and varying amounts of dNTPs (see text box). Lanes 1 and 3 represent the smaller $139 \mathrm{nt}$ CLiP and lanes 2,4,5 and 6 represent the larger $352 \mathrm{nt}$ CLiP. $1 \mathrm{~kb}+$ ladder (Invitrogen ${ }^{T M}{ }_{\text {life technologies }}$ ) was included in the far left lane to allow size determination in bp. Analysis of PCR products was performed by agarose gel electrophoresis using a $2.5 \%$ agarose gel. Visualisation was achieved using a UV transilluminator after staining the product with $320 \mathrm{ng} / \mathrm{mL}$ ethidium bromide (refer Experimental Procedures).

The primer extension PCRs set up without Platinum ${ }^{\circledR}$ Taq (Figure 2.9, lanes 3, 4 and 6) reveal the presence of a single band, that corresponds in size and amount to that of the original template added (10 ng of purified ds CLiP template). The primer extension PCRs set up with Platinum ${ }^{\circledR}$ Taq (Figure 2.9, lanes 1,2 and 5) reveal the presence of two bands. Comparison of these two experiments led to the conclusion that the bottom most band was the expected ss CLiPs. As there appeared to be no real difference in the amount of ss CLiP produced using either $50 \mu \mathrm{M}$ or $200 \mu \mathrm{M}$ dNTPs, the lesser amount of $50 \mu \mathrm{M}$ was used in all following primer extension PCRs. 
As the primer extension PCR succeeded in producing ss CLiPs, the experiment was repeated with the incorporation of the fluorophore F-dUTP to enable direct visualisation of the ss CLiPs (Figure 2.10). A labelling ratio of 1:10 FdUTPs:dTTPs was used in the primer extension PCR, as this had previously been determined as a labelling ratio that provided sufficient sensitivity and produced a high yield of labelled PCR products (Myer 2001).

The primer extension reaction for the larger $352 \mathrm{nt}$ CLiP produced more product than the $139 \mathrm{nt} \mathrm{CLiP}$, and as the ss $352 \mathrm{nt}$ CLiP was easier to distinguish on a $2.5 \%$ agarose gel (Figure 2.9 Lanes 2 and 5) the labelled primer extension PCR reactions were set up solely for the $352 \mathrm{nt}$ CLiP (Figure 2.10).

Figure 2.10 shows one product at the size expected for the ds CLiP template (352 bps), however upon gel inspection using a UV transilluminator, a faint green band could be seen running at the same size as the band visible in Figure 2.10. This represents the successful production of labelled ss CLiPs. The incorporation of large F-dUTPs results in the ss CLiPs appearing green under UV luminescence and running at a much larger size than would be expected (Figure 2.10). 


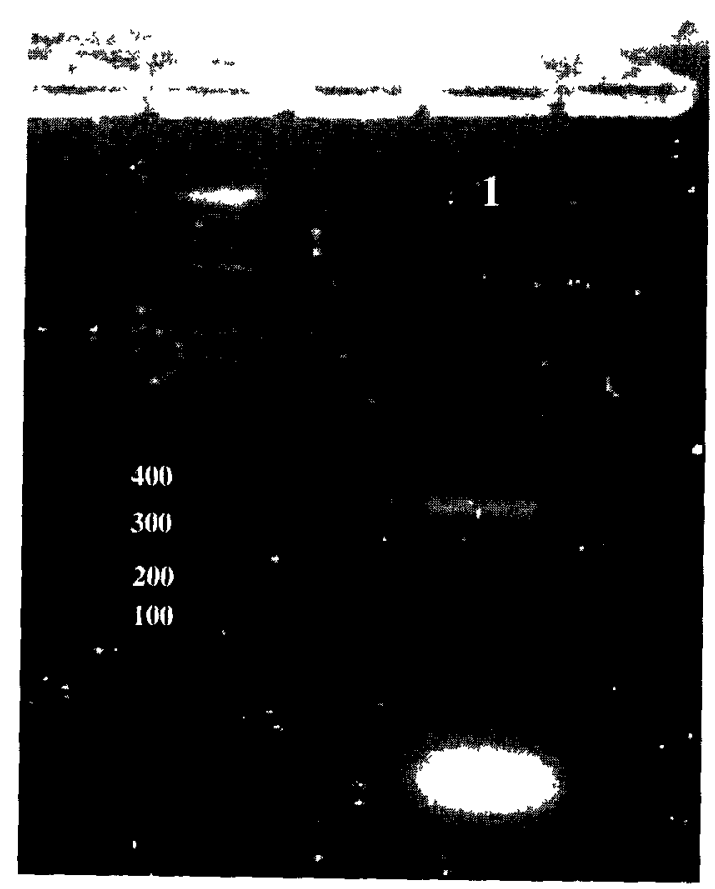

Figure 2.10 Labelled primer extension PCR, producing F-dUTP labelled ss CLiPs. 1. The product generated is estimated to be $352 \mathrm{bp}$ in size [estimation

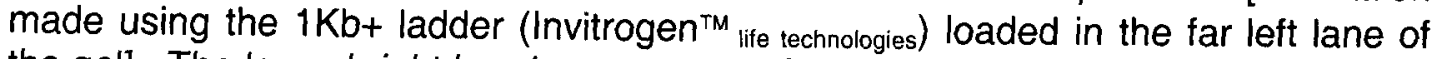
the gel]. The lower bright band represents unincorporated F-dUTPs. Amplifications were performed as detailed in Chapter 4, Experimental Procedures. Analysis of PCR products was performed by agarose gel electrophoresis using a $2.5 \%$ agarose gel. Visualisation was achieved using a UV transilluminator after staining the product with $320 \mathrm{ng} / \mathrm{mL}$ ethidium bromide (refer Experimental Procedures).

The ss CLiPs produced by primer extension (above) were purified by gel excision and used in a ligation reaction in solution (refer Experimental Procedures). The ligation reactions were analysed on an ABI PRISM ${ }^{\circledR} 377$ DNA Sequencer using GeneScan ${ }^{\circledR}$ Analysis Software. Ligated products were differentiated from linear unligated probes due to the altered mobility of circularised CLiPs.

Unfortunately the ss CLiPs produced in this project using primer extension PCR, were never successfully ligated to the MOR-1 target template in solution. By analysing the GeneScan ${ }^{\circledR}$ Chromatograms it was possible to see a peak only at 352 nt, representing the linear unligated CLiP. As ligated CLiPs show retarded mobility when analysed by denaturing PAGE, they are easily differentiated from unligated 
CLiPs (Nilsson et al. 1994, Kwiatkowski et al. 1996). When CLiPs were produced using primer extension PCR, two peaks were not visible on the GeneScan ${ }^{\circledR}$ chromatograms.

This led to the conclusion that the CLiPs produced by primer extension were unable to ligate to their target template. One possible explanation is that the Platinum ${ }^{\circledR}$ Taq DNA polymerase (used during the first round ds CLiP template PCR) caused the addition of non-templated nucleotides and therefore the alteration of the target specific sequence of the CLiPs. It is more likely that the forward primer became dephosphorylated, causing the Tth ligase to be unable to ligate the nick junction, preventing CLiP circularisation and ligation.

\section{Generation and ligation of ss CLiPs using affinity capture}

Affinity capture was used to produce ss CLiPs, as this approach had been successful in our laboratory in the past (Myer and Day 2001). Again 139 bp and 352 bp ds CLiPs were produced, shown in Figure 2.11 in Lanes 1 and 2, respectively (refer Experimental Procedures).

The ds CLiP PCR was set up without labelled F-dUTPs first, to ensure the PCR conditions produced at least $20-50 \mathrm{ng}$ of ds CLiPs. This PCR was repeated including F-dUTPs (with a labelling ratio of 1:10 F-dUTPs:dTTPs) to produce labelled ds CLiPs (Figure 2.11). 


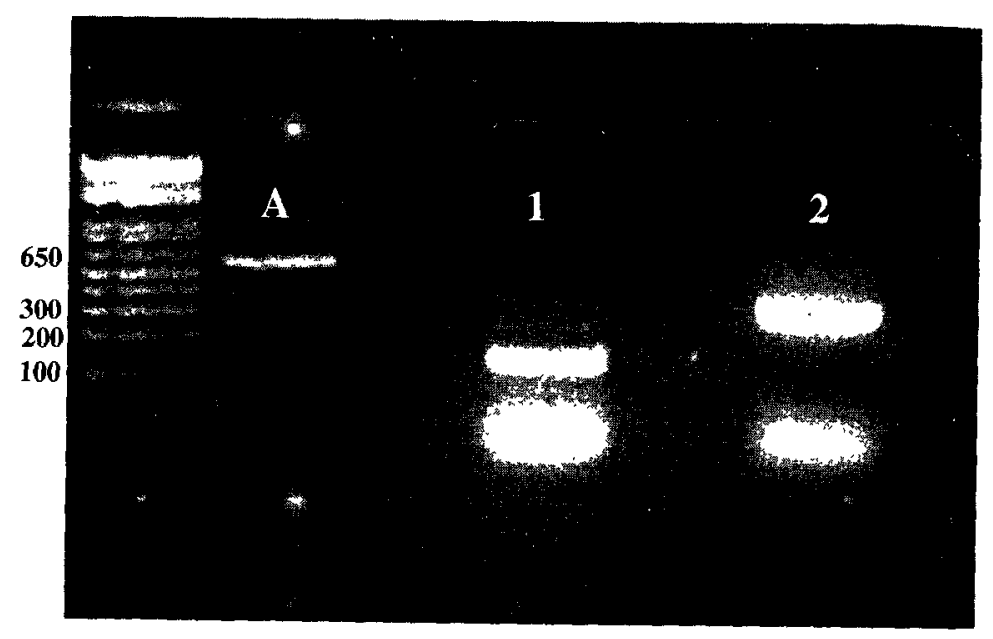

Figure 2.11 Second round PCR producing labelled ds CLiPs.

A. PCR-generated MOR-1 target template. $1 \mathrm{~kb}+$ ladder (Invitrogen $^{\top \mathrm{M} M}$ life technologies) was included in the far left lane to allow size determination in bp. 1. Small $139 \mathrm{bp}$ labelled ds CLiP. 2. Larger 352 bp labelled ds CLiP. Both ds CLiP products 'glow' brightly due to F-dUTP incorporation. The lower bright band represents unincorporated F-dUTPs. Amplifications were performed as detailed in Chapter 4, Experimental Procedures. Analysis of PCR products was performed by agarose gel electrophoresis using a $2.5 \%$ agarose gel. Visualisation was achieved using a UV transilluminator after staining the product with $320 \mathrm{ng} / \mathrm{mL}$ ethidium bromide (refer Experimental Procedures).

The ds CLiP products shown in Figure 2.11 were purified to remove excess unincorporated primers and dNTPs. PCR product purification was achieved by either gel excision or PCR purification, depending on what the purified products were to be used for (refer Experimental Procedures). Gel excision was the method commonly used. The band of the correct size was excised from the agarose gel, and solubilised using sodium perchlorate, then the ds CLiP was bound to SA-beads to trap the product. The SA-beads initially failed to capture the ds CLiP product, as no ss CLiP could be recovered by $\mathrm{NaOH}$ elution. One possible reason the SA-beads failed to capture the biotinylated strand could have been potential SA-bead contamination with a biotin-containing substance, blocking the available binding sites. 
New SA beads were bought and used in parallel experiments with the old SA beads, to deduce if the old SA beads being used were no longer suitable (i.e. contaminated). Previous experiments showed that agarose gel solubilisation with the oxidising agent sodium perchlorate was preferential over other purification techniques such as phenol/chloroform extractions, as the ss CLiPs could be captured directly from the solubilised mixture, saving time (Myer 2001).

As no ss CLiPs were being eluted, supernatants from the affinity capture were run on an agarose gel to determine where the ds CLiPs were being lost (refer Experimental Procedures). This required a different purification technique as gel excision and solubilisation results in high levels of sodium perchlorate being present in the supernatants, making the sample unable to be examined by agarose gel electrophoresis, because the presence of sodium perchlorate in the supernatant solubilises the gel. Therefore the ds CLiPs were purified using a PCR purification kit (refer Experimental Procedures). The supernatants and eluate were run on a $2.5 \%$ agarose gel, this revealed that the ds CLiPs were present in the supernatants of both the old and new beads and that no products could be seen in the eluate lanes.

In this case, the most likely reason for ds CLiPs not binding the SA-beads was a poorly synthesised biotin-labelled primer. Primer synthesis could have been checked using MALDI-TOF MS, to determine the percentage of biotin coupling on the primer, however this method is time consuming and expensive, therefore the cheaper option was to order a new biotinylated primer. 
The new biotinylated primer was used to produce F-dUTP-labelled ds CLiPs. These ds CLiPs were purified by gel excision and used in affinity capture. This time the beads captured the biotinylated strand and ss CLiPs were eluted. An aliquot of the eluate was run on a $2.5 \%$ agarose gel shown below. Figure 2.12 shows the two different sized ss CLiP products (139 nt Lane 1 and 352 nt Lane 2), as with Figure 2.10 these bands appear to be running at a much larger size than would be expected for ss CLiP products. During agarose gel inspection using a UV transilluminator both products faintly fluoresced due to the incorporation of large F-dUTPs, causing the ss CLiPs to appear larger than would be expected (Figure 2.12).

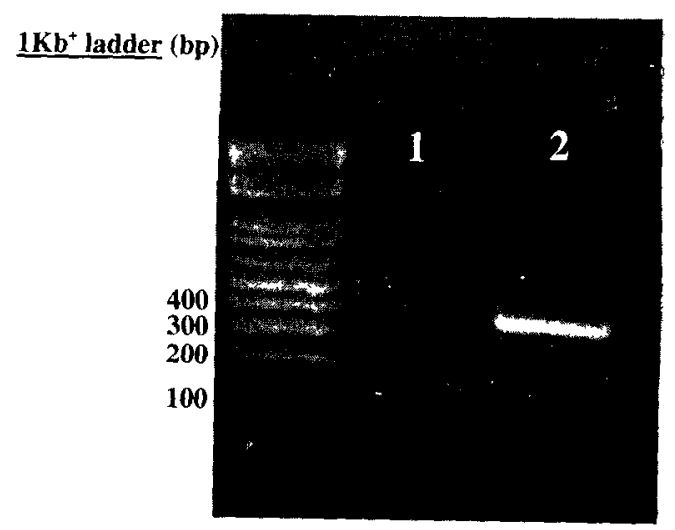

Figure 2.12 Eluate from affinity capture containing ss CLiPs. $5 \mu \mathrm{L}$ out of a total volume of $25 \mu \mathrm{L}$ was loaded per lane on this gel. 1. Small $139 \mathrm{nt}$ SS CLiPs. 2. Large $352 \mathrm{nt}$ ss CLiPs. Analysis of PCR products was performed by agarose gel electrophoresis using a $2.5 \%$ agarose gel. Visualisation was achieved using a UV transilluminator after staining the product with $320-\mathrm{ng} / \mathrm{mL}$ ethidium bromide (refer Experimental Procedures). An approximate final yield of the $139 \mathrm{nt}$ ss CLiPs was 2 $\mathrm{ng}$ and the $352 \mathrm{nt}$ ss CLiPs was $4 \mathrm{ng}$, the amount of ss CLiP product was estimated from a known standard using agarose gel electrophoresis.

Ss CLiPs were quantified with a Molecular Devices SpectraMAX Gemini using the fluorescent dye SYBR Green II (refer Experimental Procedures) for single stranded products. The eluate containing ss CLiPs was used either directly in a ligation reaction or desalted then dried in a vacuum centrifuge. Use of the vacuum dried CLiPs was achieved by resuspension in TE buffer (refer Experimental Procedures). 
The template for ligation was generated by PCR from a linearised plasmid containing a cloned excerpt of the MOR-1 gene (courtesy of Bronwyn Kivell). The ligation reaction was set up using $8 \mathrm{ng}$ of the labelled ss CLiP and $0.5 \mathrm{pmol}$ of the PCR-generated MOR-1 target template (Figure 2.11, A). The ligation reactions were analysed on the ABI PRISM ${ }^{\circledR} 377$ DNA Sequencer using GeneScan ${ }^{\circledR}$ Analysis Software (Figure 2.13).

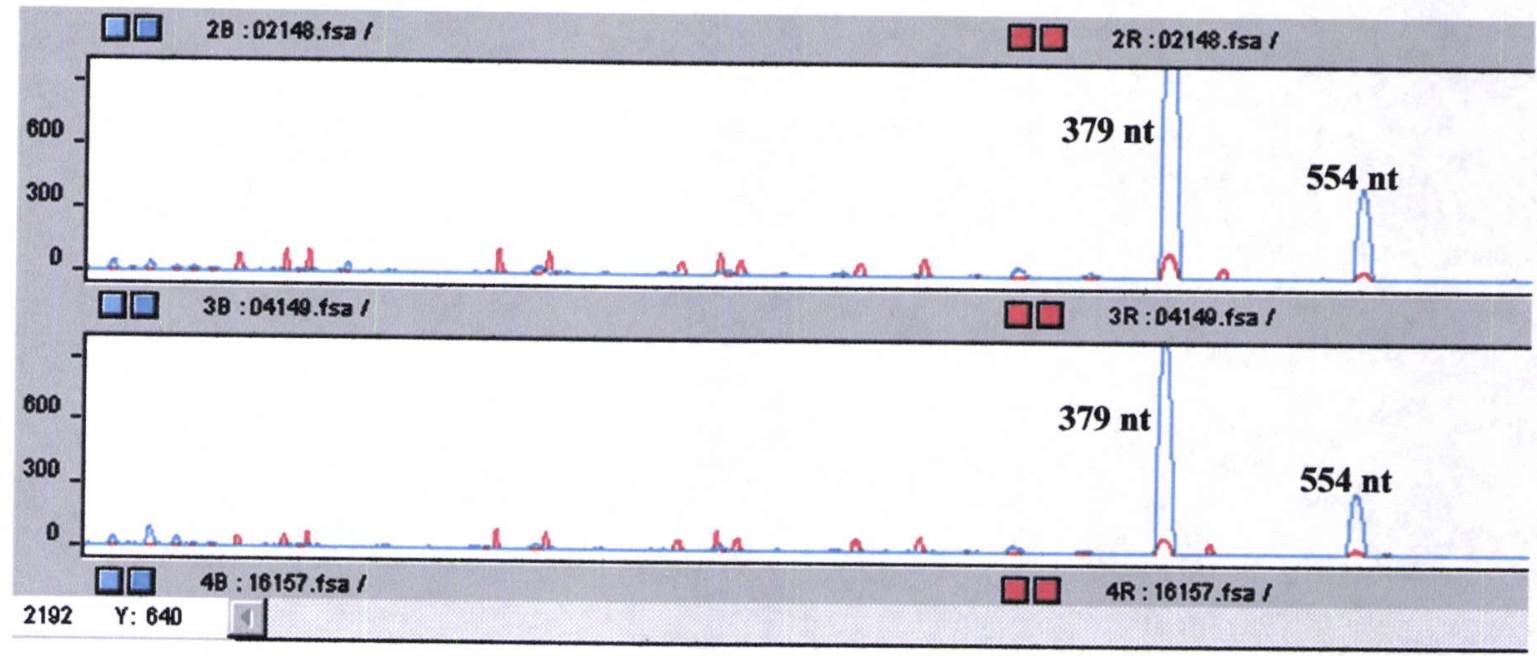

Figure 2.13 Analysis of ligation reactions on the ABI PRISM ${ }^{\circledast} 377$ DNA Sequencer using GeneScan ${ }^{\oplus}$ Analysis Software version 3.7. The size of the CLiPs were determined by comparison to the GeneScan ${ }^{\mathrm{TM}}-2500$ ROX size standard represented by small red peaks. The fluorescence signal intensity is represented on the $y$-axis and the mobility is represented of the $x$-axis. The blue peaks at $379 \mathrm{nt}$ represent the linear $352 \mathrm{nt}$ CLiPs (running at a slightly larger size due to the incorporation of large F-dUTPs and it is assumed that the peak shown at $554 \mathrm{nt}$ is the circularised CLiPs.

Figure 2.13 represents two ligation reactions analysed using the GeneScan software. Circularised CLiPs exhibit a higher mobility when analysed on a denaturing PAGE gel. An uncircularised CLiP is expected to produce one peak at the corresponding size (e.g. $352 \mathrm{nt}$ ), whereas circularised CLiPs are expected to produce two peaks, one at the actual size of the CLiP ( $352 \mathrm{nt})$ and another between $500-600 \mathrm{nt}$ (circularised). The results shown in Figure 2.13 are consistent with a circularised CLiP. 
Therefore it is likely that CLiPs designed in this project were specific to the MOR-1 template. As much difficulty was experienced trying to synthesise and ligate CLiPs to the MOR-1 splice variant target template, the more conventional method of FISH was used to analyse MOR splice variant distribution in rat brain.

\subsection{Conclusions}

To determine if CLiPs generated in this project ligated to their target, more experimentation would be required. Control experiments should be set up simultaneously with each ligation reaction, such as performing the ligation reactions with no target DNA or an incorrect but similar target (e.g. DOR cDNA).

To confirm that the CLiPs utilised in the project did circularise, an exonuclease digestion of the ligation reaction should be performed. Circular DNA molecules are resistant to exonuclease digestion, due to the absence of free 5' and 3' ends. Therefore if the secondary peak shown if Figure 2.13 were to disappear after an exonuclease digestion, this would suggest that these CLiPs had not circularised. Future possibilities for this technique would be to ligate CLiPs in situ to reverse transcribed MOR-1 cDNA in rat brain. One method could be to hybridise CLiPs to their reverse transcribed cDNA target at the correct stringency, using a hybridisation buffer containing deionised formamide. This buffer could be washed off and CLiPs ligated to their target in a ligation buffer. Alternatively CLiPs could be hybridised in ligation buffer so both reactions could take place at the same time. Ultimately this technique is feasible yet technically difficult. 
Chapter 3

\section{Analysis of MOR Splice Variant Distribution Using Fluorescent in situ Hybridisation (FISH)}

\section{$\underline{\text { 3.0 Introduction }}$}

\section{In situ hybridisation (ISH)}

ISH was introduced in 1969 (Gall and Pardue 1969a, John et al. 1969), it is a technique used for the morphological localisation of genetic sequences. ISH exploits the fundamental properties of nucleic acids to anneal to one another a phenomenon called hybridisation. Hybridisation is the reaction whereby two ss nucleic acid molecules can recognise one another by the formation of a hydrogen bond between two complementary base pairs (Henderson 1996). This is not only true for DNADNA duplexes, but also for DNA-RNA and RNA-RNA combinations. Labelled RNA probes can be designed to hybridise to a complementary sequence of mRNA within a tissue section (e.g. brain). Probes designed to mRNA in particular can be used to determine information about gene expression, for example the gene expression patterns (distribution) as ISH can provide semi-quantitative data about gene activation or suppression, for example, by following the changes in mRNA expression levels during development (Buono and Lang 1999).

Single-stranded RNA probes used in ISH experiments must be labelled to allow for detection of the nucleic acid sequence of interest. The most recent advances in this field have been due to the development of new probe labelling molecules [e.g. fluorescein isothiocyanate-UTP (FITC-UTP) or digoxygenin-UTP (DIG-UTP)] that have replaced radioactively labelled ribonucleotides. 


\section{$\underline{3.1 \text { Fluorescent in situ hybridisation (FISH) }}$}

FISH entails the labelling of the DNA or RNA probes with reporter molecules. FISH was initially introduced as a method of gene mapping (Cheung et al. 1977) and has had profound effects on efforts to detect, map and characterise human disease genes such as acute myeloid leukaemia (AML) (Gozzetti and Le Beau 2000). FISH has also had a major impact in the research fields of early human development (Delhanty et al. 1997), animal cytogenetics (Sebastian et al. 1997) and comparative genomic mapping between species (Frengen et al. 1997).

The advantages of using FISH to map the MOR splice variants over the other techniques described in Chapter 1 , Section 4 are listed below. FISH is a highly specific technique that can be performed very rapidly, allowing larger numbers of cells to be scored simultaneously. The efficiency of hybridisation and signal detection for a carefully designed probe using FISH is very high, enabling the direct correlation of morphological features within a tissue section to expression patterns of the DNA, mRNA or protein of interest. Furthermore, by combining differentially labelled probes designed to discriminate between various regions of mRNA (e.g. different exons of a gene) one is able to obtain a multitude of data from one experiment. For example experiments could be performed to determine whether MOR splice variants are co-localised within the same cell.

\section{Probe Types}

The disadvantages of FISH vary depending mainly on probe selection. Several different types of probes can be used, each with their own advantages and disadvantages (summarised in Table 3.0). The probe types are ds DNA, ss DNA, ss 
RNA and oligonucleotides. Single-stranded RNA probes are also called complementary RNA (cRNA) probes or in this project riboprobes. It should be noted that riboprobe ${ }^{\circledR}$ is a registered trademark of the Promega Corporation.

\begin{tabular}{|c|c|c|}
\hline Probe Type & Advantages & Disadvantages \\
\hline Double-stranded DNA & $\begin{array}{l}\text { High specific activity } \\
\text { Subcloning unnecessary } \\
\text { Possibility of signal amplification } \\
\text { Easy to use }\end{array}$ & $\begin{array}{l}\text { Being ds DNA probes can reanneal } \\
\text { to one another (decreasing probe } \\
\text { availability) } \\
\text { Probe denaturation is required } \\
\text { DNA-DNA hybrids are less stable } \\
\text { than RNA-RNA hybrids }\end{array}$ \\
\hline Single-stranded DNA & $\begin{array}{l}\text { No probe denaturation needed } \\
\text { No reannealing during } \\
\text { hybridisation }\end{array}$ & $\begin{array}{l}\text { Technically complex } \\
\text { Subcloning generally required } \\
\text { Hybrids less stable than riboprobes }\end{array}$ \\
\hline Riboprobe & $\begin{array}{l}\text { Stable RNA-RNA hybrids } \\
\text { High specific activity } \\
\text { No probe denaturation needed } \\
\text { No reannealing during } \\
\text { hybridisation } \\
\text { Unhybridised riboprobes can be } \\
\text { enzymatically destroyed } \\
\text { (RNases), sparing hybrids } \\
\text { Good tissue penetration if made a } \\
\text { small size }\end{array}$ & $\begin{array}{l}\text { Subcloning generally required (not } \\
\text { necessary) } \\
\text { Difficult to work with as } \\
\text { riboprobes are very sensitive to } \\
\text { RNases (ubiquitous RNA } \\
\text { degrading enzymes) }\end{array}$ \\
\hline Oligonucleotide & $\begin{array}{l}\text { No cloning required } \\
\text { Stable } \\
\text { Good tissue penetration if made a } \\
\text { small size } \\
\text { No self-hybridisation }\end{array}$ & $\begin{array}{l}\text { Limited labelling methods } \\
\text { Lower specific activity, therefore } \\
\text { less sensitive } \\
\text { Less stable hybrids than riboprobes }\end{array}$ \\
\hline
\end{tabular}




\section{Probe Labels and Labelling Methods}

Radiolabelled probes are generated by the incorporation of radioactive isotopes (e.g. ${ }^{32} \mathrm{P},{ }^{35} \mathrm{~S}$ or ${ }^{3} \mathrm{H}$ ) into the nucleic acid sequence of the probe. Radiolabelled probes are sensitive and can detect low-level transcripts present in small amounts. Radiolabelled probes used in ISH reactions can be detected using autoradiography, this method of detection allows for signal quantification using silver grain counting techniques. However, radiolabelled probes are less popular today than they have been in the past due to the issue of safety when using radioactive isotopes, and due to recent advances in non-radiolabelled reporter molecules.

The use of non-radiolabelled probes allowed for the development of FISH. Signal detection methods vary depending on which reporter molecule a riboprobe is labelled with. Direct detection is possible when a flurophore such as Cyanine3-UTP (CY3UTP) is incorporated during riboprobe synthesis, such that after probe hybridisation and post-hybridisation washes have been applied, the probe can be immediately visualised under the fluorescence microscope.

Indirect procedures require the labelled riboprobe to be detected using immunocytochemical techniques. A riboprobe can be labelled with molecules such as biotinylated-UTPs and detected indirectly using the fluorescein-avidin - antiavidin system. Indirect labelling systems have the disadvantage of requiring signal amplification cascades and therefore are more time consuming. Also amplification steps result in an increase in signal intensity that is not directly proportional to the relative abundance of the target mRNA. 
There are five main methods of labelling nucleic acid probes, namely: Nick translation, primer extension, end läbelling (e.g. terminal deoxynucleotidyl transferase), direct labelling or the use of RNA polymerases.

T7 RNA polymerase is a highly specific DNA-dependent RNA polymerase from the E. coli bacteriophage T7. This polymerase has an extremely high specificity for T7 promoter sequences. T7 RNA polymerase was used to generate all the riboprobes used in this project. The DNA templates used to make each riboprobe were generated using different methods discussed in more detail in Section 3.2.

In this project riboprobes were designed to the exons $1,3,4,9$ and the 5' UTR + exon 1 of the MOR gene (Oprm), and were utilised to determine MOR splice variant distribution in rat brain using FISH. All riboprobes were directly labelled with the fluorescent-NTPs (FITC-UTP or CY3-UTP) and synthesised using T7 RNA polymerase. As FISH was currently being used in our laboratory all the equipment necessary for this technique was present and the laboratory was RNase-free.

\section{RNase-free Environment}

RNase is a ubiquitous RNA degrading enzyme produced by micro-organisms in the environment and are found on the surface of skin. This enzyme rapidly breaks down mRNAs and riboprobes. An RNase-free environment is very important for the success of FISH experiments. Gloves must be worn at all times to prevent contamination of materials by RNases found on the skin. Solutions must be treated with an RNase inhibitor such as diethylpyrocarbonate (DEPC) and then sterilised by autoclaving. Chemicals that cannot be treated with DEPC (e.g. Tris-HCl) have to be 
handled with baked spatulas and made up in DEPC-treated water. Glassware is rendered RNase free by baking at $200^{\circ} \mathrm{C}$ for 8 hours or more and plasticware (e.g. eppendorf tubes, barrier tips) are bought RNase free.

\section{Tissue Preparation and Treatment}

To preserve tissue morphology in this project the rat was perfused with $4 \%$ paraformaldehyde. The brain was removed, post-fixed in paraformaldehyde then cryo-protected (refer Experimental Procedures) and sectioned on a cryostat ${ }^{1}$. These sections were floated onto glass microscope slides and used in FISH experiments.

Tissue fixation results in the cross-linking of proteins, as the target mRNA is surrounded by proteins, tissue pre-treatments such as permeabilisation are necessary before riboprobe hybridisation. Two common reagents used in tissue permeabilisation are detergents (e.g. Triton $^{\circledR} \mathrm{X}-100$ or SDS) and the enzyme Proteinase K. Lipids were extracted from the tissue section membrane by the addition of Triton ${ }^{\circledR} \mathrm{X}-100$. Proteinase $\mathrm{K}$ is an endopeptidase that non-specifically hydrolyses peptide bonds. This enzyme is used to remove proteins that surround the target mRNA. Paraformaldehyde fixed samples typically require longer digestion periods with Proteinase $\mathrm{K}$.

Another important tissue pre-treatment when using riboprobes is acetylation with acetic anhydride in triethanolamine (TEA). This pre-treatment creates a uniform

\footnotetext{
${ }^{1}$ Whole brain perfusion has recently been replaced by a "fresh-frozen-fix" method in this laboratory, whereby whole brain samples are frozen immediately at $-80^{\circ} \mathrm{C}$, sectioned using a cryostat and fixed for 5 minutes in paraformaldehyde. This new technique has the advantages of: saving time, increasing sensitivity, maintaining tissue morphology (due to no proteinase $\mathrm{K}$ digestion) and producing greater signal.
} 
negative charge across the tissue, which decreases background signals by reducing the amount of non-specific binding. Tissue acetylation also appears to aid RNase inactivation, which may help to produce stronger signals.

\section{Stringency}

Stringency can be defined as: "The conditions of hybridisation that increase the specificity of binding between two single-stranded portions of nucleic acid" (http://users.wmin.ac.uk/ redwayk/lectures/probes.htm). The composition of the hybridisation buffer is critical in controlling the efficiency of the hybridisation reaction. Hybridisation depends on the ability of the ss riboprobe to anneal to the target mRNA just below its melting temperature $\left(T_{m}\right)$. The degree and specificity of binding between two nucleic acid sequences depends on hydrogen bonding between bases on opposite strands and is affected by four parameters:

\footnotetext{
* Temperature

$* \mathrm{pH}$

* Monovalent cation concentration

* Presence of organic solvents (denaturants)
}

The probe length generally determines the temperature used in FISH. In this project temperatures greater than $40^{\circ} \mathrm{C}$ resulted in loss of signal, due to the short length of the riboprobes being used $(\sim 70-90 \mathrm{nt})$. 
Buffers frequently have a $\mathrm{pH}$ of between $6.5-7.5$, but the rate of renaturation is fairly independent of $\mathrm{pH}$. However a higher $\mathrm{pH}$ can be used to increase the stringency of the hybridisation reaction.

Monovalent cations are added typically in the form of $\mathrm{NaCl}+$ sodium citrate (SSC). Free monovalent cations interact electrostatically with the phosphate groups of the nucleic acids decreasing the electrostatic repulsion between the two strands, resulting in an increase in hybrid stability.

The organic solvent used in the hybridisation buffer was formamide, which reduces the thermal stability of duplex sequences allowing hybridisation to be carried out at a lower temperature. In practice the hybridisation is performed at a lower stringency than the posthybridisation washes, this is to allow for hybrid formation during hybridisation. Higher stringency posthybridisation washes are important for removing any non-specifically hybridised riboprobes and leaving only the target specific riboprobes bound.

\section{Controls}

Positive and negative control experiments are extremely important in FISH experiments. Controls create internal guidelines to standardise the evaluation process and empirically establish baselines for data interpretation. Adequate controls must be set up simultaneously with regular FISH experiments, using all the same chemicals and conditions, to ensure that observed labelling is specific to the target sequence (Lewis and Baldino 1990). Controls should include positive and negative samples as well as technical controls to identify false positive and negative results 
(Herrington and McGee 1992). An example of a technical positive control experiment used in this project was the design of a probe to the abundant "housekeeping" gene actin. This experiment tested the quality of the reagents being used and the tissue mRNA.

In this project negative control experiments were set up with each FISH. Examples of the negative control experiments used are listed below:

Hybridisation without any riboprobe and RNase digestion of the mRNA prior to riboprobe hybridisation. RNase digestion of the tissue mRNA is a very common negative control. Once the mRNA is digested and a hybridisation is performed, if the riboprobe does not bind, it can be determined that the probe is specific for mRNA. RNases are notoriously difficult to inactivate and for this reason, it could be argued that the addition of RNase not only digests the mRNA but also the riboprobe, giving a negative result.

In this project, the tissue mRNA was also digested by alkaline hydrolysis using 0.2 $\mathrm{M} \mathrm{NaOH}$ (Figure 3.0). This experiment should give the same results as RNase digestion but was determined to be more credible, as $\mathrm{NaOH}$ is easier to inactivate than RNase. 

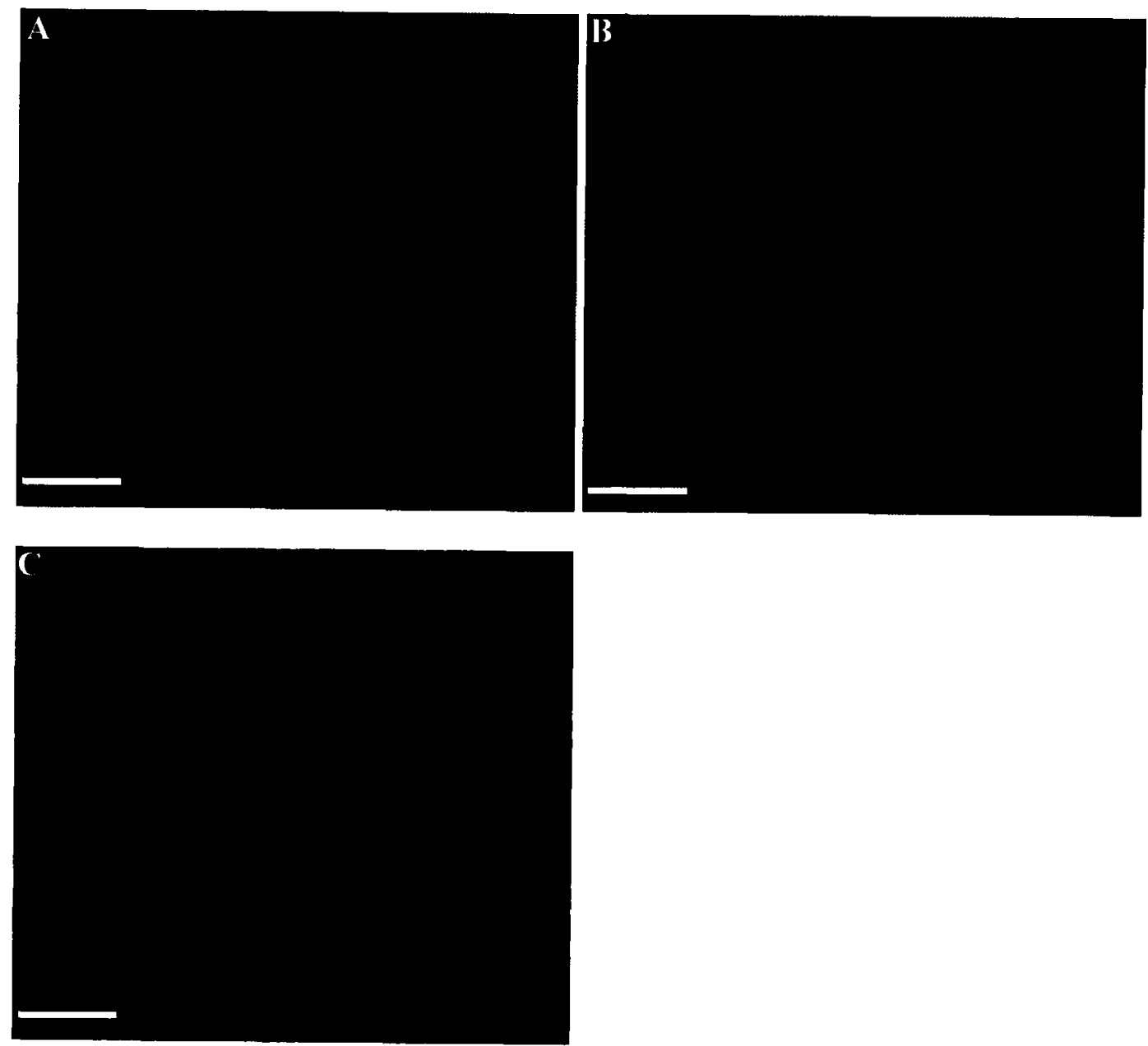

Figure 3.0 Negative control experiment using $\mathrm{NaOH}$-hydrolysed tissue in a FISH. Fluorescent photomicrographs of a sagittal section through the cerebellum. The tissue was $\mathrm{NaOH}$-treated to destroy all RNA before this FISH was performed using a CY3 labelled exon 4 riboprobe confirmed to hybridise specifically (refer section 3.4.10). Images were captured under the CY3 filter set (A), the FITC filter set $(B)$ and the DAPI filter set (C). Panel $\mathbf{C}$ shows the nuclei of the cerebellum. Scale bar $=200 \mu \mathrm{m}$.

Figure 3.0 represents the results of a FISH performed on $\mathrm{NaOH}$-hydrolysed tissue using an exon 4 riboprobe that has been confirmed to be specific for exon 4 of the Oprm. The DNA was not degraded during the $\mathrm{NaOH}$ hydrolysis, as the DAPI nuclear stain is positive for nuclear DNA (Figure 3.0, panel C). Another control experiment is performing the hybridisation with differentially labelled riboprobes to different parts of the same mRNA. This experiment should result in the colocalisation of the riboprobes. 


\section{Guideline for FISH}

An outline of FISH experiments performed in this project is outlined below, for exact guidelines refer to Chapter 4, Experimental Procedures:

The fixed, sectioned rat brain tissue was placed on polylysine-coated microscopic slides, dried and pre-treated to allow for tissue permeabilisation and delipidation etc. The dye SYBR Green II (Molecular Probes) was used in a semiquantitative assay to determine the concentration of each riboprobe (refer Experimental Procedures). Based on this assay $6 \mathrm{ng}$ of riboprobe was added to each hybridisation reaction. The hybridisation buffer and $6 \mathrm{ng}$ of riboprobe was added to a special hybridisation chamber (formed by an adhesive "in situ frame") and left to hybridise to their targets overnight at a set and constant temperature, typically the overnight hybridisation temperature was $40^{\circ} \mathrm{C}$.

The in situ frame was removed and posthybridisation washes were applied with increasing stringency to remove any non-specific hybrids. The sections were washed for $2 \times 15$ minutes at $37^{\circ} \mathrm{C}$ in $2 \times \mathrm{SSC}$ and then for $2 \times 15$ minutes at $37^{\circ} \mathrm{C}$ in 1x SSC. Subsequently slides were washed for 20 minutes at $37^{\circ} \mathrm{C}$ in $2 \mathrm{x} \mathrm{SSC}$ containing $40 \%(\mathrm{v} / \mathrm{v})$ deionised formamide. Nuclei were stained by a 20 minute wash at $40^{\circ} \mathrm{C}$ in $0.5 \mathrm{x}$ SSC containing $5 \mathrm{ng} / \mathrm{mL}$ DAPI. An antifading reagent was added to each slide to reduce the amount of photobleaching experienced during fluorescent microscopy. The slides were viewed using a fluorescent microscope fitted with the appropriate narrow band filters specific for each fluorophore used, allowing for direct visualisation of riboprobes and nuclei (if a fluorescent nuclear stain was used). 


\subsection{Experimental Design}

This section presents the design, synthesis and purification methods used to generate four short ( 70-95 nt) riboprobes to exons 1, 3, 4 and 9, and the 5' UTR + exon 1 of the Rattus norvegicus Oprm gene. The short length of each riboprobe represented a unique parameter for this project and is discussed in detail below. The riboprobes were directly labelled with fluorescent-NTPs to allow for the direct visualisation of MOR splice variants in rat brain tissue sections using FISH. All primer sequences used to generate these riboprobes are given in Table 4.0.

\subsubsection{Riboprobe Design and Synthesis}

Each riboprobe was designed based on a critical set of parameters for oligonucleotide probes (Erdtmann-Vourliotis et al. 1999) (Table 3.1). The computer software programme OLIGO6 (Molecular Biology Insights, Inc.) was used to design the PCR primers used to generate each riboprobe.

\begin{tabular}{|l|l|}
\hline Parameter & Range \\
\hline GC content & $48-62 \%$ \\
\hline Length & $42-54$ nucleotides \\
\hline Hairpin & 0 to $-4.7 \mathrm{kcal}$ \\
\hline Length of runs & Up to 5 \\
\hline
\end{tabular}

Table 3.1 The critical parameters used to design PCR primers for riboprobe synthesis, adapted from Erdtmann-Vourliotis et al. 1999.

Riboprobe length is a very important parameter in probe design. Large riboprobes, greater than 300-500 nt in length, are more tolerant to mismatches than short riboprobes (e.g. a short riboprobe with 2 mismatches is less likely to hybridise to its target than a long riboprobe with 2 mismatches). Riboprobes too large in size tend to have poor tissue penetration. However the amount of label that each large riboprobe molecule can carry is increased, therefore increasing signal. Long riboprobe 
production is more efficient than short riboprobe production, for this reason, long riboprobes are frequently generated and subsequently hydrolysed into shorter fragments.

One aim of this project was to produce riboprobes approximately the same size as the target specific region of CLiPs ( 60-70 nt). Short riboprobe $(70-100 \mathrm{nt})$ have the advantages of being more stable with time (i.e. longer shelf life), easier and cheaper to make, and they hybridise more rapidly than longer riboprobes. The addition of 1 -

3 fluorescently labelled nucleotides is sufficient for probe visualisation under a fluorescent microscope. This is proven by the success of the terminal deoxynucleotidyl transferase end labelling method. A disadvantage of using short riboprobes can be a decrease in sensitivity due to the limited amount of labelled nucleotides incorporated.

\section{Exon 3 riboprobe}

Exon 3 of the Oprm is present in all the MOR splice variants (Figure 1.3). To generate the ds DNA template required for exon 3 riboprobe synthesis, a mammalian expression vector (refer Chapter 4, Experimental Procedures) was obtained containing the MOR-1 gene (courtesy of Bronwyn Kivell). This plasmid was purified and linearised by restriction digestion to be used as template in a PCR. The primers for this PCR were designed to exon 3 using the parameters shown in Table 3.1. The $\mathrm{T} 7$ promoter sequence was added to the reverse primer sequence, allowing for $\mathrm{T} 7$ promoter sequence incorporation into the PCR product (Figure 3.1). This PCR product was then used for the T7 RNA polymerase-catalysed synthesis of exon 3 riboprobes. 
A.

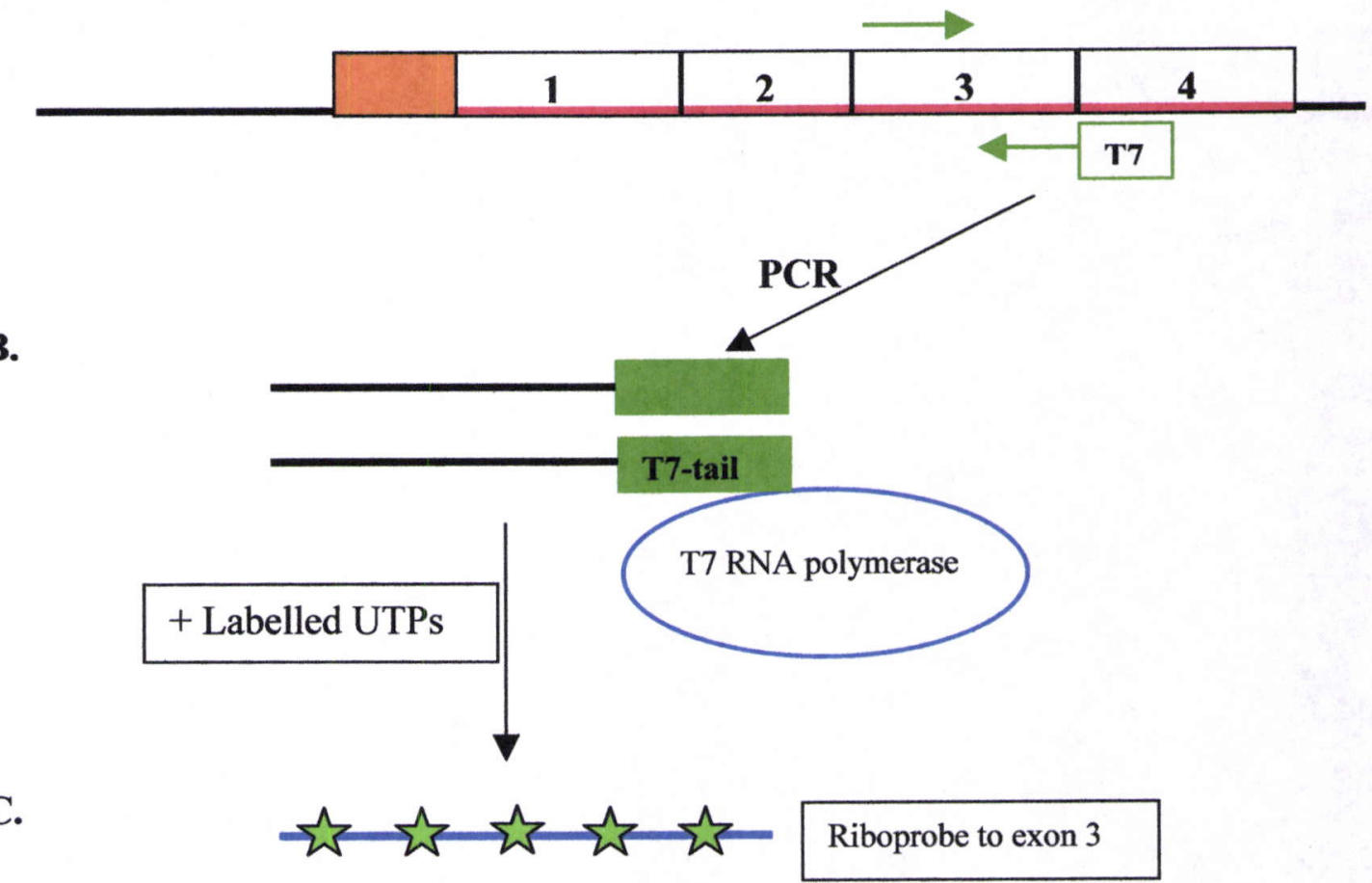

Figure 3.1 Schematic overview of exon 3 riboprobe synthesis (not drawn to scale). A. Depicts a linearised plasmid (black) containing the MOR-1 gene (red). The MOR-1 gene is inserted into the multiple cloning site of the plasmid. Green arrows represent the exon 3 primers, the reverse primer contains the T7 promoter sequence. The orange box at the beginning of exon 1 represents the $5^{\prime}$ untranslated region (UTR). B. Represents the DNA template of exon 3 after PCR the green blocks depict the incorporation of the T7-tail sequence and the complementary sequence on the top strand. Once this product is purified, riboprobe synthesis is initiated using T7 RNA polymerase (plus labelled-UTPs 2 ).

c. Represents labelled exon 3 riboprobe $100 \mathrm{nt}$ in length.

\section{Exon 4 riboprobe}

Exon 4 of the Oprm is present in 7 of the 15 MOR splice variants, namely: MOR-1, MOR-1G, MOR-1H, MOR-1I, MOR-1J, MOR-1K and MOR-1L. However, MOR$1 G$ - MOR-1L are under the control of the exon 11 promoter and form truncated (possible six transmembrane) MOR receptors (Pan et al. 2001).

Overlapping oligonucleotides (oligos) were used to generate the ds DNA template for exon 4 riboprobe synthesis. These oligos were designed to exon 4 using the 
parameters shown in Table 3.1. Based on the $T_{m}$ of each oligo an overlap of $18 \mathrm{nt}$ was determined to be long enough to generate a ds DNA product $73 \mathrm{nt}$ in length. The reverse oligo was $\mathrm{T} 7$-tailed to allow the incorporation of the $\mathrm{T} 7$ promoter sequence into the ds DNA template.

Klenow fill-in and PCR were trialed simultaneously to determine which method produced the largest amount of correctly sized product. The Klenow fragment is an enzyme from $E$.coli that exhibits the $5^{\prime} \Rightarrow 3^{\prime}$ polymerase activity of DNA polymerase I (pol I), but lacks the $5^{\prime}=>3^{\prime}$ exonuclease activity of intact DNA pol I. The Klenow fragment catalyses the $5^{\prime}=>3^{\prime}$ addition of nucleotides and was used to "fill-in" the overlapping oligos designed to exon 4 . Klenow fill-in produced less ds DNA template than PCR; therefore PCR was used in this project to generate the ds DNA template required for exon 4 riboprobe synthesis (Figure 3.2).

Following PCR the ds DNA template was purified by gel excision after agarose gel electrophoresis. A second round PCR was performed with the exon 4 forward and reverse oligos and the purified template. This PCR was carried out to increase the amount of ds DNA template, as T7 RNA polymerase requires at least $100 \mathrm{ng}$ of DNA template to generate riboprobes. Platinum ${ }^{\circledR}$ Taq DNA Polymerase (Invitrogen ${ }^{\mathrm{TM}}{ }_{\text {life technologies) }}$ ) was the enzyme used in PCR. This enzyme adds nontemplated adenines onto the 5 '-ends of PCR products during amplification. Therefore, before riboprobe synthesis, T4 DNA polymerase was used to polish the ends of the ds DNA template to remove any single-stranded overhangs (Figure 3.2). 
A.

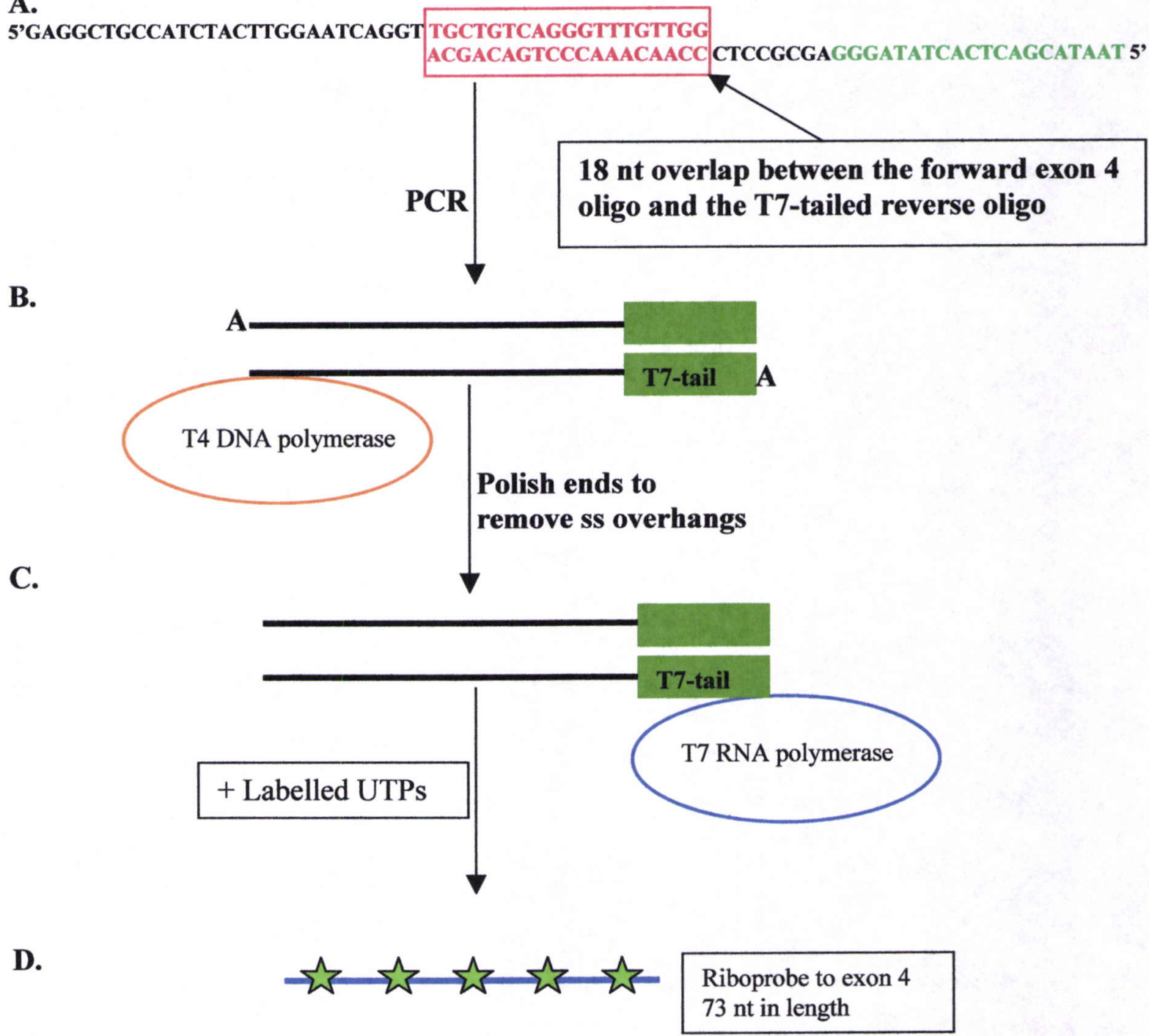

Figure 3.2 Schematic overview of exon 4 riboprobe synthesis (not drawn to scale). A. The 'top' sequence represents the forward oligo and the 'bottom' strand represents the reverse oligo with the T7-tail (Green nt). The red box shows where the two sequences overlap by $18 \mathrm{nt}$. The ds product produced by PCR is purified and used in a second round PCR (not shown in figure). B. Represents the ds DNA template of exon 4 after PCR, the green blocks depict the incorporation of the T7-tail sequence and its complementary sequence on the top strand. This product is polished using T4 DNA polymerase. C. The polished ds DNA template, once this product is purified riboprobe synthesis is initiated using T7 RNA polymerase (plus labelled-UTPs ). D. Labelled riboprobe to exon 4. 


\section{Exon 9 riboprobe}

Exon 9 of the Oprm is present in 6 of the 15 MOR splice variants, namely: MOR1C, MOR-1D, MOR-1E, MOR-1F, MOR-1M and MOR-1N. The ds DNA template for exon 9 riboprobe synthesis was generated by PCR using overlapping oligos. Table 3.1 shows the optimal length of an oligo is $42-54 \mathrm{nt}$, oligos larger than $55 \mathrm{nt}$ result in a higher proportion of incorrect nucleotides being incorporated (Kwiatkowski et al. 1996). To generate the 95 bp ds DNA template for exon 9 riboprobe synthesis, four overlapping oligos (ranging in size from 36-38 nt) were designed (using the parameters shown in Table 3.1) to a region of the Oprm exon 9 gene.

As with the exon 4 riboprobe, PCR was used to fill-in the overlapping oligos (Figure 3.2). PCR was performed and the correctly sized product was purified by gel excision after agarose gel electrophoresis. This product was then used in a second round PCR with two end forming oligos (Figure 3.2A, Oligos A and D) to generate a large quantity of product. The oligo labelled ' $D$ ' in Figure 3.2 was T7-tailed to allow for the promoter sequence to be incorporated into the ds DNA template for riboprobe synthesis.

The ends of the ds DNA product were polished using T4 DNA polymerase to remove any single-stranded overhangs, as T7 RNA polymerase requires ds DNA to initiate transcription. This product was used for the T7 RNA polymerase-catalysed synthesis of exon 9 riboprobes (Figure 3.3). 
A.
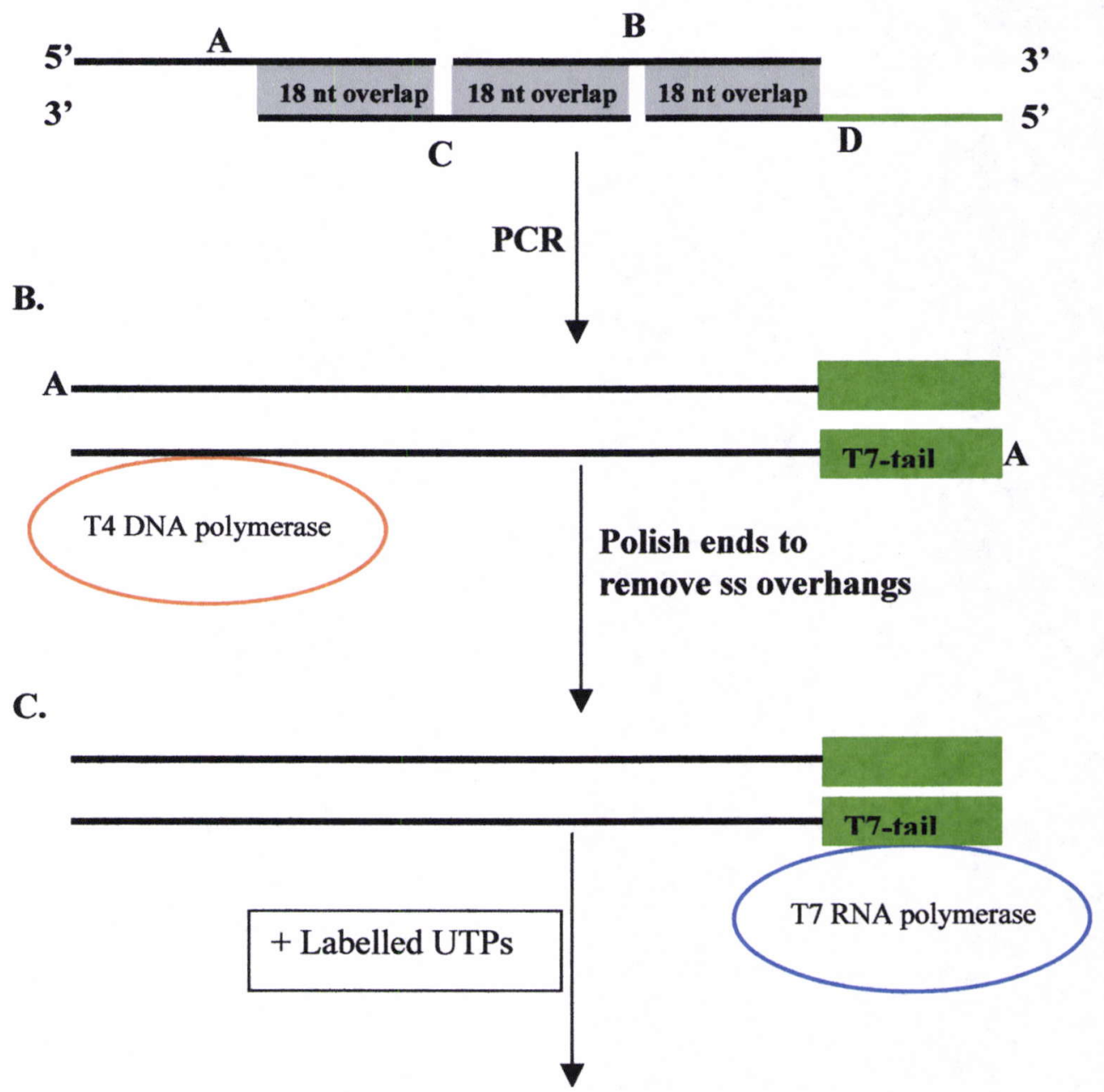

D.

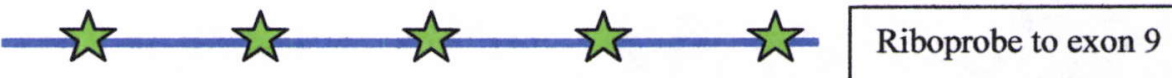

Figure 3.3 Schematic overview of exon 9 riboprobe synthesis (not drawn to scale). A. Shows the four overlapping oligos labelled A - D. The T7-promoter sequence has been attached to the 5'-end of the oligo labelled ' $D$ ' (shown as green line). The grey boxes represent the $18 \mathrm{nt}$ overlap. The ds product produced by PCR is purified and used in a second round PCR (not shown in figure). B. Represents the ds DNA template of exon 9 after PCR, the green blocks depict the incorporation of the T7-tail sequence and its complementary sequence on the top strand. This product is polished using T4 DNA polymerase. C. The polished ds DNA template, once this product is purified riboprobe synthesis is initiated using T7 RNA polymerase (plus labelled-UTPs ${ }^{2}$ ) D. Labelled riboprobe to exon 9.

\section{Exon 1 riboprobe and the 5'UTR + Exon 1 riboprobe}

Exon 1 is present in 10 of the 15 MOR splice variants (namely: MOR-1 - MOR-1F and MOR-1H - MOR1- MOR-1J) of the Oprm. As there are two putative promoters 
of the Oprm, there are two 5' untranslated regions (UTRs). The 5' UTR targeted in this experiment was that of exon 1 not exon 11 . The exon 1 ds DNA template was generated by PCR using a forward primer designed to the 5' UTR (Yoshikawa et al. 1999) and a T7-tailed reverse primer complementary to exon 1 (designed by Eli Mrkusich). The template for the PCR was a linearised mammalian expression vector containing the MOR-1 gene (courtesy of Bronwyn Kivell). The PCR product was $287 \mathrm{bp}$ in length, as one primary aim of this project was to use small riboprobes (< $100 \mathrm{nt}$ ) for FISH, the PCR product was subjected to restriction digestion to produce smaller fragments for riboprobe synthesis (Figure 3.4).

A restriction map was made for the 287 bp fragment using MapDraw ${ }^{\mathrm{TM}}$ from Lasergene (DNASTAR Inc.). The 287 bp fragment was cut with two restriction enzymes: Hae III (Boehringer Mannheim) and $P v u$ II (Invitrogen ${ }^{\top \mathrm{M}}$ life technologies) to $_{\text {) }}$ produce a 117 bp T7-tailed fragment and a 237 bp T7-tailed fragment, respectively. The $P v u$ II digested fragment contained the partial sequence of the 5' UTR and exon 1 (Figure 3.4). For ease these riboprobes are named: exon 1 riboprobe (Hae III digested 287 fragment), $P v u I I$ riboprobe (partial 5' UTR + entire exon 1) and the MOR 287 fragment riboprobe (entire 5' UTR + exon 1) throughout the remainder of this thesis.

These riboprobes were trialed briefly before the completion of this project, therefore have been placed last in this section. Amplification of the desired product was achieved by PCR. Deep Vent ${ }_{R}^{\circledR}$ (exo-) DNA Polymerase was used in PCR, for the generation of the ds DNA template required for T7-catalysed riboprobe synthesis. Deep Vent (exo-) has been engineered to eliminate the $3^{\prime}=>5^{\prime}$ exonuclease activity; 
therefore polishing of the PCR product was not required, as non-templated adenosines were not added during PCR.

A.

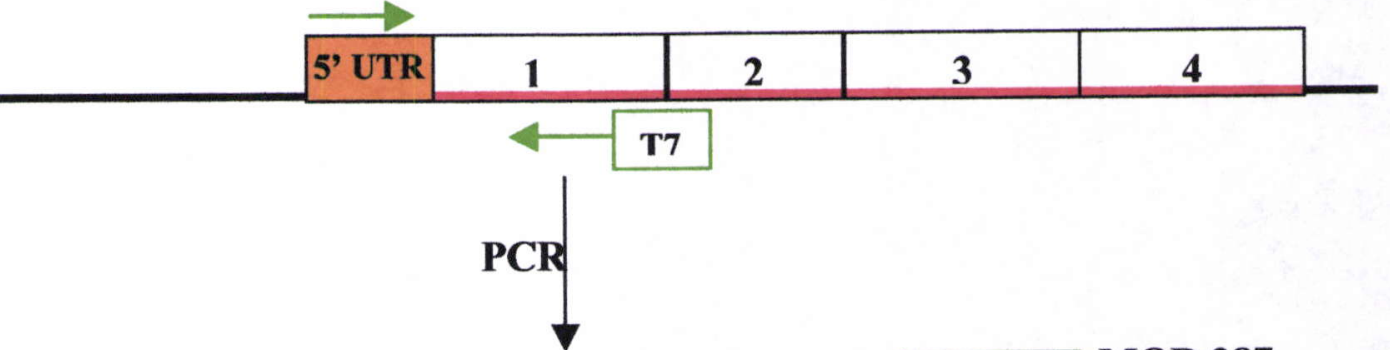

B.

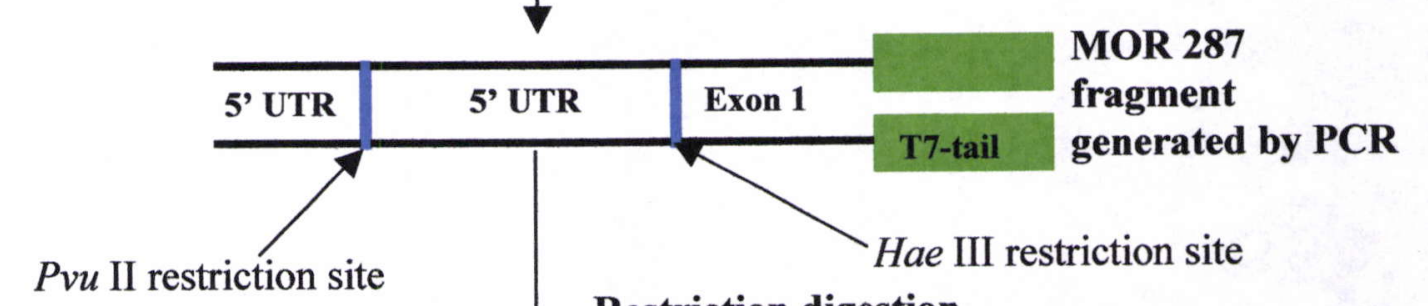

$P v u$ II restriction site

Restriction digestion

C.

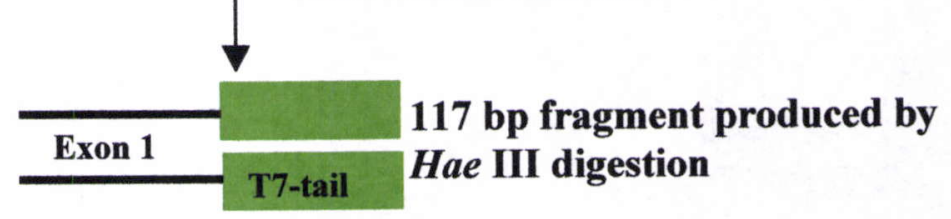

\begin{tabular}{lll}
\multicolumn{2}{c}{ Or } & \\
\cline { 1 - 1 } 5' UTR & & 222 bp fragment produced by \\
\hline
\end{tabular}

D.
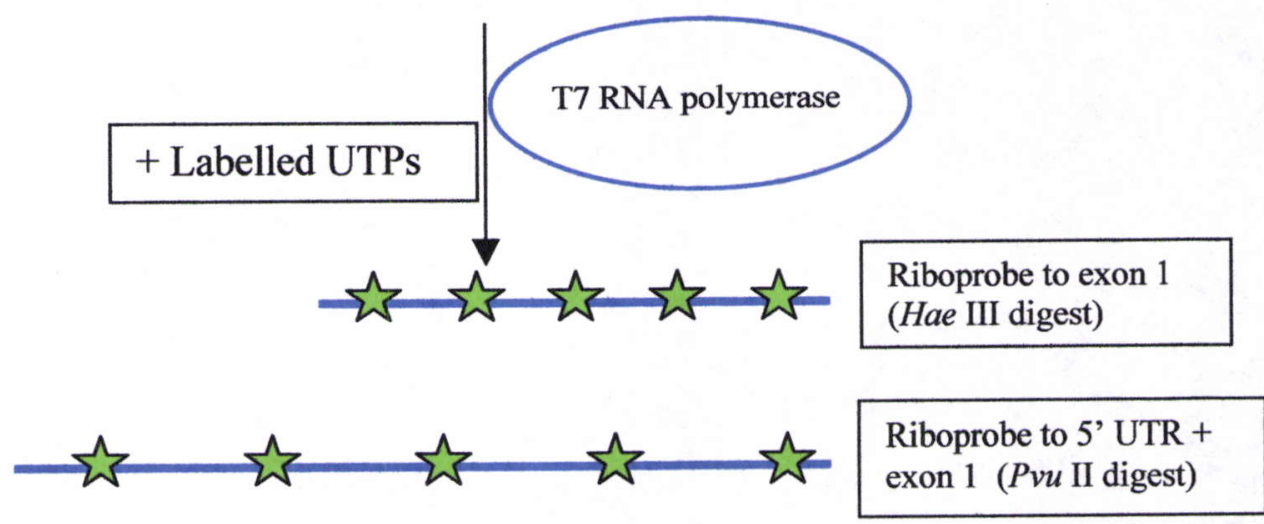

Figure 3.4 Schematic overview of the 5'UTR + exon 1, and exon 1 riboprobe synthesis. A. Depicts a linearised plasmid (black) containing the MOR-1 gene (red). The MOR-1 gene is inserted into the multiple cloning site of the plasmid. The orange box at the beginning of exon 1 represents the 5' untranslated region (UTR). Green arrows represent the primers; the forward primer is complementary to the 5' UTR and the exon 1 reverse primer contains the T7 promoter sequence. B. Shows the MOR 287 fragment, with the restriction sites of Hae III and Pvu II mapped onto the PCR product. C. Represents the two different sized products generated by restriction digestion and riboprobe synthesis initiated by using T7 RNA polymerase (labelled-UTPs $\sum^{3}$ ) D. Depicts the Hae III generated exon 1 riboprobe and the Pvu II generated riboprobe to exon $1+5$ ' UTR. Not drawn to scale. NB The MOR 287 riboprobe is generated by T7 RNA transcription of the MOR 287 fragment before restriction digestion. 


\subsection{Riboprobe Purification}

Purification of the small ds DNA PCR products for riboprobe synthesis and purification of the small riboprobes themselves represented a unique challenge in this project. Purification of both these products was essential for removing any unincorporated nucleotides. Typically PCR products are purified using: gel extraction kits (e.g. CONCERT TM Rapid Gel Extraction System), PCR purification kits (e.g. CONCERT ${ }^{\mathrm{TM}}$ Rapid PCR Purification System), or sephadex spin columns (e.g. Sigma Aldrich ${ }^{\circledR}$ Sephadex G-25 Spin Columns).

However, the small size of the PCR products (73 bp - $95 \mathrm{bp}$ ) and riboprobes ( $73 \mathrm{nt}-$ $95 \mathrm{nt}$ ) resulted in a dramatic loss of product by using any of the aforementioned purification techniques. This is because the spin cartridges provided in purification kits contain silica-based membranes that are unable to absorb short nucleic acid sequences (i.e. $<100 \mathrm{bp}$ ), resulting in a complete or partial loss of these products. Sephadex G-25 Spin Columns used in this project should have been able to purify these small products, but for unknown reasons this technique was met with large losses in product amount. This may have been due to RNase contamination or incorrect calibration of the spin columns (personal communication Dr Darren Day).

In this study, the most successful technique applied to purify PCR products and riboprobes was ethanol precipitation with linear polyacrylamide as a neutral carrier (Gaillard and Strauss 1990) (refer Experimental Procedures). Linear polyacrylamide is an inert substance that acts as a co-precipitant to improve yields of nucleic acids in the precipitation step of purification protocols. $3 \mathrm{M}$ Sodium Acetate $(\mathrm{NaOAc})$ was 
the counter ion used to purify these products by ethanol precipitation; this technique resulted in adequate purification and recovery of each product.

\subsection{Results and Discussion}

\subsubsection{Riboprobe synthesis}

As discussed in section 3.2 Experimental Design all riboprobe templates in this study were generated by PCR using a T7-tailed reverse primer. This PCR product was purified and transcribed to cRNA (riboprobe) using T7 RNA polymerase. As the technique for riboprobe synthesis was analogous for each of the riboprobes, only the synthesis of the exon 4, exon 1, MOR 287 fragment and $P v u I I$ riboprobes are represented in this section.

\section{Exon 4 riboprobe synthesis}

The template for exon 4 riboprobe synthesis was generated by PCR, an aliquot of the product was run on a $2.5 \%$ agarose gel to check that the correctly sized template had been generated (Figure 3.5). The purified PCR product was used as template in a second round PCR to generate enough product for riboprobe synthesis. 


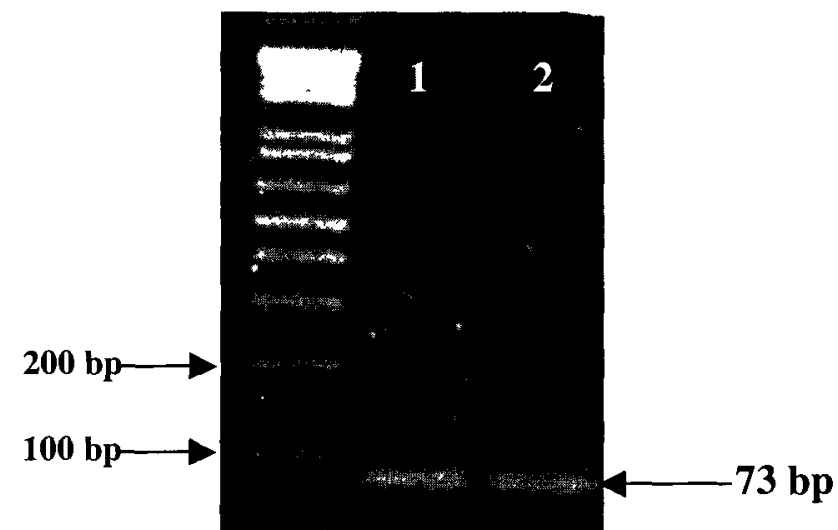

Figure 3.5 Synthesis of the T7-tailed exon 4 riboprobe template by PCR. The PCR was set up with $200 \mathrm{nM}$ each of the MOR exon 4 overlapping oligos (refer section 3.2.1). The reverse oligo was T7-tailed to allow the incorporation of the T7 promoter sequence into the ds DNA template during PCR. The $1 \mathrm{~Kb}^{+}$ladder is loaded in the left lane to allow for size determination. Lane 1 had an annealing temperature of $55^{\circ} \mathrm{C}$ and lane 2 had an annealing temperature of $60^{\circ} \mathrm{C}$. Analysis of PCR products was performed by agarose gel electrophoresis using a $2.5 \%$ agarose gel. Visualisation was achieved using a UV transilluminator after staining the product with $320 \mathrm{ng} / \mathrm{mL}$ ethidium bromide.

Exon 4 riboprobes were synthesised by T7-RNA transcription of the purified PCR product represented in Figure 3.5, this reaction was DNase I treated to digest any remaining DNA template and an aliquot of this reaction was run to check riboprobe synthesis (Figure 3.6).

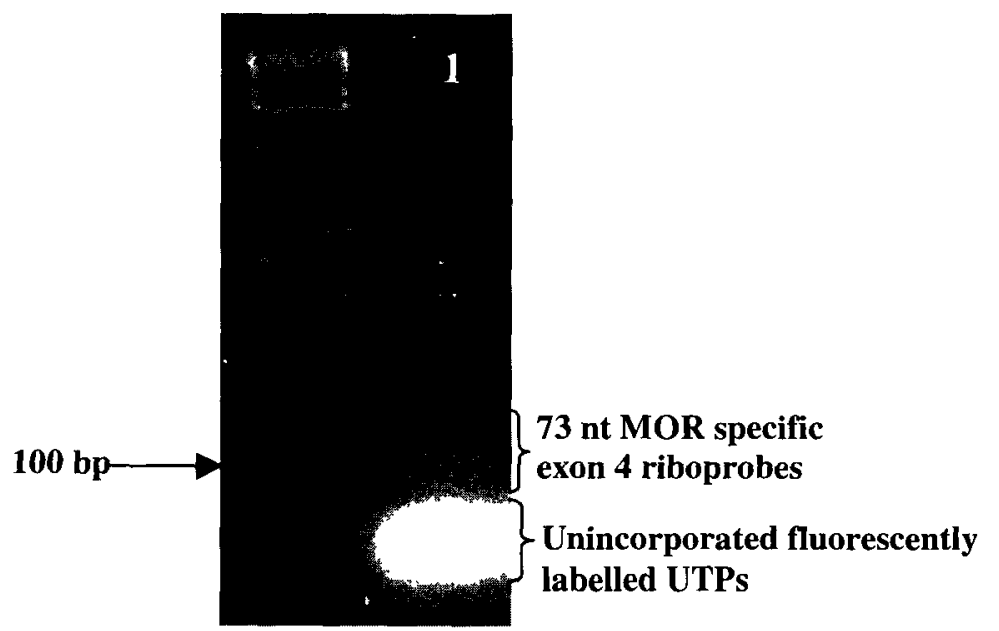

Figure 3.6 In vivo T7-RNA transcription of exon 4 riboprobes. The $1 \mathrm{~Kb}^{+}$ ladder is loaded in the left lane to allow for size determination. Lane 1 shows a "smear" that represents the labelled exon 4 riboprobes; the smeared appearance of the band is most likely due to the riboprobes running with altered mobility due to the varying conformations of non-denatured cRNA. The unincorporated FITC-labelled UTPs can be seen as a bright band below the exon 4 riboprobe. Analysis of riboprobes was performed by agarose gel electrophoresis using a $2.5 \%$ agarose gel. Visualisation was achieved using a UV transilluminator after staining the product with $320 \mathrm{ng} / \mathrm{mL}$ ethidium bromide. 


\section{7 fragment riboprobe synthesis}

The 287 bp fragment was generated by PCR using a forward primer designed to the

5' UTR and a T7-tailed reverse primer designed to a region of exon 1 (Figure 3.7).

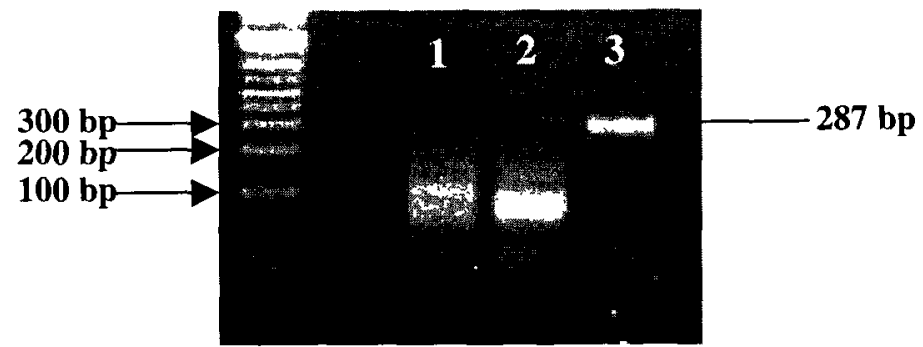

Figure 3.7 Synthesis of the MOR 287 fragment by PCR, including unlabelled exon 4 and exon 9 riboprobes. The $1 \mathrm{~Kb}^{+}$ladder is loaded in the left lane to allow for size determination. Lanes 1 and 2 show the synthesis of unlabelled exon 4 and 9 riboprobes, respectively. These samples were not heat denatured and the different conformations of the riboprobe are visible as two distinct bands. Lane 3 represents the 287 bp PCR product used to generate the exon 1 and Pvu II riboprobes. Analysis of products was performed by agarose gel electrophoresis using a $2.5 \%$ agarose gel. Visualisation was achieved using a UV transilluminator after staining the product with $320 \mathrm{ng} / \mathrm{mL}$ ethidium bromide.

The $287 \mathrm{bp}$ fragment spans from the 5' UTR into exon 1 of the Oprm. Digesting the 287 fragment with Hae III generated the 117 bp exon 1 riboprobe template (Figure 3.8). The $P v u I I$ riboprobe (Figure 3.8) was generated by restriction digestion of the 287 fragment with $P v u I I$.

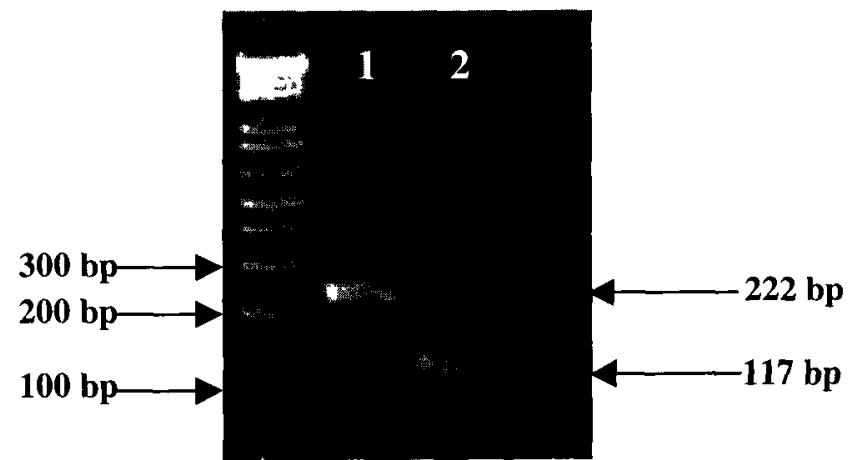

Figure 3.8 Restriction digestion of the MOR 287 bp fragment to generate the exon 1 and Pvu I/ riboprobe templates. The $1 \mathrm{~Kb}^{+}$ladder is loaded in the left lane to allow for size determination. Lane 1 represents the Pvu // digest, two bands can be seen the T7-tailed 222 bp fragment and a 75 bp fragment. Lane 2 represents the Hae III digest, with two bands running at approximately the same size: a T7-tailed $117 \mathrm{bp}$ fragment and a $170 \mathrm{bp}$ fragment. Analysis of the digested products was performed by agarose gel electrophoresis using a $2.5 \%$ agarose gel. Visualisation was achieved using a UV transilluminator after staining the product with $320 \mathrm{ng} / \mathrm{mL}$ ethidium bromide. 
After restriction digestion the enzymes were heat inactivated and the reactions were purified and used as template in the generation of riboprobes. The riboprobe synthesis reactions were run on a $2.5 \%$ agarose gel to check transcription efficiency (Figure 3.9).

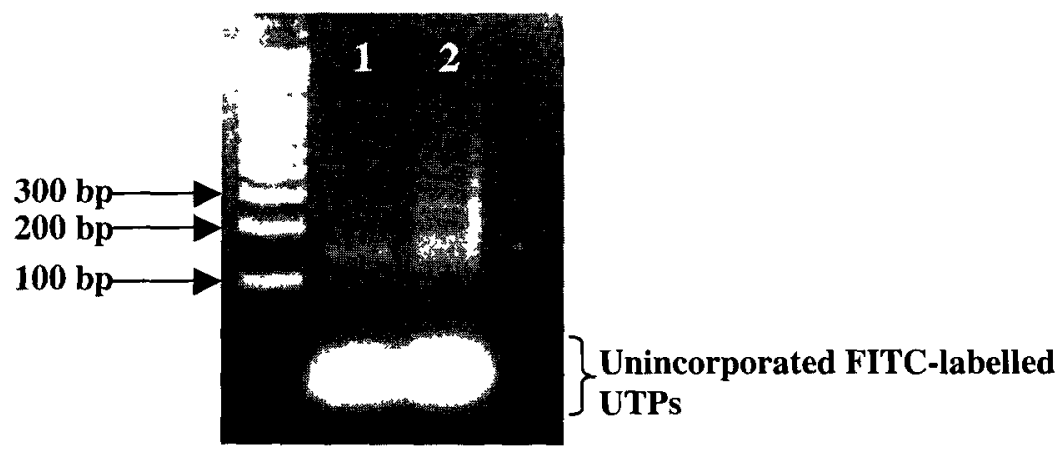

Figure 3.9 Labelled riboprobes synthesised to exon 1 and the 5' UTR + exon 1 ( $P v u$ II digest). The $1 \mathrm{~Kb}^{+}$ladder is loaded in the left lane to allow for size determination. Lane 1 shows the $117 \mathrm{nt}$ exon 1 riboprobes. Lane 2 represents the 222 nt $P v u$ // riboprobes. Two bands are visible in each lane due to the alternative conformations of cRNA. Analysis of the riboprobes was performed by agarose gel electrophoresis using a $2.5 \%$ agarose gel. Visualisation was achieved using a UV transilluminator after staining the product with $320 \mathrm{ng} / \mathrm{mL}$ ethidium bromide.

\subsubsection{Analysis of synthesised riboprobes}

Newly synthesised riboprobes were analysed for size, sequence, yield, integrity and quality before being used in FISH experiments. Two methods were used to determine the actual size of the riboprobe. The first method is an estimation of size based on agarose gel electrophoresis (see Figure 3.9), however as mentioned, riboprobes tend to run in two conformations unless heat denatured.

The second, more accurate method is to perform a GeneScan analysis of a diluted aliquot of the riboprobe (refer Experimental Procedures, 4.16.2). Due to the small 
size of the riboprobes in this project a GeneScan ${ }^{\mathrm{TM}}-350$ ROX size standard (Applied Biosystems) was used to determine the actual size of the riboprobe (Appendix A).

Samples of each riboprobe were analysed on the ABI PRISM ${ }^{\circledR} 377$ DNA sequencer. These samples were heated and then snap chilled before being loaded onto the gel to ensure the cRNA remained single stranded. The results generated were analysed using GeneScan ${ }^{\circledR}$ Analysis Software and riboprobe size was determined by comparison to the ROX-350 size standard loaded with each sample (Figure 3.10).

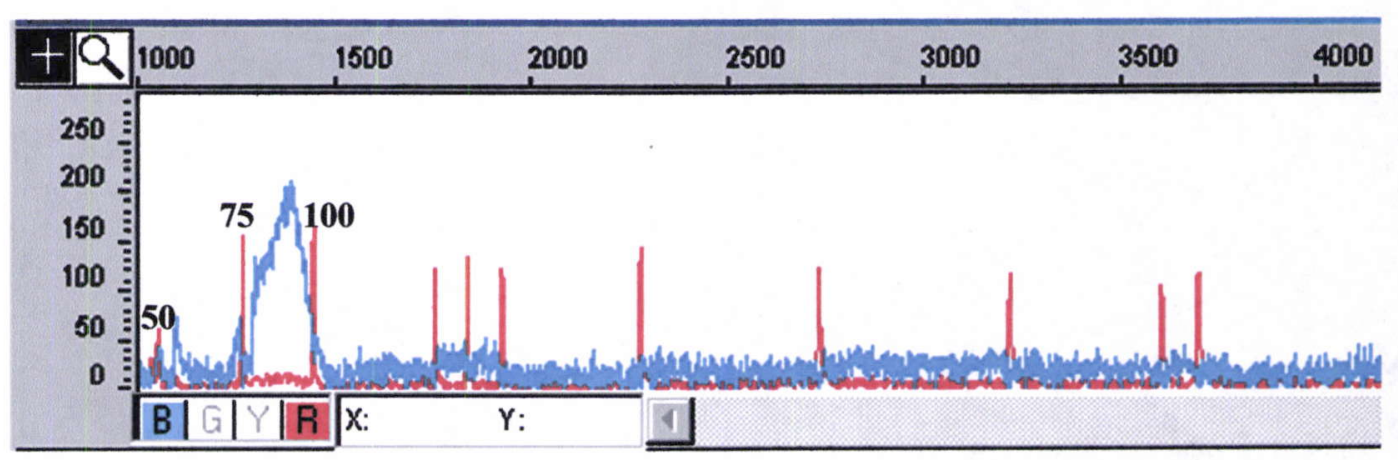

Figure 3.10 Size determination of a FITC-labelled exon 9 riboprobe. The exon 9 riboprobe was diluted 1000 -fold before being loaded onto the $4 \%$ PAGE. This histogram was generated using GeneScan Analysis Software version 3.7. The size of the riboprobe is determined by comparison to the GeneScan ${ }^{\mathrm{TM}}-350 \mathrm{ROX}$ size standard represented by red peaks. The large blue peak represents the exon 9 riboprobe with an expected size of $95 \mathrm{bp}$.

GeneScan analysis of riboprobes is an excellent tool, not only for size calling but also for determining the yield and quality of riboprobe synthesis. Another important experiment to perform to check riboprobe synthesis is to sequence the ds DNA template used for cRNA transcription (refer Experimental Procedures, 4.16.1). 
Riboprobes were sequenced to ensure they were designed and made to target specific regions of the Oprm (refer Appendix B, for the chromatogram produced by sequencing the ds DNA template used to synthesise exon 3 riboprobes). Approximately only $68 \mathrm{nt}$ out of $100 \mathrm{nt}$ (including the forward and reverse sequences) were able to be base called for exon 3 and the resulting sequence was compared to sequences on the NCBI database using BLAST.

BLAST results showed that the exon 3 riboprobes made in this project shared a $93 \%$ identity with Mus musculus MOR mRNA (Appendix Figure B3). Whereas approximately $61 \mathrm{nt}$ out of $73 \mathrm{nt}$ of the exon 4 riboprobe were able to be base called with confidence (Appendix, Figure B4) and the BLAST results showed that the exon 4 riboprobe template shared a $100 \%$ identity with the Mus musculus MOR exon 4 and MOR-1G mRNA (Figure 3.11).

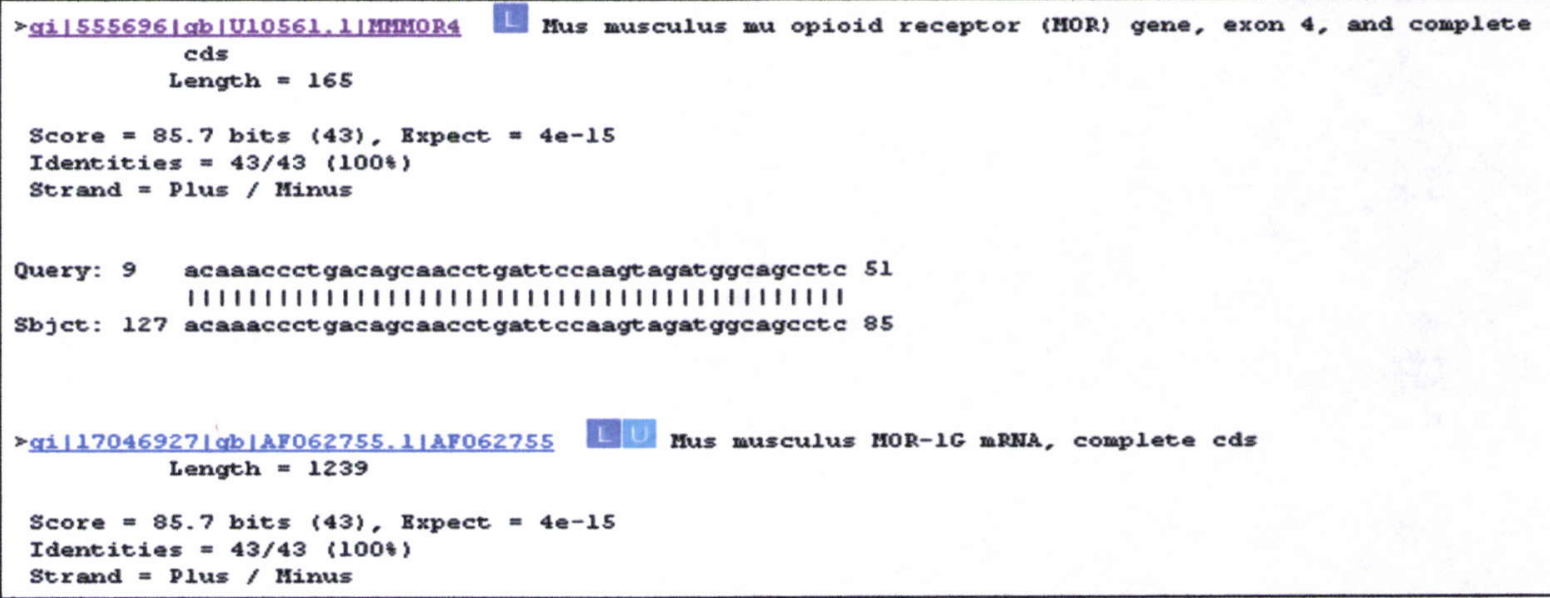

Figure 3.11 Top two BLAST search results using exon 4 sequence (refer Appendix Figure B4). These results show that there is $100 \%$ identity of the exon 4 riboprobe template sequence to the Mus musculus exon 4 gene and the MOR-1G splice variant that contains exon 4. 
The yield of each riboprobe was determined by performing a SYBR Green II assay (refer Experimental Procedures 4.16.2). Denaturing PAGE and agarose gel electrophoresis confirmed the integrity and quality of the riboprobes. If a distinct bright band was present at the expected size it was presumed that the riboprobe was not degraded. The integrity of each "batch" of riboprobe was checked before the commencement of FISH experiments.

\subsubsection{Interpreting FISH Results}

This section presents the results and discussion of FISH experiments using riboprobes designed to exon 3, 4, 9, 1, including the $P v u I I$ riboprobe and the MOR 287 fragment. Initially many FISH experiments were performed in order to find the most stringent conditions under which the small riboprobes in this project would hybridise (refer Section 4.18).

When examining FISH results, certain factors have to be taken into account before one can draw the conclusion that the signal being visualised represents specific hybridisation of the riboprobe to its cognate mRNA target.

High background signals and quenching are two phenomena that can hinder image analysis. As riboprobes designed to different splice variants were utilised in this project to map splice variant distribution, they had to be differentially labelled. The chance of high background signals and quenching increased due to the use of two

different fluorophores, therefore prior to riboprobe synthesis certain factors to decrease these phenomena, were taken into account. It was assumed from the specific activity of each riboprobe and the labelling ratio used that fluorescently 
labelled UTPs were incorporated into the riboprobe approximately $10 \mathrm{nts}$ apart to prevent quenching and high background signals. Also the two fluorophores used in this project (CY3-UTPs and fluoroscein-12-UTPs) were chosen for double labelling experiments due to the spectral separation of the two fluorescent emissions $(560 \mathrm{~nm}$ and $495 \mathrm{~nm}$ respectively). Maximising the spectral separation of multiple fluorescent emissions facilitates signal isolation and image analysis.

Stringent positive controls must be performed simultaneously with each FISH to aid the interpretation of each result. One important positive control experiment is the design and use of sense strand riboprobes. Sense riboprobes are the same sequence as the target mRNA. A common method for in vitro RNA synthesis is the use of cloning vectors. Multipurpose cloning vectors contain a multiple cloning site (MCS) into which the gene of interest is inserted. The MCS is flanked on either side by promoter sequences for different polymerases (e.g. T7 promoter sequence required for T7 RNA polymerase transcription). This allows the synthesis of either sense or antisense riboprobes from the sequence cloned into the MCS.

Sense riboprobes should not remain bound after stringent posthybridisation washes are applied. However this experiment does have limitations, because any nucleic acids added to FISH will bind non-specifically and it is possible that a sense riboprobe could hybridise to a GC-rich nucleic acid sequences and remain bound after the posthybridisation washes.

One important limitation of this study is that sense riboprobes were not generated for any of the riboprobes used, due to time constraints. This must be taken into account 
when analysing the images presented in this project. One must also consider the issue of tissue autofluorescence; this can severely compromise the fluorescent detection sensitivity by increasing background signals. Autofluorescence may originate from endogenous sample constituents such as lipofuscin. Lipofuscin is a fluorescent pigment that accumulates with age in the cytoplasm of CNS cells.

To determine whether the signals visualised in this project were due to autofluorescence images were captured under a different narrow band filter set, to that of the fluorophore the riboprobe was labelled with. For example images of a CY3-labelled riboprobe would be captured under the CY3 filter set and the FITC filter set. As tissue autofluorescence typically exhibits broad excitation and emission spectra, one would expect to see the same signal intensities and localisations under both filter sets if it were autofluorescence (Schnell et al. 1999).

Another consideration is signal intensity, all the images in this project were analysed using Adobe Photoshop version 5.5 to determine the intensity of the signal over the background. These data are represented in Table 3.2, where the difference in the median values between the signal and the background has been converted to a percentage that represents how much greater than the background the signal for each FISH experiment was. These percentages (Table 3.2) represent a value of confidence, whereby signals greater than $40 \%$ over the background infer one can be confident that the signal visualised represents fluorescence detection above background fluorescence and autofluorescence. The raw data can be located in Appendix E, with a sample calculation. 


\begin{tabular}{|c|c|c|c|}
\hline Figure & Riboprobe & $\begin{array}{l}\text { Percentage signal is } \\
\text { above background (\%, } \\
2 \text { s.d.) }\end{array}$ & Confidence in signal \\
\hline 3.14 & CY3-Ex3 & 20.39 & $\checkmark$ \\
\hline $3.15, \mathrm{~A}$ & FITC-Ex4 & $\begin{aligned} \mathrm{ML} & =55.13 \\
\mathrm{GL} & =24.36\end{aligned}$ & $\begin{array}{l}\checkmark \checkmark \checkmark \\
\checkmark \checkmark\end{array}$ \\
\hline $3.15, \mathrm{C}$ & FITC-Ex4 & 40.85 & $\sqrt{ } \sqrt{ }$ \\
\hline $3.15, \mathrm{E}$ & FITC-Ex4 & 54.26 & $\checkmark \checkmark \checkmark$ \\
\hline $3.16, \mathrm{~A}$ & FITC-Ex 9 & 26.92 & $\checkmark \checkmark$ \\
\hline $3.16, \mathrm{C}$ & CY3-Ex9 & 39.40 & $\checkmark \checkmark \checkmark$ \\
\hline $3.17, \mathrm{~A}$ & FITC-Ex1 & $\begin{aligned} \mathrm{ML} & =52.44 \\
\mathrm{GL} & =12.20\end{aligned}$ & $\begin{array}{l}\checkmark \checkmark \checkmark \\
\checkmark\end{array}$ \\
\hline $3.17, \mathrm{D}$ & FITC-Ex 1 & 47.62 & $\checkmark \checkmark \checkmark$ \\
\hline $3.18, \mathrm{~A}$ & FITC-Pvu2 & 76.06 & $\checkmark \checkmark \checkmark$ \\
\hline $3.18, \mathrm{C}$ & FITC-Pvu2 & 76.83 & $\checkmark \checkmark \checkmark$ \\
\hline $3.18, \mathrm{E}$ & FITC-Pvu2 & $\begin{aligned} \mathrm{ML} & =66.67 \\
\mathrm{GL} & =42.53\end{aligned}$ & $\begin{array}{l}\checkmark \checkmark \checkmark \\
\checkmark \checkmark \checkmark\end{array}$ \\
\hline $3.19 \mathrm{a}, \mathrm{A}$ & FITC-Ex 1 & $\begin{aligned} \mathrm{ML} & =56.60 \\
\mathrm{GL} & =22.33\end{aligned}$ & $\begin{array}{l}\checkmark \checkmark \checkmark \\
\checkmark \checkmark\end{array}$ \\
\hline $3.19 \mathrm{a}, \mathrm{B}$ & CY3-Ex4 & $\begin{array}{l}\mathrm{ML}=36.29 \\
\mathrm{GL}=20.16\end{array}$ & $\begin{array}{l}\checkmark \checkmark \\
\checkmark\end{array}$ \\
\hline $3.19 \mathrm{a}, \mathrm{D}$ & FITC-Pvu2 & $\begin{aligned} \mathrm{ML} & =68.33 \\
\mathrm{GL} & =39.17\end{aligned}$ & $\begin{array}{l}\checkmark \checkmark \checkmark \\
\checkmark \checkmark\end{array}$ \\
\hline $3.19 \mathrm{a}, \mathrm{E}$ & CY3-Ex4 & $\begin{array}{l}\mathrm{ML}=30.00 \\
\mathrm{GL}=14.00\end{array}$ & $\begin{array}{l}\checkmark \checkmark \\
\checkmark\end{array}$ \\
\hline $3.19 \mathrm{~b}, \mathrm{~A}$ & FITC-287 & $\begin{aligned} \mathrm{ML} & =58.02 \\
\mathrm{GL} & =28.40\end{aligned}$ & $\begin{array}{l}\checkmark \checkmark \checkmark \\
\checkmark \checkmark\end{array}$ \\
\hline $3.19 b, B$ & CY3-Ex4 & $\begin{aligned} \mathrm{ML} & =31.36 \\
\mathrm{GL} & =10.17\end{aligned}$ & $\begin{array}{l}\checkmark \checkmark \\
\checkmark\end{array}$ \\
\hline $3.19 b, D$ & FITC-287 & $\begin{aligned} \mathrm{ML} & =54.67 \\
\mathrm{GL} & =28.00\end{aligned}$ & $\begin{array}{l}\checkmark \checkmark \checkmark \\
\checkmark \checkmark\end{array}$ \\
\hline $3.19 b, E$ & CY3-Ex4 & $\begin{aligned} \mathrm{ML} & =31.20 \\
\mathrm{GL} & =20.00\end{aligned}$ & $\begin{array}{l}\checkmark \checkmark \checkmark \\
\checkmark\end{array}$ \\
\hline $3.20 \mathrm{a}, \mathrm{A}$ & FITC-Ex1 & 58.59 & $\checkmark \checkmark \checkmark$ \\
\hline $3.20 \mathrm{a}, \mathrm{B}$ & CY3-Ex4 & 46.15 & $\checkmark \checkmark \checkmark$ \\
\hline $3.20 \mathrm{a}, \mathrm{D}$ & FITC-Pvu2 & $69 . \overline{44}$ & $\checkmark \checkmark \checkmark$ \\
\hline $3.20 \mathrm{a}, \mathrm{E}$ & CY3-Ex4 & 23.14 & $\checkmark \checkmark$ \\
\hline $3.20 \mathrm{~b}, \mathrm{~A}$ & FITC-287 & 62.35 & $\checkmark \checkmark \checkmark$ \\
\hline $3.20 \mathrm{~b}, \mathrm{~B}$ & CY3-Ex4 & 34.13 & $\checkmark \checkmark$ \\
\hline $3.21, \mathrm{~A}$ & FITC-ExI & 29.73 & $\sqrt{ } \checkmark$ \\
\hline $3.21, \mathrm{~B}$ & CY3-Ex4 & 32.14 & $\checkmark \checkmark$ \\
\hline $3.21, \mathrm{D}$ & FITC-287 & 65.83 & $\checkmark \checkmark \checkmark$ \\
\hline $3.21, \mathrm{E}$ & CY3-Ex4 & 49.60 & $\checkmark \checkmark \checkmark$ \\
\hline
\end{tabular}

Table 3.2 Table of confidence for signal intensities. The above table gives the percentage value for fluorescent detection over the background fluorescence and autofluorescence. $\quad \checkmark \checkmark \checkmark \quad(>40 \%)=$ confident signal represents fluorescent detection, $\checkmark \checkmark(20-40 \%)=$ probable signal represents fluorescent detection, $\checkmark$ (0$20 \%$ ) $=$ unable to determine is signal represents fluorescent detection as signal is too close to the background value. NB. $\mathrm{ML}=$ molecular layer of the cerebellum, $\mathrm{GL}$ = granular layer of the cerebellum, where the density of neurons is very high, therefore increasing the intensity of the background relative to the signal. 
Structures were identified with reference to a rat brain atlas (Kruger et al. 1995) to determine where staining was visualised. Figure 3.12 outlines the important structures referred to in this project.
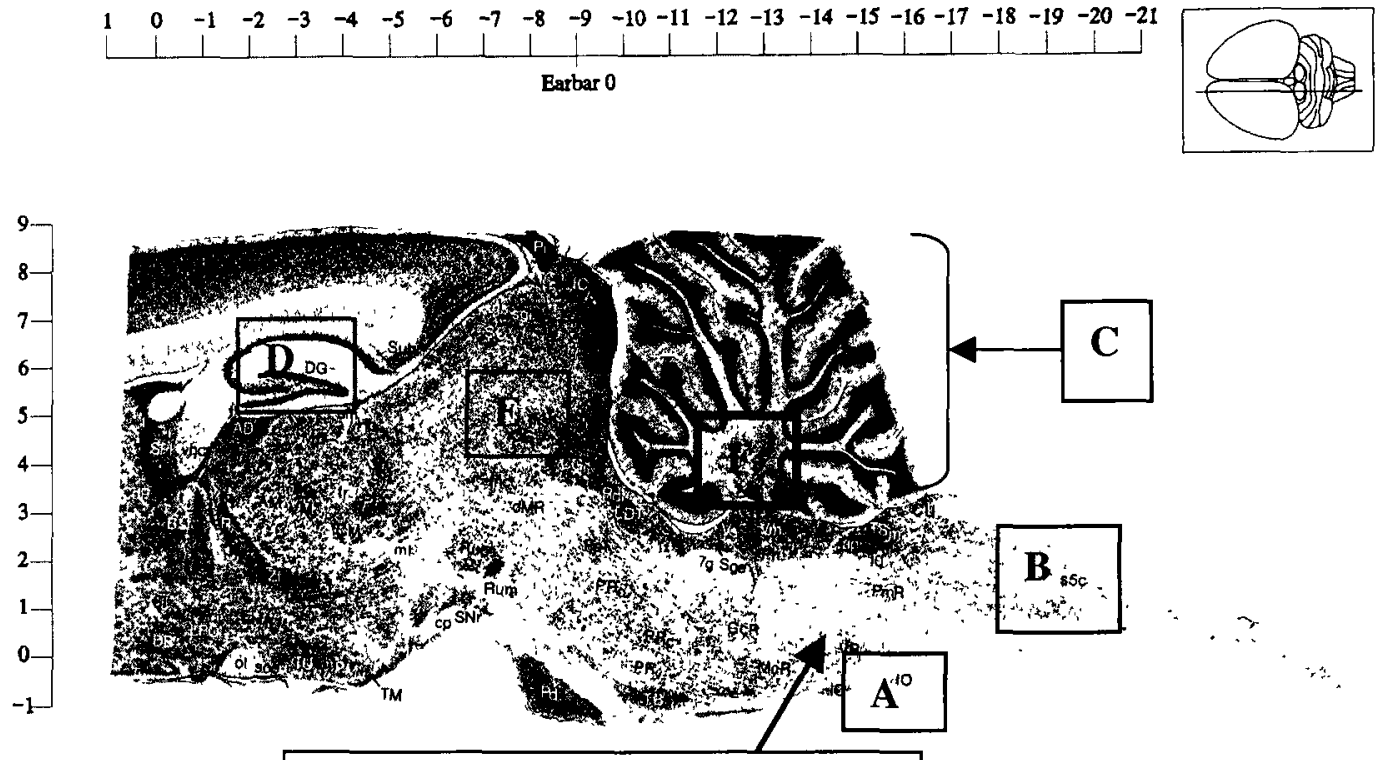

Location of nucleus ambiguus in sagittal section 2.4

Sagittal 1.2

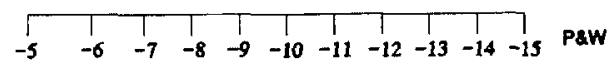

Figure 3.12 Sagittal section 1.2 of adult rat brain near midline. The major structures of the brain discussed in this thesis are highlighted above. A. Inferior olive. B. Spinal trigeminal nucleus. C. Cerebellum. D. Dendate gyrus. E. Central nucleus inferior colliculus. F. Interposed (intermediate or interpositus) cerebellar nucleus. The arrow indicates area the nucleus ambiguus is located in sagittal section 2.4. Image taken from Photographic Atlas of the Rat Brain, Plate 106 (Kruger et al. 1995).

\subsubsection{Image Adjustment}

All images were adjusted using Adobe Photoshop version 5.5 software, Figure 3.12 represents an adjusted and an unadjusted image and the histograms for each image before and after the manipulation. Typically, the grey scale levels were adjusted to enhance the signal of the fluorophore of interest, e.g. CY3 riboprobe images were maximised for red and the output levels for blue and green were reduced to zero. 
Figure 3.13 exemplifies the adjustments made to images in this project and also shows how a fluorescent signal can be optimised (Figure 3.13, panels C and D).
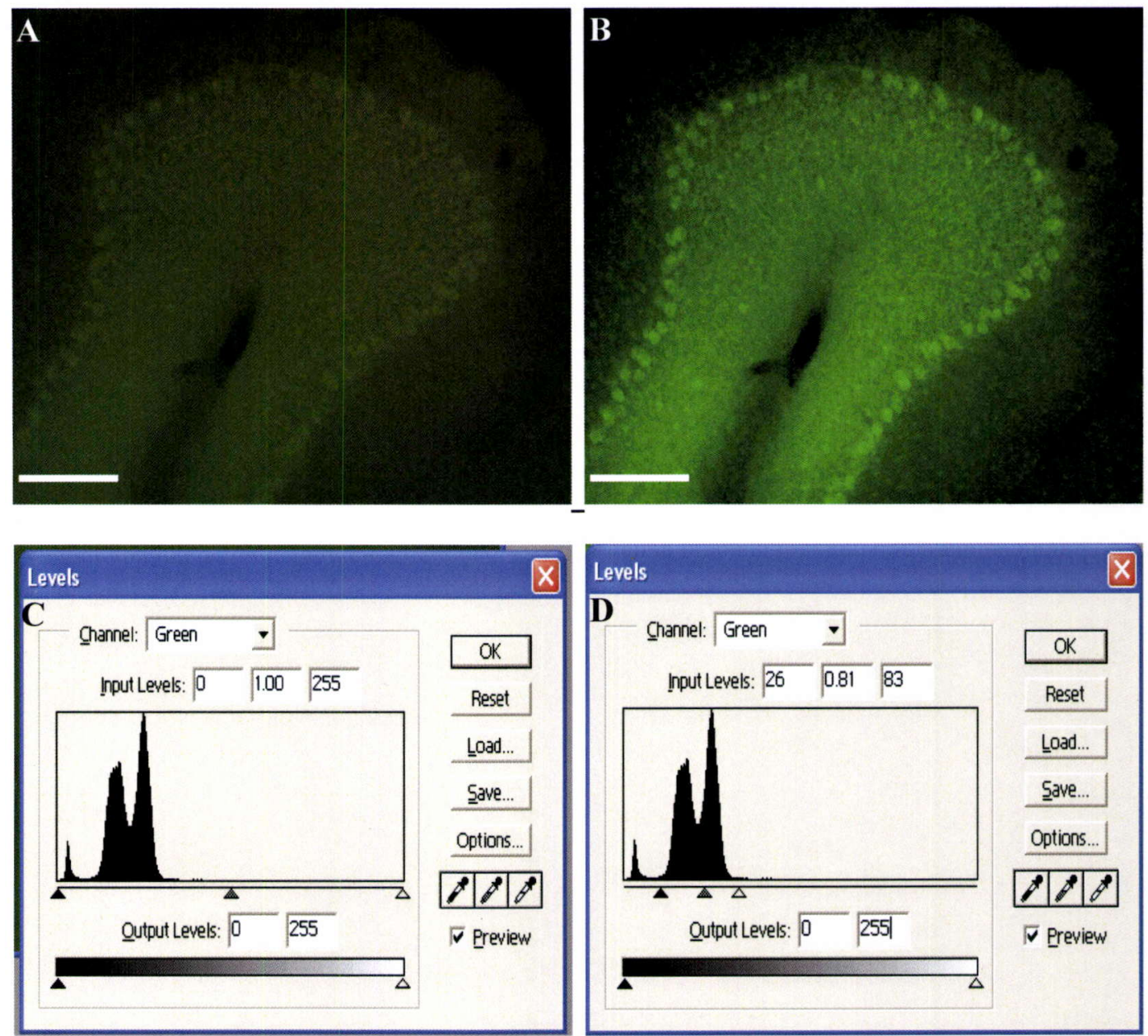

Figure 3.13 Image manipulation. Panels $\mathrm{A}$ and $\mathrm{B}$ are the same image of an exon 4 FITC-labelled riboprobe hybridising to the Purkinje cells in the cerebellum. Panels $C$ and $D$ represent the levels of green for images $A$ and $B$ respectively. Before the image is adjusted the input and output grey levels were 0-255 and after manipulation the input and output grey levels were 26-83, respectively. Scale bar $=$ $200 \mu \mathrm{m}$.

\subsubsection{Exon 3 riboprobe}

Exon 3 riboprobes showed staining in the middle cerebellar peduncle (Figure $3.14 \mathrm{~A}$ and B). The hybridisation of this probe only to the middle cerebellar peduncle, suggests that the exon 3 riboprobe was not binding specifically, as exon 3 is present 
in all 15 of the MOR splice variants. It would be expected that the exon 3 riboprobe would hybridise to all brain structures known to express MOR.
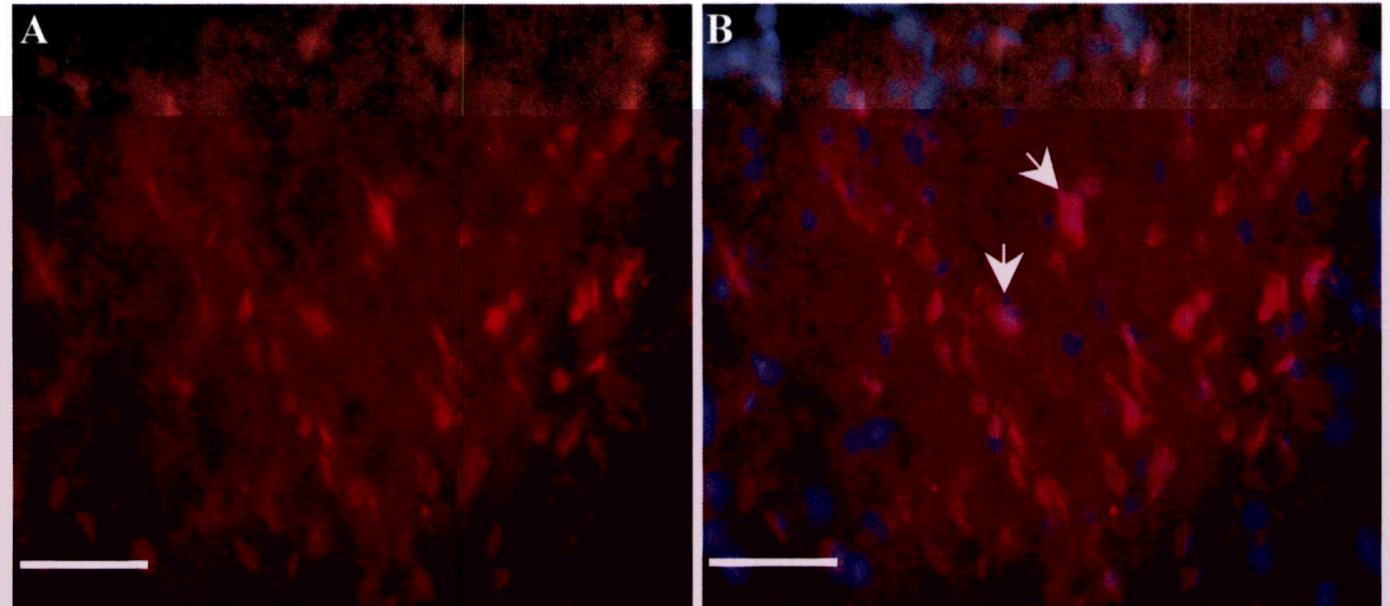

Figure 3.14 CY3-labelled exon 3 riboprobes. Fluorescent photomicrographs of a sagittal section through the middle cerebellar peduncle (A and $B$ ). The exon 3 riboprobe appears to have localised to axonal processes $(\mathbf{A})$. The arrows in panel $\mathbf{B}$ represent regions where the exon 3 riboprobe has localised to axonal processes containing a nucleus (blue DAPI stain). Scale bar $=50 \mu \mathrm{m}$ (A and $\mathbf{B})$.

Figure 3.14 shows that the exon 3 riboprobe clearly has not stained message in the cytoplasm of the neurons in the middle cerebellar peduncle, as no cytoplasmic staining is visible (panel A). It appears that the exon 3 riboprobes have stained axonal processes, because when the image is overlaid with the DAPI nuclear stain (panel B), only some areas of hybridisation show the presence of a central nucleus (represented by arrows in panel B).

Many different experimental conditions (such as altered hybridisation temperatures and stringencies) were trialed for the exon 3 riboprobe and Figure 3.14 represents the only FISH experiment to have produced staining. However, this result could not be duplicated in any further experiments with the same riboprobe, suggesting that the results shown in Figure 3.14 are artifactual. 
As mentioned, due to the fact that the exon 3 riboprobes did not hybridise to other structures of the brain known to express MOR, and as rigorous positive and negative controls were not performed simultaneously with these FISH, it is likely that the staining seen in Figure 3.14 does not represent specific localisation of the exon 3 riboprobe to mRNA. The exon 3 riboprobe was sequenced and was shown to share 93\% identity (68 nt out of $100 \mathrm{nt}$ ) with the mu-opioid receptor (refer Appendix B), suggesting that the riboprobe was correctly designed.

The possible reasons for the lack of significant and reproducible results using the riboprobe designed to exon 3 are numerous. It is possible that the preparation or use of the exon 3 riboprobes in FISH experiments resulted in the degradation of the cRNA by RNase contamination. cRNA degradation would cause the absence of signal experienced in the majority of experiments using exon 3 riboprobes.

It is also possible that the exon 3 riboprobe was designed to a region of the Oprm mRNA that is difficult to access. To examine the accessibility of the target region a computer programme such as mfold (http://www.bioinfo.rpi.edu/ zukerm/rna/) could be used, this programme is also able to predict stable secondary structure conformations of DNA or RNA sequences.

Conceivably the incorrect sequence may have been amplified by PCR. The $100 \mathrm{bp}$ ds DNA template was sequenced, however due to the small size of the template, only $50-70 \%$ of each sequence could be base called with any confidence. It is certain that the correct exon 3 primers were incorporated, as they can be located in the chromatogram of the exon 3 sequence (Appendix B) but the primers may have 
misprimed off the template and incorporated a sequence other than that of exon 3 . If this were so, then only $40 \mathrm{nts}$ of the $100 \mathrm{nt}$ riboprobe would be specific for exon 3 (incorporated primer sequence), this would result in the riboprobe not hybridising to its target under the stringent conditions used in these FISH experiments. Cloning the exon 3 riboprobe would resolve the above issue.

Synthesising an exon 3 riboprobe template using overlapping oligos would guarantee that the sequence was correct (as the oligos are manufactured by reputable companies, such as Invitrogen ${ }^{\top M}$ life technologies), therefore eliminating the possibility of a mispriming event during PCR. This represents a future possibility for the design and synthesis of a riboprobe template for exon 3 of the Oprm.

Due to the difficulty experienced with the exon 3 riboprobe, FISH experiments were performed using the exons 1, 4, Pvu II generated 5'UTR + exon 1 and MOR 287 fragment riboprobes, as these probes appeared to produce more consistent staining.

\subsubsection{Exon 4 riboprobe}

Exon 4 is present in 7 of the 15 MOR splice variants and the riboprobe designed in this project specific to exon 4 was observed to stain the majority of brain structures where high levels of MOR have previously been reported (Abbadie et al. 2000b). The exon 4 riboprobe showed staining in the inferior olive (Figure 3.20a and Figure 3.20b), spinal trigeminal nucleus (Figure 3.21), dendate gyrus (Appendix D), interposed cerebellar nucleus (Figure 3.15, panel E), lateral reticular nucleus (Figure 3.15, panel C) and the cerebellum (Figure 3.15, panel A). 
The localisation of the exon 4 riboprobe to MOR mRNA in the rat cerebellum is a significant result, as prior to recent research completed at Victoria University of Wellington it was generally accepted that DOR and not MOR were expressed in the rat cerebellum (Ableitner 1994, Abeyta et al. 2002). Data presented in this manuscript confirms the presence of MOR mRNA within the Purkinje cells of the adult rat cerebellum (Mrkusich et al. 2003).

Previously ligand-binding studies had provided some evidence for the existence of MORs in the rat cerebellum during development (Barg and Simantov 1989). RTPCR has been utilised to show the presence of MOR mRNA in the rat cerebellum (Wittert et al. 1996), however it is generally accepted that only DOR is expressed in the cerebellum.

The results from this project support the current hypothesis that MOR, and in particular MOR-1 mRNA is present in the rat cerebellum. All exon 4 riboprobes synthesised during this project appear to have hybridised specifically. Control experiments performed demonstrated the specificity of the exon 4 riboprobes to hybridise to MOR mRNA, including an RNase digestion to remove any RNA present in the tissue sections. The FISH executed on RNA digested tissue using an exon 4 riboprobe resulted in the abolition of signal, suggesting that the exon 4 riboprobes used were hybridising to RNA. However, as RNases are notoriously difficult to inactivate, one could also conclude that the RNase was not completely inactivated or removed, therefore resulting in exon 4 riboprobe degradation. 
Further experimentation was carried out in order to determine whether or not the exon 4 riboprobes were hybridising to RNA. A NaOH hydrolysis of the tissue was performed before riboprobe hybridisation, using an exon 4 riboprobe that was known to have localised to specific brain structures that express MORs (Figure 3.15, panel A).

$\mathrm{NaOH}$ hydrolysis of the brain tissue resulted in all RNA being destroyed and unlike RNase digestion, performing multiple rinses using decreasing concentrations of SSC removed all remaining $\mathrm{NaOH}$ (refer Experimental Procedures). Specificity of the exon 4 riboprobe for RNA was confirmed by an absence of signal when performing a FISH on $\mathrm{NaOH}$-treated tissue (refer 3.1, controls, Figure 3.0). This result suggests that under the stringent conditions used for each FISH the exon 4 riboprobe did not exhibit non-specific binding to any other cellular constituents e.g. DNA. The abolition of probe hybridisation signal when using increasingly higher temperatures and more stringent hybridisation washes, also suggests that this riboprobe was specific for its cognate mRNA sequence, but this is not strong evidence that the riboprobe was specific for its target, as all interactions (specific or non-specific) can be washed off under high temperatures and stringencies. Sequencing of the exon 4 riboprobe template showed that it shared a $100 \%$ identity to that of the Oprm exon 4 (Appendix B4). Taken together these data suggest the exon 4 riboprobe was most likely specific for MOR mRNA. 

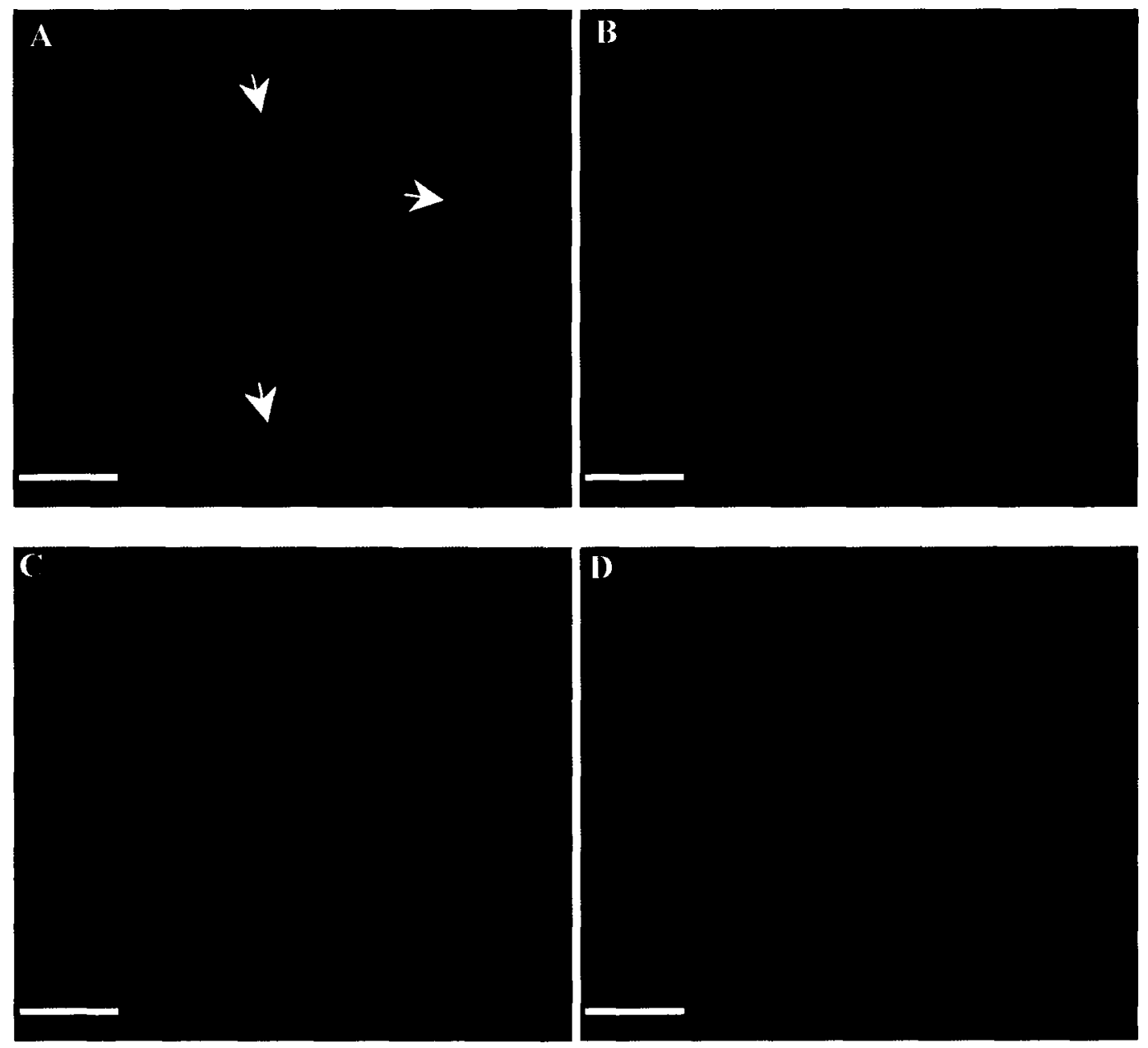

F

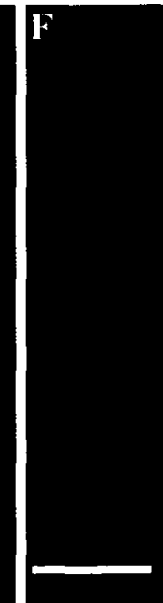

Figure 3.15 FITC-labelled exon 4 riboprobes. Fluorescent photomicrographs of sagittal sections through the cerebellum ( $A$ and $\mathbf{B}$ ), the lateral reticular nucleus ( $C$ and D) and the interposed cerebellar nucleus, including part of the paramedian lobule located in the upper left corner (E and $\mathbf{F}$ ). The white arrows indicate individual neurons within the Purkinje layer stained for Oprm exon 4 mRNA (A and E), some stellate and basket cells can also be seen in the molecular layer of the cerebellum (E). NB The large cluster of neurons to the right-hand side of Panel $\mathbf{E}$ represents the interposed cerebellar nuclei. Scale bar $=200 \mu \mathrm{m}$. 
Figure 3.15 represents selected images exhibiting FITC-labelled exon 4 riboprobe localisation to large cells of the Purkinje layer of the cerebellum (panel A), the lateral reticular nucleus (panel C) and the interposed cerebellar nucleus (panel E). Panels B, D and F represent images of the same structures taken under the CY-3 filter set respectively, these images show that the signal visualised under the FITC filter set is not tissue autofluorescence.

It has been assumed that the riboprobe to exon 4 is detecting mainly the MOR-1 splice variant due to the relative abundance of this splice variant in comparison to the other less known splice variants that contain exon 4 (MOR-1G - MOR-1L). However, it is possible that the exon 4 riboprobes used in this project are detecting these other splice variants.

One experiment would be to design a riboprobe to exon 11 of the Oprm. A FISH utilising exon 11 and exon 4 riboprobes labelled with different fluorophores could be performed, and the riboprobe distribution patterns compared. This would determine if the exon 4 riboprobe was localising only to MOR-1 or whether it was also localising to the MOR-1G - MOR-1L splice variants as well.

MOR-1 mRNA was readily detected within the Purkinje layer (panels A and E) of the adult rat cerebellum, the exon 4 riboprobe also localised to a sub-population of stellate and basket cells within the molecular layer of the cerebellum. The functional implications of locating MOR-1 mRNA in the cerebellum remains to be determined. The cerebellum is known to play a role in the learning, planning and execution of complex motor patterns, such as regulating postural balance and eye movement, as 
well as regulating body and limb movements. Recently the opioid receptors have been studied by opioid receptor gene inactivation in mice (Gaveriaux-Ruff and Kieffer 2002). MOR-knockout mice show strongly reduced or abolished locomotive abilities, implicating a role for MORs in the cerebellar functioning. The modification of locomotive ability in the MOR-knockout mice may also represent a unique function for the MORs in the development of the cerebellum.

\subsubsection{Exon 9 riboprobe}

The majority of FISH experiments performed using the exon 9 riboprobe resulted in what appeared to be non-specific staining (panel A), with no cytoplasmic staining visible. Weak staining of three structures within the adult rat brain was seen, the lateral reticular nucleus (Figure 3.16, panel A), the central nucleus of the inferior colliculus (Figure 3.16, panel $\mathrm{C}$ ) and the parvocellular lateral reticular nucleus (Appendix D). As with the exon 3 riboprobe, the exon 9 riboprobe would have been expected to localise to all the structures known to express the abundant MOR-1C and MOR-1D splice variants.

Only one batch of exon 9 riboprobes resulted in cytoplasmic staining (panel C) in a structure of the brain known to express the MOR-1C splice variant (Abbadie et al. 2000a). However the posthybridisation conditions were less stringent than the standard FISH conditions determined to give the highest stringency without loss of signal. In this case the third posthybridisation wash was performed for 15 minutes at $37^{\circ} \mathrm{C}$ in $2 \mathrm{x}$ SSC containing $40 \%$ deionised formamide, instead of for 20 minutes at $40^{\circ} \mathrm{C}$, the reduced stringency may account for the presence of signal (panel $\mathrm{C}$ ). 

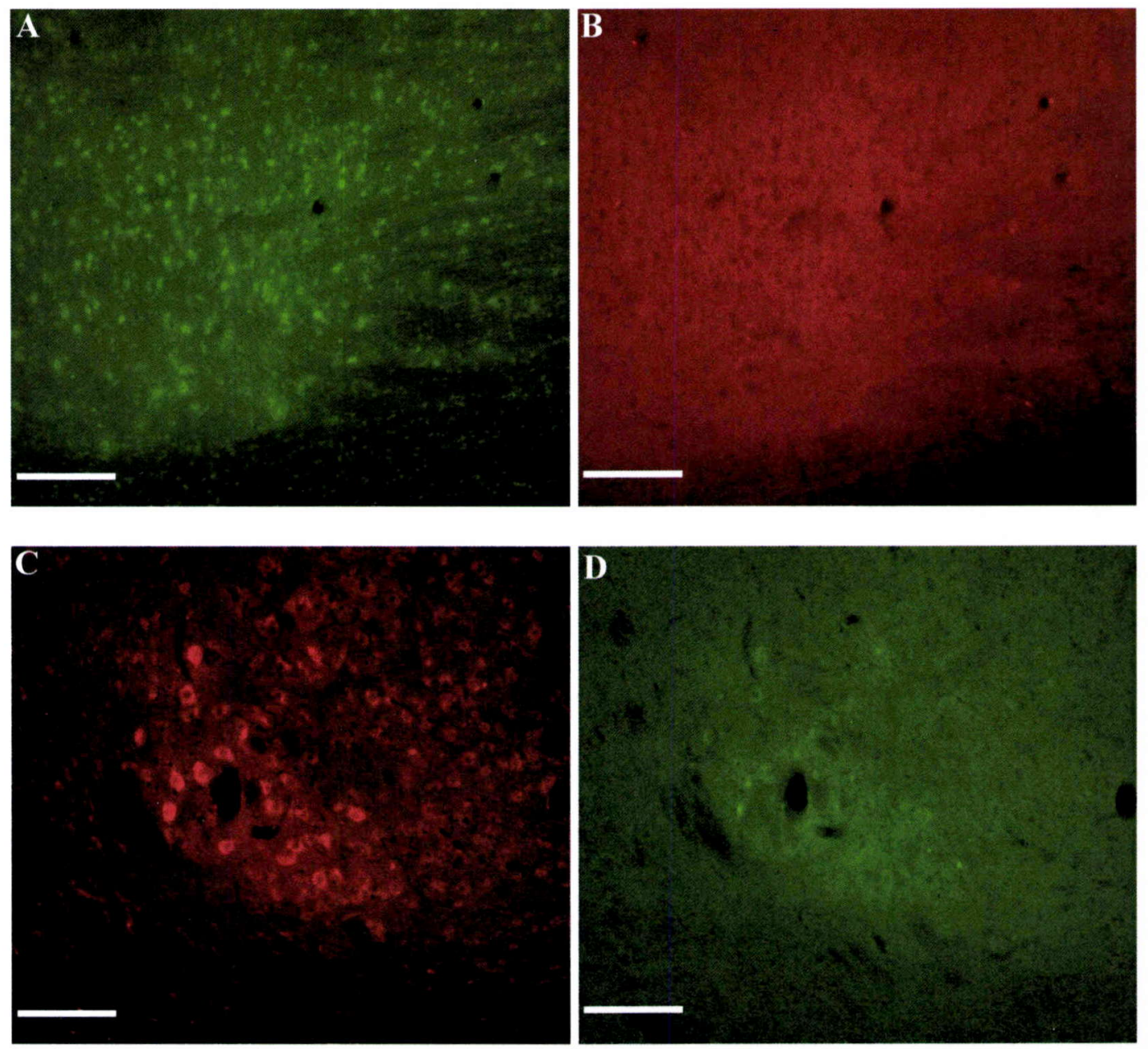

Figure 3.16 Exon 9 riboprobe localisation in adult rat brain. Fluorescent photomicrographs of sagittal sections through the lateral reticular nucleus ( $A$ and $B$ ) and the central nucleus of the inferior colliculus (C and D). Scale bar $=200 \mu \mathrm{m}$.

There also appears to be some tissue autofluorescence in the form of cytoplasmic staining (panel D), which could explain the intensity of the apparent exon 9 riboprobe localisation to the central nucleus of the inferior colliculus (panel C).

Reduced stringency typically results in an increase in non-specific interactions. Even though the signal in panel $\mathrm{C}$ appears to be localised to the cytoplasm, the fact that this riboprobe did not localise to the other structures in the brain known to express the MORs containing exon 9, suggests that the apparent localisation is most probably artifactual. 
The intention of this project was to elucidate the conditions under which all the riboprobes, including the exon 9 riboprobe, would specifically hybridise and then continue experimentations with suitable positive and negative controls to confirm specific localisation.

The exon 9 riboprobe was designed and synthesised toward the completion of this project and due to time constraints sufficient control experiments were not performed simultaneously with any of the exon 9 FISH experiments. Without rigorous control experiments it is difficult to have confidence that the staining visualised using this riboprobe, is specific for the targeted exon.

The exon 9 riboprobe was synthesised using 4 overlapping oligos, which may account for the lack of success experienced with this probe. The probability of mispriming and incorrect sequence incorporation is greatly increased by using more oligos to produce a riboprobe of 95 nts. An improved design for exon 9 riboprobe synthesis may produce more significant results than was experienced in this project.

One design proposal could be to clone the MOR-1C splice variant into a plasmid and create a forward and T7-tailed reverse primer specific to exon 9 . Therefore the ds template required for riboprobe synthesis would be generated by PCR, a method that has been successful in this project. Due to time constraints this option was not pursued. 


\subsubsection{Hae III generated Exon 1 riboprobe}

Exon 1 is present in 10 of the 15 MOR splice variants, 3 of which contain two potential translational start points in either exon 1 or exon 11 (MOR-1H - MOR1-J), (Pan et al. 2001).

This riboprobe was $117 \mathrm{nts}$ in length and as previously described it was generated by Hae III restriction digestion of the 287 bp MOR fragment (that ranges from the 5 , UTR of exon 1 to the end of exon 1). The exon 1 riboprobe showed staining to all structures within the brain known to contain MORs. Staining with this riboprobe occasionally appeared "cloudy" which is typical of non-specific interactions; this may have been due to the small size of the exon 1 riboprobe, poor riboprobe synthesis (refer Experimental Procedures) or poor tissue preparation. The Hae III exon 1 riboprobe appeared to be specific as increasing temperatures and more stringent hybridisation conditions abolished the probe hybridisation signal.

Figure 3.17 represents FISH experiments where the FITC-labelled exon 1 riboprobe has stained the cerebellum (panels A and C) and the dendate gyrus (panel D). In this project the granular layer of the cerebellum always appeared to show high background staining under all filter sets, this can be seen clearly in panel $\mathrm{C}$ which is a magnification of the image represented in panel A. The high background staining in the granular layer is probably due to the large cell density in this structure compared with other brain regions. 

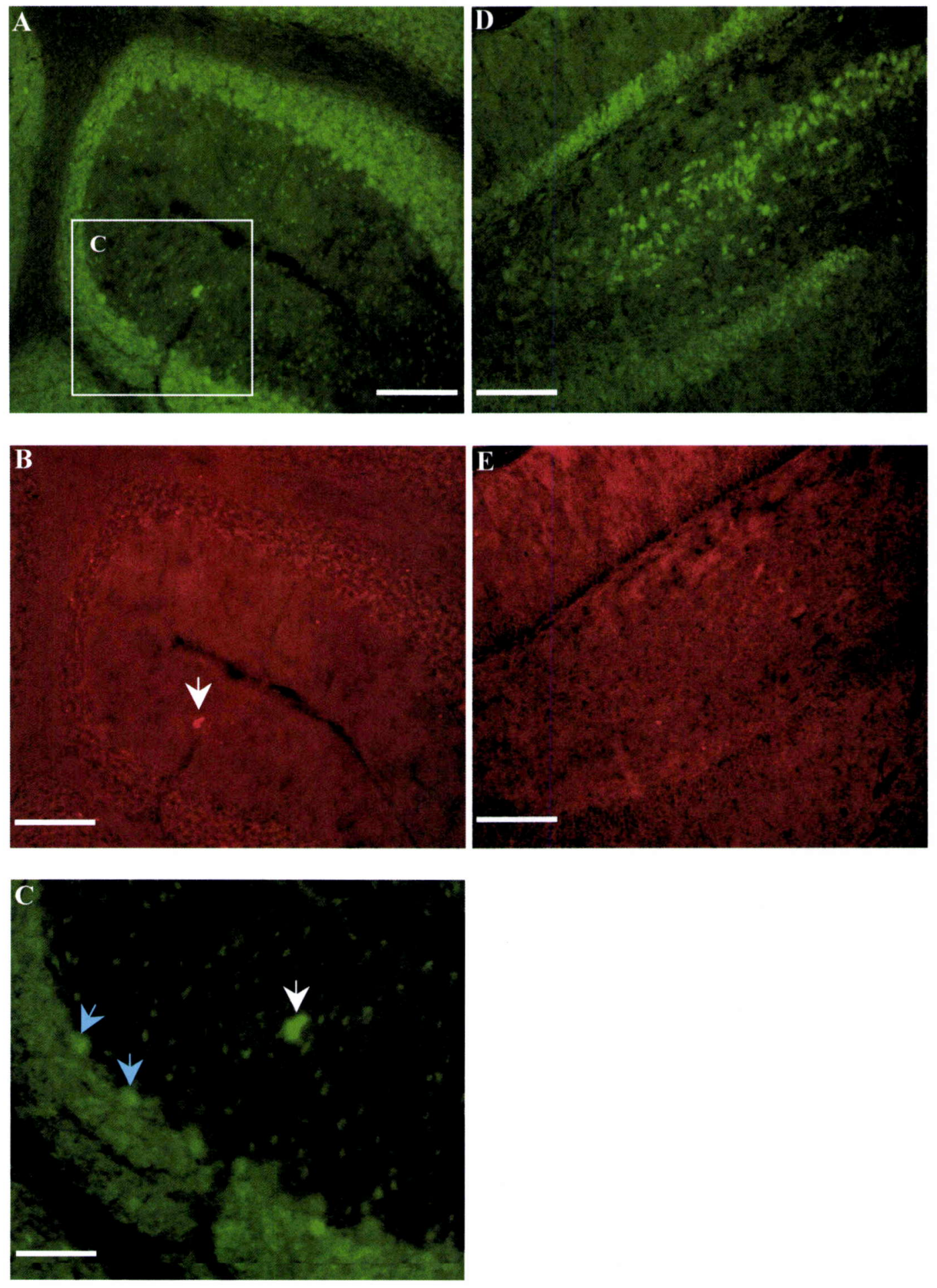

Figure 3.17 FITC-labelled exon 1 riboprobes. Fluorescent photomicrographs of sagittal sections through the cerebellum ( $\mathbf{A}, \mathbf{B}$ and $\mathbf{C}$ ) and the dendate gyrus ( $\mathbf{D}$ and E). The white arrows represent tissue autofluorescence (B and $\mathbf{C})$; the blue arrows show individual neurons within the Purkinje layer stained for exon 1 mRNA (C). Scale bar $=200 \mu \mathrm{m}$ (A, B, D and E), $50 \mu \mathrm{m}$ (C). 
A unique characteristic of the Hae III generated exon 1 riboprobe is that it was designed to a region of the Oprm (exon 1) that has very high sequence homology to the $\delta$ and $\kappa$ opioid receptors.

Further experiments would have to be designed to determine whether the exon 1 riboprobe was hybridising solely to MOR mRNA or to mRNA of the other opioid receptor subtypes. A competitive hybridisation experiment could be performed to determine if the Hae III generated exon 1 riboprobe was binding to the other receptor subtypes. An unlabelled exon 1 riboprobe could be designed and synthesised to DOR and utilised in a FISH with labelled Hae III exon 1 riboprobes specific to MOR. If the DOR displaced the staining visualised using the MOR exon 1 riboprobe, then one could determine that the Hae III generated exon 1 riboprobe was not specific for MOR. Conversely a labelled DOR exon 1 riboprobe and an unlabelled MOR Hae III exon 1 riboprobe could be used to determine the specificity of the riboprobe used in this project.

\subsubsection{Pvu II riboprobes}

The design of this 222 nt riboprobe complementary the 5' UTR + exon 1 ensured this riboprobe would hybridise preferentially to the MOR splice variants and not to the other receptor subtypes, as the 5' UTR is a unique region of the Oprm. Abolition of probe hybridisation signal using increasingly higher temperatures and more stringent hybridisation washes suggested that the $P v u I I$ generated riboprobe was interacting specifically. Photomicrographs were also captured under the opposite filter set to that which the probe was labelled with, these images demonstrated no signal, 
confirming that the signal being visualised is not tissue autofluorescence (Figure 3.18 panels $\mathrm{B}, \mathrm{D}$ and $\mathrm{F})$.

Staining with the $P v u I I$ riboprobes was consistent with other reports in the literature, they were shown to hybridise to the spinal trigeminal nucleus (Figure 3.18, panel A), areas 1 and 2 of the frontal cortex (Figure 3.18, panel C), anterior and posterior interposed cerebellar nucleus (Appendix D), dorsal endopiriform nucleus (Appendix D) and inferior olive (Figure 3.20a, panels D, E and F). In addition staining of the cerebellum was also seen (Figure 3.18, panel E) providing strong evidence for specificity of interaction for this riboprobe.

The opioid system has a well-defined role in nociception. In this project the localisation of riboprobes generated to exons 1,4 , including $P v u I I$ riboprobes and the 287 fragment to the spinal trigeminal nucleus (panel A) was in concordance with published data. The spinal trigeminal nucleus is the start point in the ascending trigeminal pathway and is involved in the mediation of facial pain and temperature. The exon 4, MOR 287 and the $P v u I l$ generated riboprobes were the only probes to localise to the facial nucleus in this project (results not shown); the significance of this is still to be determined.

Like the exon 4 riboprobes, the $P v u I I$ generated riboprobes also localised to individual neurons within the Purkinje and molecular layers of the cerebellum, as well as to a sub-population of stellate and basket cells within the molecular layer (panel E). 

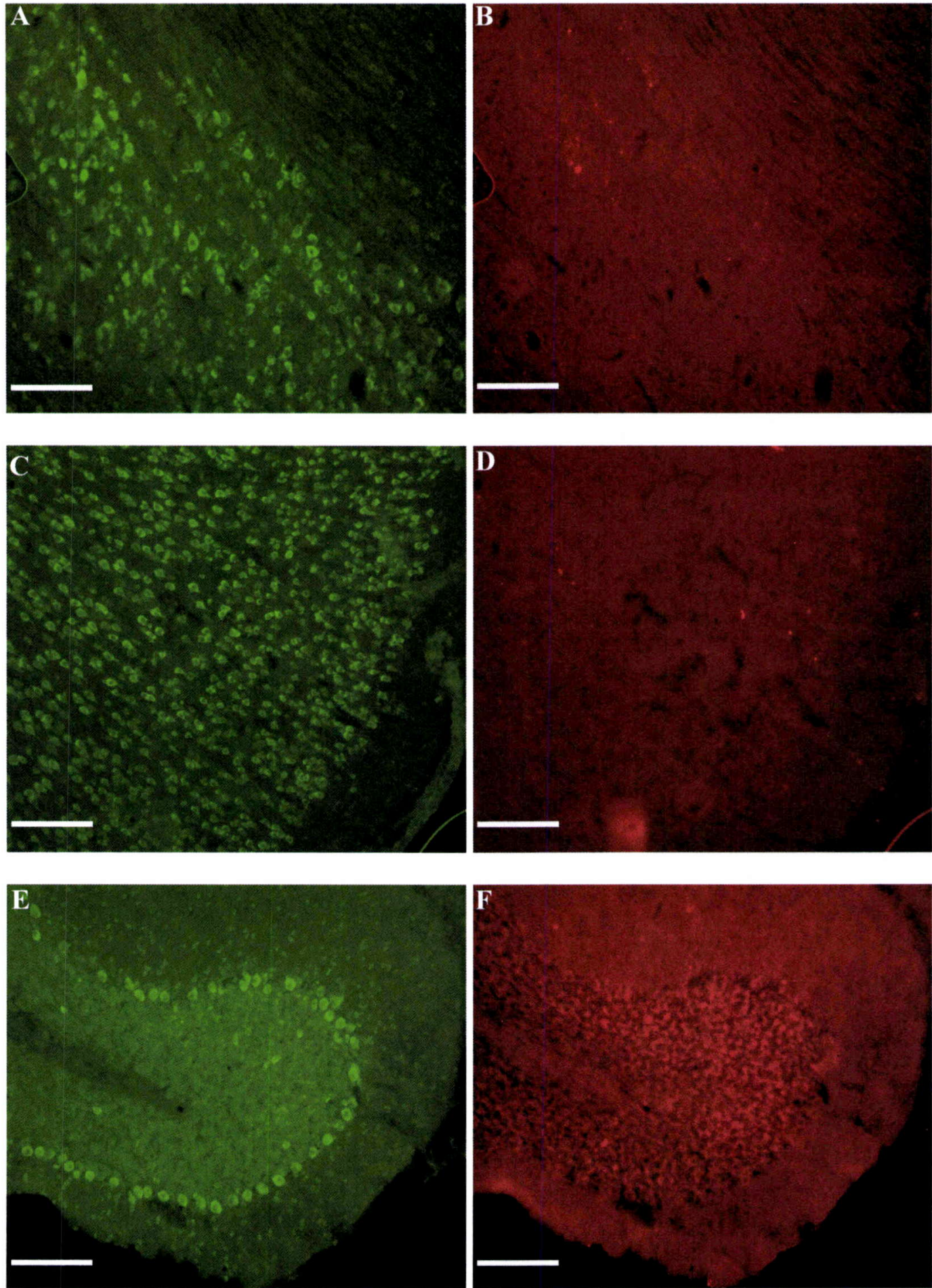

Figure 3.18 FITC-labelled $P v u$ II generated riboprobes. Fluorescent photomicrographs of sagittal sections through the spinal trigeminal nucleus ( $A$ and B), frontal cortex area 1 (C and D) and the cerebellum (E and F). Scale bar $=200$ $\mu \mathrm{m}$. 


\subsubsection{Differential Distribution}

A secondary intention of this project was to try and map any differential distribution of the MOR splice variants using the exons 1, 3,4 and 9 riboprobes. However, as only the exons 1, 4, Pvu II and MOR 287 fragment riboprobes produced what appeared to be specific staining, only these riboprobes were used to study the distribution of MOR mRNA in this project.

Differential staining of exon $4 \mathrm{mRNA}$ and the $P v u I I$ generated fragment and the MOR 287 fragment mRNA was observed in the cerebellum (Figure 3.19a and Figure 3.19b), frontal cortex area 2, inferior olive (Figure 3.20a and Figure 3.20b), interposed cerebellar nucleus, spinal trigeminal nucleus (Figure 3.21), rhinal fissure, and the dendate gyrus.

\section{Cerebellum}

Figure $3.19 \mathrm{a}$ and $3.19 \mathrm{~b}$ show staining with various riboprobes to the Purkinje and molecular layers of the cerebellum. Unique to these images is the appearance of two layers of Purkinje cells within the Purkinje layer of the cerebellum (Figure 3.19a panel F, represented by white arrows), historically only one layer has ever been reported. It is possible that this other layer does exist, and interestingly it was only observed when utilising the exon 4 riboprobe, which was probably hybridising to MOR-1 mRNA (over the less abundant MOR-1G - MOR-1L splice variants). 

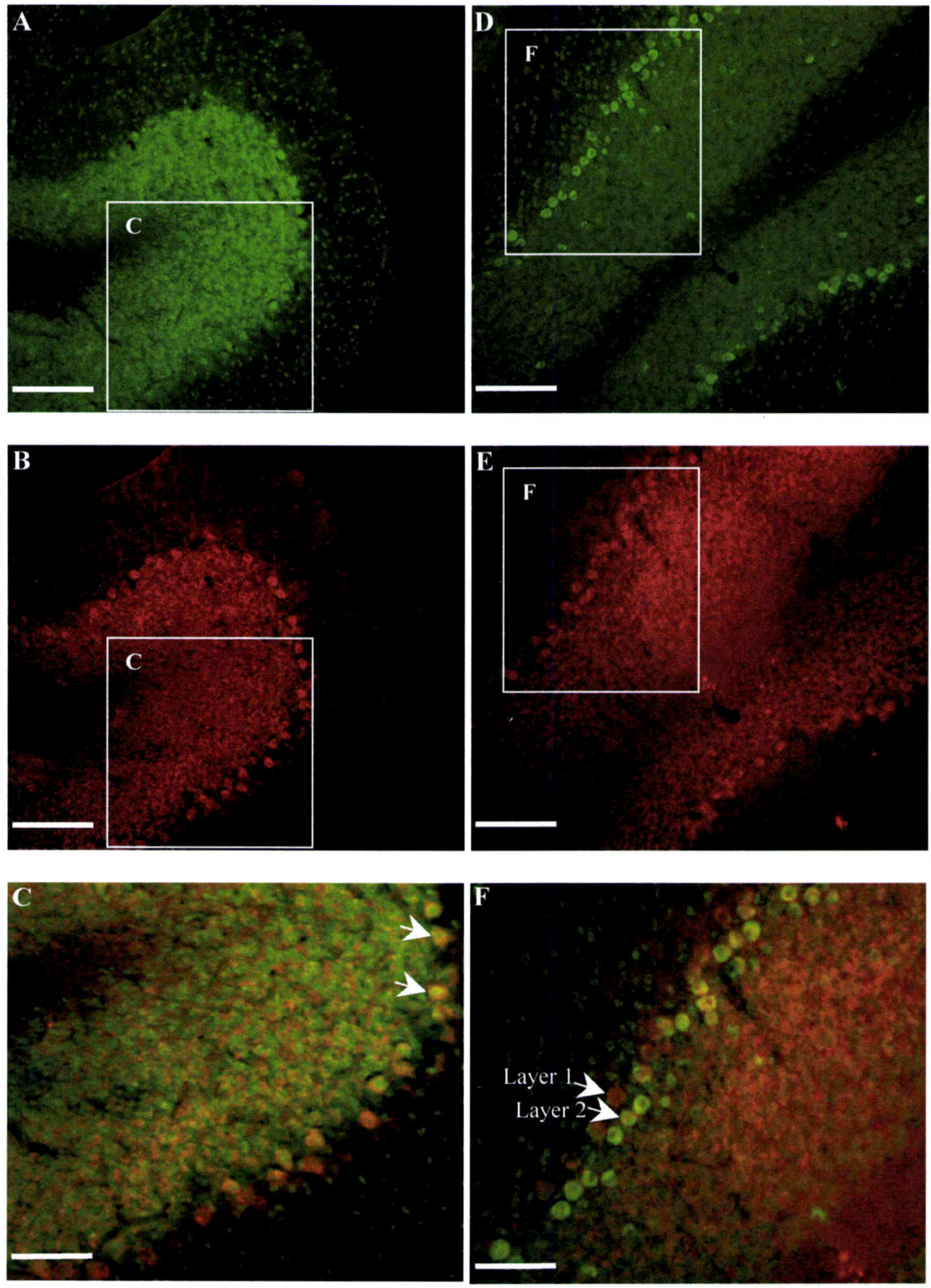

Figure 3.19a Differential staining of CY3-labelled exon 4 riboprobes and FITClabelled riboprobes. Fluorescent photomicrographs of sagittal sections through the cerebellum. The exon 1 (A) riboprobes and the exon 4 (B) riboprobes appear yellow where they colocalise (C, depicted by white arrows). Whereas the Pvu II generated riboprobes (D) and the exon 4 riboprobes $(E)$ appear to show differential staining $(\mathbf{F})$. The white arrows depicted in panel $\mathbf{F}$ represent the outer and inner layers of Purkinje cells recognised by the exon 4 and Pvu // generated riboprobes, respectively. Scale bar $=200 \mu \mathrm{m}(\mathbf{A}, \mathbf{B}, \mathbf{D}$ and E), $150 \mu \mathrm{m}(\mathbf{C}), 100 \mu \mathrm{m}(\mathbf{F})$. 

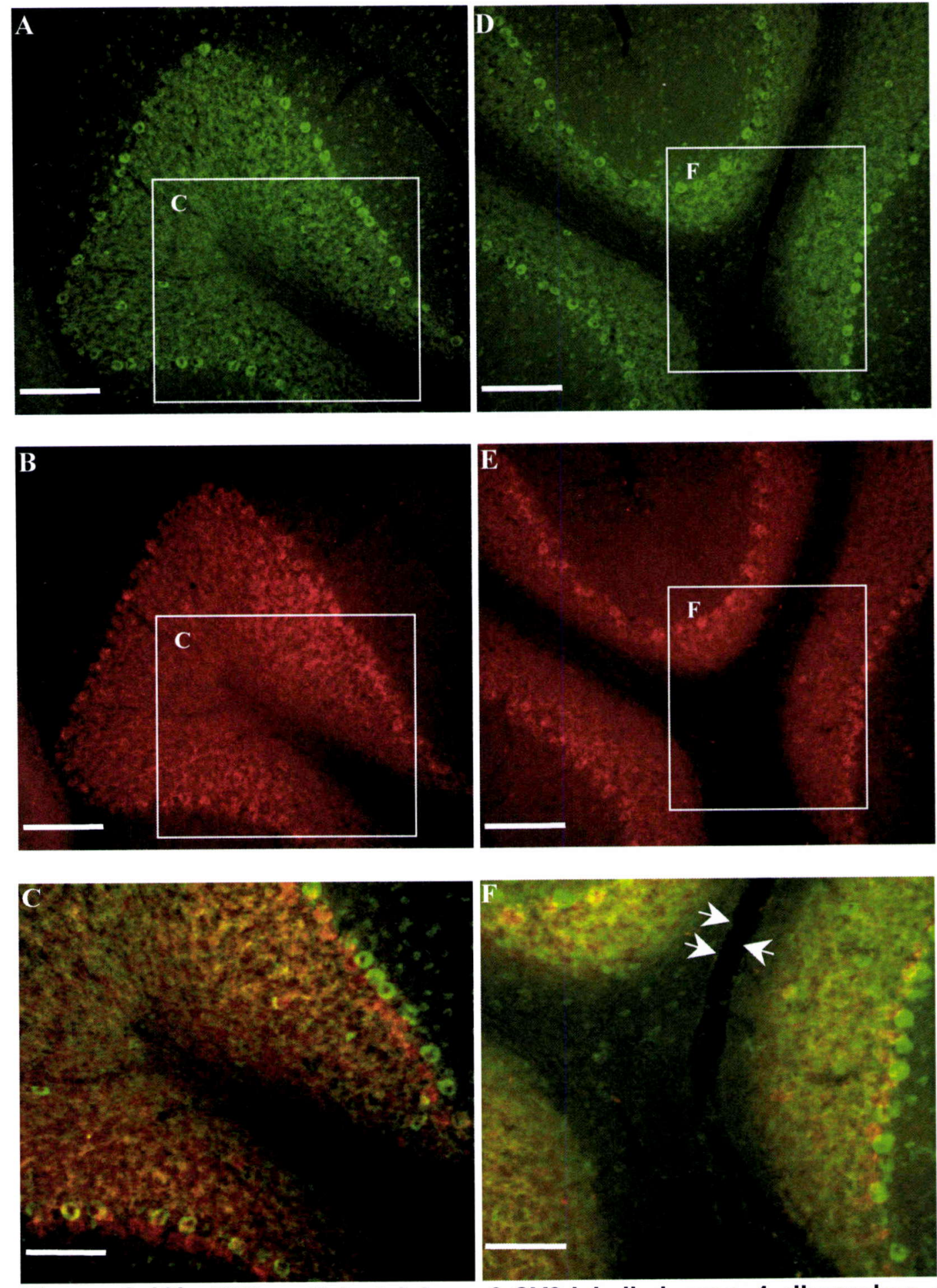

Figure 3.19b Differential staining of CY3-labelled exon 4 riboprobes and FITC-labelled 287 fragment riboprobes. Sagittal sections through the cerebellum. The FITC-labelled 287 fragment riboprobes have localised to Purkinje cells and stellate or basket cells within the molecular layer (A and D). The CY3-labelled exon 4 riboprobes have localised mainly to the Purkinje cells and can be seen to hybridise to some cells within the granular layer ( $\mathbf{B}$ and $\mathbf{E}$ ). Panels $\mathbf{C}$ and $\mathbf{F}$ are the enlarged overlaid images, where one can clearly see differential distribution of the two riboprobes. The white arrows show perfect tissue/image alignment $(\mathbf{F})$. Scale bar $=200 \mu \mathrm{m}(\mathbf{A}, \mathbf{B}, \mathbf{D}$ and E), $100 \mu \mathrm{m}(\mathbf{C}$ and F). 
However as no other study reports the presence of two layers of Purkinje cells, this double layer could perhaps be explained as an artefact of the tissue preparation. The tissue was cut $12 \mu \mathrm{m}$ thick, although it is possible that the tissue sections were thicker than was intended, due to cutting technique or the orientation of the tissue on the cryostat chuck. This could give the appearance of a double layer, if signal from riboprobes hybridised deep to superficial layer Purkinje cells penetrated through the tissue, giving the appearance of an outermost layer.

Initial examination of the overlaid images revealed what appeared (Figure 3.19a and 3.19b panels $\mathrm{C}$ and $\mathrm{F}$ ) to be tissue or image misalignment. Upon closer inspection one is able to see perfect alignment around a tear in the tissue (Figure $3.19 \mathrm{~b}$ panel F, represented by white arrows). So the differences seen in riboprobe distribution are unlikely to be due to tissue or image misalignment.

The signal detected is not likely to be fluorescence quenching as this phenomenon relies on high loading concentrations or high labelling densities of the probes being used (Gregory 1996). Approximately $6 \mathrm{ng}$ of riboprobe was added to each hybridisation reaction, which is not a high loading concentration and the labelling densities of each riboprobe, regardless of the labelling ratio used, was very low due to the short length of all the riboprobes in this project.

Another factor that influences fluorescence quenching is the proximity of the excited fluorophore to the acceptor molecule. It is generally accepted that in order for the energy transfer to occur the acceptor molecule must reside physically close (generally in large clusters) to the excited fluorophore (Abramowitz et al. 2003). 
Figure 3.19a, panel F represents distinct distribution of the two riboprobes, where one can clearly see that the Purkinje cells are neither in clusters nor molecularly physically close to one another.

Areas where both probes have colocalised to the same neuron appear yellow once the images are overlaid (Figure 3.19a, panel C). Interestingly the only experiments where the exon 4 riboprobe appears to always colocalise to the same neuron are those performed with the Hae III generated exon 1 riboprobe.

\section{Inferior Olive}

The inferior olive (also known as inferior olivary nucleus) is located lateral to the pyramidal tract on the medulla and receives sensory and motor information, via numerous tracts about the movements the cerebellum has just performed. This information is processed by the inferior olive and sent back to the cerebellum via the inferior cerebellar peduncle to inform the cerebellum how well the movement was performed (Hendelman 1994).

All four riboprobes used in the differential distribution experiments localised to the inferior olive (Figures $3.20 \mathrm{a}$ and $3.20 \mathrm{~b}$ ). Localisation of the exon 4 riboprobe to this region may suggest that the MOR-1G - MOR-1L splice variants are also expressed here. However, further experimentation would be required to support this supposition. 

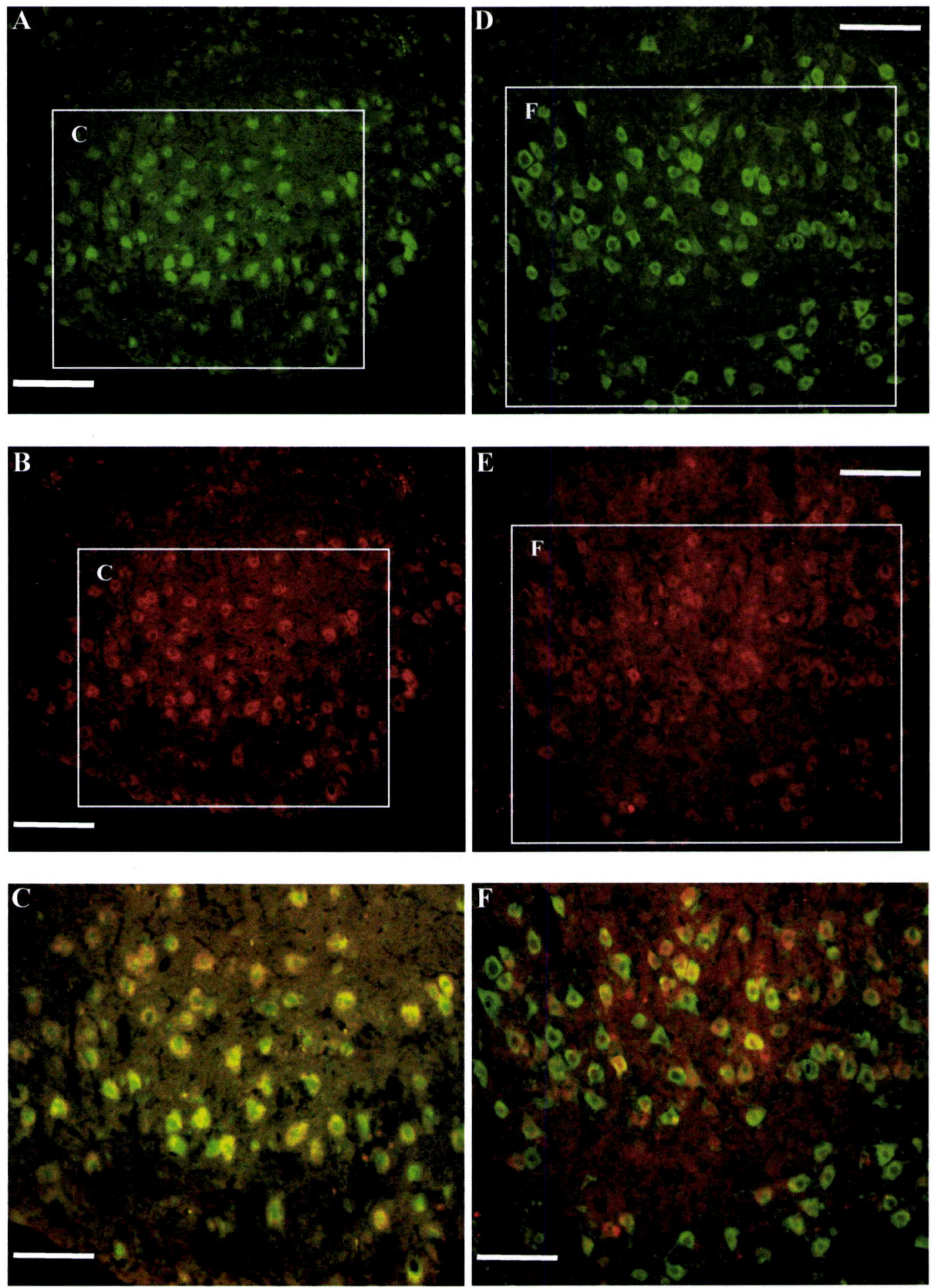

Figure 3.20a FISH images of CY3-labelled exon 4 riboprobes and FITClabelled exon 1 (Panels A, B, C) and Pvu II generated riboprobes (Panels D, E, F). Fluorescent photomicrographs of sagittal sections through the inferior olive. The Hae III generated FITC-labelled exon 1 riboprobe (A) appears to colocalise to all neurons expressing exon 4 (B) within the inferior olive (C). Whereas the Pvu II generated riboprobes (D) appears to be differentially expressed to the CY3-labelled exon 4 riboprobes $(E)$ in the neurons of the inferior olive $(\mathbf{F})$. Scale bar $=200 \mu \mathrm{m}$ (A, B, D and E), $150 \mu \mathrm{M}(\mathbf{C}), 175 \mu \mathrm{m}(\mathbf{F})$. 

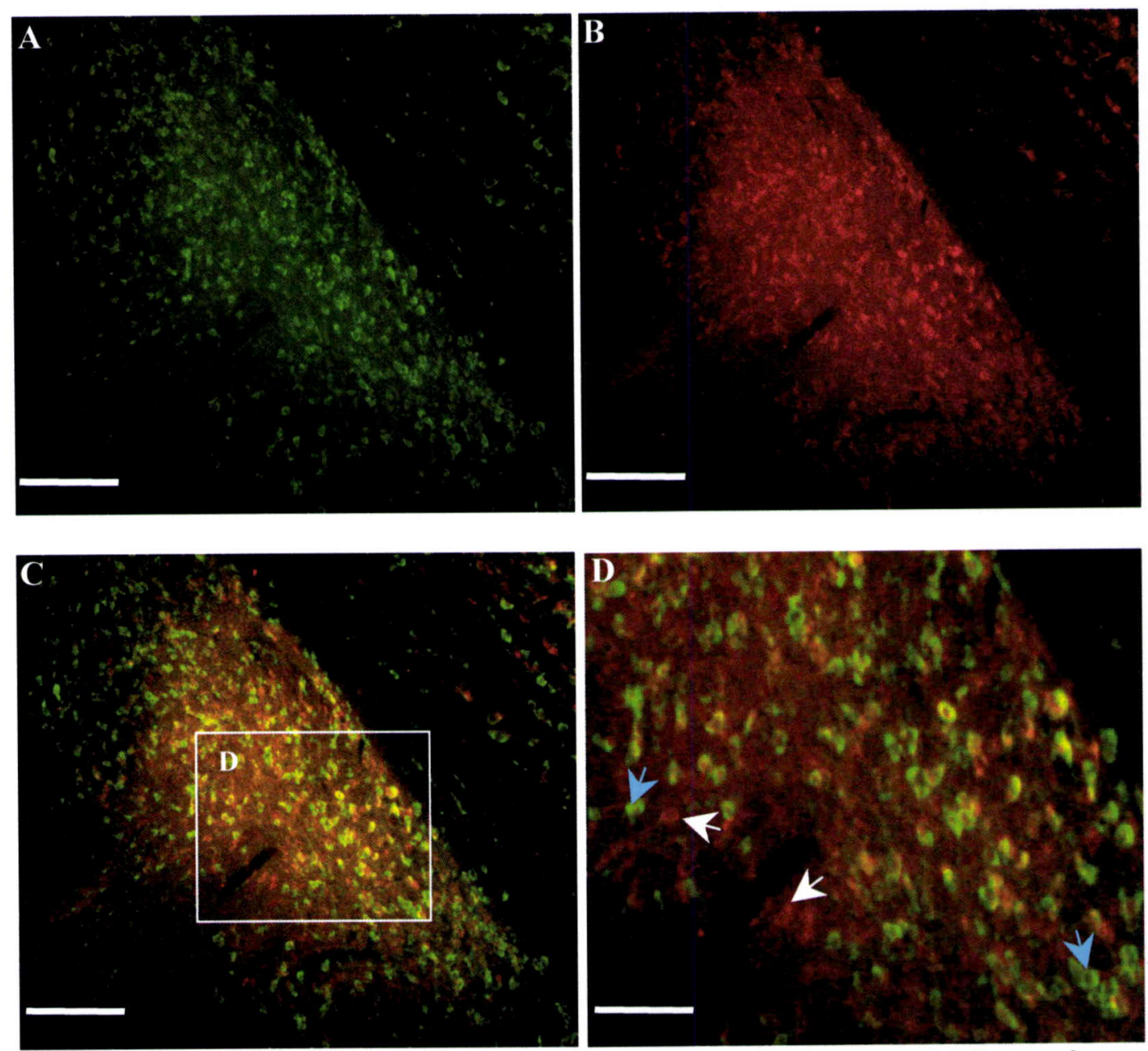

Figure 3.20b Differential staining of CY3-labelled exon 4 riboprobes and FITC-labelled MOR 287 fragment riboprobes in the inferior olive. These images were captured under the $10 x$ objective. Both the MOR 287 fragment riboprobes (A) and exon 4 riboprobes $(\mathbf{B})$ are dispersed throughout the inferior olive (C). At higher magnification (D) it is possible to see differential distribution of these two riboprobes. The arrows in panel $\mathbf{D}$ represent neurons stained only for exon 4 and blue arrows represent neurons stained only for the MOR 287 fragment mRNA. Scale bar $=400$ $\mu \mathrm{m}(\mathbf{A}, \mathbf{B}$ and $\mathbf{C}), 200 \mu \mathrm{m}$ (D).

Figures 3.20a, panel F and Figure 3.20b panels C and D show differential staining of the riboprobes, where there is clear cytoplasmic staining in both cases. The exon 4 riboprobe appears to be expressed in certain neurons of the inferior olive where the MOR 287 fragment riboprobes are not (white arrows, Figure 3.20b, panel D) and vice versa (blue arrows, Figure $3.20 \mathrm{~b}$, panel D). One very important consideration that is highlighted by Figures 3.20a, panels B and E, is signal intensity. The short length of the riboprobes utilised in this project produced many technical difficulties, not only in purification but also in discrimination between artefacts and valid signal. 
To generate a CY3-labelled exon 4 riboprobe, a labelling ratio of 1:5, CY3UTP:UTP was used; this resulted in $5 \%$ of the exon 4 riboprobe being labelled with CY3-UTP. Because these short riboprobes are not densely labelled the signal visualised can be very close to the limits for signal detection, due to the signal being so close to the baseline or background noise levels.

This phenomenon is also illustrated by the MOR 287 fragment riboprobe; the reduction in signal intensity is evident as this fragment is digested to form smaller riboprobes. The $222 \mathrm{nt} P v u I I$ riboprobe produces signals at almost an equal intensity to the MOR 287 fragment riboprobe, whereas the Hae III generated exon 1 riboprobe (which is $117 \mathrm{nt}$ in length) produces a marked reduction in signal intensity (Figure 3.21, panels $\mathrm{C}$ and $\mathrm{F}$ ). The reduction in signal intensity is most probably due to the decrease in $T_{m}$ and specific content, as well as the decrease in specific activity due to the short length of these riboprobes.

\section{Spinal Trigeminal Nucleus}

In this project the exon 4 riboprobe and the MOR 287 fragment riboprobe localised to different neurons in the spinal trigeminal nucleus (Figure 3.21, panel F). But again the exon 1 riboprobe colocalised with the exon 4 riboprobe (Figure 3.21, panel C).

The only difference between the $P v u I I$ generated riboprobe and the Hae III generated exon 1 riboprobe is the absence of the 5' UTR. The 5' UTR was cleaved off the MOR 287 fragment by restriction digestion using Hae III, and without the 5' UTR the exon 1 riboprobe exhibits colocalisation. There are several explanations for 
this phenomenon, it is possible that as mentioned in section 3.4 .8 , the exon 1 riboprobe is localising to the $\delta$ and $\kappa$ opioid receptors, competitive hybridisation experiments using riboprobes to exon 1 of DOR and KOR would determine if the MOR exon 1 riboprobe was binding to these other opioid receptor subtypes.

An essential part of spatial control of gene expression is mRNA localisation; a general model for mRNA localisation by active transport involves mRNA recognition, followed by association of a core ribonucleoprotein complex containing motor proteins for deliverance of the mRNA to the destination site. mRNAs can be localized by local protection from degradation, diffusion to a localised anchor, and by active transport (Palacios and St Johnston 2001). mRNA localisation typically requires specific cis-acting signals that are generally found in the 3' UTR, however recent examples indicate that these cis-acting signals can also be located in the 5' UTR (Jansen 2001).

It is therefore possible that the differential staining visualised using riboprobes to exon 4 and the MOR 287 fragment is due to the hybridisation of the MOR 287 and $P v u$ II riboprobes to mRNA destined for localisation in the Purkinje cell layer of the cerebellum. To test this hypothesis one could differentially label one riboprobe to the 5' UTR and the other to exon 1. The results of this FISH should prove whether the riboprobes in this project were hybridising to mRNA destined for the Purkinje cell layer and the translated mRNA of the Purkinje cells. As differential expression of a 5' UTR riboprobe and exon 1 riboprobe would indicate the difference between untranslated mRNA (possibly prior to localisation) and translated mRNA minus the 5' UTR. 

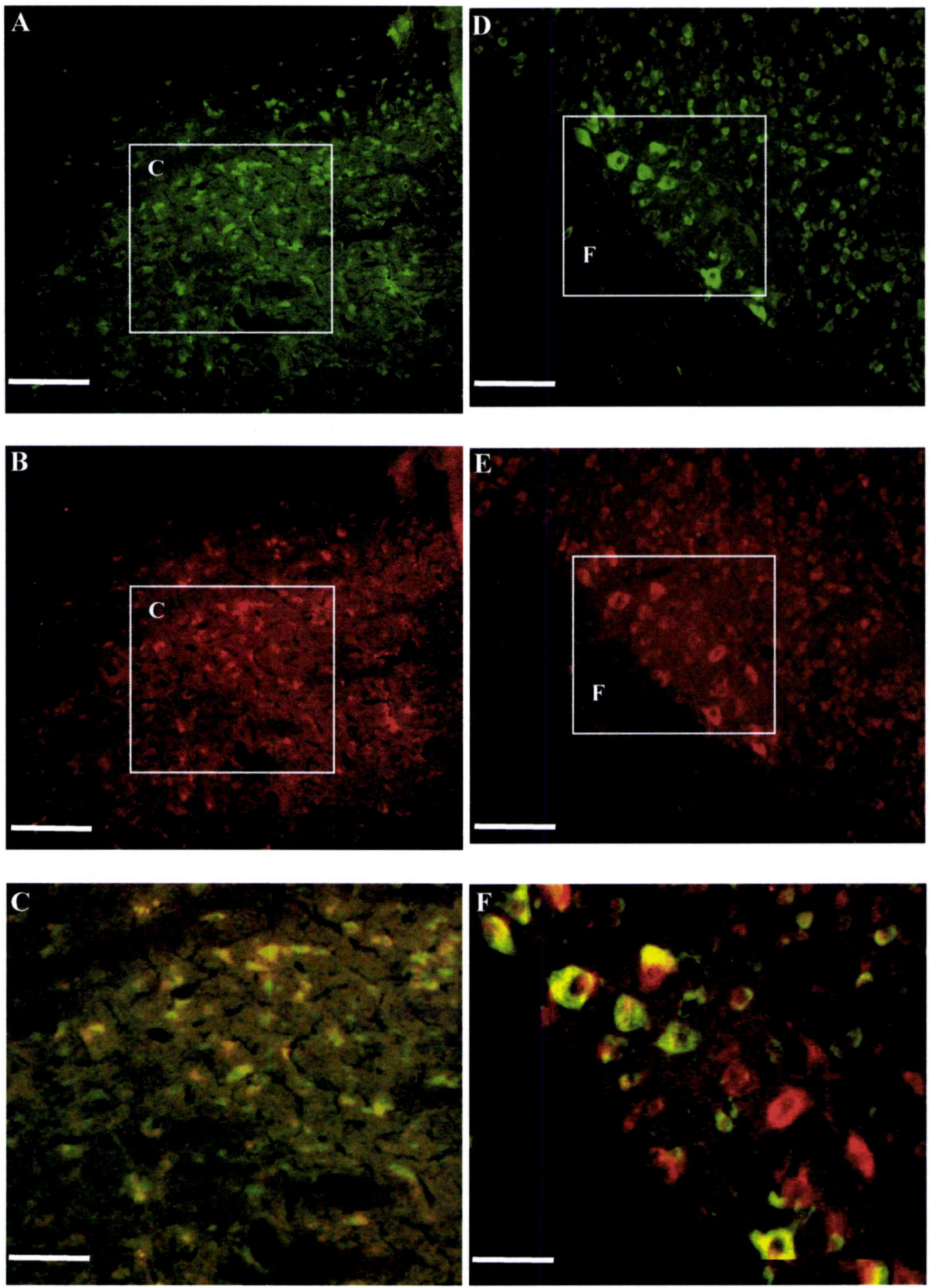

Figure 3.21 Differential staining of CY3-labelled exon 4 riboprobe and FITClabelled exon 1 and MOR 287 fragment riboprobes in the spinal trigeminal nucleus. The exon 4 (A) and exon 1 (B) riboprobe colocalised to neurons and axonal processes in the spinal trigeminal (C). Whereas the exon 4 (D) and MOR 287 fragment (E) riboprobe appears to be differentially distributed in the neurons of the spinal trigeminal nucleus $(\mathbf{F})$. Scale bar $=200 \mu \mathrm{m}(\mathbf{A}, \mathbf{B}, \mathbf{D}$ and $\mathbf{E}), 100 \mu \mathrm{m}(\mathbf{C}$ and $\mathbf{F}$ ). 


\subsection{Conclusions}

Without further experimentation it is impossible to support the hypothesis that the exon 4, $P v u I I$ generated and MOR 287 fragment riboprobes are localising to different MOR splice variants, such as MOR-1G and MOR-1, respectively.

As mentioned MOR-1G - MOR-1L splice variant distribution has only been examined using RT-PCR, a sensitive technique that involves the amplification of the target. These splice variants have been reported to be present in very low amounts and as such, it is highly unlikely that the riboprobes utilised in this project were detecting these less abundant splice variants.

The results of this project also suggest that FISH could be used to track the distribution patterns of untranslated and translated mRNA, however further experimentation would be required to determine if these results could be repeated using fresh frozen tissue.

The identification of MOR mRNA in the cerebellum is in concordance with previous experiments conducted by Eli Mrkusich in the same laboratory (Mrkusich et al. 2003). The exon 1, Pvu II, MOR 287 fragment and exon 4 riboprobes designed and synthesised in this project, all localised to Purkinje cells in the Purkinje layer, basket cells and stellate cells in the molecular layer of the cerebellum.

The presence of two layers of Purkinje cells in the Purkinje layer of the cerebellum has not been reported before and may be due to the presence of untranslated mRNA destined for the Purkinje cell layer. This data was reproduced in three separate 
experiments using two different rat brains, but could only be considered significant if these results could be reproduced using fresh frozen tissue, as the background and amount of artefacts encountered are greatly reduced using this tissue.

Overall I believe the use of these new novel short riboprobes is a feasible, but technically difficult approach for studying the distribution patterns of the MOR splice variants. The problems encountered using short riboprobes were numerous; including low specific content, difficulty in purification, reduced labelling density and difficulty designing positive controls. However short riboprobes are still more effective than short oligos, as the hybrids produced using oligos are less stable than riboprobe hybrids, oligos have limited labelling methods and have a much lower specific activity than riboprobes (Feldman et al. 1997).

\subsection{Future work}

Future work on the analysis of MOR splice variant distribution should include the design and synthesis of a sense riboprobe to each of the riboprobes used in this project. The utilisation of a sense riboprobe in FISH experiments should result in the absence of signal, this result would confirm the specificity of each riboprobe for its target.

Further research utilising the riboprobes designed in the present study is required, before any conclusions can be made about the possible significance of the differential distribution discovered. As the specific roles of each splice variant remains unknown, one could only hypothesise that the differential distribution may, as in other cases (Forlano et al, 2000), have a functional role in development. All 
things considered, the identification of differential distribution by FISH using small fluorescently labelled riboprobes designed to exons of the Oprm, provides support to the current hypotheses that the splice variants of the opioid system are differentially distributed and have unique, if not overlapping, functions (Abbadie et al. 2001).

My future recommendation for this project would be to produce riboprobes specific to exons of the Oprm that are approximately $150 \mathrm{nt}$ in length, by PCR off a plasmid containing the target sequence of interest. Approximately $25 \%$ of a $150 \mathrm{nt}$ riboprobe would be labelled using a 1:5 labelling ratio. This would result in a higher signal to background ratio aiding in the interpretation of localisation data.

This project highlights the limitations of using FISH as a quantitative method, because short riboprobes produce signal very close to background levels. More information could be obtained about mRNA transcript levels if FISH were combined with a signal amplification method (such as antibodies or TSA). Even though this would result in the loss of quantitative data produced by FISH, much better localisation signals could be obtained aiding the in situ analysis of MOR splice variant distribution in rat brain. 


\section{Chapter 4}

\section{Experimental Procedures}

\subsection{Primer synthesis}

All primers and overlapping oligonucleotides were synthesised by Invitrogen ${ }^{\mathrm{TM}}$ life technologies, the scale of synthesis was 50 nmole and purification was achieved by desalting. Table 4.1 gives the sequences of primers used to generate the MOR-1 specific CLiP and MOR-1 target template.

\begin{tabular}{|l|l|}
\hline Name of Product & Product Sequence \\
\hline MOR.A1.Rev & 5' BIOTIN CCA GTT GCA GAC GTT GAC GAT TT 3' \\
\hline MOR.A2.Rev & $\begin{array}{l}\text { 5' CCA GTT GCA GAC GTT GAC GAT TTC GTT } \\
\text { GTC AGA AGT AAG TTG 3' }\end{array}$ \\
\hline MOR.B1.Fwd & 5' ATC CTC TCT TCT GCC ATC GGT CT 3' \\
\hline MOR.B2.Fwd & $\begin{array}{l}\text { 5' ATC CTC TCT TCT GCC ATC GGT CTA CGG } \\
\text { ATG GCA TGA CAG T 3' }\end{array}$ \\
\hline MOR.B3.Fwd & $\begin{array}{l}\text { 5' ATC CTC TCT TCT GCC ATC GGT CTA CGA } \\
\text { GTG GGT TAC ATC GA 3' }\end{array}$ \\
\hline MOR.794.Fwd & 5' CCT GCC GCT CTT CTC TGG TTC 3' \\
\hline MOR.794.Rev & 5' CCT GCC TGT ATT TTG TGG TTG 3' \\
\hline
\end{tabular}

Table 4.0 Primer sequences used to generate CLiPs and MOR-1 target template. CLiP primer sequences are shown in black type. Nucleotides complimentary to PBSSK are shown in bold type and the MOR-1 target specific 'tail' is represented by normal black type. The primers used to generate the MOR-1 target for CLiP ligation in solution, are named MOR.794 forward or reverse. The '794' denotes the size of the product in base pairs. NB The phosphorylated primer is MOR.B1.Fwd. 
Table 4.1 shows the primer sequences used to generated riboprobes to exons 3,4 and 9 of the Oprm gene. The MOR 287 fragment was generated by PCR using the MOR.794.Fwd primer (Table 4.0) and the MOR.287.T7.Rev primer (Table 4.1).

\begin{tabular}{|l|l|}
\hline Name of Product & Product Sequence \\
\hline *MOR.Ex3.primer.Fwd & 5' CCT TCA TCA TGC CGG TCC T 3' \\
\hline *MOR.Ex3.T7.primer.Rev & $\begin{array}{l}\text { 5' TAA TAC GAC TCA CTA TAG GGT GCG } \\
\text { GAC ACT CTT GAG TCG T 3' }\end{array}$ \\
\hline \#MOR.Ex4.Fwd.2 & $\begin{array}{l}\text { 5' GAG GCT GCC ATC TAC TTG GAA TCA GGT } \\
\text { TGC TGT CAG GGT TTG TGG 3' }\end{array}$ \\
\hline \#MOR.Ex4.T7.Rev.2 & $\begin{array}{l}\text { 5' TAA TAC GAC TCA CTA TAG GGA GCG } \\
\text { CCT CCC ACA AAC CCT GAC AGC A 3' }\end{array}$ \\
\hline \#MOR.Ex9.A.Fwd & $\begin{array}{l}\text { 5' TTC ACT CAT AAA GGT TAG GAG TTG TAA } \\
\text { GTG TAA TGT C 3' }\end{array}$ \\
\hline \#MOR.Ex9.B.Fwd & $\begin{array}{l}\text { 5' ATG TAT TGT CTG GTT TGC CGT AAT GCG } \\
\text { TGT TTT CAT CC 3' }\end{array}$ \\
\hline \#MOR.Ex9.C.Rev & $\begin{array}{l}\text { 5' GCA AAC CAG ACA ATA CAT TAG ACA TTA } \\
\text { CAC TTA CAA CT 3' }\end{array}$ \\
\hline \#MOR.Ex9.D.Rev & $\begin{array}{l}\text { 5' TAA TAC GAC TCA CTA TAG GGA TGA } \\
\text { AAA CAC GCA ATA 3' }\end{array}$ \\
\hline MOR.287.T7.Rev & $\begin{array}{l}\text { 5' TAA TAC GAC TCA CTA TAG GGC GCA } \\
\text { TGG ATC GGA CTG GTG 3' }\end{array}$ \\
\hline
\end{tabular}

Table 4.1 Sequences used to generate riboprobes to specific exons of MOR (e.g. Ex3, exon 3). Nucleotides complimentary to the T7 RNA promoter sequence are shown in bold. The incorporation of this sequence allows for the synthesis of single-stranded riboprobes using the DNA-dependent T7 RNA polymerase (Invitrogen ${ }^{\mathrm{TM}}$ life technologies). * Denotes primers and \# denotes overlapping oligonucleotides. MOR.287.T7.Rev is an internal reverse primer located within exon 1 of the Oprm gene.

\subsection{Phosphorylation of primers}

Primers were phosphorylated as previously described (Day et al. 1995). Briefly, 1 nmole of primer was incubated for 45 minutes at $37^{\circ} \mathrm{C}$ with 10 Units of $\mathrm{T} 4$ polynucleotide kinase (Invitrogen ${ }^{\mathrm{TM}}$ life technologies), in $70 \mathrm{mM}$ Tris- $\mathrm{HCl} \mathrm{pH}$ 7.6 Forward Reaction Buffer, containing $10 \mathrm{mM} \mathrm{MgCl}$, $100 \mathrm{mM} \mathrm{KCl,} 1 \mathrm{mM}$ 2-mercaptoethanol 
and $500 \mu \mathrm{M}$ ATP in a final volume of $50 \mu \mathrm{L}$. The T4 polynucleotide kinase was heat inactivated at $95^{\circ} \mathrm{C}$ for 10 minutes; these primers could then be used in a PCR for CLiP synthesis.

\subsection{CLiP synthesis}

\subsubsection{Restriction digestion of pBSSK$^{-}$containing the 5' UTR and exon 1 of Oprm}

pBluescript $^{\circledR} \mathrm{SK}^{-}$(pBSSK$)^{-}$) phagemid (Stratagene) is a 2958 bp plasmid that confers ampicillin resistance and has a multiple cloning site flanked by T3 and T7 RNA polymerase promoters. E.coli cells transformed with $\mathrm{pBSSK}^{-}$plasmids containing the 5' UTR and exon 1 of the Oprm gene were kindly gifted by Eli Mrkusich. This plasmid was linearised by restriction digestion to be used as template for the first round PCR to produce the ds CLiP template (4.2.2). $1 \mu \mathrm{g}$ of $\mathrm{pBSSK}^{-}$was digested

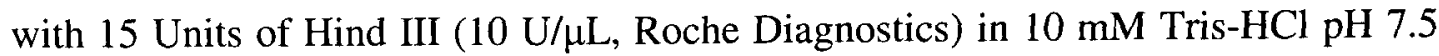
containing $10 \mathrm{mM} \mathrm{MgCl}, 50 \mathrm{mM} \mathrm{NaCl}, 1 \mathrm{mM}$ 1,4-Dithioerythritol (DTE) for 4 hours at $37^{\circ} \mathrm{C}$. After the digestion heating the sample to $65^{\circ} \mathrm{C}$ for 15 minutes inactivated the restriction endonuclease.

\subsubsection{PCR synthesis of CLiP template}

Various size CLiPs were synthesised by PCR amplification using differing primer pairs (Table 4.1 ) to the linearised pBSSK: Each $50 \mu \mathrm{L}$ CLiP template PCR contained $400 \mathrm{nM}$ each forward and reverse primers and $5 \mathrm{ng}$ of the linearised plasmid. The reaction buffer was $20 \mathrm{mM}$ Tris- $\mathrm{HCl}(\mathrm{pH} \mathrm{8.4)} \mathrm{containing} 50 \mathrm{mM} \mathrm{KCl}$, $1.5 \mathrm{mM} \mathrm{MgCl}_{2}, 200 \mu \mathrm{M}$ dNTPs and 0.5 Units Platinum ${ }^{\circledR}$ Taq DNA Polymerase (Invitrogen ${ }^{T M}{ }_{\text {life }}$ technologies). After an initial denaturation at $94^{\circ} \mathrm{C}$ for 4 minutes, 
amplification was achieved by 30 cycles of $94^{\circ} \mathrm{C}$ for 30 seconds, $55^{\circ} \mathrm{C}$ for 30 seconds and $72^{\circ} \mathrm{C}$ for 1.5 minutes, after which there was an extension time of $72^{\circ} \mathrm{C}$ for 7 minutes.

\subsubsection{PCR synthesis of CLiPs}

Each $50 \mu \mathrm{L}$ reaction contained $400 \mathrm{nM}$ of the phosphorylated forward primer and $400 \mathrm{nM}$ of the biotinylated reverse primer as well as $5 \mathrm{ng}$ of PCR amplified CLiP template. The ThermoPol reaction buffer (New England Biolabs ${ }^{\circledR}$ ) contained $20 \mathrm{mM}$

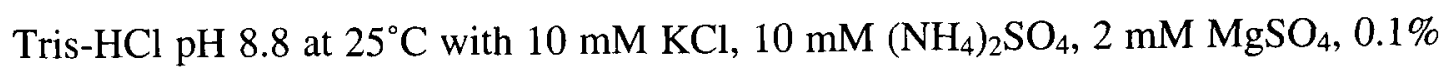
Triton X-100, 6.25 $\mu \mathrm{M}$ modified Fluorescein-12-dUTP (Roche Diagnostics), 43.75 $\mu \mathrm{M}$ dTTP, $50 \mu \mathrm{M}$ each dATP, dGTP, dCTP (giving a final labelling ratio of 1:8) and

2 units of Deep Vent ${ }_{R}^{\circledR}$ (exo-) DNA Polymerase (New England Biolabs). Denaturation and amplification was achieved by 25 cycles of $95^{\circ} \mathrm{C}$ for 30 seconds and $65^{\circ} \mathrm{C}$ for 1 minute, extension was achieved by $72^{\circ} \mathrm{C}$ for 3 minutes. Products were then analysed by electrophoresis using a $2.5 \%$ agarose gel.

\section{$\underline{4.3 ~ P C R ~ p r o d u c t ~ a n a l y s i s ~ b y ~ s u b m a r i n e ~ a g a r o s e ~ g e l ~ e l e c t r o p h o r e s i s ~}$}

Double-stranded PCR products were analysed by electrophoresis using $2.5 \%$ agarose gels containing $320 \mathrm{ng} / \mathrm{mL}$ ethidium bromide, buffered with TAE buffer (40 mM Tris-acetate containing $2 \mathrm{mM} \mathrm{Na} \mathrm{NaDTA}_{2}$. Samples were loaded in buffer containing, $0.25 \%$ bromophenol blue and $30 \%$ glycerol, subjected to gel electrophoresis (typically $124 \mathrm{~V}$ for 30 minutes), and visualised using an ultra violet transilluminator. 


\subsection{Purification of PCR products}

\subsubsection{Agarose gel extraction}

PCR products were extracted from a $2.5 \%$ agarose gel after electrophoresis and purified using the CONCERT $^{\mathrm{TM}}$ Rapid Gel Extraction System (Gibco BRL ${ }^{\circledR}$ ), as per the manufacturers instructions. Briefly, the correct band was excised from the gel and weighed, $60 \mu \mathrm{L}$ of the Gel Solubilisation Buffer [(L1), containing sodium perchlorate, sodium acetate and TBE-solubiliser] was added for every $10 \mathrm{mg}$ of gel and incubated at $50^{\circ} \mathrm{C}$ for 15 minutes or until gel was dissolved. The agarose gel and L1 mixture was added to a spin cartridge and centrifuged at $12000 \mathrm{x}$ g for 1 minute, and the flow-through discarded. To this spin cartridge a further $500 \mu \mathrm{L}$ of $\mathrm{L} 1$ buffer was added, then incubated at room temperature for 1 minute before being centrifuged at $12000 \mathrm{xg}$ for 1 minute and the flow-through again being discarded. $700 \mu \mathrm{L}$ of the Wash Buffer [(L2) containing $\mathrm{NaCl}, \mathrm{Na}_{2} \mathrm{EDTA}$ and Tris- $\mathrm{HCl}$ ] was added to the spin cartridge and incubated at room temperature for 5 minutes before being centrifuged at $12000 \mathrm{x}$ g for 1 minute. The flow-through was discarded and the spin cartridge was centrifuged again at $12000 \mathrm{x}$ g for a further minute to remove residual L2. The spin cartridge was transferred to a new collection tube and $50 \mu \mathrm{L}$ of preheated TE buffer $\left(70^{\circ} \mathrm{C}\right)$ was added before being incubated at room temperature for 1 minute. The purified PCR product was then eluted by centrifugation at $12000 \mathrm{x}$ $\mathrm{g}$ for 2 minutes.

\subsubsection{PCR purification kit}

PCR products were purified using the CONCERT ${ }^{\mathrm{TM}}$ Rapid PCR purification System (Gibco BRL), as per manufacturers instructions. Briefly, an aliquot of TE buffer (containing $10 \mathrm{mM}$ Tris- $\mathrm{HCl} \mathrm{pH} 8.0$ and $1 \mathrm{mM} \mathrm{Na} 2$.EDTA) was preheated at $70^{\circ} \mathrm{C}$ 
before beginning. $400 \mu \mathrm{L}$ of Binding Solution (containing guanidine hydrochloride, $\mathrm{Na}_{2}$.EDTA, Tris- $\mathrm{HCl}$ and isopropanol) was added to the amplification reaction and mixed thoroughly. This was then added to a spin cartridge that was placed inside a 2 $\mathrm{mL}$ wash tube and centrifuged for $1 \mathrm{~min}$ at maximum speed. The flow-through was discarded and $700 \mu \mathrm{L}$ of wash buffer (containing $\mathrm{NaCl}, \mathrm{Na}_{2}$.EDTA, Tris-HCL and ethanol) was added into the spin cartridge. This was centrifuged for 1 minute, the flow-through was discarded and the tube was centrifuged for a further minute to remove all residual wash buffer. The spin cartridge was transferred to a fresh $1.5 \mathrm{~mL}$ recovery tube, and $50 \mu \mathrm{L}$ of the preheated TE buffer was added to elute the DNA from the spin cartridge. This was incubated at room temperature for 1 minute, followed by a 2 minute centrifugation at maximum speed.

\subsection{Single-stranded CLiP synthesis}

\subsubsection{Primer extension}

Single-stranded CLiPs were prepared by amplifying from ds CLiP template using the phosphorylated forward primer (Table 4.0). Each $50 \mu \mathrm{L}$ primer extension amplification contained $400 \mathrm{nM}$ of phosphorylated MOR.B1.Fwd and $4 \mathrm{ng}$ of the ds CLiP template. The reaction buffer was $20 \mathrm{mM}$ Tris- $\mathrm{HCl} \mathrm{pH} 8.8$ containing $10 \mathrm{mM}$ $\mathrm{KCl}, 10 \mathrm{mM}\left(\mathrm{NH}_{4}\right)_{2} \mathrm{SO}_{4}, 2 \mathrm{mM} \mathrm{MgSO}{ }_{4}$ and $0.1 \%$ Triton-X-100, $50 \mu \mathrm{M}$ dNTPs, 5 $\mu \mathrm{M}$ Fluoroscein-12-dUTP (Roche Diagnostics) (giving a labelling ratio of 1:5) and 2 Units of Deep Vent ${ }_{R}^{\circledR}$ (exo-) DNA Polymerase (New England Biolabs). Linear amplification was achieved with 35 cycles of $95^{\circ} \mathrm{C}$ for 30 seconds and $65^{\circ} \mathrm{C}$ for 1 minute, then $72^{\circ} \mathrm{C}$ for 3 minutes. 


\subsubsection{Affinity capture}

The ds CLiPs were purified by electrophoresis on a $2.5 \%$ agarose gel and the correct size product was excised. The gel slab was solubilised at $50^{\circ} \mathrm{C}$ in 3 volumes of the Gel Solubilisation Buffer [(L1), containing sodium perchlorate, sodium acetate and TBE-solubiliser, from the CONCERT ${ }^{\mathrm{TM}}$ Rapid Gel Extraction System (Gibco $\left.\mathrm{BRL}^{\circledR}\right)$ ], until the agarose was dissolved. $100 \mu \mathrm{L}$ of $\mathrm{DEPC}-\mathrm{H}_{2} \mathrm{O}$ was added to $50 \mu \mathrm{L}$ of $10 \mathrm{mg} / \mathrm{mL}$ streptavidin-coated paramagnetic beads (Roche Diagnostics), which had been washed previously in $1 \mathrm{~mL}$ of TNE buffer $(10 \mathrm{mM}$ Tris- $\mathrm{HCl} \mathrm{pH} 8.4$ containing $500 \mathrm{mM} \mathrm{NaCl}$ and $5 \mathrm{mM} \mathrm{Na} \mathrm{mDTA}_{2}$ ), and mixed by vortexing. The beads and $\mathrm{dd}-\mathrm{H}_{2} \mathrm{O}$ were then added to the solubilised agarose gel slab and mixed for 10 minutes to allow the biotin labelled CLiP to bind. A magnet was used to capture the $\mathrm{CLiP}$ and the dd- $\mathrm{H}_{2} \mathrm{O}$ was pipetted off, the beads were then washed twice with $1 \mathrm{~mL}$ TNE buffer (as above) and once with $1 \mathrm{~mL}$ dd- $\mathrm{H}_{2} \mathrm{O}$, all traces of $\mathrm{ddH}_{2} \mathrm{O}$ were removed. Incubating in $25 \mu \mathrm{L}$ of fresh $25 \mathrm{mM} \mathrm{NaOH}$, for 1 minute at room temperature, eluted the ss CLiP (top strand). The ss CLiP was then desalted into water using a sephadex G-25 spin column (Sigma Aldrich, Section 10), dried in a vacuum centrifuge and resuspended in TE buffer $(10 \mathrm{mM}$ Tris- $\mathrm{HCl} \mathrm{pH} 8.0$ containing $1 \mathrm{mM} \mathrm{Na}{ }_{2}$ EDTA) to prevent CLiP degradation.

\subsection{PCR amplification of target for CLiP ligation}

MOR-1 target was prepared by amplifying from Pvu II digested $\mathrm{pBCKS}^{+}$containing MOR-1, using the appropriate forward and reverse primers (Table 4.0). Each $50 \mu \mathrm{L}$ reaction contained $400 \mathrm{nM}$ of each primer and approximately $5 \mathrm{ng}$ of linearised plasmid clone. The reaction buffer was $20 \mathrm{mM}$ Tris- $\mathrm{HCl} \mathrm{pH} 8.4$ containing $50 \mathrm{mM}$ $\mathrm{KCl}, 200 \mu \mathrm{M}$ dNTPs, $1.5 \mathrm{mM} \mathrm{MgCl}_{2}$ and 0.5 Units of Platinum ${ }^{\circledR}$ Taq DNA 
Polymerase (Invitrogen ${ }^{\mathrm{TM}}$ life technologies). After an initial denaturation of $94^{\circ} \mathrm{C}$ for 3 minutes, 30 thermal cycles of $94^{\circ} \mathrm{C}$ for 30 seconds, $60^{\circ} \mathrm{C}$ for 30 seconds and $72^{\circ} \mathrm{C}$ for 1 minute were performed, followed by a final extension of $72^{\circ} \mathrm{C}$ for 7 minutes.

\subsection{CLiP ligation using Thermus thermophilus (Tth) ligase}

PCR amplified MOR-1 was used as the target for CLiP ligation. Each ligation reaction contained approximately $8 \mathrm{ng}$ of the labelled CLiP $(4 \mu \mathrm{L}$ of the $\mathrm{NaOH}$ eluate) and 0.5 pmol of the target in a $20 \mu \mathrm{L}$ volume. The reaction buffer was 50 $\mathrm{mM}$ Tris- $\mathrm{HCl} \mathrm{pH} 8.5$ containing $10 \mathrm{mM} \mathrm{MgCl}_{2}, 50 \mathrm{mM} \mathrm{KCl}, 10 \mathrm{mM}$ DTT, $1 \mathrm{mM}$ $\mathrm{NAD}^{+}$and 40 Units of $T t h$ ligase. After an initial denaturation of $94^{\circ} \mathrm{C}$ for 5 minutes, hybridisation/ligation was achieved by 10 thermal cycles of $94^{\circ} \mathrm{C}$ for 30 seconds and $55^{\circ} \mathrm{C}$ for 10 minutes.

\subsection{Analysis of labelled PCR products and CLiPs using the ABI PRISM $^{\circledR} 377$ DNA sequencer}

Fluorescently labelled PCR products and CLiPs were separated using an ABI PRISM $^{\circledR} 377$ DNA sequencer utilising a denaturing (urea) $4 \%$ polyacrylamide gel with a $12 \mathrm{~cm}$ "well-to-read" distance. Electrophoresis was performed at 750 volts for 3 hours in $1 \times$ TBE buffer (90 nM Tris-borate, $2 \mathrm{mM} \mathrm{Na} \mathbf{N a}_{2}$.EDTA). $1 \mu \mathrm{L}$ of sample was loaded in $2 \mu \mathrm{L}$ of loading buffer containing 12:2:1 deionised formamide: $50 \mathrm{mg} / \mathrm{mL}$ blue dextran/ $25 \mathrm{mM} \mathrm{Na}_{2}$.EDTA: GENESCAN ${ }^{\circledR}-350$ (TAMRA) size standard or -2500 (ROX) size standard (PE Applied Biosystems). All samples were denatured for 2 minutes at $95^{\circ} \mathrm{C}$, before being snap chilled prior to loading. The resulting file was analysed using GeneScan ${ }^{\circledR}$ Analysis Software version 3.7 . 


\subsection{DNA quantification}

Genomic DNA was quantified by measuring the absorbance in a spectrophotometer at $260 \mathrm{~nm}\left(\mathrm{~A}_{260} \times 50=\mu \mathrm{g} / \mathrm{mL}\right)$. DNA was also quantified using a SYBR Green II assay (refer section 4.16.2). Using dilutions of an oligonucleotide with a known concentration produced the standard curve for this assay.

\subsection{Bacterial cultures}

\subsubsection{Maintenance of bacterial cultures}

$50 \mathrm{~mL}$ of sterilised LB broth (Gibco BRL) containing either $75 \mu \mathrm{g} / \mathrm{mL}$ of ampicillin or $30 \mu \mathrm{g} / \mathrm{mL}$ of chloramphenicol was inoculated with a loop of frozen stock colonies and grown overnight in a shaking incubator at $37^{\circ} \mathrm{C}$ and $230 \mathrm{rpm}$ to produce large quantities of the transformed colonies to be isolated using a miniprep (see 4.10.2). $10 \%$ agar plates were produced by making $500 \mathrm{~mL}$ of $\mathrm{LB}$ broth with $5 \mathrm{~g}$ of agar that was sterilised by autoclaving. The antibiotic of choice was added before pouring plates and blue-white selection was used to determine which colonies contained the MOR-1 gene insert. $40 \mu \mathrm{L}$ of $20 \mu \mathrm{mg} / \mathrm{mL}$ 5-bromo-4-chloro-3-indolyl-beta-Dgalactopyranoside (Xgal) and $8 \mu \mathrm{L}$ of $25 \mathrm{mg} / \mathrm{mL}$ isopropyl thiogalactoside (IPTG) was mixed and spread aseptically onto each $10 \%$ agar plate, this was allowed to dry before the plate was streaked with an inoculated loop of colonies. Blue colonies will form when there are no transformed bacteria (as the $\beta$-galactosidase gene is expressed) and white colonies will form when the bacteria are transformed ( $\beta$ galactosidase gene is inactivated by inserted gene). The plate was incubated at $37^{\circ} \mathrm{C}$ overnight and white colonies were selected. 


\subsubsection{Purification of plasmids using miniprep}

A single colony was used to inoculate $3 \mathrm{ml}$ of LB broth (Gibco BRL), containing a final concentration of $75 \mu \mathrm{g} / \mathrm{mL}$ ampicillin. The inoculated broth was grown overnight in a shaking incubator at $37^{\circ} \mathrm{C}$ and $230 \mathrm{rpm} .3 \mathrm{~mL}$ of cells were centrifuged at $15300 \mathrm{x} \mathrm{g}$ for 30 seconds at room temperature, the supernatant was discarded and the tubes were inverted to remove all traces of growth broth. The pellet was resuspended in $200 \mu \mathrm{L}$ of $10 \mathrm{mM}$ Tris- $\mathrm{HCl} \mathrm{pH} 8.0$ containing $10 \mathrm{mM}$ $\mathrm{Na}_{2}$ EDTA and $25 \mu \mathrm{g} / \mathrm{mL}$ RNase $\mathrm{A}$, then vortexed for 2 minutes to ensure thorough resuspension. $200 \mu \mathrm{L}$ of $0.2 \mathrm{M} \mathrm{NaOH}$ containing $1 \% \mathrm{w} / \mathrm{v}$ SDS was added and the tube was gently inverted several times to lyse bacterial cell wall, before being incubated for 5 minutes at room temperature. $200 \mu \mathrm{L}$ of $3 \mathrm{M}$ potassium acetate $\mathrm{pH}$ 6.0 was added to precipitate the genomic DNA, the tube was then inverted and centrifuged at room temperature for 5 minutes at $15300 \mathrm{x}$ g. The supernatant was removed and 0.7 volumes of isopropanol was added, mixed and left to incubate at room temperature for 5 minutes. The DNA was pelleted by centrifugation at $15300 \mathrm{x}$ $\mathrm{g}$ for 10 minutes and the isopropanol supernatant was removed. $200 \mu \mathrm{L}$ of ice-cold $70 \% \mathrm{EtOH}$ was then added to remove any remaining salts, the tube was centrifuged for 3 minutes at $12000 \mathrm{rpm}$ then inverted to decant excess EtOH, pellet was allowed to air dry before being resuspended in $100 \mu \mathrm{L}$ of TE buffer $(10 \mathrm{mM}$ Tris- $\mathrm{HCl} \mathrm{pH} 8.0$ containing $1 \mathrm{mM} \mathrm{Na}{ }_{2}$ EDTA).

Further purification is required if the DNA is to be of a suitable quality for DNA sequencing or probe synthesis, this was achieved by phenol chloroform extraction.

Briefly, an equal volume of phenol/chloroform was added then vortexed until an emulsion formed, the tube was centrifuged at $15300 \mathrm{x} \mathrm{g}$ for 5 minutes at room 
temperature and the aqueous phase was retained. To remove any traces of proteins, equal volumes of chloroform/isoamyl alcohol (24:1) were added and the tube mixed briefly before being centrifuged at $15300 \mathrm{x}$ g for 5 minutes. The upper aqueous phase was removed and the amount estimated, to this 0.1 volumes of $3 \mathrm{M} \mathrm{NaAc} \mathrm{pH}$ 5.0 and 2.75 volumes of ice-cold $100 \% \mathrm{EtOH}$ were added and incubated at $-20^{\circ} \mathrm{C}$ for 30 minutes. The DNA was pelleted by centrifugation at $4^{\circ} \mathrm{C}$ for 10 minutes at 15300 $\mathrm{x} \mathrm{g}$ and the pellet washed with $200 \mu \mathrm{L}$ of ice-cold $70 \% \mathrm{EtOH}$ and centrifuged as before for 5 minutes. The tube was inverted and air-dried to remove all traces of $\mathrm{EtOH}$, the pellet was resuspended by vortexing vigorously in $50 \mu \mathrm{L}$ of TE buffer $(10$ mM Tris- $\mathrm{HCl}$ pH 8.0 containing $1 \mathrm{mM} \mathrm{Na} 2$ EDTA).

\subsubsection{Stocking and restoring plasmids}

A final concentration of $15 \%$ sterile glycerol was added to the cloned plasmid vector giving a final volume of $1 \mathrm{~mL}$, this was frozen down in cryotubes at $-80^{\circ} \mathrm{C}$. Plasmids were restored by scraping an inoculation loop over the top of the frozen stock without allowing sample to thaw. The loop was then spread over an agar plate and incubated at $37^{\circ} \mathrm{C}$ overnight.

\subsection{Sephadex G-25 spin columns}

Sephadex G-25 (Sigma Aldrich $^{\circledR}$ ) spin columns were used for the removal of salts and purification of ss CLiPs and sequencing reaction. A general purpose spin column was placed in a $1.5 \mathrm{~mL}$ microcentrifuge tube and prepared by adding $700 \mu \mathrm{L}$ of sephadex G-25 resin resuspended in destilled, deionised- $\mathrm{H}_{2} \mathrm{O}\left(\mathrm{dd}-\mathrm{H}_{2} \mathrm{O}\right)$, and centrifuged at $420 \mathrm{x}$ g for 1 minute. $50 \mu \mathrm{L}$ of $\mathrm{ddH}_{2} \mathrm{O}$ was added to the spin column and centrifuged again at $420 \mathrm{x}$ g for 1 minute, the sephadex G-25 spin column was 
then transferred to a new $1.5 \mathrm{~mL}$ microcentrifuge tube, before the addition of no more than $50 \mu \mathrm{L}$ of the sample to be purified. This was centrifuged at $420 \times \mathrm{g}$ for 1 minute and the desalted product, if required, was spun in a vacuum centrifuge at $45^{\circ} \mathrm{C}$ until dried.

\subsection{Generation of riboprobe template using overlapping oligonucleotides (oligos)}

\subsubsection{Generation of ds DNA template for exon 4 and 9 riboprobe synthesis using overlapping oligos}

Overlapping oligonucleotides were used to generate a riboprobe template by PCR fill-in. Each 50.0 $\mu \mathrm{L}$ PCR amplification contained $1 \mu \mathrm{M}$ of each oligonucleotide. The reaction buffer was $20 \mathrm{mM}$ Tris- $\mathrm{HCl}(\mathrm{pH} 8.4$ ) containing $50 \mathrm{mM} \mathrm{KCl}, 2.5 \mathrm{mM}$ $\mathrm{MgCl}_{2}, 200 \mu \mathrm{M} \mathrm{dNTPs}$ and 2 Units Platinum $^{\circledR}$ Taq DNA Polymerase (Invitrogen $^{\mathrm{TM}}$ life technologies). An initial denaturation and amplification was achieved with 5 cycles of $95^{\circ} \mathrm{C}$ for 30 seconds and $60^{\circ} \mathrm{C}$ for 10 seconds. A secondary denaturation, then extension was achieved by 20 cycles of $95^{\circ} \mathrm{C}$ for 30 seconds and $72^{\circ} \mathrm{C}$ for 5 seconds.

\subsubsection{Generation of exon 4 and 9 riboprobe templates using second round PCR fill-in}

Templates for riboprobe synthesis were prepared by second round PCR off the exon template (see above). Each $50 \mu \mathrm{L}$ PCR amplification contained $200 \mathrm{nM}$ of appropriate forward and reverse primer pairs and $1 \mathrm{ng}$ of PCR generated exon template. The reaction buffer was $20 \mathrm{mM}$ Tris- $\mathrm{HCl}$ ( $\mathrm{pH} 8.4$ ) containing $50 \mathrm{mM} \mathrm{KCl}$, $2.5 \mathrm{mM} \mathrm{MgCl}_{2}, 200 \mu \mathrm{M}$ dNTPs and 2 Units Platinum $^{\circledR}$ Taq DNA Polymerase (Invitrogen ${ }^{\mathrm{TM}}$ life technologies). The ds DNA template for exon 4 riboprobe synthesis was 
generated by 30 cycles of $94^{\circ} \mathrm{C}$ for 30 seconds and $55^{\circ} \mathrm{C}$ for 15 seconds. The exon 9 ds DNA template was generated by 5 cycles of $95^{\circ} \mathrm{C}$ for 30 seconds and $55^{\circ} \mathrm{C}$ for 10 seconds, followed by 20 cycles of $95^{\circ} \mathrm{C}$ for 30 seconds and $72^{\circ} \mathrm{C}$ for 5 seconds.

\subsubsection{Generation of ds DNA template for riboprobe synthesis using the Klenow fragment}

Overlapping oligonucleotides were used to generate a riboprobe template using the Klenow enzyme, which is the large fragment of DNA polymerase I that has been treated with subtilisin to inactivate DNA polymerase I $5^{\prime}=>3^{\prime}$ exonuclease activity. Each $30.0 \mu \mathrm{L}$ amplification contained $5 \mu \mathrm{M}$ of each oligonucleotide. The reaction buffer was $20 \mathrm{mM}$ Tris- $\mathrm{HCl}(\mathrm{pH} 8.4)$ containing $50 \mathrm{mM} \mathrm{KCl}, 10 \mathrm{mM} \mathrm{MgCl} 2,200$ $\mu \mathrm{M} d \mathrm{dTP}$ s and 2 Units of Klenow enzyme (Invitrogen ${ }^{\mathrm{TM}}$ life technologies). After an initial denaturation of $95^{\circ} \mathrm{C}$ for 2 minutes, there was a slow ramp down to $37^{\circ} \mathrm{C}$ over 10 minutes, at $37^{\circ} \mathrm{C} 2$ Units of the Klenow enzyme was added and this was held at $37^{\circ} \mathrm{C}$ for 30 minutes for extension and then $72^{\circ} \mathrm{C}$ for 2 minutes before being snap chilled on ice.

\subsubsection{Polishing of 3'-ends using T4 DNA polymerase}

T4 DNA polymerase (Roche Diagnostics) was used to remove any non-templated adenines added to PCR products by the action of PLATINUM ${ }^{\circledR}$ Taq DNA polymerase (Invitrogen ${ }^{\top M}$ life technologies). After PCR amplification each sample was heated to $99^{\circ} \mathrm{C}$ for 10 minutes to inactivate the PLATINUM ${ }^{\circledR}$ Taq DNA polymerase. The samples were cooled to room temperature before 0.5 Units of T4 DNA polymerase was added. The samples were then heated to $37^{\circ} \mathrm{C}$ for 5 minutes before 
being heated to $75^{\circ} \mathrm{C}$ for 10 minutes to inactivate the T4 DNA polymerase. This removed any single-stranded overhangs before riboprobe synthesis.

\subsection{Generation of riboprobe template using PCR and restriction digestion}

\subsubsection{Restriction digestion of the mammalian GATEWAY pDEST $^{\text {TM }} 12.2$ vector containing MOR-1}

E.coli cells transformed with the mammalian GATEWAY $\mathrm{pDEST}^{\mathrm{TM}} 12.2$ vector (Invitrogen ${ }^{\text {TM }}$ life technologies) containing MOR-1 was kindly gifted by Bronwyn Kivell. 1 $\mu \mathrm{g}$ of the this vector was digested with 15 Units of Hind III $(10 \mathrm{U} / \mu \mathrm{L}$, Roche Diagnostics) in $10 \mathrm{mM}$ Tris- $\mathrm{HCl} \mathrm{pH} 7.5$ containing $10 \mathrm{mM} \mathrm{MgCl} 2,50 \mathrm{mM} \mathrm{NaCl}, 1$ $\mathrm{mM}$ 1,4-Dithioerythritol (DTE) for 4 hours at $37^{\circ} \mathrm{C}$. Heating to $65^{\circ} \mathrm{C}$ for 15 minutes inactivated the restriction endonuclease.

\subsubsection{PCR synthesis of exon 3 riboprobe template}

Template for the exon 3 riboprobe was prepared by PCR amplification with the correct forward and reverse primers to the MOR-1 gene cloned into the Mammalian GATEWAY $\operatorname{pDEST}^{\mathrm{rM}} 12.2$ vector. A $50 \mu \mathrm{L}$ PCR amplification contained approximately $5 \mathrm{ng}$ of the linearised GATEWAY vector with $200 \mathrm{nM}$ each of MOR.Ex3.primer.Fwd and MOR.Ex3.T7.primer.Rev. The reaction buffer was 20 $\mathrm{mM}$ Tris- $\mathrm{HCl}\left(\mathrm{pH} 8.4\right.$ ) containing $50 \mathrm{mM} \mathrm{KCl}, 1.5 \mathrm{mM} \mathrm{MgCl}_{2}, 200 \mu \mathrm{M} \mathrm{dNTPs}$ and

2 Units Platinum ${ }^{\circledR}$ Taq DNA Polymerase (Invitrogen ${ }^{\top M}$ life technologies). After an initial denaturation at $94^{\circ} \mathrm{C}$ for 4 minutes, amplification was achieved by 30 cycles of $95^{\circ} \mathrm{C}$ for 30 seconds, $50^{\circ} \mathrm{C}$ for 30 seconds and $72^{\circ} \mathrm{C}$ for 1 minute and 30 seconds. This was followed by a final extension at $72^{\circ} \mathrm{C}$ for 7 minutes and a $4^{\circ} \mathrm{C}$ hold. 


\subsubsection{PCR synthesis of MOR 287 bp fragment}

A $50 \mu \mathrm{L}$ PCR amplification contained approximately $5 \mathrm{ng}$ of the linearised GATEWAY vector (4.13.1), with $400 \mathrm{nM}$ each of the MOR.794.Fwd (Table 4.0) and MOR.287.T7.Rev primers (Table 4.1). The reaction buffer was $20 \mathrm{mM}$ Tris- $\mathrm{HCl}$ (pH 8.4) containing $50 \mathrm{mM} \mathrm{KCl}, 2.5 \mathrm{mM} \mathrm{MgCl} 2,200 \mu \mathrm{M}$ dNTPs and 4 Units Platinum $^{\circledR}$ Taq DNA Polymerase (Invitrogen ${ }^{\top M}$ life technologes). An initial denaturation was achieved by heating to $95^{\circ} \mathrm{C}$ for 5 minutes. Further denaturation and amplification was achieved with 25 cycles of $94^{\circ} \mathrm{C}$ for 30 seconds, $55^{\circ} \mathrm{C}$ for 30 seconds and $72^{\circ} \mathrm{C}$ for 30 seconds, followed by $72^{\circ} \mathrm{C}$ for 7 minutes.

\subsubsection{Restriction digestion of MOR $287 \mathrm{bp}$ PCR product to generate the template for exon 1 and $P v u$ II riboprobe synthesis}

The MOR 287 bp PCR product (4.13.3) was restriction digested either with Pvu II (Invitrogen ${ }^{\top M}$ life technologies) to generate a 222 bp T7-tailed fragment and a $75 \mathrm{bp}$ fragment or with Hae III (Boehringer Mannheim) to generate a $117 \mathrm{bp}$ T7-tailed fragment and a $170 \mathrm{bp}$ fragment. The $P v u I I$ generated $222 \mathrm{bp}$ fragment was used to synthesise the riboprobe to the 5' UTR + exon 1 of Oprm and the Hae III generate 117 bp fragment was used to produce the exon 1 riboprobe (refer 4.14). The MOR 287 PCR product was purified by ethanol precipitation (refer 4.15 ) and resuspended in $2 \mathrm{x}$ T3/T7 transcription buffer $(80 \mathrm{mM}$ Tris- $\mathrm{HCl} \mathrm{pH} 8.0$ containing $16 \mathrm{mM} \mathrm{MgCl}$, $4 \mathrm{mM}$ spermidine- $(\mathrm{HCl})_{3}$ and $50 \mathrm{mM} \mathrm{NaCl}$, Invitrogen ${ }^{\mathrm{TM}}$ life technologies $)$ Both digestions contained approximately $2 \mu \mathrm{g}$ of the purified PCR product with 10 Units of the restriction enzyme in $0.8 \times \mathrm{T} 3 / \mathrm{T} \&$ transcription buffer $(32 \mathrm{mM}$ Tris- $\mathrm{HCl} \mathrm{pH}$ 8.0 containing $6.4 \mathrm{mM} \mathrm{MgCl}, 1.6 \mathrm{mM}$ spermidine- $(\mathrm{HCl})_{3}$ and $20 \mathrm{mM} \mathrm{NaCl}$, 
Invitrogen ${ }^{\mathrm{TM}}$ life technologies). The restriction digestion was incubated at $37^{\circ} \mathrm{C}$ for 2 hours, thereafter adding a final concentration of $10 \mathrm{mM} \mathrm{Na}$. EDTA inactivated both restriction enzymes.

\subsection{Riboprobe synthesis via in vitro transcription using T7 RNA polymerase}

Each $30 \mu \mathrm{L}$ riboprobe reaction contained approximately $50 \mathrm{ng}$ of purified PCR template $(4.5 \mu \mathrm{L}$ of pellet resuspended in $2 \times \mathrm{T} 3 / \mathrm{T} 7$ Buffer), $0.5 \mathrm{mM}$ each of CTP, GTP and ATP, 0.4 mM UTP, 0.1 mM Cy3-UTP (Amersham Pharmacia Biotech giving a final labelling ratio of 1:5), $5 \mu \mathrm{M}$ DTT, 40 Units RNaseOUT Recombinant Ribonuclease Inhibitor (Invitrogen ${ }^{\mathrm{TM}}$ life technologies), 100 Units T7 RNA Polymerase (Invitrogen ${ }^{\mathrm{TM}}$ life technologies) and additional $5 \mathrm{x}$ T3/T7 Buffer $(0.2 \mathrm{M}$ Tris- $\mathrm{HCl} \mathrm{pH} 8.0$ containing $40 \mathrm{mM} \mathrm{MgCl}, 10 \mathrm{mM}$ spermidine- $(\mathrm{HCl})_{3}$ and $125 \mathrm{mM} \mathrm{NaCl}$ ) to give a final $1 \mathrm{x}$ concentration. Each tube was incubated at $37^{\circ} \mathrm{C}$ for at least 4 hours and briefly centrifuged every 30 minutes to reduce condensation, 124.5 Units of DNase I was then added to each tube and then incubated for a further 15 minutes at $37^{\circ} \mathrm{C}$.

Fluorescein-12-UTP -labelled riboprobes were synthesised using $0.5 \mathrm{mM}$ each CTP, GTP, TTP, $0.222 \mathrm{mM}$ UTP and 0.278 mM Fluorescein-12-UTP (Roche Diagnostics) giving a final labelling ratio of $1: 1.8 .^{2}$

\footnotetext{
${ }^{2}$ NB: Fluorescein-12-UTP-labelled riboprobes are referred to as FITC-labelled riboprobes throughout this project.
} 


\subsection{Desalting riboprobes and purifying small PCR products by ethanol precipitation}

Riboprobes were desalted and PCR products were purified using ethanol precipitation with linear poly-acrylamide as the neutral carrier (Gaillard and Strauss 1990). Briefly, the excess nucleic acids and primers were removed by the addition of 0.1 volumes of $3 \mathrm{M} \mathrm{NaOAc} \mathrm{pH} 5.2,1 \mu \mathrm{g}$ linear acrylamide and 2.75 volumes of $100 \%$ ethanol. The tube was inverted several times and incubated at $80^{\circ} \mathrm{C}$ for 30 minutes, the DNA was pelleted by centrifugation at $15300 \mathrm{x}$ for 10 minutes at $4^{\circ} \mathrm{C}$ in a Sorvall centrifuge and the supernatant was discarded. The pellet was washed with $200 \mu \mathrm{L}$ of $70 \%$ ethanol and centrifuged as before for 5 minutes, the supernatant was discarded and the pellet left to air-dry. This pellet was then resuspended in $20 \mu \mathrm{L}$ of 2x T3/T7 Buffer $\left[0.2 \mathrm{M}\right.$ Tris- $\mathrm{HCl} \mathrm{pH} 8.0$ containing $40 \mathrm{mM} \mathrm{MgCl}_{2}, 10 \mathrm{mM}$ spermidine- $(\mathrm{HCl})_{3}$ and $125 \mathrm{mM} \mathrm{NaCl}$, Invitrogen ${ }^{\mathrm{TM}}$ life technologres].

\section{$\underline{\text { 4.16 Product analysis }}$}

\subsubsection{Sequencing}

Products for sequencing were purified using ethanol precipitation with linear acrylamide a neutral carrier (refer 4.15) and resuspended in TE buffer (containing 10 $\mathrm{mM}$ Tris- $\mathrm{HCl} \mathrm{pH} 8.0$ and $1 \mathrm{mM} \mathrm{Na}$.EDTA). Sequencing reactions were performed using a ABI PRISM ${ }^{\circledR}$ BigDye $^{\mathrm{TM}}$ Terminator v3.0 Ready Reaction Cycle Sequencing Kit (PE Applied Biosystems). Approximately $25 \mathrm{ng}$ of ds template was added to 4 $\mu \mathrm{L}$ of Terminator Ready Reaction mix with $3.2 \mathrm{pmol}$ of primer in a final volume of $10 \mu \mathrm{L} .25$ cycles of $96^{\circ} \mathrm{C}$ for 10 seconds, $50^{\circ} \mathrm{C}$ for 5 seconds and $60^{\circ} \mathrm{C}$ for 4 minutes were performed and the resulting mix was purified by ethanol precipitation (refer 4.15). The purified pellet was resuspended in a 5:1 ratio of deionised formamide: 50 
$\mathrm{mg} / \mathrm{mL}$ blue dextran/ $25 \mathrm{mM} \mathrm{Na}$.EDTA. Sequenced product was analysed using a $4 \%$ polyacrylamide gel electrophoresis (PAGE) $48 \mathrm{~cm}$ well-to-read plate on the $\mathrm{ABI}$ PRISM $^{\circledR} 377$ DNA Sequencer. The resulting file was analysed using GeneScan ${ }^{\circledR}$ Analysis Software version 3.7.

\subsubsection{Size and quality analysis of fluorescently labelled riboprobes}

The size, yield and quality of fluorescently labelled riboprobes were determined using an ABI PRISM ${ }^{\circledR} 377$ DNA sequencer. These products were separated utilising a denaturing (urea) $4 \%$ polyacrylamide gel with a $12 \mathrm{~cm}$ "well-to-read" distance. Products were purified by ethanol precipitation and diluted according to which fluorophore they were labelled with (e.g. FITC-labelled riboprobes were diluted 1000-fold and CY3-labelled riboprobes were diluted $50-100$-fold). $1 \mu \mathrm{L}$ of riboprobe was added to $0.5 \mu \mathrm{L}$ of GeneScan ${ }^{\mathrm{TM}}-350$ ROX size standard (Applied Biosystems) and $2 \mu \mathrm{L}$ of the loading buffer, containing a $1: 6$ ratio of $50 \mathrm{mg} / \mathrm{mL}$ blue dextran/ $25 \mathrm{mM} \mathrm{Na}_{2}$.EDTA: 100\% deionised formamide. Samples were heated to $95^{\circ} \mathrm{C}$ for 2 minutes and then snap chilled before being loaded. The resulting file was analysed using GeneScan ${ }^{\circledR}$ Analysis software version 3.7.

\subsubsection{Product quantification using a SYBR Green II assay}

Labelled CLiPs and riboprobes were quantified with a Molecular Devices SpectraMAX Gemini using 1.1x SYBR Green II (Molecular Probes) as the fluorescent dye for the analysis of single-stranded products. For DNA analysis (CLiPs) a diluted oligonucleotide was used as the standards for single-stranded quantification (diluted from $50 \mathrm{ng} / \mu \mathrm{L}$ to $50 \mathrm{pg} / \mu \mathrm{L}$ ). For RNA analysis (riboprobes) diluted yeast t-RNA was used as the standards for single-stranded product 
quantification (diluted from $50 \mathrm{ng} / \mu \mathrm{L}$ to $100 \mathrm{pg} / \mu \mathrm{L}$ ). In each case the products were diluted by serial dilution in a Nunc black 36 well microtitre plate (Nunc) using a volume of $200 \mu \mathrm{L}$ for each sample. Typically 10-, 50-, 100-, and 200-fold dilutions were performed for each sample and blanks were included. Excitation was set at 497 $\mathrm{nm}$ and emission at $520 \mathrm{~nm}$, with a cut-off of $515 \mathrm{~nm}$, and 30 reads per well. Readings from the standard samples are used to generate a standard curve. This standard curve is used to estimate product concentration.

\begin{tabular}{|c|c|c|c|c|c|c|c|c|c|c|}
\hline & \multicolumn{9}{|c|}{ Plate\#1 } & \multirow{3}{*}{ Endooint } \\
\hline & 2 & 3 & 4 & 5 & 6 & 8 & 9 & 10 & 11 & \\
\hline \multicolumn{10}{|c|}{ A } & \\
\hline 3 & \multicolumn{7}{|c|}{1322.2329 .88147 .14603023291019 .07124 .4489 .664} & & 0.000 & Fluorescence \\
\hline c & \multicolumn{4}{|c|}{199.2359 .03618 .30214 .107} & \multicolumn{4}{|c|}{162.785 .6642 .43811 .881} & -10.85 & \multirow{2}{*}{\begin{tabular}{|l} 
Ex Em Cutoff \\
Lm1497 520515 \\
"Aüto Cütof
\end{tabular}} \\
\hline D) & \multicolumn{4}{|c|}{269.04105 .9238 .14110 .702} & \multicolumn{2}{|c|}{282.2536 .18} & 33.125 & 5.377 & \multirow{2}{*}{$\begin{array}{l}-15.50 \\
0.931\end{array}$} & \\
\hline E) & \multicolumn{4}{|c|}{303.5275 .98340 .8268 .386} & \multicolumn{2}{|c|}{198.1419 .79} & $4-1.4091$ & 15327 & & \multirow{3}{*}{$\begin{array}{l}\text { Automix: Once } \\
\text { Calibrate: On } \\
\text { PMT: High } \\
\end{array}$} \\
\hline$F$ & \multicolumn{4}{|c|}{1.482 .2447 .41106 .9663 .778} & & \multirow{2}{*}{156722049} & 132405 & 99934 & & \\
\hline G & \multicolumn{4}{|c|}{36595742.93226 .49164 .76} & 2030 & & 2105.31 & 176.08 & & \\
\hline$H$ & \multicolumn{4}{|c|}{377.5074 .05215 .94023 .670} & & 29.3787 .256 & 2.816 & & & \multirow[t]{2}{*}{ Reacs $M$ Nell: 30} \\
\hline \multirow{2}{*}{\multicolumn{10}{|c|}{$\begin{array}{l}\text { remperature Set Point: } 37.0 \text { Mean } 37.0 \\
\text { Wavelength Combination: ILm1 }\end{array}$}} & \\
\hline & & & $1=$ & 715 & & & & & & $\begin{array}{l}\text { Plate Last Read } \\
1643 \quad 17 / 02 / 03\end{array}$ \\
\hline
\end{tabular}

Figure 4.0a Plate reading from a SYBR Green II assay. The area shown in blue shows the readings used to generate the standard curve (Figure 4.1), the area shown in pink represents the readings produced by serial dilutions of the riboprobes. The area shown in yellow are the readings produced by blank samples (i.e. with no probe) and white cell represents a blank sample of TE buffer. Figure 4.0b shows what sample loaded in each lane and the dilution.

An approximation of riboprobe concentration was calculated using the standard curve (Figure 4.1) and the dilution factor. An example is outlined below:

\section{Sample calculation of CY3-labelled exon 3 riboprobe concentration:}

Figure 4.0a lane C2 reports a reading of 199.23 for a neat sample of a CY3-labelled exon 3 riboprobe. From the standard curve (Figure 4.1) a mean value of 199.23 
approximately corresponds to a concentration of $120 \mathrm{ng}$ per $20 \mu \mathrm{L}$ of sample used in this assay. Therefore the concentration of the CY-3 labelled exon 3 riboprobe was 6 $\mathrm{ng} / \mu \mathrm{L}$.

\begin{tabular}{|c|c|c|c|c|c|c|c|c|c|c|}
\hline Al & 2 & 3 & 4 & 5 & 6 & 7 & 8 & 9 & 10 & 11 \\
\hline $\bar{B}$ & $10 \frac{\text { STD }}{\mu \mathrm{g}}$ & $125 \frac{\text { STD }}{\mathrm{ng}}$ & $62.5 \frac{\text { STD }}{\mathrm{ng}}$ & $\underset{31.25 \mathrm{ng}}{\mathbf{S T D}}$ & $\frac{\text { STD }}{15.6 \mathrm{ng}}$ & $7.81^{\frac{\text { STD }}{\mathrm{ng}}}$ & $3.91 \frac{\text { STD }}{\mathrm{ng}}$ & $196 \frac{\text { STD }}{\mathrm{ng}}$ & & $\frac{\text { BLANK }}{\text { TE only }}$ \\
\hline $\bar{C}$ & $\begin{array}{l}\text { Ex3- } \\
\text { CY3 } \\
\text { Neat }\end{array}$ & $\begin{array}{l}\text { Ex3- } \\
\text { CY3 } \\
\text { 10-fold } \\
\text { dilution } \\
\end{array}$ & $\begin{array}{l}\text { Ex3- } \\
\text { CY3 } \\
100 \text {-fold } \\
\text { dilution }\end{array}$ & $\begin{array}{l}\text { Ex3- } \\
\text { CY3 } \\
\text { 200-fold } \\
\text { dilution }\end{array}$ & & $\begin{array}{l}\text { Ex3- } \\
\text { CY3 } \\
\text { 10-fold } \\
\text { dilution } \\
\end{array}$ & $\begin{array}{l}\text { Ex3- } \\
\text { CY3 } \\
\text { 10-fold } \\
\text { dilution }\end{array}$ & $\begin{array}{l}\text { Ex3- } \\
\text { CY3 } \\
\text { 100-fold } \\
\text { dilution } \\
\end{array}$ & $\begin{array}{l}\text { Ex3- } \\
\text { CY3 } \\
200 \text {-fold } \\
\text { dilution }\end{array}$ & $\frac{\text { BLANK }}{\text { No probe }}$ \\
\hline $\mathrm{D}$ & $\begin{array}{l}\text { Ex4- } \\
\text { CY3 } \\
\text { Neat }\end{array}$ & $\begin{array}{l}\text { Ex4- } \\
\text { CY3 } \\
\text { 10-fold } \\
\text { dilution } \\
\end{array}$ & $\begin{array}{l}\text { Ex4- } \\
\text { CY3 } \\
\text { 50-fold } \\
\text { dilution } \\
\end{array}$ & $\begin{array}{l}\text { Ex4- } \\
\text { CY3 } \\
\text { 100-fold } \\
\text { dilution }\end{array}$ & & $\begin{array}{l}\text { Ex4- } \\
\text { CY3 } \\
\text { Neat }\end{array}$ & $\begin{array}{l}\text { Ex4- } \\
\text { CY3 } \\
\text { 10-fold } \\
\text { dilution }\end{array}$ & $\begin{array}{l}\text { Ex4- } \\
\text { CY3 } \\
\text { 50-fold } \\
\text { dilution } \\
\end{array}$ & $\begin{array}{l}\text { Ex4- } \\
\text { CY3 } \\
100 \text {-fold } \\
\text { dilution } \\
\end{array}$ & $\frac{\text { BLANK }}{\text { No probe }}$ \\
\hline $\mathrm{E}$ & $\begin{array}{l}\text { Ex9- } \\
\text { CY3 } \\
\text { Neat }\end{array}$ & $\begin{array}{l}\text { Ex9- } \\
\text { CY3 } \\
\text { 10-fold } \\
\text { dilution }\end{array}$ & $\begin{array}{l}\text { Ex9- } \\
\text { CY3 } \\
\text { 50-fold } \\
\text { dilution }\end{array}$ & $\begin{array}{l}\text { Ex9- } \\
\text { CY3 } \\
\text { 100-fold } \\
\text { dilution }\end{array}$ & & $\begin{array}{l}\text { Ex9- } \\
\text { CY3 } \\
\text { Neat }\end{array}$ & $\begin{array}{l}\text { Ex9- } \\
\text { CY3 } \\
\text { 10-fold } \\
\text { dilution }\end{array}$ & $\begin{array}{l}\text { Ex9- } \\
\text { CY3 } \\
\text { 50-fold } \\
\text { dilution }\end{array}$ & $\begin{array}{l}\text { Ex9- } \\
\text { CY3 } \\
\text { 100-fold } \\
\text { dilution }\end{array}$ & $\frac{\text { BLANK }}{\text { No probe }}$ \\
\hline$F$ & $\begin{array}{l}\text { Ex3- } \\
\text { FITC } \\
\text { Neat }\end{array}$ & $\begin{array}{l}\text { Ex3- } \\
\text { FITC } \\
\text { 10-fold } \\
\text { dilution }\end{array}$ & $\begin{array}{l}\text { Ex3- } \\
\text { FITC } \\
\text { 50-fold } \\
\text { dilution }\end{array}$ & $\begin{array}{l}\text { Ex3- } \\
\text { FITC } \\
100 \text {-fold } \\
\text { dilution }\end{array}$ & & $\begin{array}{l}\text { Ex3- } \\
\text { FITC } \\
\text { Neat }\end{array}$ & $\begin{array}{l}\text { Ex3- } \\
\text { FITC } \\
\text { 10-fold } \\
\text { dilution } \\
\end{array}$ & $\begin{array}{l}\text { Ex3- } \\
\text { FITC } \\
\text { 50-fold } \\
\text { dilution } \\
\end{array}$ & $\begin{array}{l}\text { Ex3- } \\
\text { FITC } \\
\text { 100-fold } \\
\text { dilution } \\
\end{array}$ & \\
\hline $\bar{G}$ & $\begin{array}{l}\text { Ex9- } \\
\text { FITC } \\
\text { Neat }\end{array}$ & $\begin{array}{l}\text { Ex9- } \\
\text { FITC } \\
\text { 10-fold } \\
\text { dilution }\end{array}$ & $\begin{array}{l}\text { Ex9- } \\
\text { FITC } \\
\text { 50-fold } \\
\text { dilution } \\
\end{array}$ & $\begin{array}{l}\text { Ex9- } \\
\text { FITC } \\
\text { l00-fold } \\
\text { dilution } \\
\end{array}$ & & $\begin{array}{l}\text { Ex9- } \\
\text { FITC } \\
\text { Neat }\end{array}$ & $\begin{array}{l}\text { Ex9- } \\
\text { FITC } \\
\text { 10-fold } \\
\text { dilution }\end{array}$ & $\begin{array}{l}\text { Ex9- } \\
\text { FITC } \\
\text { s0-fold } \\
\text { dilution } \\
\end{array}$ & $\begin{array}{l}\text { Ex9- } \\
\text { FITC } \\
\text { 100-fold } \\
\text { dilution }\end{array}$ & \\
\hline $\mathrm{H}$ & $\begin{array}{l}\text { 287- } \\
\text { FITC } \\
\text { Neat }\end{array}$ & $\begin{array}{l}287- \\
\text { FITC } \\
\text { 10-fold } \\
\text { dilution } \\
\end{array}$ & $\begin{array}{l}287- \\
\text { FITC } \\
50 \text {-fold } \\
\text { dilution } \\
\end{array}$ & $\begin{array}{l}287- \\
\text { FITC } \\
100 \text {-fold } \\
\text { dilution } \\
\end{array}$ & & $\begin{array}{l}287- \\
\text { FITC } \\
\text { 10-fold } \\
\text { dilution } \\
\end{array}$ & $\begin{array}{l}287- \\
\text { FITC } \\
\text { 50-fold } \\
\text { dilution } \\
\end{array}$ & $\begin{array}{l}287- \\
\text { FITC } \\
\text { 100-fold } \\
\text { dilution } \\
\end{array}$ & & \\
\hline
\end{tabular}

Figure 4.0b Microtitre plate chart. This chart corresponds to Figure 4.0a and shows which sample was added to each lane. STD is the standard; yeast t-RNA was diluted as above to generate the standard curve. Ex3, Ex4, Ex9 represent riboprobes produced to the exons 3,4 and 9 of the Oprm, respectively. These riboprobes were labelled with Cyanine 3 (CY3) or fluorescein isothiocyanate (FITC). The $\mathbf{2 8 7}$ riboprobe was generated to the 5' UTR and exon 1 of the Oprm. NB "Neat" refers to an undiluted sample. 


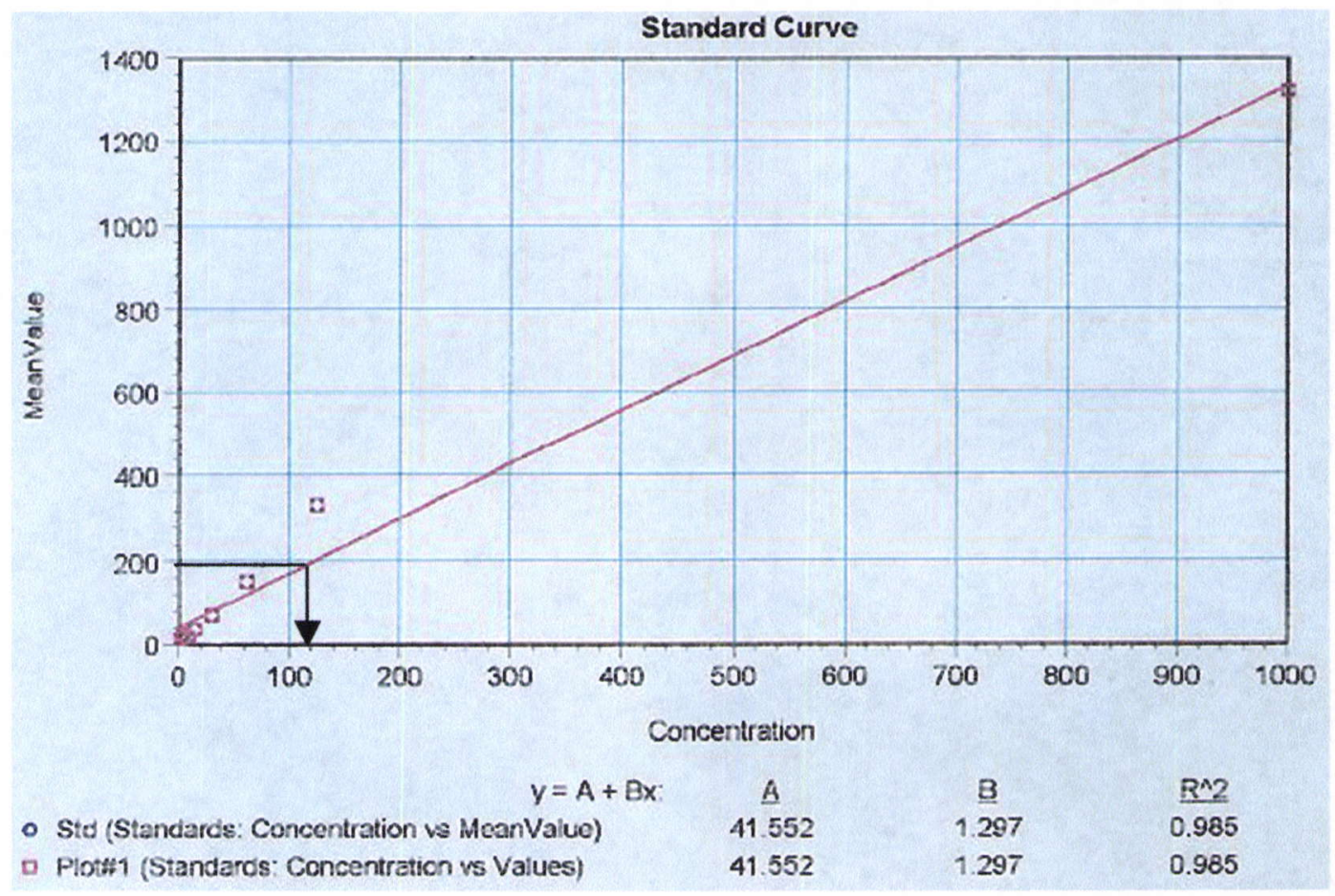

Figure 4.1 Standard curve from a SYBR Green II assay generated by diluting yeast t-RNA. The standard curve was used to estimate the concentrations of each riboprobe to ensure approximately $6 \mathrm{ng}$ of riboprobe was added to each FISH experiment. The arrow depicts the concentration extrapolation of the undiluted CY3labelled exon 3 riboprobe, a mean value of 199.23 gives an approximate concentration of $120 \mathrm{ng}$.

\subsection{Tissue preparation}

\subsubsection{Perfusion protocol and tissue fixation}

Sprague-Dawley rats were given a lethal dose with an intraperitoneal injection of sodium pentobarbital $(100 \mathrm{mg} / \mathrm{kg})$. Animals were perfused by insertion of a needle into the left ventricle and an incision made into the right atrium to allow buffers to circulate [all buffers used on rat brain were RNase free and diethyl pyrocarbonate (DEPC)-treated]. $\quad 400 \mathrm{~mL}$ of cold $0.1 \mathrm{M} \mathrm{PBS,} \mathrm{pH} 7.4$ was perfused through the animal, followed by $500 \mathrm{~mL}$ of fresh $4 \%$ paraformaldehyde in $0.1 \mathrm{M}$ PBS at a rate of $11 \mathrm{~mL} / \mathrm{min}$. Brains were removed from skull and post-fixed for 1.5 hours in $100 \mathrm{~mL}$ of $4 \%$ paraformaldehyde in $0.1 \mathrm{M} \mathrm{PBS}, \mathrm{pH} 7.4$ at $4{ }^{\circ} \mathrm{C}$. After post-fixation, brains 
were cyroprotected by leaving for 1.5 days in $0.1 \mathrm{M}$ PBS containing $30 \%$ sucrose, at $4^{\circ} \mathrm{C}$. Brains were then frozen at $-80^{\circ} \mathrm{C}$ until ready for sectioning.

\subsubsection{Cutting and mounting sections}

Frozen brain was attached in appropriate orientation to cryostat chuck and sections were cut between 10-20 $\mu \mathrm{m}$. These sections were placed in 0.1 M PBS and later floated onto superfrosted, positively charged microscope slides (BDH).

\subsection{Fluorescent in situ hybridisation (FISH)}

\subsubsection{Prehybridisation}

The protocol used was a modification of the Nonradioactive In Situ Hybridisation Application Manual (Roche Molecular Biochemicals 1996). Briefly, mounted sections were removed from the $-80^{\circ} \mathrm{C}$ freezer and dried-out at $40^{\circ} \mathrm{C}$ for 2 hours before the adhesion of a $65 \mu \mathrm{L}$ Gene Frame ${ }^{\circledR}$ (Advanced Biotechnologies Ltd). Sections were incubated for $2 \times 5$ minutes in $0.1 \mathrm{M}$ PBS $(\mathrm{pH} 7.4)$, then for $2 \times 5$ minutes in $0.1 \mathrm{M}$ PBS containing $100 \mathrm{mM}$ glycine. These were then treated for 15 minutes with $0.1 \mathrm{M}$ PBS containing $0.3 \%$ Triton $^{\circledR} \mathrm{X}-100$ and subsequently washed for $2 \times 5$ minutes in 0.1 M PBS. Tissue sections were permeabilised for 20 minutes at $37^{\circ} \mathrm{C}$ with $\mathrm{TE}$ buffer (10 $\mathrm{mM}$ Tris- $\mathrm{HCl} \mathrm{pH} 8.0$ and $1 \mathrm{mM} \mathrm{Na} 2$.EDTA) containing 1

$\mu \mathrm{g} / \mathrm{mL}$ RNase-free Proteinase $\mathrm{K}$, then post-fixed for 5 minutes at $4^{\circ} \mathrm{C}$ with $0.1 \mathrm{M}$ PBS containing $4 \%$ paraformaldehyde. Sections were washed for $2 \times 5$ minutes in 0.1 M PBS then acetylated on a rocking platform by incubation for $2 \mathrm{x} 5$ minutes with $0.1 \mathrm{M}$ triethanolamine (TEA) buffer, $\mathrm{pH} 8.0$ containing $0.25 \%(\mathrm{v} / \mathrm{v})$ acetic anhydride 
(added immediately before incubation). Finally sections were incubated for 30 minutes at $37^{\circ} \mathrm{C}$ in prehybridisation buffer.

\subsubsection{In situ hybridisation}

Prehybridisation buffer was drained from the slides and each section was overlaid with $65 \mu \mathrm{L}$ of hybridisation buffer [5x SSC containing $40 \%(\mathrm{v} / \mathrm{v})$ deionised formamide, $10 \mathrm{mM}$ 1,4-Dithiothreitol Threo-1,4-dimercapto-2,3-butanediol (DTT), 1 $\mathrm{mg} / \mathrm{mL}$ denatured sheared salmon sperm DNA] and $\sim 6 \mathrm{ng}$ of labelled riboprobe. Samples were covered with Gene Frame ${ }^{\circledR}$ coverslips and incubated overnight at $40^{\circ} \mathrm{C}$ in a water bath.

\subsubsection{Posthybridisation}

Gene Frames ${ }^{\circledR}$ and coverslips were removed and the sections were washed for $2 \times 15$ minutes at $37^{\circ} \mathrm{C}$ in $2 \times \mathrm{SSC}$ and then for $2 \times 15$ minutes at $37^{\circ} \mathrm{C}$ in $1 \mathrm{x} \mathrm{SSC}$. Subsequently slides were washed for 20 minutes at $37^{\circ} \mathrm{C}$ in $2 \mathrm{x}$ SSC containing $40 \%$ (v/v) deionised formamide. Nuclei were stained by a 15 minute wash in $0.1 \mathrm{x}$ SSC containing $5 \mathrm{ng} / \mathrm{mL}$ DAPI (4',6-Diamidine-2'-phenylindole dihydrochloride), finally slides were rinsed for 5 minutes in $0.1 \mathrm{M}$ PBS. Slides were dried and $10 \mu \mathrm{L}$ of VECTASHIELD ${ }^{\circledR}$ Mounting Medium (Vector Laboratories) was added to prevent the rapid loss of fluorescence during microscopic examination; $22 \times 22 \mathrm{~mm}$, number 0 glass coverslips were placed on sections and sealed with nail varnish. 


\subsection{Analysis of FISH experiments}

\subsubsection{Fluorescent microscopy}

Slides were viewed with an Olympus AX70 photomicroscope fitted with the appropriate narrow band filters specific to DAPI, fluorescein and CY3. These filters work by shutting out the wavelengths of light that a particular fluorophore does not fluoresce under. Therefore allowing a tissue section to be labelled with more than one probe.

\subsubsection{FISH Images}

Images were captured using a Nikon Coolpix 995 digital camera. The settings used to capture the results of FISH experiments were as follows: Aperture of F5.6, maximum zoom, 1 second exposure time and unless otherwise stated all images were captured under the $20 \mathrm{x}$ objective. These images were overlayed to produce dual colour and/or mosaic images using Adobe Photoshop version 5.0 software. 


\section{Appendix A}

\section{Size standards and markers}

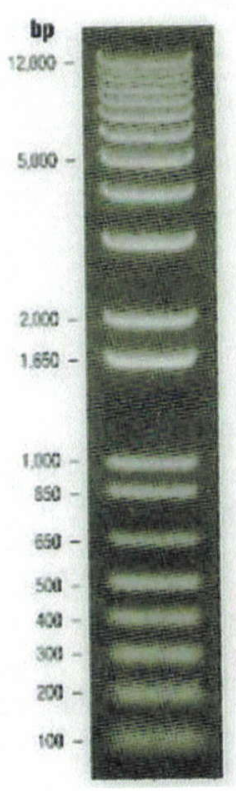

Figure A1. 1Kb+ DNA Ladder size standard (Invitrogen ${ }^{T M}$ life technologies). $900 \mathrm{ng}$ of ds DNA was loaded into this lane and run on a $0.9 \%$ agarose gel by electrophoresis. An aliquot of the $1 \mathrm{~Kb}^{+}$DNA ladder was run with all samples in this project and was used to estimate the size and amount of product present.

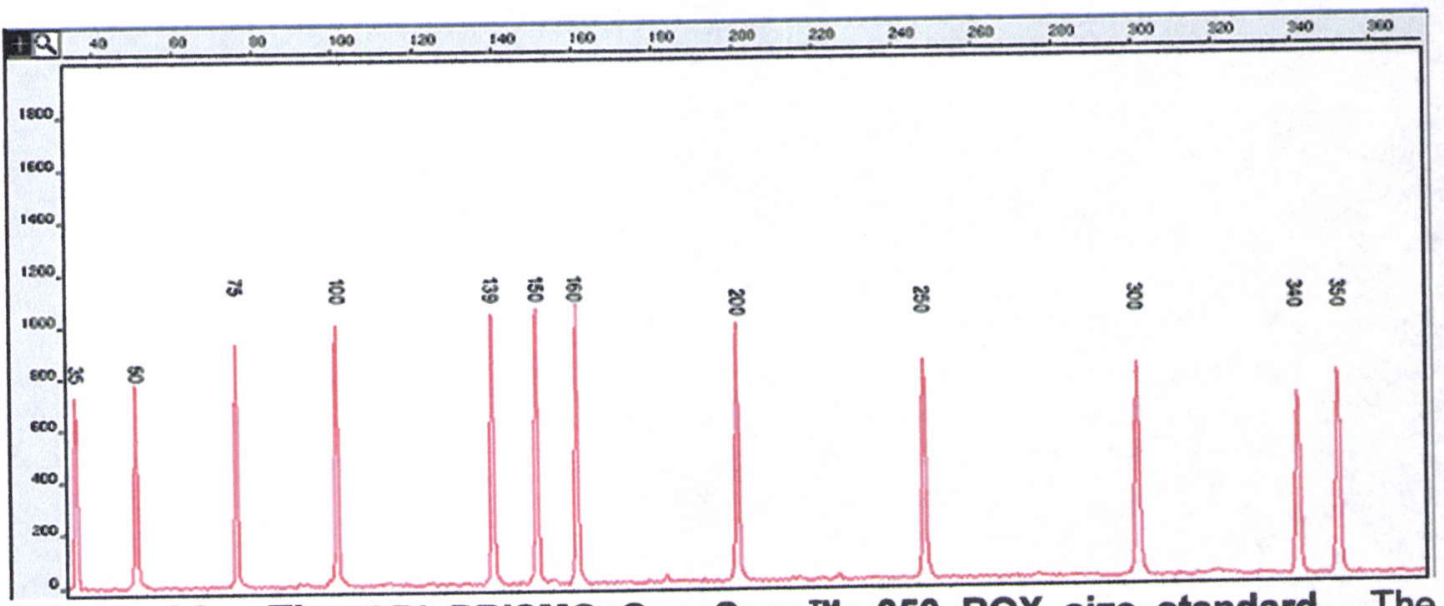

Figure A2. The ABI PRISM® GeneScan ${ }^{\mathrm{TM}}-350$ ROX size standard. The GeneScan $^{\text {TM }} 350$ ROX size standards are sets of fluorescent-labelled DNA fragments of known sizes (shown above) used for determining the size of fluorescently labelled products run on $A B I P R I S M \otimes D N A$ sequencers. 


\section{Appendix B}

\section{Chromatogram of sequenced MOR exon 3 riboprobe}

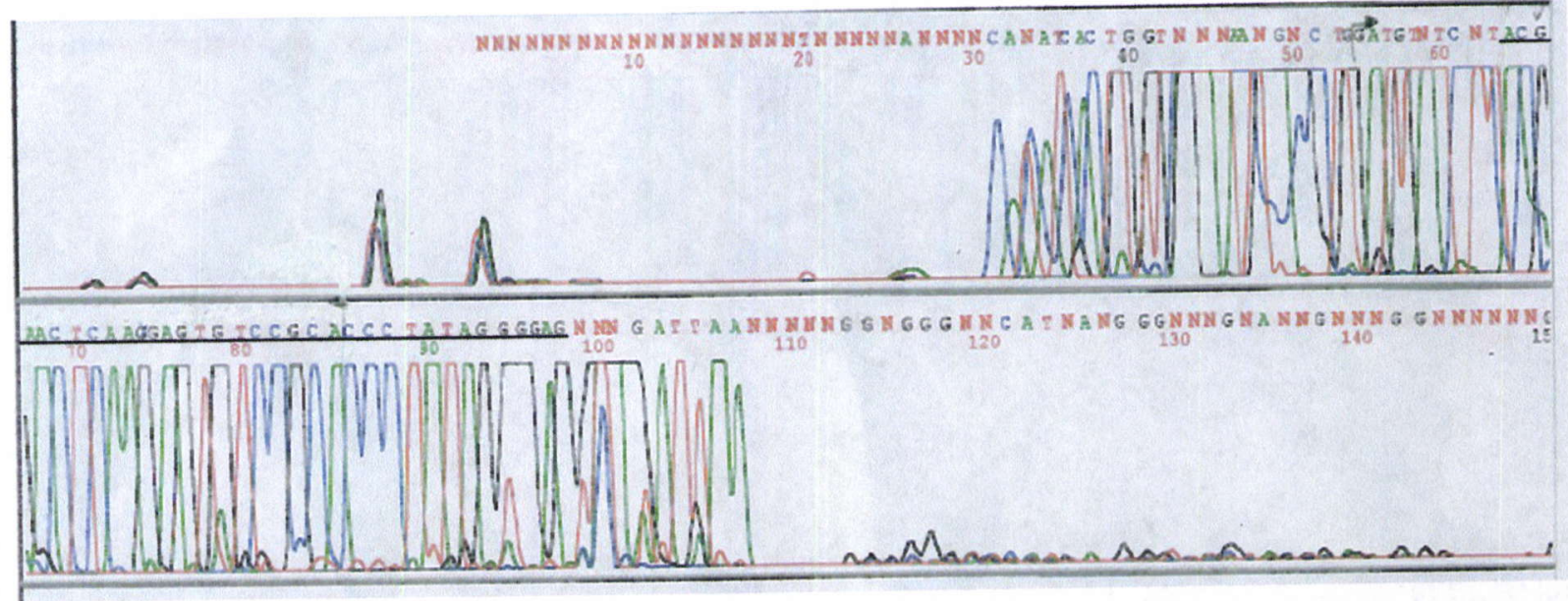

Figure B1a. Basecaller-377 version 3.7 analysis (Applied Biosystems) of ds DNA template for exon 3 riboprobe synthesis using the exon 3 forward primer for sequencing. The forward exon 3 primer was used in this sequencing reaction; approximately 70 bases were successfully called. The $\mathbf{N}$ depicts bases that were unable to be called; these bases were called manually by analysis of the chromatogram. The complimentary sequence to the MOR exon 3 T7-tailed reverse primer is underlined above.

\section{5' ACGAACTCAAGGAGTGTCCGCACCCTATAGGGAG 3' 3' TGC TGAGTT CTCACAgGCGTGgGATATCACTC 5'}

Figure B1b. Comparison of exon 3 sequence generated using the exon 3 forward primer (above) and the exon 3 reverse primer sequence. The sequence shown in red above is the underlined sequence shown in Figure B1a. The blue bases represent miscalled peaks using the Basecaller software version 3.7. The black sequence is that of the exon 3 reverse primer, it is possible to see that the reverse primer sequence has been incorporated into the template, this suggests that the ds exon 3 template has been synthesised correctly. 


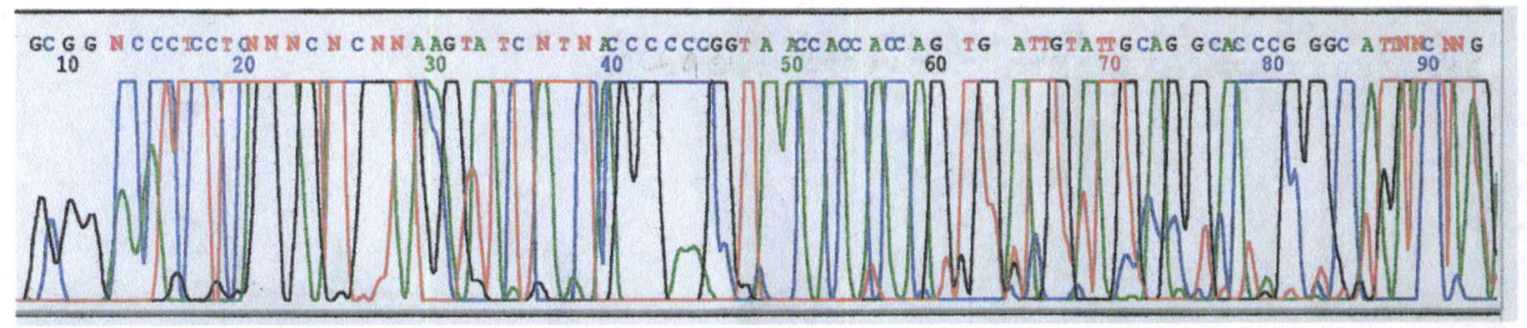

Figure B2. Basecaller-377 version 3.7 analysis (Applied Biosystems) of ds DNA template for exon 3 riboprobe synthesis. The reverse exon 3 primer was used in this sequencing reaction; approximately 34 bases were successfully called. The $\mathrm{N}$ depicts bases that were unable to be called; these bases were called manually by analysis of the chromatogram. The complimentary sequence to the MOR exon 3 forward primer can be identified above.

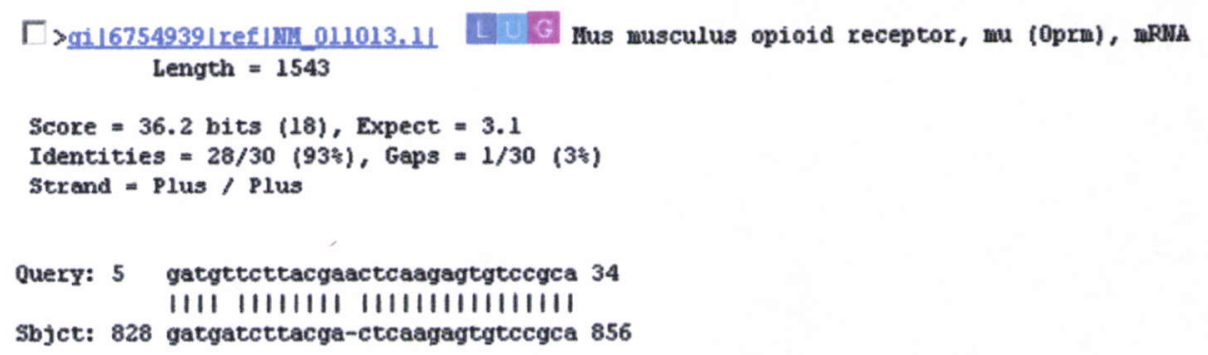

Figure B3 First BLAST alignment for the sequence of the ds DNA template used in exon 3 riboprobe synthesis. These results show a $93 \%$ identity of the sequence exon 3 riboprobe template to MOR mRNA.

GCC T CCCAAC AAA CCC T G ACA G C AA C C T G ATT C C AAG T A G A T G G C A G C C T C

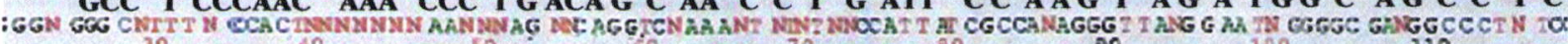

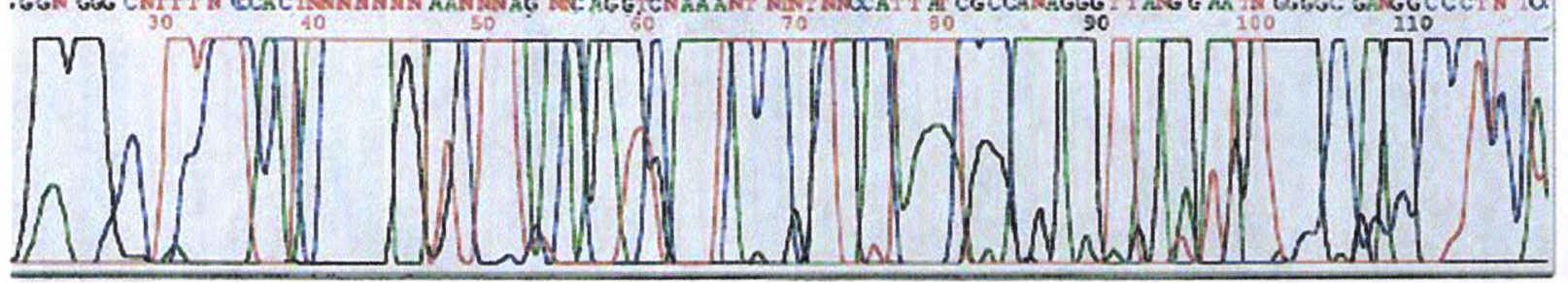

Figure B4 Basecaller-377 version 3.7 analysis (Applied Biosystems) of ds DNA template for exon 4 riboprobe synthesis. The reverse exon 4 primer was used in this sequencing reaction; approximately 51 bases were successfully called. The $\mathrm{N}$ depicts bases that were unable to be called; these bases were called manually by analysis of the chromatogram. The actual sequence to the MOR exon 4 forward primer can be identified above in bold type.

NB. Refer Figure 3.11 for the top two BLAST search results with the sequence base called for exon 4, shown above Figure B4. 


\title{
Appendix C
}

\section{Design of MOR primers for riboprobe synthesis}

\begin{abstract}
1 tccttccagg gtccatagat tgcaccctca cgttctctca tcccacatgg tactgggaga
61 acctgctcaa aatctgtgtc ttcatcttcg ccttcatcat gccggtcctc atcatcactg

121 tgtgttatgg actgatgatc ttacgactca agagtgtccg catgctatcg ggctccaaag

181 aaaaggacag gaacctgcgc aggatcaccc ggatggtgct ggtggtcgtg gctgtattta

241 ttgtctgctg gacccccatc cacatctatg tcatcatcaa agcactgatc acgattccag

301 aaaccacttt ccagactgtt tcctggcact tctgcattgc cttgggttac acaaacagct
\end{abstract}

Figure C1 Exon 3 riboprobe designed to MOR-1 mRNA. The nucleotides shown in bold represent the forward and T7-tailed reverse primer sequences. The exon 3 riboprobe was designed to the Mus musculus mu opioid receptor (MOR) gene, accession number: U10560.

$1 \mathrm{ttcttcagc} \mathrm{tagaaaatct} \mathrm{ggaagcagaa} \mathrm{actgctccat} \mathrm{tgccctaact} \mathrm{gggtcccacg}$

61 ccatccagac cctcgctaaa cttagaggct gccatctact tggaatcagg ttgctgtcag

121 ggttgtggg aggcgctggt ttcctggaaa agcatctgat cctgc

Figure C2 Overlapping oligonucleotides designed to exon 4 of MOR-1 mRNA. The area underlined in red represents the region that the forward and T7-tailed oligonucleotides (represented in bold type) overlap. The overlapping oligos were designed to the Mus musculus MOR-1 gene, accession number: U10561.

1 atcagacgct cagacgttcc cttctgcctg ccgctcttct ctggttccac tagggctggt

61 ccatgtaaga atctgacgga gcctagggca gctgtgagag gaagaggctg gggcgcgtgg

121 aacccgaaaa gtctgagtgc tctcagttac agcctaccta gtccgcagca ggcttcagc

181 accatggaca gcagcaccgg cccagggaac accagcgact gctcagaccc cttagctcag

241 gcaagttgct ccccagcacc tggctcctgg ctcaacttgt cccacgttga tggcaaccag

301 tccgatccat gcggtctgaa ccgcaccggg cttggcggga acgacagcct gtgccctcag

361 accggcagcc cttccatggt cacagccatt accatcatgg ccctctactc tatcgtgtgt

Figure C3a MOR 287 fragment designed to MOR-1 mRNA. The forward and T7-tailed reverse primers are shown in bold type. The forward primer begins in the 5' UTR of exon 1 and the reverse primer begins at the end of exon 1 (Yoshikawa et al. 1999). The accession number for the MOR-1 mRNA is NM_013071. 
Forward primer sequence: 5' CCT GCC GCT CTT CTC GGT C 3'

Reverse primer sequence: 5' TAA TAC GAC TCA CTA TAG GGC GCA TGG AT C GGA

CTG GTT G 3'

Figure C3b Primer sequences used to generate the MOR 287 fragment Forward primer was designed by Yoshikawa et al. 1999 and the reverse T7-tailed primer (shown in green print) was designed by Eli Mrkusich, 2001.

1 gggaacaccc ctccacggct aatacagtgg atcgaactaa ccaccagagg aatgaggaac

61 cttcttcctg atgatggccc aagacaggaa tccggggaag gccagcttgg caggtgaatg

121 tcatccgaac acagggatga gctggtgagc agtgtggagg tgcaggctct gctagaagac 181 agcgagaggg aagatgcctt tcactcataa aggttaggag ttgtaagtgt aatgtctaat

241 gtattgtctg gtttgccgta ttgcgtgttt tcatacgaat gacttggcaa tgtagagaca

301 gcctgttaaa catggcaatg tggcttctgt ggacaccgct ctcatgccac tgtgtgcaaa

361 taaacaaggg acttccttag gagcagccta gccaccttac atgaagagtt tcctaatgtc

421 acagaagagg aacgaaagcc agtattgtt atgacactgt ttcttctaaa taatgtaatt

481 aataaaataa agaaaaacat ggacctcgaa aaaaaaaaaa aaaaaaaaaa aaaaa

Figure C4 The 3'RACE sequence of MOR-1C mRNA used to design the exon 9 overlapping oligonucleotides. The sequence shown in bold type represents the entire sequence used to generated 4 overlapping oligonucleotides that overlapped by 18 nts. The last oligonucleotide (called D) was T7-tailed, shown in green above. The accession number for this sequence is: AF062752.

901 ccgaaatgcc aaaattgtca atgtctgcaa ctgg atcctc tcttctgcca ttggtctgcc 961 cgtaatgttc atggcaacca caaaatacag gcaggggtcc atagattgca ccctcacgtt

1021 ctctcatccc acatggtact gggagaacct gctcaaaatc tgtgtcttca tcttcgcctt

1081 catcatgccg gtcctcatca tcactgtgtg ttatggactg atgatcttac gactcaagag

1141 tgtccgcatg ctgtcgggct ccaaagaaaa ggacaggaac ctgcgcagga tcacccggat

Figure C5 Target region of CLiP designed to Mus musculus MOR-1 mRNA. The text shown in bold is the MOR-1 target sequence for the CLiPs designed and utilised in this project. The blue vertical line represents the ligation junction. The accession number for the Mus musculus MOR-1 mRNA is AF400248. 


\section{Appendix D}

\section{Staining of different structures in adult rat brain using}

riboprobes

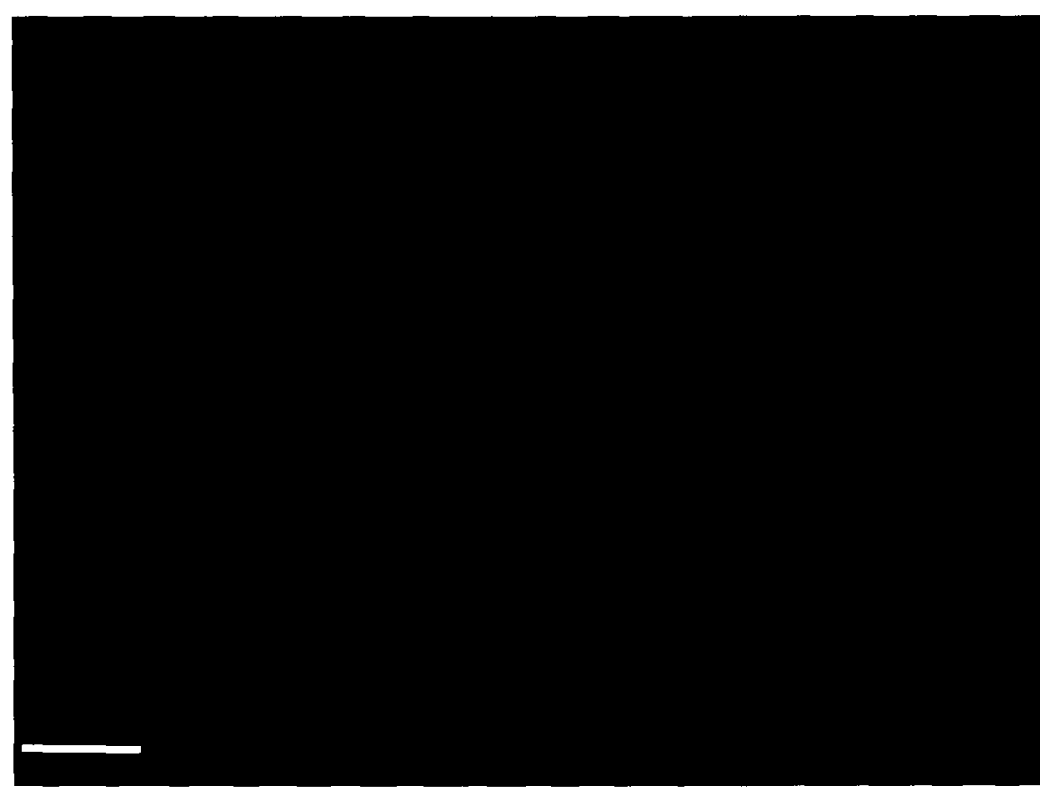

Figure D1. FITC-labelled exon 4 riboprobes staining the dendate gyrus (DG). Fluorescent photomicrograph showing exon 4 riboprobes localising to the dense granular layer of the DG and to single neurons inside the DG. Scale bar $=200 \mu \mathrm{m}$.

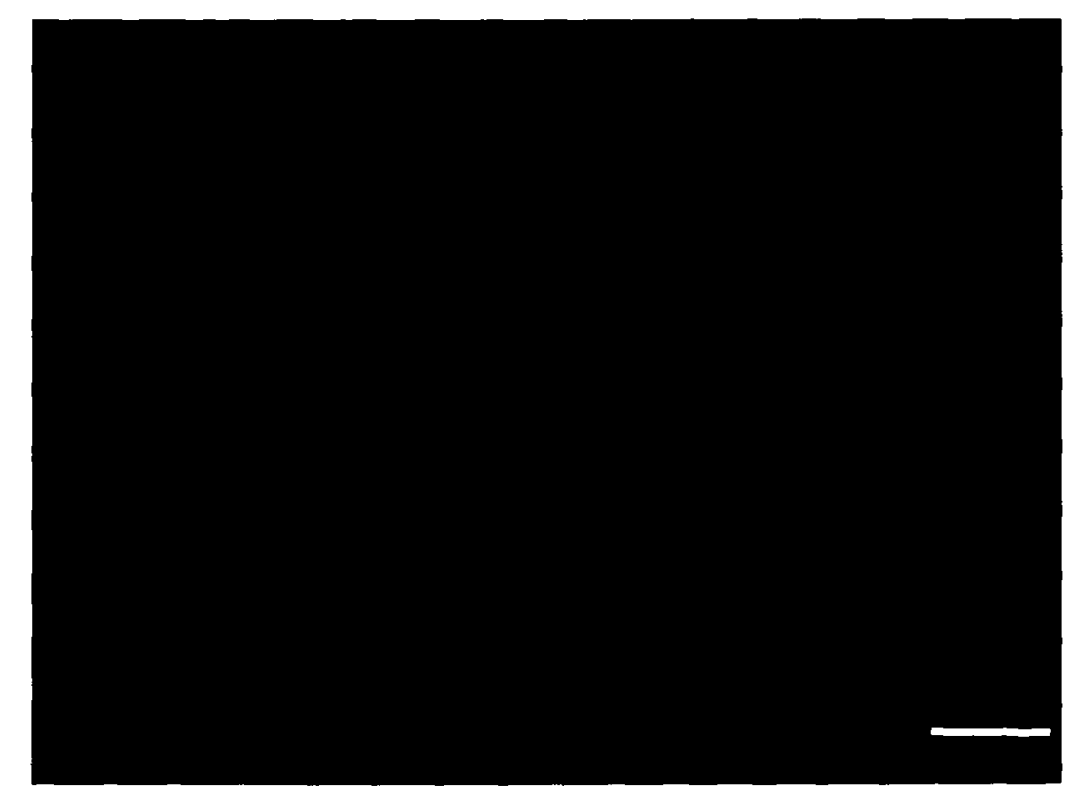

Figure D2. FITC-labelled Pvu II generated riboprobes. Fluorescent photomicrographs of a sagittal section through the dorsal endopiriform nucleus in adult rat brain. This riboprobe generated to the 5' UTR and exon 1 of the MOR, appears to have localised to the cytoplasm of the dorsal endopiriform nuclei. Scale bar $=200 \mu \mathrm{m}$. 


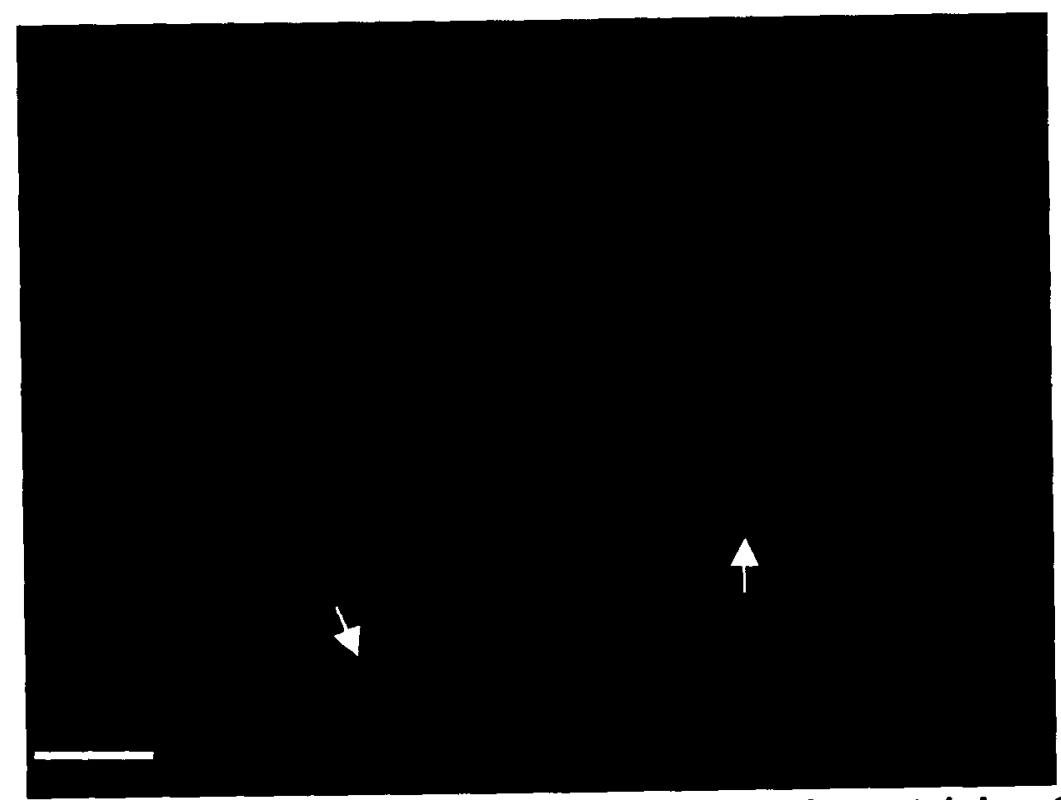

Figure D3. FITC-labelled Pvu II generated riboprobes staining the anterior and posterior interposed cerebellar nuclei. These riboprobes appear to be localised to individual neurons and some dendritic staining appears to be present (arrows). Scale bar $=200 \mu \mathrm{m}$.

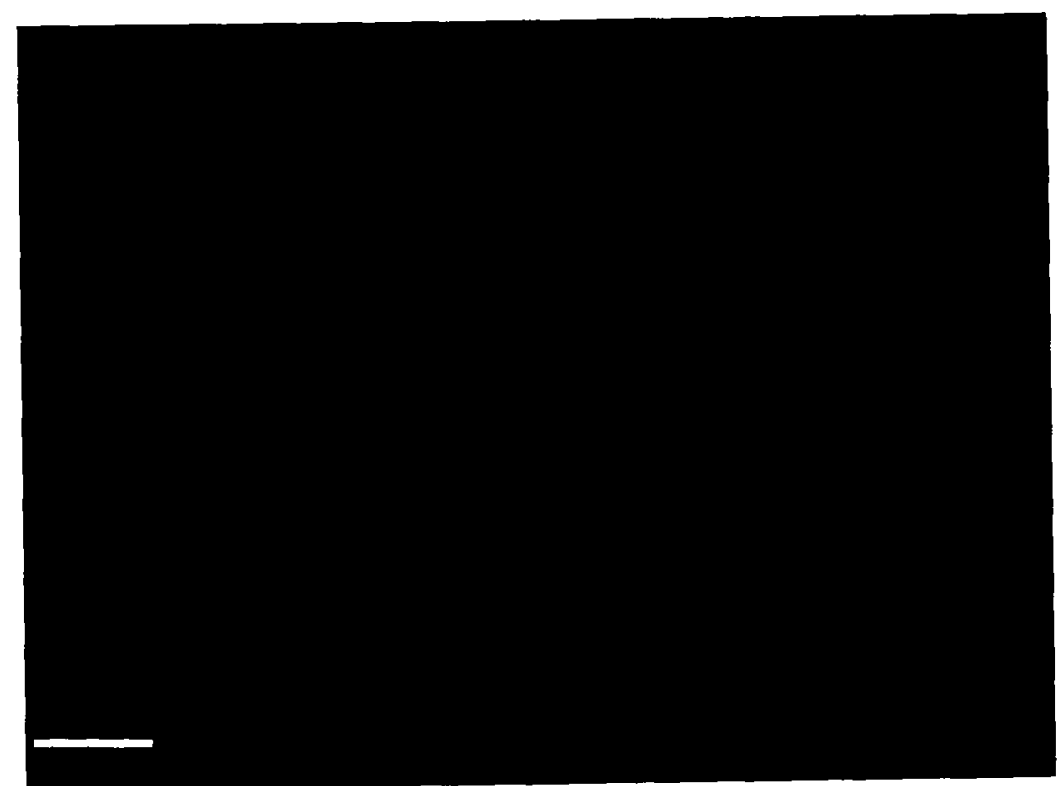

Figure D4. Exon 9 riboprobes staining in the parvocellular lateral reticular nucleus. The appearance of the staining above is typical of non-specific binding, as no clear cytoplasmic staining is visible. Scale bar $=200 \mu \mathrm{m}$. 
Appendix E

\section{$\underline{\text { Raw data of signal and background intensities }}$}

\begin{tabular}{|c|c|c|c|c|c|c|}
\hline & Figure & Riboprobe & Mean & $\begin{array}{r}\text { Std } \\
\text { dev. }\end{array}$ & Median & $\begin{array}{r}\text { Difference (signal to } \\
\text { background using } \\
\text { median value) }\end{array}$ \\
\hline Signal & 3.14 & CY3-Ex3 & 103.08 & 8.55 & 103 & \\
\hline Background & 3.14 & CY3-Ex3 & 81.47 & 2.81 & 82 & 21 \\
\hline Signal & $3.15, \mathrm{~A}$ & FITC-Ex4 & 77.42 & 2.60 & 78 & \\
\hline $\begin{array}{l}\text { Background } \\
\text { GL }\end{array}$ & $3.15, \mathrm{~A}$ & FITC-Ex4 & $\begin{array}{l}35.30 \\
58.77 \\
\end{array}$ & $\begin{array}{l}2.30 \\
3.44 \\
\end{array}$ & $\begin{array}{l}35 \\
59 \\
\end{array}$ & $\begin{array}{l}43 \\
19 \\
\end{array}$ \\
\hline Signal & $3.15, \mathrm{C}$ & FITC-Ex4 & 68.81 & 5.66 & 70 & \\
\hline Background & $3.15, \mathrm{C}$ & FITC-Ex4 & 41.13 & 2.49 & 41 & 29 \\
\hline Signal & $3.15, \mathrm{E}$ & FITC-Ex4 & 92.71 & 7.95 & 94 & \\
\hline Background & $3.15, \mathrm{E}$ & FITC-Ex4 & 42.77 & 2.08 & 43 & 51 \\
\hline Signal & $3.16, \mathrm{~A}$ & FITC-Ex 9 & 78.18 & 4.41 & 78 & \\
\hline Background & $3.16, \mathrm{~A}$ & FITC-Ex9 & \begin{tabular}{|l|}
57.31 \\
\end{tabular} & 1.67 & 57 & 21 \\
\hline Signal & $3.16, \mathrm{C}$ & CY3-Ex9 & 99.09 & 12.89 & 99 & \\
\hline Background & $3.16, \mathrm{C}$ & CY3-Ex9 & 60.05 & 4.03 & 60 & 39 \\
\hline Signal & $3.17, \mathrm{~A}$ & FITC-Ex 1 & 83.62 & 7.85 & 82 & \\
\hline $\begin{array}{l}\text { Background } \\
\text { GL }\end{array}$ & $3.17, \mathrm{~A}$ & FITC-Ex 1 & \begin{tabular}{|l|}
38.95 \\
72.06 \\
\end{tabular} & \begin{tabular}{|l|}
1.68 \\
2.45 \\
\end{tabular} & $\begin{array}{l}39 \\
72 \\
\end{array}$ & $\begin{array}{l}43 \\
10 \\
\end{array}$ \\
\hline Signal & $3.17, \mathrm{D}$ & FITC-Ex 1 & 63.19 & 5.65 & 63 & \\
\hline Background & $3.17, \mathrm{D}$ & FITC-Ex1 & 32.98 & 2.40 & 33 & 30 \\
\hline Signal & $3.18, \mathrm{~A}$ & FITCPvu2 & 71.50 & 6.04 & 71 & \\
\hline Background & $3.18, \mathrm{~A}$ & FITCPvu2 & 17.38 & 1.83 & 17 & 54 \\
\hline Signal & $3.18, \mathrm{C}$ & FITCPvu2 & 79.57 & $15.7 \overline{3}$ & 82 & \\
\hline Background & $3.18, \mathrm{C}$ & FITCPvu2 & 18.95 & 2.39 & 19 & 63 \\
\hline Signal & $3.18, \mathrm{E}$ & FITCPvu2 & 86.12 & 6.13 & 87 & \\
\hline $\begin{array}{l}\text { Background } \\
\text { GL }\end{array}$ & $3.18, \mathrm{E}$ & FITCPvu2 & \begin{tabular}{|l|}
28.57 \\
50.01 \\
\end{tabular} & \begin{tabular}{|l|}
1.87 \\
4.05 \\
\end{tabular} & $\begin{array}{l}29 \\
50 \\
\end{array}$ & \begin{tabular}{|l|}
58 \\
37 \\
\end{tabular} \\
\hline Signal & $3.19 \mathrm{a}, \mathrm{A}$ & FITC-Ex 1 & 104.37 & 7.29 & 106 & \\
\hline $\begin{array}{l}\text { Background } \\
\text { GL }\end{array}$ & $3.19 \mathrm{a}, \mathrm{A}$ & FITC-Ex I & \begin{tabular}{|l|}
45.96 \\
82.51 \\
\end{tabular} & \begin{tabular}{|l|}
2.73 \\
4.16 \\
\end{tabular} & $\begin{array}{l}46 \\
83 \\
\end{array}$ & $\begin{array}{l}60 \\
23 \\
\end{array}$ \\
\hline Signal & $3.19 \mathrm{a}, \mathrm{B}$ & CY3-Ex4 & 120.04 & 9.98 & 124 & \\
\hline $\begin{array}{l}\text { Background } \\
\text { GL }\end{array}$ & $3.19 \mathrm{a}, \mathrm{B}$ & CY3-Ex4 & $\begin{array}{l}79.53 \\
99.02 \\
\end{array}$ & $\begin{array}{l}3.53 \\
7.90 \\
\end{array}$ & $\begin{array}{l}79 \\
99 \\
\end{array}$ & \begin{tabular}{|l|}
45 \\
25 \\
\end{tabular} \\
\hline Signal & $3.19 a, D$ & FITCPvu2 & 118.58 & 12.06 & 120 & \\
\hline $\begin{array}{l}\text { Background } \\
\text { GL }\end{array}$ & $3.19 \mathrm{a}, \mathrm{D}$ & FITCPvu2 & \begin{tabular}{|l|}
38.23 \\
72.74 \\
\end{tabular} & \begin{tabular}{|l|}
2.82 \\
3.52 \\
\end{tabular} & \begin{tabular}{|l|}
38 \\
73 \\
\end{tabular} & \begin{tabular}{|l|}
82 \\
47 \\
\end{tabular} \\
\hline Signal & $3.19 \mathrm{a}, \mathrm{E}$ & CY3-Ex4 & 99.03 & 4.75 & 100 & \\
\hline $\begin{array}{l}\text { Background } \\
\text { GL }\end{array}$ & $3.19 \mathrm{a}, \mathrm{E}$ & CY3-Ex4 & \begin{tabular}{|l|}
70.34 \\
86.83 \\
\end{tabular} & \begin{tabular}{|l|}
2.66 \\
4.35 \\
\end{tabular} & $\begin{array}{l}70 \\
86 \\
\end{array}$ & \begin{tabular}{|l|}
30 \\
14 \\
\end{tabular} \\
\hline Signal & $3.19 \mathrm{~b}, \mathrm{~A}$ & FITC-287 & 81.74 & 3.96 & 81 & \\
\hline $\begin{array}{l}\text { Background } \\
\text { GL }\end{array}$ & $3.19 \mathrm{~b}, \mathrm{~A}$ & FITC-287 & \begin{tabular}{|l|}
34.14 \\
58.45 \\
\end{tabular} & \begin{tabular}{|l|}
1.66 \\
3.12 \\
\end{tabular} & $\begin{array}{l}34 \\
58 \\
\end{array}$ & $\begin{array}{l}47 \\
23 \\
\end{array}$ \\
\hline Signal & $3.19 \mathrm{~b}, \mathrm{~B}$ & CY3-Ex4 & 116.89 & 5.56 & 118 & \\
\hline $\begin{array}{l}\text { Background } \\
\text { GL }\end{array}$ & $3.19 \mathrm{~b}, \mathrm{~B}$ & CY3-Ex4 & \begin{tabular}{|l|}
81.25 \\
106.46 \\
\end{tabular} & \begin{tabular}{|l|}
1.99 \\
3.33 \\
\end{tabular} & $\begin{array}{l}81 \\
106 \\
\end{array}$ & \begin{tabular}{|l|}
37 \\
12 \\
\end{tabular} \\
\hline Signal & $3.19 \mathrm{~b}, \mathrm{D}$ & FITC-287 & 74.66 & 4.94 & 75 & \\
\hline $\begin{array}{l}\text { Background } \\
\text { GL }\end{array}$ & $3.19 \mathrm{~b}, \mathrm{D}$ & FITC-287 & \begin{tabular}{|l|}
53.36 \\
34.05 \\
\end{tabular} & $\begin{array}{l}3.58 \\
2.15\end{array}$ & \begin{tabular}{|l|}
54 \\
34
\end{tabular} & $\begin{array}{l}41 \\
21 \\
\end{array}$ \\
\hline
\end{tabular}




\begin{tabular}{|l|l|l|l|l|l|l|}
\hline & Figure & Riboprobe & Mean & $\begin{array}{r}\text { Std } \\
\text { dev. }\end{array}$ & Median & $\begin{array}{l}\text { Difference (signal to } \\
\text { background using } \\
\text { median value) }\end{array}$ \\
\hline Signal & $3.19 \mathrm{~b}, \mathrm{E}$ & CY3-Ex4 & 126.16 & 4.51 & 125 & \\
\hline $\begin{array}{l}\text { Background } \\
\text { GL }\end{array}$ & $3.19 \mathrm{~b}, \mathrm{E}$ & CY3-Ex4 & $\begin{array}{l}86.09 \\
99.64\end{array}$ & $\begin{array}{l}3.10 \\
2.79\end{array}$ & $\begin{array}{l}86 \\
100\end{array}$ & 39 \\
25
\end{tabular}

Figure E1. Pixel intensity data obtained from histogram analysis using Adobe Photoshop version 5.5. Unmodified images were enlarged and one stained neuron was analysed for its intensity, the background was then analysed for intensity using the identical size area as the neuron and the same filter set. $\mathbf{G L}=$ represents the densely packed neurons of the granular layer. NB. The background intensity for the cerebellum was taken in the molecular layer.

\section{Example calculation of \% signal is greater than background}

Figure 3.14 gives a signal of 103 pixels for the CY3-labelled exon 3 riboprobe, the median value for the background was determined to be 82 pixels. This means the signal is 21 pixels greater than the background. To express this as a percentage, the difference of 21 is divided by the signal and multiplied by 100, e.g.

$21 / 103 \times 100=20.39 \%$

This value implies that the signal seen for the exon 3 riboprobe is only $20 \%$ higher than the background and, as this is a low value, not much confidence was placed in this result. 


\section{References}

Abbadie, C., Y. X. Pan, and G. W. Pasternak. 2000a. Differential distribution in rat brain of mu opioid receptor carboxy terminal splice variants MOR-1C-like and MOR-1-like immunoreactivity: evidence for region-specific processing. $\mathrm{J}$ Comp Neurol 419: 244-56.

Abbadie, C., G. W. Pasternak, and S. A. Aicher. 2001. Presynaptic localization of the carboxy-terminus epitopes of the mu opioid receptor splice variants MOR-1C and MOR-1D in the superficial laminae of the rat spinal cord. Neuroscience 106: 833-42.

Abbadie, C., Y. Pan, C. T. Drake, and G. W. Pasternak. 2000b. Comparative immunohistochemical distributions of carboxy terminus epitopes from the mu-opioid receptor splice variants MOR-1D, MOR-1 and MOR-1C in the mouse and rat CNS. Neuroscience 100: 141-53.

Abeyta, A., T. S. Dettmer, A. Barnes, D. Vega, M. Carta, N. Gallegos, M. Raymond-Stintz, D. D. Savage, C. F. Valenzuela, and L. C. Saland. 2002. Delta opioid receptor localization in the rat cerebellum. Brain Res 931: 100-5.

Ableitner, A. 1994. Brain sites involved in delta-opioid receptor-mediated actions. Eur J Pharmacol 271: 213-22.

Abramowitz, M., K. I. Tchourioukanov, and M. W. Davidson. 2003. Fluorescence Combination Microscopy.

Alvarez, V. A., S. Arttamangkul, V. Dang, A. Salem, J. L. Whistler, M. Von Zastrow, D. K. Grandy, and J. T. Williams. 2002. mu-Opioid receptors: Ligand-dependent activation of potassium conductance, desensitization, and internalization. J Neurosci 22: 5769-76.

Antson, D. O., A. Isaksson, U. Landegren, and M. Nilsson. 2000. PCR-generated padlock probes detect single nucleotide variation in genomic DNA. Nucleic Acids Res 28: E58.

Attali, B., and Z. Vogel. 1990. Characterization of kappa opiate receptors in rat spinal cord-dorsal root ganglion cocultures and their regulation by chronic opiate treatment. Brain Res 517: 182-8.

Atweh, S. F., and M. J. Kuhar. 1983. Distribution and physiological significance of opioid receptors in the brain. Br Med Bull 39: 47-52.

Baner, J., M. Nilsson, M. Mendel-Hartvig, and U. Landegren. 1998. Signal amplification of padlock probes by rolling circle replication. Nucleic Acids Res 26: 5073-8.

Barany, F. 1991. Genetic disease detection and DNA amplification using cloned thermostable ligase. Proc Natl Acad Sci U S A 88: 189-93.

Bare, L. A., E. Mansson, and D. Yang. 1994. Expression of two variants of the human mu opioid receptor mRNA in SK-N-SH cells and human brain. FEBS Lett 354: 213-6.

Barg, J., and R. Simantov. 1989. Developmental profile of kappa, mu and delta opioid receptors in the rat and guinea pig cerebellum. Dev Neurosci 11:42834.

Bayon, A., W. J. Shoemaker, F. E. Bloom, A. Mauss, and R. Guillemin. 1979. Perinatal development of the endorphin- and enkephalin-containing systems in the rat brain. Brain Res 179: 93-101. 
Borgland, S. L. 2001. Acute opioid receptor desensitization and tolerance: is there a link? Clin Exp Pharmacol Physiol 28: 147-54.

Brown, G. P., K. Yang, O. Ouerfelli, K. M. Standifer, D. Byrd, and G. W. Pasternak. 1997. 3H-morphine-6beta-glucuronide binding in brain membranes and an MOR-1-transfected cell line. J Pharmacol Exp Ther 282: 1291-7.

Brownstein, M. 1993. A Brief History of Opiates, Opioid Peptides, and Opioid Receptors. PNAS 90: 5391-5393.

Buono, R. J., and R. K. Lang. 1999. Hypoxic repression of lactate dehydrogenase$\mathrm{B}$ in retina. Exp Eye Res 69: 685-93.

Butour, J. L., C. Moisand, H. Mazarguil, C. Mollereau, and J. C. Meunier. 1997. Recognition and activation of the opioid receptor-like ORL 1 receptor by nociceptin, nociceptin analogs and opioids. Eur J Pharmacol 321: 97-103.

Chaiet, L., and E. J. Wolf. 1964. The properties of streptavidin, a biotin-binding protein produced by Streptomyces. Arch. Biochem. Biophys 108: 1-5.

Chen, Y., A. Mestek, J. Liu, J. A. Hurley, and L. Yu. 1993. Molecular cloning and functional expression of a mu-opioid receptor from rat brain. Mol Pharmacol 44: 8-12.

Cheng, J., B. P. Roques, G. A. Gacel, E. Huang, and G. W. Pasternak. 1992. Kappa 3 opiate receptor binding in the mouse and rat. Eur J Pharmacol 226: 15-20.

Cheung, S. W., P. V. Tishler, L. Atkins, S. K. Sengupta, E. J. Modest, and B. G. Forget. 1977. Gene mapping by fluorescent in situ hybridization. Cell Biol Int Rep 1: 255-62.

Commons, K. G., E. J. Van Bockstaele, and D. W. Pfaff. 1999. Frequent colocalization of mu opioid and NMDA-type glutamate receptors at postsynaptic sites in periaqueductal gray neurons. The Journal of Comparative Neurology 408: 549-559.

Corbett, A., S. McKnight, and G. Henderson. Opioid Receptors. http://www.opioids.com/receptors.

Cox, B. M., and M. Weinstock. 1964. Quantitative studies of the antagonism by nalorphine of some of the actions of morphine-like analgesic drugs. Br J Pharmacol 22: 289-300.

Cvejic, S., and L. A. Devi. 1997. Dimerization of the delta opioid receptor: implication for a role in receptor internalization. J Biol Chem 272: 26959-64.

Day, D. J., P. W. Speiser, P. C. White, and F. Barany. 1995. Detection of steroid 21-hydroxylase alleles using gene-specific PCR and a multiplexed ligation detection reaction. Genomics 29: 152-62.

Delhanty, J. D., J. C. Harper, A. Ao, A. H. Handyside, and R. M. Winston. 1997. Multicolour FISH detects frequent chromosomal mosaicism and chaotic division in normal preimplantation embryos from fertile patients. Hum Genet 99: 755-60.

Dieffenbach, C. W., T. M. J. Lowe, and G. S. Dveksler. 1995. General Concepts for PCR Primer Design, in PCR Primer, pp. 133-155. Cold Spring Harbour Laboratory Press, New York.

Ding, Y. Q., T. Kaneko, S. Nomura, and N. Mizuno. 1996. Immunohistochemical localization of mu-opioid receptors in the central nervous system of the rat. $\mathbf{J}$ Comp Neurol 367: 375-402. 
Eisinger, D. A., H. Ammer, and R. Schulz. 2002. Chronic morphine treatment inhibits opioid receptor desensitization and internalization. J Neurosci 22: 10192-200.

Erdtmann-Vourliotis, M., P. Mayer, U. Riechert, M. Handel, J. Kriebitzsch, and V. Hollt. 1999. Rational design of oligonucleotide probes to avoid optimization steps in in situ hybridization. Brain Res Brain Res Protoc 4: 8291.

Ertsey, R., and L. M. Scavo. 1998. Coverslip mounted-immersion cycled in situ RT-PCR for the localization of mRNA in tissue sections. Biotechniques 24: 92, 94, 96, 98-100.

Evans, C. J., D. E. Keith, Jr., H. Morrison, K. Magendzo, and R. H. Edwards. 1992. Cloning of a delta opioid receptor by functional expression. Science 258: $1952-5$.

Fan, X. L., J. S. Zhang, X. Q. Zhang, W. Yue, and L. Ma. 2003. Differential regulation of beta-arrestin 1 and beta-arrestin 2 gene expression in rat brain by morphine. Neuroscience 117: 383-9.

Feldman, R. S., J. S. Meyer, and L. F. Quenzer. 1997. Principles of Neuropsychopharmacology, pp. 31-35. Sunderland, MA: Sinauer Associates, Inc.

Ferguson, S. S. 2001. Evolving concepts in G protein-coupled receptor endocytosis: the role in receptor desensitization and signaling. Pharmacol Rev 53: 1-24.

Foran, S. E., D. B. Carr, A. W. Lipkowski, I. Maszczynska, J. E. Marchand, A. Misicka, M. Beinborn, A. S. Kopin, and R. M. Kream. 2000. A substance P-opioid chimeric peptide as a unique nontolerance-forming analgesic. Proc Natl Acad Sci U S A 97: 7621-6.

Forlano, P. M., K. P. Maruska, S. A. Sower, J. A. King, and T. C. Tricas. 2000. Differential Distribution of Gonadotrophin-Releasing-HormoneImmunoreactive Neurons in the Stringray Brain: Functional and Evolutionary Considerations. General and Comparative Endocrinology 188: 226-248.

Frengen, E., P. D. Thomsen, G. Brede, J. Solheim, P. J. de Jong, and H. Prydz. 1997. The gene cluster containing the LCAT gene is conserved between human and pig. Cytogenet Cell Genet 76: 53-7.

Gaillard, C., and F. Strauss. 1990. Ethanol precipitation of DNA with linear polyacrylamide as carrier. Nucleic Acids Research 18: 378.

Gall, J. G., and M. L. Pardue. 1969. Formation and detection of RNA-DNA hybrid molecules in cytological preparations. Proc Natl Acad Sci U S A 63: 378-83.

Gastard, M. 2000. Delta-opioid receptor endocytosis in spinal cord after dermenkephalin activation. BMC Neurosci 1: 1.

Gaveriaux, C., J. Peluso, F. Simonin, J. Laforet, and B. Kieffer. 1995. Identification of kappa- and delta-opioid receptor transcripts in immune cells. FEBS Lett 369: 272-6.

Gaveriaux-Ruff, C., and B. Kieffer. 2002. Opioid receptor genes inactivated in mice: the highlights. Neuropeptides 36: 62.

Gaveriaux-Ruff, C., J. Peluso, K. Befort, F. Simonin, C. Zilliox, and B. L. Kieffer. 1997. Detection of opioid receptor mRNA by RT-PCR reveals alternative splicing for the delta- and kappa-opioid receptors. Brain Res Mol Brain Res 48: 298-304.

Georges, F., E. Normand, B. Bloch, and C. Le Moine. 1998. Opioid receptor gene expression in the rat brain during ontogeny, with special reference to the 
mesostriatal system: an in situ hybridization study. Brain Res Dev Brain Res 109: 187-99.

Go, V. L., and T. L. Yaksh. 1987. Release of substance P from the cat spinal cord. J Physiol 391: 141-67.

Goldstein, A., L. I. Lowney, and B. K. Pal. 1971. Stereospecific and nonspecific interactions of the morphine congener levorphanol in subcellular fractions of mouse brain. Proc Natl Acad Sci U S A 68: 1742-7.

Goldstein, A., W. Fischli, L. I. Lowney, M. Hunkapiller, and L. Hood. 1981. Porcine pituitary dynorphin: complete amino acid sequence of the biologically active heptadecapeptide. Proc Natl Acad Sci U S A 78: 7219-23.

Gonzalez-Guerrero, P. R., R. Rigual, and C. Gonzalez. 1993. Opioid peptides in the rabbit carotid body: identification and evidence for co-utilization and interactions with dopamine. J Neurochem 60: 1762-8.

Gozzetti, A., and M. M. Le Beau. 2000. Fluorescence in situ hybridization: uses and limitations. Semin Hematol 37: 320-33.

Gray, P. A., J. C. Rekling, C. M. Bocchiaro, and J. L. Feldman. 1999. Modulation of respiratory frequency by peptidergic input to rhythmogenic neurons in the preBotzinger complex. Science 286: 1566-8.

Gregory, J. [ed.] 1996. Handbook of Fluorescent Probes and Research Products.

Guiramand, J., J. P. Montmayeur, J. Ceraline, M. Bhatia, and E. Borrelli. 1995. Alternative splicing of the dopamine D2 receptor directs specificity of coupling to G-proteins. J Biol Chem 270: 7354-8.

Gyllensten, U. B., and H. A. Erlich. 1988. Generation of single-stranded DNA by the polymerase chain reaction and its application to direct sequencing of the HLA-DQA locus. Proc Natl Acad Sci U S A 85: 7652-6.

Hendelman, W. 1994. Student's Atlas of Neuroanatomy. W.B. Saunders Company: A Division of Harcourt Brace and Company, Philidelphia.

Henderson, Z. [ed.] 1996. In Situ Hybridization Techniques for the Brain. John Wiley \& Sons Ltd.

Herrington, C. S., and J. O. McGee. 1992. Principles and basic methodology of DNA/RNA detection by in situ hybridization. IRL Press, New York.

Higgins, S. J. S. J. E. 1998. Molecular biology of the brain / edited by S.J. Higgins. Princeton, N.J. : Published in North America by Princeton University Press.

Hughes, J., T. W. Smith, H. W. Kosterlitz, L. A. Fothergill, B. A. Morgan, and H. R. Morris. 1975. Identification of two related pentapeptides from the brain with potent opiate agonist activity. Nature 258: 577-80.

Hultman, T., S. Stahl, E. Hornes, and M. Uhlen. 1989. Direct solid phase sequencing of genomic and plasmid DNA using magnetic beads as solid support. Nucleic Acids Res 17: 4937-46.

Israeli, R. S., W. H. Miller, Jr., S. L. Su, C. T. Powell, W. R. Fair, D. S. Samadi, R. F. Huryk, A. DeBlasio, E. T. Edwards, G. J. Wise, and et al. 1994. Sensitive nested reverse transcription polymerase chain reaction detection of circulating prostatic tumor cells: comparison of prostate-specific membrane antigen and prostate-specific antigen-based assays. Cancer Res 54: 6306-10.

Jansen, R. P. 2001. mRNA localization: message on the move. Nat Rev Mol Cell Biol 2: 247-56.

Jiang, Q., A. E. Takemori, M. Sultana, P. S. Portoghese, W. D. Bowen, H. I. Mosberg, and F. Porreca. 1991. Differential antagonism of opioid delta antinociception by [D-Ala2,Leu5,Cys6]enkephalin and naltrindole 5'- 
isothiocyanate: evidence for delta receptor subtypes. J Pharmacol Exp Ther 257: 1069-75.

John, H. A., M. L. Birnstiel, and K. W. Jones. 1969. RNA-DNA hybrids at the cytological level. Nature 223: 582-7.

Jordan, B. A., S. Cvejic, and L. A. Devi. 2000. Opioids and their complicated receptor complexes. Neuropsychopharmacology 23: S5-S18.

Kakidani, H., Y. Furutani, H. Takahashi, M. Noda, Y. Morimoto, T. Hirose, M. Asai, S. Inayama, S. Nakanishi, and S. Numa. 1982. Nature 278: 423-427.

Kar, S., and R. Quirion. 1995. Neuropeptide receptors in developing and adult rat spinal cord: an in vitro quantitative autoradiography study of calcitonin generelated peptide, neurokinins, mu-opioid, galanin, somatostatin, neurotensin and vasoactive intestinal polypeptide receptors. J Comp Neurol 354: 253-81.

Keith, D. E., S. R. Murray, P. A. Zaki, P. C. Chu, D. V. Lissin, L. Kang, C. J. Evans, and M. von Zastrow. 1996. Morphine activates opioid receptors without causing their rapid internalization. J Biol Chem 271: 19021-4.

Kenakin, T. 1996. The classification of seven transmembrane receptors in recombinant expression systems. Pharmacol Rev 48: 413-63.

Kher, R., and R. Bacallao. 2001. Direct in situ reverse transcriptase-polymerase chain reaction. Am J Physiol Cell Physiol 281: C726-32.

Kieffer, B. L. 1995. Recent advances in molecular recognition and signal transduction of active peptides: receptors for opioid peptides. Cell Mol Neurobiol 15: 615-35.

Kieffer, B. L., and C. Gaveriaux-Ruff. 2002. Exploring the opioid system by gene knockout. Prog Neurobiol 66: 285-306.

Kieffer, B. L., K. Befort, C. Gaveriaux-Ruff, and C. G. Hirth. 1992. The deltaopioid receptor: isolation of a cDNA by expression cloning and pharmacological characterization. Proc Natl Acad Sci U S A 89: 12048-52.

Kivell, B. M. 2003. Opioid receptor protein expression during development of the rat brainstem, pp. 278, School of Biological Sciences. Victoria University, Wellington.

Komminoth, P., V. Adams, A. A. Long, J. Roth, P. Saremaslani, R. Flury, M. Schmid, and P. U. Heitz. 1994. Evaluation of methods for hepatitis C virus detection in archival liver biopsies. Comparison of histology, immunohistochemistry, in-situ hybridization, reverse transcriptase polymerase chain reaction (RT-PCR) and in-situ RT-PCR. Pathol Res Pract 190: 1017-25.

Konat, G. W. 1996. Generation of high efficiency ssDNA hybridization probes by linear polymerase chain reaction (LPCR). Scanning Microsc Suppl 10: 57-60.

Koob, G. F., and F. E. Bloom. 1988. Cellular and molecular mechanisms of drug dependence. Science 242: 715-23.

Kornblum, H. I., D. E. Hurlbut, and F. M. Leslie. 1987. Postnatal development of multiple opioid receptors in rat brain. Brain Res 465: 21-41.

Kosterlitz, H. W., and S. J. Paterson. 1981. Tyr-D-Ala-Gly-MePhe-NH(CH2)2OH is a selective ligand for the mu-opiate binding site. Br J Pharmacol 73: 299.

Kraczkowski, J. J., and M. Semczuk. 2000. [Sex and changes in mu-opioid receptor density under hypoxia]. Ginekol Pol 71: 927-30.

Kream, R. M., T. Kato, H. Shimonaka, J. E. Marchand, and W. H. Wurm. 1993. Substance $P$ markedly potentiates the antinociceptive effects of morphine sulfate administered at the spinal level. Proc Natl Acad Sci U S A 90: 3564-8. 
Kruger, L., S. Saporta, and L. W. Swanson. 1995. Photographic Atlas of the Rat Brain, Photographic Atlas of the Rat Brain. Cambridge University Press.

Kwiatkowski, M., M. Nilsson, and U. Landegren. 1996. Synthesis of full-length oligonucleotides: cleavage of apurinic molecules on a novel support. Nucleic Acids Res 24: 4632-8.

Kwok, S., D. E. Kellogg, N. McKinney, D. Spasic, L. Goda, C. Levenson, and J. J. Sninsky. 1990. Effects of primer-template mismatches on the polymerase chain reaction: human immunodeficiency virus type 1 model studies. Nucleic Acids Res 18: 999-1005.

Landegren, U., and M. Nilsson. 1997. Locked on target: strategies for future gene diagnostics. Ann Med 29: 585-90.

Lewis, M. E., and F. J. Baldino. 1990. Probes for in situ hybridization histochemistry. CRC Press, Boca Raton.

Lewis, M. E., W. T. Rogers, R. G. Krause, 2nd, and J. S. Schwaber. 1989. Quantitation and digital representation of in situ hybridization histochemistry. Methods Enzymol 168: 808-21.

Liu, J. Z., K. D. Lanclos, and T. H. Huisman. 1986. Synthesis of a fixed-length single-stranded DNA probe by blocking primer extension in bacteriophage M13. Gene 42: 113-7.

Lord, J. A., A. A. Waterfield, J. Hughes, and H. W. Kosterlitz. 1977. Endogenous opioid peptides: multiple agonists and receptors. Nature 267: 495-9.

Luo, J., and F. Barany. 1996. Identification of essential residues in Thermus thermophilus DNA ligase. Nucleic Acids Res 24: 3079-85.

Luo, J., D. E. Bergstrom, and F. Barany. 1996. Improving the fidelity of Thermus thermophilus DNA ligase. Nucleic Acids Res 24: 3071-8.

Mansour, A., H. Khachaturian, M. E. Lewis, H. Akil, and S. J. Watson. 1987. Autoradiographic differentiation of mu, delta, and kappa opioid receptors in the rat forebrain and midbrain. J Neurosci 7: 2445-64.

Mansour, A., C. A. Fox, S. Burke, H. Akil, and S. J. Watson. 1995. Immunohistochemical localization of the cloned mu opioid receptor in the rat CNS. Journal Of Chemical Neuroanatomy 8: 283-305.

Mansour, A., C. A. Fox, S. Burke, F. Meng, R. C. Thompson, H. Akil, and S. J. Watson. 1994. $\mathrm{Mu}$, delta, and kappa opioid receptor mRNA expression in the rat CNS: an in situ hybridization study. Journal Of Comparative Neurology 350: 412-38.

Martin, W. R., C. G. Eades, J. A. Thompson, R. E. Huppler, and P. E. Gilbert. 1976. The effects of morphine- and nalorphine- like drugs in the nondependent and morphine-dependent chronic spinal dog. J Pharmacol Exp Ther 197: 517-32.

Mattia, A., T. Vanderah, H. I. Mosberg, and F. Porreca. 1991a. Lack of antinociceptive cross-tolerance between [D-Pen2, D-Pen5]enkephalin and [DAla2]deltorphin II in mice: evidence for delta receptor subtypes. J Pharmacol Exp Ther 258: 583-7.

Mattia, A., T. Vanderah, H. I. Mosberg, J. R. Omnaas, W. D. Bowen, and F. Porreca. 1991b. Pharmacological characterization of [D-

Ala2,Leu5,Ser6]enkephalin (DALES): antinociceptive actions at the delta non-complexed-opioid receptor. Eur J Pharmacol 192: 371-5.

Mayer, D. J., and D. D. Price. 1976. Central nervous system mechanisms of analgesia. Pain 2: 379-404. 
McLeman, E. R., J. J. Warsh, L. Ang, P. P. Li, K. S. Kalasinsky, B. M. Ross, J. Tong, G. Schmunk, V. Adams, and S. J. Kish. 2000. The human nucleus accumbens is highly susceptible to $\mathrm{G}$ protein down-regulation by methamphetamine and heroin. J Neurochem 74: 2120-6.

Mee, A. P., J. Denton, J. A. Hoyland, M. Davies, and E. B. Mawer. 1997. Quantification of vitamin D receptor mRNA in tissue sections demonstrates the relative limitations of in situ-reverse transcriptase-polymerase chain reaction. J Pathol 182: 22-8.

Mestek, A., J. H. Hurley, L. S. Bye, A. D. Campbell, Y. Chen, M. Tian, J. Liu, H. Schulman, and L. Yu. 1995. The human mu opioid receptor: modulation of functional desensitization by calcium/calmodulin-dependent protein kinase and protein kinase C. J Neurosci 15: 2396-406.

Millican, D. S., and I. M. Bird. 1997. A general method for single-stranded DNA probe generation. Anal Biochem 249: 114-7.

Minami, M., K. Maekawa, K. Yabuuchi, and M. Satoh. 1995. Double in situ hybridization study on coexistence of mu-, delta- and kappa-opioid receptor mRNAs with preprotachykinin A mRNA in the rat dorsal root ganglia. Brain Res Mol Brain Res 30: 203-10.

Morin-Surun, M. P., G. Gacel, J. Champagnat, M. Denavit-Saubie, and B. P. Roques. 1984a. Pharmacological identification of delta and mu opiate receptors on bulbar respiratory neurons. Eur J Pharmacol 98: 241-7.

Morin-Surun, M. P., E. Boudinot, G. Gacel, J. Champagnat, B. P. Roques, and M. Denavit-Saubie. 1984b. Different effects of mu and delta opiate agonists on respiration. Eur J Pharmacol 98: 235-40.

Mosberg, H. I., R. Hurst, V. J. Hruby, K. Gee, H. I. Yamamura, J. J. Galligan, and T. F. Burks. 1983. Bis-penicillamine enkephalins possess highly improved specificity toward delta opioid receptors. Proc Natl Acad Sci U S A 80: 5871-4.

Mrkusich, E. M., B. M. Kivell, J. H. Miller, and D. J. Day. 2003. Abundant expression of mu opioid receptor mRNA and protein in Purkinje cells in the cerebellum of the fetal, neonatal, and adult rat, Wellington.

Mullis, K. B., and F. A. Faloona. 1987. Specific synthesis of DNA in vitro via a polymerase-catalyzed chain reaction. Methods Enzymol 155: 335-50.

Myer, S. E. 2001. Circularisable Ligation Probes: Synthesis and use for Estimating the Frequency of Recombination at the 21-Hydroxylase Locus, pp. 254, Molecular Biology. Victoria University of Wellington, Wellington, New Zealand.

Myer, S. E., and D. J. Day. 2001. Synthesis and application of circularizable ligation probes. Biotechniques 30: 584-8, 590, 592-3.

Nakanishi, S., A. Inoue, T. Kita, M. Nakamura, A. C. Y. Chang, S. N. Cohen, and S. Numa. 1979. Nature 278: 423-427.

Namba, T., Y. Sugimoto, M. Negishi, A. Irie, F. Ushikubi, A. Kakizuka, S. Ito, A. Ichikawa, and S. Narumiya. 1993. Alternative splicing of C-terminal tail of prostaglandin E receptor subtype EP3 determines G-protein specificity. Nature 365: 166-70.

Newton, C. R., and A. Graham. 1994. PCR, Introduction to Biotechniques. BIOS Scientific Publishers Limited.

Nilsson, M., H. Malmgren, M. Samiotaki, M. Kwiatkowski, B. P. Chowdhary, and U. Landegren. 1994. Padlock probes: circularizing oligonucleotides for localized DNA detection. Science 265: 2085-8. 
Nilsson, M., K. Krejci, J. Koch, M. Kwiatkowski, P. Gustavsson, and U. Landegren. 1997. Padlock probes reveal single-nucleotide differences, parent of origin and in situ distribution of centromeric sequences in human chromosomes 13 and 21. Nat Genet 16: 252-5.

Noda, M., Y. Furutani, H. Takahashi, M. Toyosato, T. Hirose, S. Inayama, S. Nakanishi, and S. Numa. 1982. Nature 295: 202-206.

North, R. A., J. T. Williams, A. Surprenant, and M. J. Christie. 1987. Mu and delta receptors belong to a family of receptors that are coupled to potassium channels. Proc Natl Acad Sci U S A 84: 5487-91.

Nuovo, G. J. 1994. PCR in situ hybridization. Methods Mol Biol 33: 223-41.

Onogi, T., M. Minami, Y. Katao, T. Nakagawa, Y. Aoki, T. Toya, S. Katsumata, and M. Satoh. 1995. DAMGO, a mu-opioid receptor selective agonist, distinguishes between mu- and delta-opioid receptors around their first extracellular loops. FEBS Lett 357: 93-7.

Palacios, I. M., and D. St Johnston. 2001. Getting the message across: the intracellular localization of mRNAs in higher eukaryotes. Annu Rev Cell Dev Biol 17: 569-614.

Pampusch, M. S., M. A. Osinski, D. R. Brown, and M. P. Murtaugh. 1998. The porcine mu opioid receptor: molecular cloning and mRNA distribution in lymphoid tissues. J Neuroimmunol 90: 192-8.

Pan, Y. X., J. Xu, L. Mahurter, E. Bolan, M. Xu, and G. W. Pasternak. 2001. Generation of the mu opioid receptor (MOR-1) protein by three new splice variants of the Oprm gene. Proc Natl Acad Sci U S A 98: 14084-9.

Pan, Y. X., J. Xu, E. Bolan, C. Abbadie, A. Chang, A. Zuckerman, G. Rossi, and G. W. Pasternak. 1999. Identification and characterization of three new alternatively spliced mu-opioid receptor isoforms. Mol Pharmacol 56: 396403.

Pasternak, G. W., and S. H. Snyder. 1975. Identification of novel high affinity opiate receptor binding in rat brain. Nature 253: 563-5.

Pasternak, G. W., and P. J. Wood. 1986. Multiple mu opiate receptors. Life Sci 38: 1889-98.

Pasternak, G. W., S. R. Childers, and S. H. Snyder. 1980a. Opiate analgesia: evidence for mediation by a subpopulation of opiate receptors. Science 208 : 514-6.

Pasternak, G. W., S. R. Childers, and S. H. Snyder. 1980b. Naloxazone, a longacting opiate antagonist: effects on analgesia in intact animals and on opiate receptor binding in vitro. J Pharmacol Exp Ther 214: 455-62.

Pert, C. B., and S. H. Snyder. 1973. Opiate receptor: demonstration in nervous tissue. Science 179: 1011-4.

Pfeiffer, M., T. Koch, H. Schroder, M. Laugsch, V. Hollt, and S. Schulz. 2002. Heterodimerization of somatostatin and opioid receptors cross-modulates phosphorylation, internalization, and desensitization. J Biol Chem 277: 19762-72.

Roche Molecular Biochemicals. 1996. Nonradioactive In Situ Hybridisation Application Manual.

Rossi, G. C., G. P. Brown, L. Leventhal, K. Yang, and G. W. Pasternak. 1996. Novel receptor mechanisms for heroin and morphine- 6 beta-glucuronide analgesia. Neurosci Lett 216: 1-4.

Satoh, M., and M. Minami. 1995. Molecular pharmacology of the opioid receptors. Pharmacol Ther 68: 343-64. 
Scharf, S. J., G. T. Horn, and H. A. Erlich. 1986. Direct cloning and sequence analysis of enzymatically amplified genomic sequences. Science 233: 1076-8.

Schnell, S. A., W. A. Staines, and M. W. Wessendorf. 1999. Reduction of lipofuscin-like autofluorescence in fluorescently labeled tissue. J Histochem Cytochem 47: 719-30.

Schulz, S., M. Schreff, T. Koch, A. Zimprich, C. Gramsch, R. Elde, and V. Hollt. 1998. Immunolocalization of two mu-opioid receptor isoforms (MOR1 and MOR1B) in the rat central nervous system. Neuroscience 82: 613-22.

Sebastian, S., B. Hoebee, M. P. Hande, U. W. Kenkare, and A. T. Natarajan. 1997. Assignment of hexokinase types $1,2,3$ (Hk1,2,3) and glucokinase (Gck) to rat chromosome band $20 \mathrm{q} 11,4 \mathrm{q} 34,17 \mathrm{q} 12$ and $14 \mathrm{q} 21$ respectively, by in situ hybridization. Cytogenet Cell Genet 77: 266-7.

Sharma, S. K., M. Nirenberg, and W. A. Klee. 1975. Morphine receptors as regulators of adenylate cyclase activity. Proc Natl Acad Sci U S A 72: 590-4.

Simon, E. J., J. M. Hiller, and I. Edelman. 1973. Stereospecific binding of the potent narcotic analgesic (3H) Etorphine to rat-brain homogenate. Proc Natl Acad Sci U S A 70: 1947-9.

Smith, J. A., F. M. Leslie, R. S. Broide, and S. E. Loughlin. 1993. Long-term changes in striatal opioid systems after 6-hydroxydopamine lesion of rat substantia nigra. Neuroscience 55: 935-51.

Sofuoglu, M., P. S. Portoghese, and A. E. Takemori. 1991. Differential antagonism of delta opioid agonists by naltrindole and its benzofuran analog (NTB) in mice: evidence for delta opioid receptor subtypes. J Pharmacol Exp Ther 257: 676-80.

Sovago, J., D. S. Dupuis, B. Gulyas, and H. Hall. 2001. An overview on functional receptor autoradiography using [35S]GTPgammaS. Brain Res Brain Res Rev 38: $149-64$.

Stein, C. [ed.] 1999. Opioids in Pain Control: Basic and Clinical Aspects. Cambridge University Press.

Sternini, C., M. Spann, B. Anton, D. E. Keith, Jr., N. W. Bunnett, M. von Zastrow, C. Evans, and N. C. Brecha. 1996. Agonist-selective endocytosis of mu opioid receptor by neurons in vivo. Proc Natl Acad Sci U S A 93: 9241-6.

Surratt, C. K., P. S. Johnson, A. Moriwaki, B. K. Seidleck, C. J. Blaschak, J. B. Wang, and G. R. Uhl. 1994. -mu opiate receptor. Charged transmembrane domain amino acids are critical for agonist recognition and intrinsic activity. J Biol Chem 269: 20548-53.

Takeda, S., L. I. Eriksson, Y. Yamamoto, H. Joensen, H. Onimaru, and S. G. Lindahl. 2001. Opioid action on respiratory neuron activity of the isolated respiratory network in newborn rats. Anesthesiology 95: 740-9.

Terenius, L. 1973. Characteristics of the "receptor" for narcotic analgesics in synaptic plasma membrane fraction from rat brain. Acta Pharmacol Toxicol (Copenh) 33: 377-84.

Tian, M., H. E. Broxmeyer, Y. Fan, Z. Lai, S. Zhang, S. Aronica, S. Cooper, R. M. Bigsby, R. Steinmetz, S. J. Engle, A. Mestek, J. D. Pollock, M. N. Lehman, H. T. Jansen, M. Ying, P. J. Stambrook, J. A. Tischfield, and L. Yu. 1997. Altered hematopoiesis, behavior, and sexual function in mu opioid receptor-deficient mice. J Exp Med 185: 1517-22.

Tong, Y., J. Chabot, S. Shen, B. F. O'Dowd, S. R. George, and R. Quirion. 2000. Ontogenic profile of the expression of the mu opioid receptor gene in the rat 
telencephalon and diencephalon: an in situ hybridization study. $\mathrm{J}$ Chem Neuroanat 18: 209-222.

Vaccarino, A. L., G. A. Olson, R. D. Olson, and A. J. Kastin. 1999. Endogenous opiates: 1998. Peptides 20: 1527-74.

van Ree, J. M., D. de Wied, A. F. Bradbury, E. C. Hulme, D. C. Smyth, and C. R. Snell. 1976. Induction of tolerance to the analgesic action of lipotropin in C-fragment. Nature 264: 792-4.

Veatch, R. M., T. K. Adler, and E. L. Way. 1964. The importance of steric configuration in certain morphine-mimetic actions of synthetic analgesics. I Pharmacol Exp Ther 145: 11-19.

von Voigtlander, P. F., R. A. Lahti, and J. H. Ludens. 1983. U-50,488: a selective and structurally novel non-Mu (kappa) opioid agonist. J Pharmacol Exp Ther 224: 7-12.

Wang, J. B., Y. Imai, C. M. Eppler, P. Gregor, C. E. Spivak, and G. R. Uhl. 1993. mu opiate receptor: cDNA cloning and expression. Proc Natl Acad Sci US A 90: 10230-4.

Weiljlard, J., and A. E. Erikson. 1942. N-Allylnormorphine. Journal of The American Chemical Society 64: 869-870.

Wittert, G., P. Hope, and D. Pyle. 1996. Tissue distribution of opioid receptor gene expression in the rat. Biochem Biophys Res Commun 218: 877-81.

Wolozin, B. L., and G. W. Pasternak. 1981. Classification of multiple morphine and enkephalin binding sites in the central nervous system. Proc Natl Acad Sci U S A 78: 6181-5.

Wu, D. Y., and R. B. Wallace. 1989. Specificity of the nick-closing activity of bacteriophage T4 DNA ligase. Gene 76: 245-54.

Wu, D. Y., L. Ugozzoli, B. K. Pal, and R. B. Wallace. 1989. Allele specific enzymatic amplification of beta-globin genomic DNA for diagnosis of sickle cell anemia. Proc Natl Acad Sci U S A 86: 2757 60.

Xia, Y., and G. G. Haddad. 1991. Ontogeny and distribution of opioid receptors in the rat brainstem. Brain Res 549: 181-93.

Yaksh, T. L. 1988. CNS mechanisms of pain and analgesia. Cancer Surv 7: 5-28.

Yoshikawa, M., K. Yokogami, K. Kitamura, T. Kobayashi, M. Kanai, T. Taniguchi, K. Akahori, M. Nakabayashi, K. Iwao, M. Hyodo, and T. Oka. 1999. Time course of changes in mu-opioid receptor mRNA levels in the periaqueductal gray of rat brain by a single or repeated injections of antisense oligodeoxynucleotides. Jpn J Pharmacol 81: 209-15.

You, Z. B., T. M. Tzschentke, E. Brodin, and R. A. Wise. 1998. Electrical stimulation of the prefrontal cortex increases cholecystokinin, glutamate, and dopamine release in the nucleus accumbens: an in vivo microdialysis study in freely moving rats. J Neurosci 18: 6492-500.

Zadina, J. E., L. Hackler, L. J. Ge, and A. J. Kastin. 1997. A potent and selective endogenous agonist for the mu-opiate receptor [see comments]. Nature 386: 499-502.

Zagon, I. S., D. M. Gibo, and P. J. McLaughlin. 1991. Zeta (zeta), a growthrelated opioid receptor in developing rat cerebellum: identification and characterization. Brain Res 551: 28-35.

Zangen, A., and U. Shalev. 2003. Nucleus accumbens beta-endorphin levels are not elevated by brain stimulation reward but do increase with extinction. Eur J Neurosci 17: 1067-72. 
Zhang, D. Y., M. Brandwein, T. C. Hsuih, and H. Li. 1998a. Amplification of target-specific, ligation-dependent circular probe. Gene 211:277-85.

Zhang, J., G. T. Gibney, P. Zhao, and Y. Xia. 2002. Neuroprotective role of deltaopioid receptors in cortical neurons. Am J Physiol Cell Physiol 282: C122534.

Zhang, J., S. S. Ferguson, L. S. Barak, S. R. Bodduluri, S. A. Laporte, P. Y. Law, and M. G. Caron. 1998b. Role for G protein-coupled receptor kinase in agonist-specific regulation of mu-opioid receptor responsiveness. Proc Natl Acad Sci U S A 95: 7157-62.

Zimprich, A., T. Simon, and V. Hollt. 1995. Cloning and expression of an isoform of the rat mu opioid receptor (rMOR1B) which differs in agonist induced desensitization from rMOR1. FEBS Lett 359: 142-6.

Zukin, R. S., M. Eghbali, D. Olive, E. M. Unterwald, and A. Tempel. 1988. Characterization and visualization of rat and guinea pig brain kappa opioid receptors: evidence for kappa 1 and kappa 2 opioid receptors. Proc Natl Acad Sci U S A 85: 4061-5. 